Hybrid Systems

Modeling

Manufacturing and

Front Dynamics 



\title{
Hybrid Systems Modeling Manufacturing and Front Dynamics
}

\author{
Dissertation \\ zur Erlangung des Doktorgrades \\ der Mathematisch-Naturwissenschaftlichen Fakultäten \\ der Georg August Universität zu Göttingen
}

vorgelegt von

Karsten Peters

aus Rostock

Göttingen 2003 
D7

Referent:

Prof. Dr. Ulrich Parlitz

Koreferent:

Prof. Dr. Andreas Tilgner

Tag der mündlichen Prüfung: 17. Juli 2003 


\section{Kurzfassung}

Die vorliegende Arbeit befasst sich mit der Dynamik einer Klasse hybrider dynamischer Systeme, die als switched tank systems bezeichnet werden. Die Anwendung solcher hybriden Modelle auf Produktionssysteme und die Frontdynamik in Halbleiterübergittern führt zu neuen Erkenntnissen über die Natur des Zeitverhaltens dieser Systeme.

Hybride dynamische Systeme entstehen aus der Interaktion kontinuierlicher dynamischer Systeme mit Automaten. Ihr Verhalten wird daher nur in einem Zustandsraum verständlich, der sowohl kontinuierliche als auch diskrete (symbolische) Variablen enthält.

Im ersten Teil der Arbeit werden die Hintergründe der Modellierung und Untersuchung hybrider Systeme als dynamischer Systeme am Beispiel der switched tank systems dargestellt.

Nach einer kurzen Einleitung wird daher im zweiten Kapitel der Begriff des hybriden dynamischen Systems und die damit verbundene Terminologie eingeführt.

Das dritte Kapitel befasst sich mit der Klasse der Grenzkollisionsbifurkationen (border collision bifurcations), die in kontinuierlichen dynamischen Systemen nicht auftreten. Diese Bifurkationen bestimmen die Dynamik der in dieser Arbeit betrachteten Systeme.

Das vierte Kapitel untersucht die Dynamik von switched arrival und switched server systems, sowie weiterer, von diesen beiden Grundtypen abgeleiteter switched tank systems. Die Verwendung von Poincaré-Abbildungen zur Analyse der Dynamik von Hybridsystemen wird erläutert und die enge Beziehung zwischen den zwei Grundtypen herausgearbeited. Es wird gezeigt, dass diese hybriden Systeme bei Parameterveränderungen eine Vielzahl von Bifurkationen und unterschiedliche Dynamiken durchlaufen.

Der zweite Teil der Arbeit widmet sich zwei Anwendungen aus verschiedenen Gebieten der Wissenschaft.

Die Modellierung von Produktionssystemen durch hybride dynamische Systeme wird im Kapitel fünf dargestellt. Nach einer allgemeinen Disskussion des Ansatzes wird ein Modell eines Produktionssystems untersucht, das Rüstzeiten einbezieht. Mit diesem Modell und den Ergebnissen aus dem vierten Kapitel wird die logistische Leistungsfähigkeit von Produktionssystemen in verschiedenen dynamischen Regimes bestimmt. 
Es zeigt sich, dass die Leistung wesentlich durch die Dynamik beeinflusst wird, und dass in bestimmten Konfigurationen z.B. zu kleine Pufferkapazitäten zu chaotischem Verhalten führen, das mit empfindlichen Leistungsverlusten einhergeht.

Im sechsten Kapitel wird der raum-zeitliche Musterbildungsprozess in Halbleiterübergittern betrachted. Aus einer kurzen Einführung in das Übergittersystem werden wesentliche Eigenschaften des physikalischen Systems extrahiert und zur Formulierung eines hybriden Modells benutzt. Dieses Modell kann in bestimmten Parameterbereichen mit Hilfe einer eindimensionalen Abbildung analysiert werden. Das Kapitel endet mit einem Vergleich numerischer Resultate aus dem Hybridmodell und aus einer vollständigen mikroskopischen Simulation des Halbleiterübergitters, die eine bemerkenswerte Übereinstimmungen aufweisen.

Im letzten Kapitel wird ein Überblick über die Ergebnisse der Arbeit gegeben, die nicht nur die Untersuchung hybrider Systeme als dynamische Systeme zum Ziel hat, sondern durch die Anwendung solcher Modelle in zwei sehr verschiedenen Gebieten der Wissenschaft (Produktionsingenieurwesen und Halbleiterphysik) auch die potentielle Breite der Anwendung hybrider dynamischer Systeme zeigt. Wir hoffen, dass diese Beispiele die zukünftige Forschung auf dem Gebiet der Dynamik hybrider Systeme stimulieren werden. 


\section{Contents}

1 Introduction 1

$\begin{array}{lll}2 & \text { Hybrid Systems } & \mathbf{7}\end{array}$

2.1 Hybrid Dynamical Systems . . . . . . . . . . . . . . . . 7

2.1 .1 Occurrence of Hybrid Systems . . . . . . . . . . . . 8

2.1.2 Research on Hybrid Systems . . . . . . . . . . . . . 10

2.2 Notions of Hybrid Systems . . . . . . . . . . . . . . . . . . 11

2.2.1 Approach to a Definition of Hybrid Systems . . . . . . . . . . 11

2.2.2 Graph Representation . . . . . . . . . . . . . . . . . 12

2.2.3 Hybrid Trajectories . . . . . . . . . . . . . . . . . 13

2.2.4 Dynamical Properties of Hybrid Systems . . . . . . . . . . . . 15

2.3 Numerical Simulation of Hybrid Systems . . . . . . . . . . . . . . 15

2.4 Summary . . . . . . . . . . . . . . . . 16

3 Border Collision Bifurcations 17

3.1 Bifurcations of Piecewise Smooth Maps . . . . . . . . . . . . . . . . 17

3.1 .1 Preliminaries . . . . . . . . . . . . . . 18

3.2 Bifurcations of Continuous Piecewise Linear Maps . . . . . . . . . 20

3.2.1 Border Collision Pair Bifurcations . . . . . . . . . . . 21

3.2.2 Border Crossing Bifurcations . . . . . . . . . . . . 23

3.2.3 Border Collision Bifurcations in Continuous Two Dimensional Maps . . . . . . . . . . . . . . 27

3.3 Bifurcations of Piecewise Linear Maps with a Discontinuity . . . . 30

3.3.1 General Properties of Periodic Orbits . . . . . . . . . . 30

3.3.2 Period Adding Scenarios . . . . . . . . . . . . . . 32

3.3 .3 Period Increment Scenarios _. . . . . . . . . . . . . 34

3.3.4 Discontinuous Maps with Slopes Larger than One . . . . . . . 36

3.3.5 Positive Jump at the Discontinuity . . . . . . . . . . . . . . . . 39

3.4 Summary . . . . . . . . . . . . . . . . . . 39

4 Switched Tank Hybrid Systems 4

4.1 Switching Between Tanks . . . . . . . . . . . . . . . . . . . 41 
4.2 Switched Arrival and Switched Server Systems . . . . . . . . . . . 42

4.2.1 Strange Billiards and their Poincaré Maps . . . . . . . . . . . 45

4.2 .2 Limiting Cases . . . . . . . . . . . . . . . . . . 45

4.3 Dynamics and Bifurcations . . . . . . . . . . . . . . . 47

4.3.1 Properties of Poincaré Maps . . . . . . . . . . . . . . . 47

4.3.2 Bifurcations in three tank switched server systems . . . . . 50

4.3.3 Dynamics of Switching Times . . . . . . . . . . . . . . 53

4.3.4 Complexity of Symbolic Dynamics . . . . . . . . . . . . . 55

4.4 Further Models . . . . . . . . . . . . . . . . . . . . . 55

4.4.1 Modified Switching Rules . . . . . . . . . . . . . . 55

4.4 .2 An Asymmetric Billiard . . . . . . . . . . . . . 57

4.4.3 A Three Tank System with Four Discrete States . . . . . . . . 60

4.5 Summary . . . . . . . . . . . . . . . . . . 65

5 Modeling of Manufacturing Systems $\quad 67$

5.1 Manufacturing Systems and Hybrid Models . . . . . . . . . . . . . 67

5.1.1 A Brief History of Hybrid Modeling for Manufacturing Systems 69

5.1.2 Switched Arrival and Server Systems in Manufacturing . . . . 70

5.2 Manufacturing Systems with Set-up Times . . . . . . . . . . . . . . 72

5.2.1 A Model for Switched Server Systems with Set-up Time . . . 72

5.2.2 Poincaré Maps and Dynamics . . . . . . . . . . . . . . 75

5.3 Performance of Manufacturing Systems . . . . . . . . . . . . 77

5.3.1 Measures of Logistic Performance . . . . . . . . . . . . . . 77

5.3.2 The Costs of Switchings . . . . . . . . . . . . . . . 78

5.3.3 Throughput Times and their Distribution . . . . . . . 80

5.3.4 Optimization and Chaos Control . . . . . . . . . . . . . 84

5.4 Switched Discrete Deterministic Systems . . . . . . . . . . . . 85

5.4 Dynamics .................... 86

5.4.2 Small Stochastic Disturbances . . . . . . . . . . . 87

5.5 Summary . . . . . . . . . . . . . . . . . 87

6 Modeling of Front Dynamics in Semiconductor Devices 89

6.1 Fronts and Pattern Formation in Semiconductor Superlattices and Other

Spatio-Temporal Systems . . . . . . . . . . . . . . . . . 89

6.1.1 Benefits of a Generic Model . . . . . . . . . . . . . . . . 92

6.2 Modeling Front Dynamics . . . . . . . . . . . . . . . . . 93

6.3 Dynamics for Long Superlattices . . . . . . . . . . . . . . . . 97

6.3.1 Limiting Case of a Vanishing Threshold . . . . . . . . . . . . . . . 97

6.3 .2 The Parameter $p_{h} \ldots \ldots \ldots . \ldots . \ldots 99$

6.3.3 Dynamics for Three Domains . . . . . . . . . . . . . . 99

6.3.4 Obtaining Front Positions . . . . . . . . . . . . . 102 
6.3.5 Four and More Domains . . . . . . . . . . . . . . 103

6.4 Dynamics if Domains Can Traverse the Sample . . . . . . . . . . . 105

6.5 Comparison of Numerical Results . . . . . . . . . . . . . . . 107

6.6 Conclusion . . . . . . . . . . . . . . . . . . 109

7 Conclusions and Perspectives $\quad 111$

$\begin{array}{lll}\text { A Tables of Poincaré Maps } & 115\end{array}$

$\begin{array}{ll}\text { Bibliography } & 119\end{array}$ 



\section{Introduction}

This introduction provides a brief discussion of the main ideas presented in this thesis. It is outlined that this work, that might look like a collection of treatises on diverse themes basically is an investigation of one topic: The dynamics and applications of hybrid dynamical systems. Furthermore, the motivation, the objective and the structure of this thesis are clarified.

The investigation of systems that show a complex evolution in time is an important topic of modern sciences. One fundamental challenge therein is the development of models that are able to explain dynamical phenomena and to analyze them by mathematical means. Such models shall preserve the essential features of a real world system but idealize it in a way that allows us to understand it.

Judging by the title and the table of contents, this thesis concerns hybrid systems modeling of manufacturing systems and front dynamics in semiconductor superlattices. However, what is the meaning of front dynamics, and where is the connection between superlattices and manufacturing systems? And what are hybrid systems and which role do they play in these fields? For the reader familiar with all three notions, it might make sense to speak about the modeling of manufacturing systems by means of hybrid systems, but at least the connection to pattern formation in semiconductor systems needs an explanation.

A first general impression of the relations between this only seemingly distinct topics may be obtained from Fig.1.1. In contrast to standard models of nonlinear dynamics we consider hybrid systems. Roughly speaking, any dynamical system that involves the interaction of an automaton acting on a set of discrete symbols with an ordinary dynamical system is a hybrid system (Fig.1.1a). Up to now, the research on hybrid systems was mostly stimulated due to their importance in engineering applications. Previous works on hybrid systems have focussed on automaton aspects, general modeling frameworks and stability considerations. However not much is known about hybrid systems from the viewpoint of nonlinear dynamics. Studies of bifurcation phenomena and transitions to chaos as carried out in this thesis reveal new insights in the dynamics of this "exotic" class of dynamical systems.

Hybrid systems are found, for instance, in manufacturing (Fig.1.1b). Due to scarce 
resources the service of different material flows is switched according to logical rules - called policies - between competing tasks.

With the research on systems that involve processes of well separated time scales in natural sciences, as for instance in biophysics or pattern formation, hybrid modeling techniques are moving into these areas. Here the approximation of very fast processes by instantaneous changes is a reasonable method to obtain idealized models. If due to idealizations some conditions involving state variables occur, for instance in form of thresholds, the models become hybrid in fact. This was possibly not recognized in the past, since the notion of hybrid systems is not common among researchers from natural sciences, and hybrid phenomena may be hidden in sophisticated notations for equations of motions.

The systems investigated in the following are very basic hybrid models. We can think of them as a number of tanks and a server, switching between these tanks. Depending on the task of the server, the systems are called switched server systems if the server has to empty tanks (Fig.1.1c), or switched arrival systems (Fig.1.1d), if the server has to fill several tanks. The models are hybrid systems in continuous time, and if we consider the tank contents as variables, they have piecewise constant time derivatives (Fig.1.1g). Owing to this piecewise linearity the systems are special, simple examples of hybrid systems. The only nonlinearities in these systems are introduced due to switchings between the tanks, i.e. between a number of different possible values of these derivatives according to some logical rules. Therefore these systems contain not only aspects of nonlinear dynamical systems, but also aspects of automata. This fact will become more obvious, when we shall use state transition graphs of symbolic variables to investigate the dynamics.

Although graph representations borrowed from automata theory are helpful to understand the logical structure of hybrid systems and unavoidable in the field of hybrid maps, the dynamics itself should be investigated by methods from nonlinear dynamics. For hybrid systems in which switchings are triggered if the continuous state trajectory reaches certain thresholds (Fig.1.1g) the Poincaré mapping technique provides piecewise linear maps. To understand the bifurcations of the investigated hybrid systems therefore an understanding of the bifurcations of piecewise linear maps (Fig.1.1f) is fundamental. The bifurcations of piecewise linear maps are very distinct from bifurcations of smooth maps. They constitute the rich variety of border collision bifurcations. Since these border collision bifurcations are not common, we have included a chapter that provides the basic knowledge in form of an overview of border collision bifurcations of one dimensional maps.

It turns out, that despite of the remarkably simple construction of tank-switching hybrid systems they are able to show different types of bifurcations and chaotic behavior. We study the dynamics in some detail. By introducing additional switching thresholds 


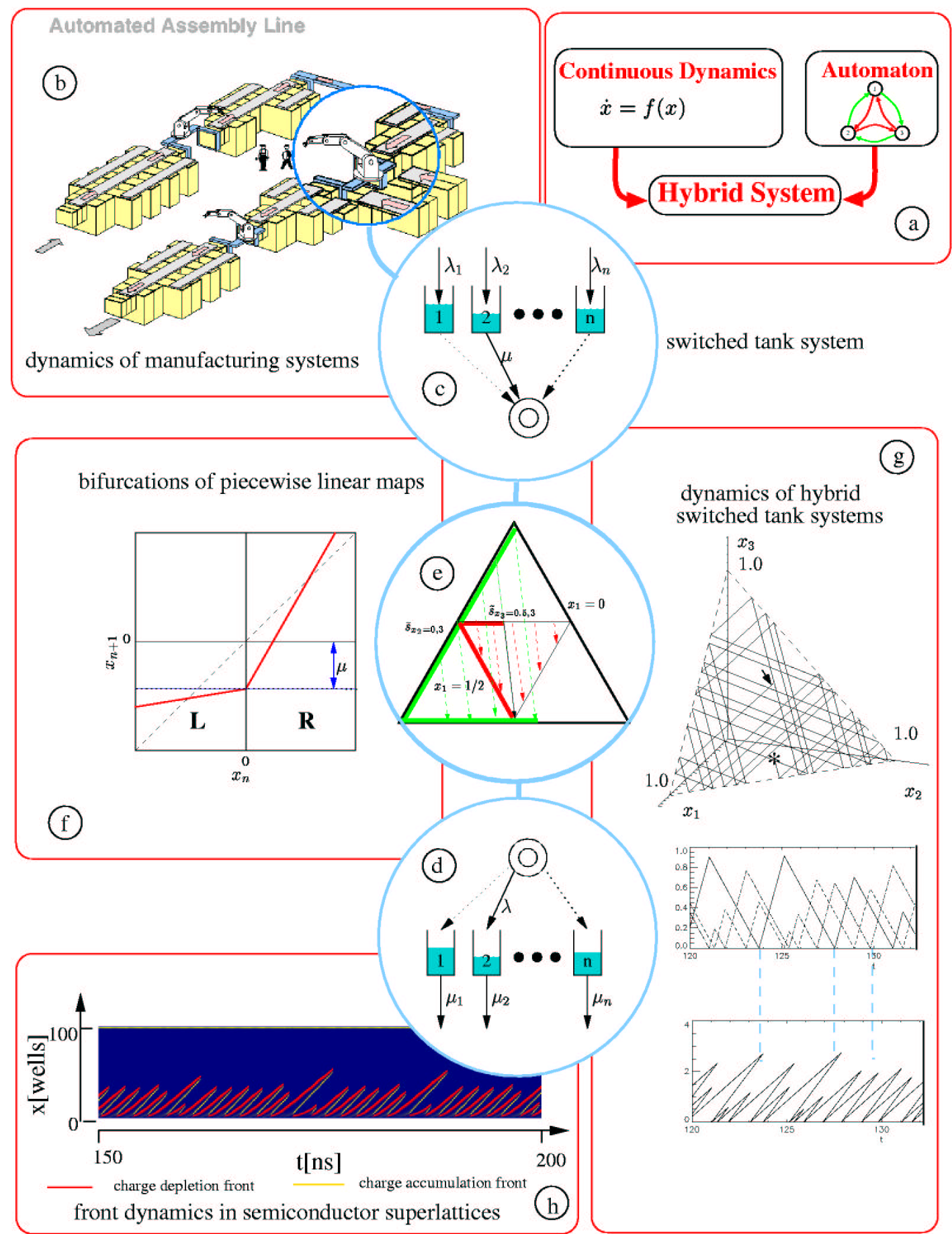

Figure 1.1: Graphical outline of this thesis containing the main ideas and the relations between the main topics in a general view. The icons surrounded by rectangular boxes stand for chapters of this work, whereas the encircled symbols give the connecting ideas. The small encircled labels are referred to in the text. 
we show in particular, that the dynamics of switched arrival systems and switched server systems occur as limiting cases in the same system. Inbetween these limiting cases characterized by periodic and chaotic behavior, a rich variety of dynamics is found, due to bifurcation scenarios built exclusively from various border collision bifurcations. Although the dynamics of the limiting cases in principle was known before, their bifurcations as well as the close relation between switched server and switched arrival systems presented in this thesis have not been discussed previously.

The first part of this thesis covers basic themes like the nature of bifurcations in switched tank hybrid models on a more general level. In the second part of this thesis the range of possible applications of the models introduced and investigated in the foregoing part is demonstrated by studying two applications from very distinct areas in detail.

One example is the investigation of basic layout structures in manufacturing systems for its dynamics dependent performance (Fig.1.1b). This study provides new insights in the importance of dimensioning buffer capacities in production planning. Therewith effects can be understood that are common on a practical level among production engineers. But in the existing frameworks of operations research (e.g. queuing theory) such dynamical effects are hardly to observe or rather difficult to explain.

The second example is located in the area of modern solid state physics. In semiconductor superlattices recently dynamical regimes were found, in which fronts of opposite charge may travel with different velocities through the lattice, annihilate one another and form complex spatio temporal patterns (Fig.1.1h). The traveling of fronts is characterized by piecewise constant velocities and very fast changes, if fronts annihilate. We derive a simple model for this pattern formation process in a bistable system under an integral over space conservation condition. With this model that belongs to the class of switched arrival systems (Fig.1.1d) not only the basic bifurcation behavior can be understood and modeled computationally effectively, but also in certain parameter ranges analyzed using a one dimensional iterated map. The traditional way of modeling these systems involves the numerical integration of typically one hundred coupled differential equations with well over 15 physically relevant parameters. In view of these facts the traditional modeling does not allow any investigation of bifurcations by analytical means.

We shall point out the surprising fact that in both applications essentially the same hybrid model is applicable. In our view this supports the conjecture that the basic hybrid systems considered here are a generic class of dynamical systems which can be widely used and may improve the understanding of complex systems in physics and other sciences.

This thesis is organized as follows. In Chap. 2 we introduce the term hybrid system and some notions that are needed in the following chapters. Also a brief review of con- 
cepts for hybrid systems that are developed in the communities of control engineers and computer scientists is given. The more formal definition, explained as reference in this chapter, will be relaxed in the main part of this work in favor of readability. In Chap. 3 the class of border collision bifurcations is presented in detail. The normal forms for this class of bifurcations are piecewise linear maps. This chapter provides the knowledge on the bifurcations that are obtained in the hybrid systems which are the main topic of this thesis.

Chapter 4 deals with the dynamics and bifurcations of switched server systems and switched arrival systems. We explain the use of Poincaré maps for the investigation of dynamical properties of hybrid systems and consider their dynamics. The hybrid modeling of manufacturing systems is discussed in Chap.5. A general discussion of the hybrid modeling approach is followed by the investigation of a model that includes set-up times in the switched server and switched arrival systems. With this model and the knowledge obtained in Chap. 4 we observe measures of logistical performance in different dynamical regimes. It turns out that the performance of manufacturing systems can significantly be affected by different dynamical behaviors.

The spatio-temporal pattern formation process in semiconductor superlattices is addressed in Chap.6. From a brief introduction into the physical system the essential features are extracted and used to formulate at first a hybrid model for front positions that can be transformed into a switched arrival hybrid system. From the latter model a flat bottom tent map is derived that captures the basic dynamical features of the system. The chapter closes with a comparison of numerical results that are obtained from the derived hybrid model and a full microscopic simulation of the semiconductor superlattice.

In the last chapter (Chap.7) we give a summary of the main results of this thesis. Since this thesis is rather a humble beginning of research in the exiting field of dynamics of hybrid systems than the contribution of a new aspect in a well covered field, we conclude with some suggestions for future research. 



\title{
2 Hybrid Systems
}

\begin{abstract}
The topic of this thesis are dynamical systems, that do not fit into the common categories of dynamical systems. We consider systems which contain two distinct components: subsystems with continuous dynamics and subsystems that act on a finite set of symbols, interacting with each other. Such systems are known as hybrid dynamical systems.

This chapter provides some fundamentals of hybrid systems. Since the notion of hybrid systems is not common the term will be clarified and illustrated with some examples (Sec. 2.1). Then a more formal framework of hybrid systems is given Sec.2.2 and some results of recent hybrid system's research will be discussed. The numerical simulation of hybrid systems as used in this work is briefly explained in Sec.2.3.
\end{abstract}

\subsection{Hybrid Dynamical Systems}

To introduce the notion of a hybrid dynamical system as it is used throughout this thesis, we shall at first recall two well known concepts, namely dynamical systems and automata.

Many textbooks on dynamical systems start with the statement that systems are dynamical if their state evolves in time and that two main types of dynamical systems can be distinguished. Systems with discrete time $\Gamma=\{t: t \in \mathbb{Z}, \mathbb{N}\}$, represented by iterated maps

$$
x_{t+1}=f\left(x_{t}\right)
$$

and dynamical systems with continuous time $\Gamma=\{t: t \in \mathbb{R}\}$, represented by differential equations

$$
\dot{x}=X(x) \text {. }
$$

In both cases $x$ is considered to be the system's continuously valued state in a state space which is a smooth manifold. More formally a dynamical system is a tuple $(\phi, M, \Gamma)$, where $\phi$ is a flow, $\mathbf{M}$ is a smooth manifold and $\Gamma$ the set of times. Throughout this thesis we assign the terms "continuous (part of) dynamical system" and "continuous (part of) state-space" to systems and parts of systems, respectively, whose 
behavior is described by Eq.(2.1) or Eq.(2.2).

On the other hand systems are known that operate on a discrete set of state variables $Q: q \in Q$, which is countable and typically finite. These systems are considered in the framework of automata theory (for an introduction refer [58]) or $^{1}$ discrete event systems [86]. Such discrete event systems usually exhibit a directed evolution of states in a logical time governed by discrete events (inputs) from a set $\Sigma$, where $e \in \Sigma$ is an event label. Their "dynamics" is given by a transition function $\delta: \Sigma \times Q \mapsto Q$ defining the jump like transitions between discrete states. Systems, or part of systems that obey the above characteristics are referred to as "discrete part of hybrid dynamical systems" in the following.

Thus the terms continuous and discrete are used here with respect to the range of state variables and not with respect to time.

Notwithstanding the great efforts of continuous dynamical system's theory and the beauty of the automata theory an impressively large group of dynamical systems and models do not fit into one of the categories since they contain aspects of both types.

Roughly speaking, any system that evolves in time and contains interactions between continuous processes and discrete automata can be seen as hybrid dynamical system usually referred to as hybrid system. Due to the mixed nature of hybrid systems their complete state space contains both continuous $\left(x \in \mathbb{R}^{n}\right)$ and discrete (symbolic) variables, and the dynamics can be represented and analyzed only with respect to this full state space.

\subsubsection{Occurrence of Hybrid Systems}

Hybrid systems arise if dynamical systems consisting of piecewise defined continuous time evolution processes are interfaced with some logical or decision making process. Such models are used in several disciplines of science and occur regularly in the modeling of technical systems.

A common example for such a hybrid system are two water tanks (see Fig.2.1a) with a water volume of $x_{i}(t)$ in each. The water flows out of the tanks with a constant rate $\mu_{i}$, and there is a switching fill unit dedicated exclusively to one tank at a time, filling this tank with a constant rate $\lambda_{i}$. Whenever a tank becomes empty $\left(x_{i}=0\right)$ The filling unit switches to this tank to refill it. If the filling unit is attached to tank $i$ we can write the equation of motion for $x_{i}$ as $\dot{x}=e_{i} \lambda-\mu$ where $e_{i}$ is the i'th canonical unit vector in $\mathbb{R}^{2}$ and $\lambda=(\lambda, \lambda)^{T}, \mu=\left(\mu_{1}, \mu_{2}\right)^{T}$ are the vectors of in and outflow rates.

${ }^{1}$ As typical in science, researchers from different fields developed distinct frameworks, methods and definitions for similar problems. Here the automata theory represents a more rigorous mathematical approach founded on the works of Turing and von Neumann, whereas the notion of discrete event systems was developed in the framework of computer sciences and control theory. 
a)

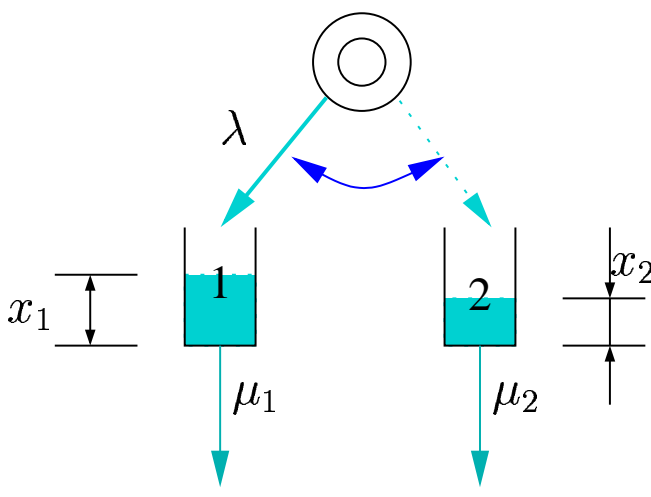

b)

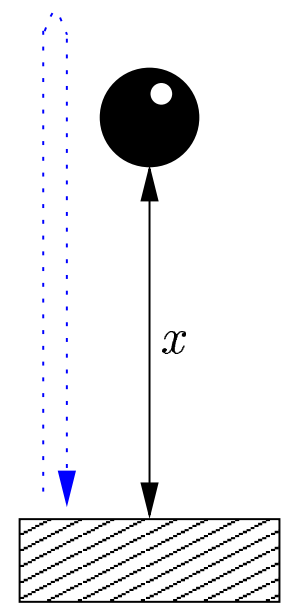

Figure 2.1: Scheme of the two watertank system (a) and the bouncing ball (b). Two standard examples for hybrid dynamical systems.

Also dynamical systems can be considered as hybrid, if jump like transitions in the continuous part of the state space occur due to collisions with obstacles for instance. A standard example for this type of hybrid systems is the vertically bouncing ball (Fig.2.1b). In its free flight state the ball moves within the gravity field ( $\ddot{x}=-g$ ). The discrete event transition takes place if the ball touches the ground. If $x=0$ the ball is reflected and a part of its energy is dissipated: $\dot{x} \mapsto-c \dot{x}$ where $0<c<1$ is a constant.

The main common feature of all hybrid systems are instantaneous jumps of either the continuous state or the "vector field", i.e. either the right or left hand side of the equation of motion written in the form of Eq.(2.2). In the hybrid system's literature $[17,18,71,97]$ four main types of hybrid phenomena are considered:

- autonomous switching: a vector field changes, if the continuous state hits certain boundaries

- autonomous impulses: the continuous state jumps discontinuously if it reaches a certain boundary

- controlled switching: the the vector field changes abruptly due to an "external" control signal

- controlled impulses: the continuous state jumps discontinuously due to an "external" control signal

A distinction similar to the notion of autonomous/controlled changes is the consideration of timed events, where the moment of transition is known a priori and state 
events where a transition occurs, if some condition involving the state variables is fulfilled.

Since an external controller or a clock principally can be included in the description of the system the distinction of autonomous and controlled hybrid phenomena is blurred, but sometimes helpful in applications. Even mixed forms of state and time events can occur, if, for instance one transition is a state event, but the following transition takes place after a certain time interval (e.g. Sec.5.2). In the same sense a mixed occurrence of switching and impulse like transitions in the same system is possible (Sec.6.4).

For technical systems it is often obvious how discrete jumps or switches influence the systems dynamics. For models of natural processes however, it depends often on the level of idealization whether a process is regarded as continuous process at fast time scales or as switching process. Sometimes new insights may become possible through idealizations that lead to hybrid models even in natural systems (the model, derived in Chap. 6 may serve as an example). Otherwise in natural sciences the hybrid nature of models may be hidden in the equations of motion in form of nonsmooth intrinsic functions, such as min, max or if statements [97] .

\subsubsection{Research on Hybrid Systems}

There has been significant research activity in the area of hybrid systems ${ }^{2}$ in the last decade involving scientist from very different fields like dynamical systems, control theory and computer science. More and more dynamical systems, that have hybrid characteristics are investigated for their dynamical behavior, sometimes without mentioning the hybrid nature of the system explicitly. Research on dynamical behavior of hybrid systems includes technical systems like mechanical systems with constraints occuring in robotics [70, 75], automotive engine control, and a large number of other intelligent control systems with a high degree of autonomy (references for such technical systems are found in the engineering literature on hybrid systems $[20,57,15,94,73])$. Furthermore a number of electronic oscillators that fall in the class of modulated relaxation oscillators may be seen as hybrid systems. Even the switching circuits, used in power electronics are hybrid.

In the modeling of biological systems hybrid models were proposed for metabolic cell regulatory networks (see [2, 56] for an introduction), transcription of genes by RNA polymerase, cell growth and division, and successfully applied to gene expression in multicellular networks [1, 14].

However, at least the engineering orientated research is not mainly interested in the dynamics of hybrid systems, but in design and verification of systems and controlers, that guarantee a stable (periodic) operation of technical devices. In this context the controllability and reachability analysis of hybrid systems plays a major rule. The

\footnotetext{
${ }^{2}$ The novelty of the subject leads to a number of different synonyms used to name hybrid systems or some special classes of this type of dynamical systems.
} 
proposed methods for this analysis are of numerical nature [15]. Thus a huge number of modeling frameworks and describing languages for hybrid systems where developed, partly to transfer stability criteria, known from other systems classes, to more ore less restricted classes of hybrid systems. An other part of modeling frameworks emerged from (numerical) simulation tools and software languages for hybrid system problems. The probably best review of the different approaches is given by research articles, that consider the equivalence of several classes of modeling frameworks (see e.g. $[20,57])$.

\subsection{Notions of Hybrid Systems}

Basically a hybrid system ${ }^{3}$ is a dynamical system that describes the evolution of a set of discrete and continuous variables in time.

\subsubsection{Approach to a Definition of Hybrid Systems}

Currently, no common definition of hybrid systems is available. Hence we will use the following basic notion of a hybrid system which mainly adopt the hybrid system definition given in [94] and [73] and incorporates the definitions of [17, 18].This definition is a straightforward adaption of the hybrid system's blueprint ${ }^{4}$ and combines the definition of automata and dynamical systems in an appropriate manner:

A hybrid system is a collection $\mathbf{H}=(M, Q, \mathcal{E}, D, \mathcal{X}, G, \mathcal{R})$, where:

$M$ is a smooth manifold that contains the continuous states $x \in M$ of $\mathbf{H}$, preferably $M \subset \mathbb{R}^{n}$;

$Q$ is a countable and finite set of (discrete) states $q \in Q$ of $\mathbf{H}$;

$\mathcal{E} \subset Q \times Q$ is the collection of discrete state transitions $(\epsilon \in \mathcal{E})^{5}$;

$\mathcal{D}=\left\{D_{q}: q \in Q\right\}$ is the set of domains ${ }^{6}$ of $\mathbf{H}$, where $D_{i} \subset q \times M \forall q \in Q$

$\mathcal{X}$ is a set of flows ${ }^{7}$ on $M$ or subsets of $M$.

$\mathcal{G}=\{G(\epsilon): \epsilon \in E\}$ is the set of guards, where for each $\epsilon=\left(q_{i}, q_{j}\right) \in E, G(\epsilon) \subset$ $D\left(q_{i}\right)$;

$\mathcal{R}=\left\{R_{\epsilon}: \epsilon \in E\right\}$ is the set of resets, where for each $\epsilon=\left(q_{i}, q_{j}\right) \in E R_{\epsilon}$ is a relation between elements of $G(\epsilon)$ and Elements of $D_{q}$ such that $R_{\epsilon} \subset G(\epsilon) \times D_{q}$, usually a

\footnotetext{
${ }^{3}$ In some parts of the hybrid systems literature even the notion hybrid automaton is common.

${ }^{4} \mathrm{~A}$ hybrid system consist of dynamical systems and an automaton interacting with each other.

${ }^{5}$ It is also common to call $\mathcal{E}$ the collection of edges because the transitions are symbolized by edges in a graph representation of the hybrid system.

${ }^{6}$ In computer science related literature the domain is called invariant set. The infelicity of this term is that there is nothing dynamical invariant in a domain.

${ }^{7}$ For instance given by a set of vector fields such that $\mathcal{X}=\left\{X_{q}: M \times Q \rightarrow T M ;(x, q) \mapsto X_{q}(x)\right\}$ and $X_{q}$ is Lipschitz on $D_{q} \forall q \in Q$.
} 
$\operatorname{map} R\left(q_{1}, q_{2}\right): G\left(q_{i}, q_{j}\right) \rightarrow D\left(q_{j}\right)$

The complete state of $\mathbf{H}$ at a time $\mathrm{t}$ is given by $(x(t), q(t)) \in M \times Q$ and the state space is

$$
D=\bigcup_{q \in Q} D_{q}
$$

$D$ is called the total domain of $\mathbf{H}$. Although different domains lie in distinct copies of $\mathrm{M}$ we use the term continuous part of the state space for that manifold which is obtained by ignoring the discrete state of the system. Formally this is done by identifying $M \times Q$ with $M$ via the diffeomorphism $(x, q) \mapsto x$. Further hybrid systems, in which the set of flows is given by a set of diffeomorphisms (i.e. a number of iterated maps) are called hybrid maps in the following.

The basic idea behind the above formalism is that the system, given by $\mathbf{H}$ evolves from a starting point $\left(\mathrm{x}_{\mathrm{x}} \mathrm{q}_{i}\right)$ insomedomain $\left(\mathrm{D}_{q i}\right)$ according to $\dot{x}=X_{q i}(x)$ until a guard $G\left(q_{i}, q_{j}\right)$ is reached, where an instantaneous switch via the reset $R\left(q_{i}, q_{j}\right)$ is made which sets the discrete state to $q_{j}$ and the continuous state to some value x'. After this transition the system evolves inside $D\left(q_{j}\right)$ up to the moment the next guard is reached.

For the water tank example (see Sec.2.1.1) we obtain with the above given formalism:

$$
\begin{aligned}
& M=\mathbb{R}^{2} \\
& Q=\left\{q_{1}, q_{2}\right\} \\
& E=\left\{\left(q_{1}, q_{2}\right),\left(q_{2}, q_{1}\right)\right\}, \\
& D\left(q_{1}\right)=\left\{q_{1}\right\} \times R^{2+}, \quad D\left(q_{2}\right)=\left\{q_{2}\right\} \times R^{2+}, \\
& X_{q_{1}, q_{2}}=e_{1,2} \lambda-\mu \\
& \left.G\left(q_{1}, q_{2}\right)=\left\{\left(q_{1}, x\right) \in D\left(q_{1}\right) \mid x_{2}=0\right\} \quad G\left(q_{2}, q_{1}\right)=\left\{q_{2}, x\right) \in D\left(q_{2}\right): x_{1}=0\right\} \\
& \left.R\left(q_{1}, q_{2}\right):\left(q_{1}, x_{1}, 0\right) \mapsto\left(q_{2}, x_{1}, 0\right), \quad R_{(} q_{2}, q_{1}\right):\left(q_{2}, 0, x_{2}\right) \mapsto\left(q_{1}, 0, x_{2}\right) .
\end{aligned}
$$

A comparison of the above set of equations with the five lines containing verbal description of the system in Sec2.1.1 may be a good argument for relaxing the notation in the remaining part of this thesis. Since the systems that are considered in the following are not much more complicated than the two tank example and no further benefits result from the formalism, we give the discrete state transitions, guards and resets in form of verbal switching rules. These rules are emphasized and numerated like equations for easy reference in the following.

\subsubsection{Graph Representation}

Since the discrete state can take only a finite number of values, it is sometimes convenient to represent a hybrid system by a directed graph (cf. Fig. 2.2). In a graph 

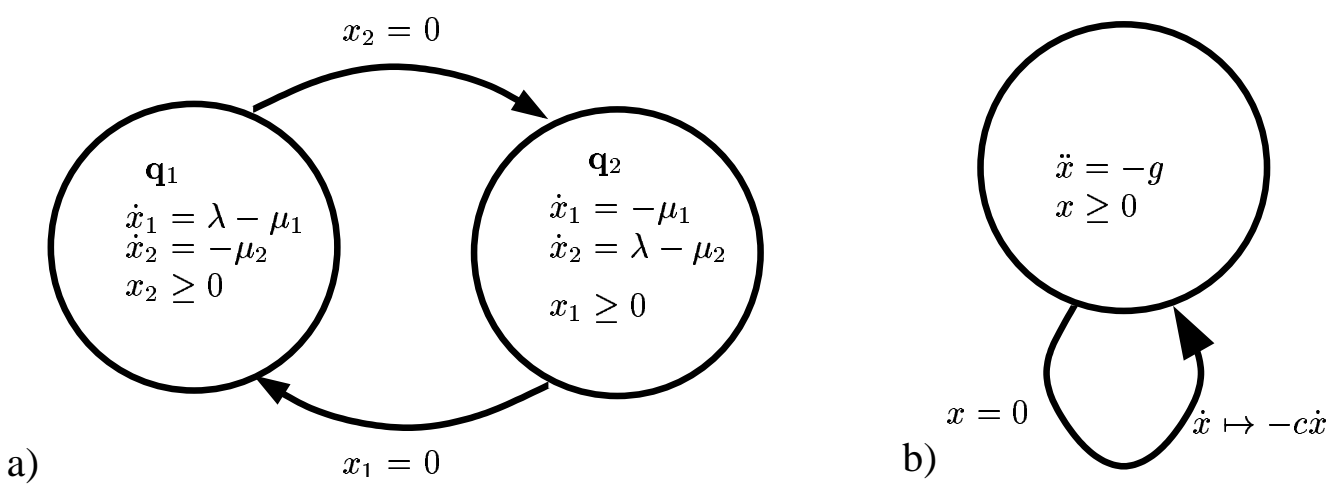

Figure 2.2: A hybrid system can be represented by a graph. a) The graph of the two watertank system. b) One of the simplest hybrid dynamical systems is a bouncing ball. The figure gives the graph representation of this hybrid system with impulse like transitions.

representation the number of nodes is equal to the number of possible discrete states.

Each node corresponds to a discrete state $q$. The notation of the vector field or equations of motion of the hybrid system in this state can be added as well as the conditions describing under which the system remains in this node. The nodes of the graphical representation are connected by directed edges, symbolizing the state transition $q_{1} \mapsto q_{2}$. Jump like changes of the continuous state of the system are noted aside the transition edges (see Fig.2.2b). In this thesis we will give graph representations of the considered systems if needed in form of state transition diagrams, where a node is only labeled with the discrete state that it represents.

A graph representation of a hybrid system emphasizes the automaton aspect of hybrid systems and clarifies its logical structure. The graph can also be taken as an alternative definition of a hybrid system $\mathbf{H}$. Furthermore, it depicts the grammar of the symbolic dynamics of the discrete states of the hybrid system (see Sec. 4.3.4).

\subsubsection{Hybrid Trajectories}

For every $(x, q) \in D(\mathbf{H})$ the action of $\mathbf{H}$ generates a trajectory of the hybrid system $\mathbf{H}$. If the flow for the continuous part of the hybrid system is given by a differential equation the trajectory is continuous inside the domains of $\mathbf{H}$, where the discrete state $q(t)=q$ remains constant. If a guard is reached at time $t_{i}$, the full state of the system changes discontinuously. We write $\left(x\left(t_{i}\right), q\left(t_{i}\right)\right) \mapsto\left(x\left(t_{i}^{+}\right), q\left(t_{i}^{+}\right)\right)$, where $f\left(t^{+}\right)$denotes the usual limit from the right of any $f$ at time $t$.

It is generally assumed that the trajectories of hybrid systems are piecewise continuous from the right, if the limit exists ${ }^{8}$. The times $t_{i}$ are called event times. The series

\footnotetext{
${ }^{8}$ Of course there is some problem, if an infinite number of switchings occur in finite time. However, the not resolved problems of a consistent mathematical framework for hybrid dynamical systems are
} 
of event times of a hybrid dynamical system is a point process.

The sequence $\tau=\left\{I_{i}\right\}_{i=0}^{N}$ of intervals $I_{i}=\left[t_{i}, t_{i}+1\right]$ is also called a (forward) $h y$ brid time trajectory. This sequence can be either infinite $(N \rightarrow \infty)$ or finite. $N(\tau)$ is the size of the hybrid time trajectory and by $\{\tau\}$ we denote the set $(1,2, \ldots, N)$ and $(1,2, \ldots)$ for $N \rightarrow \infty$, respectively. If $N(\tau)$ is finite the interval $I_{N}$ is either $I_{n}=\left[t_{N}, t_{N+1}\right]$ with $t_{i}<t_{N+1} \forall i$ or $I_{n}=\left[t_{n}, \infty\right)$.

A triple $\chi=(\tau, q, x)$ with $\tau$ being the hybrid time trajectory, $q:\{\tau\} \rightarrow Q$ a map and $x_{i}: I_{i} \rightarrow \mathrm{M}$ a collection of maps, is also called an execution of $\mathbf{H}$. Thus execution is a notion, that approaches the trajectory of hybrid systems from automaton ideas. For a precise definition refer [73]. The execution time of $\chi$ is

$$
\tau_{\infty}(\chi)=\sum_{i=0}^{N(\tau)}\left(t_{i+1}-t_{i}\right)=\lim _{i \rightarrow N(\tau)} t_{i+1}-t_{0}
$$

It is clearly a specific hybrid dynamical system's phenomenon, that there are trajectories which terminate in a finite time. Hence the following notions are used:

A execution is called infinite, if $N(\tau)=\infty$ or $\tau_{\infty}(\chi)=\infty$.

A execution is Zeno ${ }^{9}$ if $N(\tau)=\infty$ but $\tau_{\infty}<\infty$

The execution time of a Zeno execution is called a Zeno time. A (forward) trajectory which corresponds to a Zeno execution should be called Zeno trajectory. If we consider trajectories of hybrid dynamical systems we usually mean trajectories that correspond to infinite executions.

An example for Zeno trajectories is the water tank with $\lambda<\mu_{1}+\mu_{2}$. For a given total content $c=x_{1}(0)+x_{2}(0)$ at time $t_{0}$ the Zeno time is $t_{z e n o}=c /\left(\mu_{1}+\mu_{2}-\lambda\right)$. During this time the total content of the two tanks becomes smaller and smaller and the closer the system comes to zero total content the faster the switchings occur. Even the bouncing ball has only zeno trajectories since it stops if the initial total energy is completely dissipated due to an infinite series of bounces.

In hybrid systems literature often the set of reachable states of $\mathbf{H}$ is considered, which are all states that can be reached from a set of initial conditions within a finite time. Furthermore, hybrid systems where for every $(x, q) \in D$ an infinite (forward) execution exists are called non-blocking. All hybrid systems considered in this thesis are deterministic and non-blocking, i.e. for every $p \in D$ exists a unique infinite execution. Generally in a deterministic non-blocking hybrid dynamical system the resets have to be functions, the guards have to be mutually disjoint sets and whenever a trajectory is at the boundary of a domain it has to hit a guard.

not addressed here.

${ }^{9}$ The name Zeno is a reminiscence of the paradoxon about Achill and the tortoise given by the greek philosopher Zeno. As is generally known, the paradoxon is resolved by the observation that an infinite series can converge to a finite sum. Thus even in hybrid systems infinite discrete transitions can occur in finite time. 


\subsubsection{Dynamical Properties of Hybrid Systems}

From the foregoing sections it is obvious that a hybrid dynamical system in general can be a complicated (and up to now not well explored) object.

It would be desirable to achieve a theory of hybrid dynamical systems as dynamical systems in the future. Recently in this direction a number of attempts have been made concerning the transfer of stability notions from dynamical systems to hybrid systems [108]. Especially the use of piecewise defined Lyapunov functions [19] for considering Lyapunov stability is well established meanwhile [35]. A further basic approach is developed in [94] where the notion of a hybrifold was introduced. A hybrifold is an object consisting of manifolds glued together at the guard edges, which is mathematically not easy to handle because of its generally complicated topology and its partial open, partial closed boundary. Hybrifolds are in consequence similar to branched manifolds which are considered to analyze the topological structure of smooth chaotic dynamical systems. For an introduction to topological analysis of chaotic systems cf. [52]

In conclusion we state that the dynamics of hybrid systems is not well understood today.

\subsection{Numerical Simulation of Hybrid Systems}

Since discrete events are the basic features in every hybrid system, any numerical calculation must be performed on an event by event basis.

The core of the event driven simulation is the organization of an event list, which contains all known future events and their event times. Further a simulation clock is needed that holds the actual time of simulation. Given the full state of the system at time $t$ all possible future events connected with that state can be obtained for a deterministic hybrid system and have to be noted in the event list. Than the simulations proceeds as follows:

1. Pick the next event form the event list and set the simulation clock to its event time.

2. Apply, depending on the event the reset map and set the system into its next discrete state.

3. Remove all future events, that do not occur since the discrete state of $\mathbf{H}$ has changed from the event list.

4. Compute all future events that may occur now and their event times.

5. Store this future events in the event list and rearrange it according to the event times. 


\section{Return to step 1.}

Due to this structure a simulation program for hybrid systems is programmed preferably in an object orientated manner. The estimation of the next event time can be a challenging task for hybrid systems where the continuous state is given by a nonlinear differential equation. In the systems considered in this work we are not in the need of numerical integration of nonlinear differential equations and approximating the moment of guard reaching. All events are either timed, or their the time distance towards a possible future event time can be calculated directly from equations in form $\Delta t_{(m+1)}=\left(S+x\left(t_{m}\right)\right) / \lambda$ where $S$ is a threshold, $x\left(t_{m}\right)$ a continues state at the present event time $t_{m}$ and $\lambda$ any rate. In this regard the numerics is exact. Furthermore, it is fast, since only event times have to be calculated. And if values for variables inbetween two event times are needed, they can be also obtained by linear relations.

A more comprehensive guide to event driven simulations is found in $[48,65,77]$. Furthermore a number of software packages and tool boxes for the simulation of more or less restricted classes of hybrid systems especially for engineering purposes is available today.

\subsection{Summary}

Hybrid systems arise in a wide range of engineering applications as well as in models of natural processes. Sometimes the hybrid nature of models is very obvious whereas in other cases the hybrid nature of dynamical systems is hidden in discontinuities of the equations of motion.

Despite of the fact that hybrid dynamical systems have received a considerably interest in the engineering community during the last decade we are far away from a comprehensive theory of hybrid dynamical systems as it exists for continuous dynamical systems.

Nonetheless the concept of hybrid dynamical systems may be useful elsewhere for the investigation of some other models not only in technics. In the switched tank systems considered in this thesis, the hybrid modeling allows a fast and exact numerical simulation by discrete event algorithms. 


\title{
3 Border Collision Bifurcations
}

\begin{abstract}
In the following chapters of this work we are dealing with Poincare maps that consist of several linear segments. Therefore an introduction to bifurcation phenomena emerging in piecewise linear maps is given. The most simple case of one-dimensional maps consisting of two segments will be discussed in more detail. A short overview of basic notions (Sec.3.1) is followed by a review of (border collision) bifurcations of maps that are continuous, but have a discontinuous derivative. Then the bifurcation scenarios associated with a discontinuity in the map are discussed in Sec. 3.3.
\end{abstract}

\subsection{Bifurcations of Piecewise Smooth Maps}

Bifurcation generally means some branching process, in which the qualitative topological picture of an attractor alters with the change of the parameters of a dynamical system. The literature dealing with bifurcation theory (e.g. [30]) is in general focused on bifurcations of dynamical systems arising from (everywhere) differentiable processes.

For the understanding of hybrid sytems the bifurcations of piecewise smooth maps are important. Such maps arise in a very natural way from the discrete modeling of technical and natural systems [31, 28, 29, 88]. Especially the derivation of such piecewise smooth maps for systems in power electronics [80, 7, 103] has led to an increasing interest in systematic investigations of bifurcations in such maps during the last years.

Usually a bifurcation occurs if a periodic point of a map looses its stability due to a change of parameters. If a parameter is smoothly changed in a differentiable map the eigenvalue also changes in a smooth way and a local bifurcation occurs when crossing the unit circle. For piecewise smooth maps the situation is a little different. Here the eigenvalue changes abruptly if a periodic point leaves a smooth segment of the map. This leads to bifurcations that are very distinct from bifurcations of smooth maps. Since a point of discontinuity of either the map or its derivative is a border between to smooth segments of the map, the term border collision bifurcation was assigned to this type of bifurcations of piecewise smooth maps (probably the term emerged firstly in [81]). Border collision bifurcations are also called $C$-bifurcations after the earlier 
works of M.I. Feigin [49, 50, 51, 40]. The class of border collision bifurcation phenomena includes a rich variety of different bifurcations as for instance bifurcations from a fixed point attractor to a higher periodic attractor or even a chaotic one.

Hence the bifurcation parameters under consideration are unusual. Generally we consider maps where the slopes of the segments are fixed and a bifurcation parameter which tunes the position of the segments relative to the diagonal $x_{n+1}=x_{n}$. This is exactly the situation that we face with diverse Poincaré maps appearing in the following chapters.

For the following discussion we consider piecewise linear maps with two segments of different slope, which are the most simple examples among this class of iterated maps.

\subsubsection{Preliminaries}

Before we start an overview of bifurcation phenomena in piecewise linear maps let us briefly recall some basic notions by means of one dimensional maps. A concise introduction to the theory of one dimensional maps is provided by several text books (e.g. [33]).

A map $f: I \rightarrow I$ with $I \subset \mathbb{R}$ is called smooth if it has a continuous derivative. The map is piecewise smooth ${ }^{1}$ if it has a finite number of points $c_{k} \in I, k \in \mathbb{N}$ such that $f$ is smooth on $I_{k}=\left[c_{k-1}, c_{k}\right)$. A point $c_{k}$ is a turning point of $f$ if in an open neighborhood of $c_{k}$ the map is strictly increasing on one side of $c_{k}$ and strictly decreasing on the other. Maps with one turning point are unimodal maps. If $c_{k}$ is a turning point or a discontinuity of $f$, we call $c_{k}$ a critical point of $f$. The trajectory (or orbit) of a point $x_{0}$ is $f^{n}\left(x_{0}\right)_{n=0}^{\infty}$ and it is periodic of period $m$ if $m$ is the smallest number such that $f^{n+m}\left(x_{p}\right)=f^{n}\left(x_{p}\right)$. An orbit of period $m$ is typical, if the derivative of the $m$ 'th iterate of the map at the orbit's location exists and this derivative $\epsilon$ (the eigenvalue) is neither 1 nor -1 . In other words: its stability properties are ensured by the knowledge of the derivative. The orbit is called a flip saddle if $\epsilon<-1$, a regular saddle if $\epsilon>1$ and an attracting periodic point if $|\epsilon|<1$. The Lyapunov number of a trajectory is $L\left(x_{0}\right)=\lim _{n \rightarrow \infty}\left[\left|\left(f^{n}\right)^{\prime}\left(x_{0}\right)\right|\right]^{1 / n}$ if this limit exists, and the Lyapunov exponent is $\lambda=\log \left(\left|L\left(x_{0}\right)\right|\right)$. A periodic orbit is often called super stable if its Lyapunov exponent is $-\infty$.

In connection with bifurcation scenarios sometimes the question of scaling laws and corresponding scaling constants is considered. For instance all unimodal smooth maps belong to the same Feigenbaum universality class with universal Feigenbaum

\footnotetext{
${ }^{1} \mathrm{By}$ the notion piecewise smooth we do not imply that the map is continuous.
} 
constants $\alpha_{F} a n d \delta_{F}$. The scaling properties reflect the self similar structure of a bifurcation diagram. Usually two constants appear. The first one $\delta$, denotes the scaling constant in the parameter space, and the second $\alpha$, the one in state space ${ }^{2}$. The constant $\delta$ is defined by:

$$
\delta=\lim _{n \rightarrow \infty} \delta_{n}=\lim _{n \rightarrow \infty} \frac{a_{n-1}-a_{n}}{a_{n}-a_{n+1}}
$$

where $a_{n}$ is the bifurcation parameter's value at the n'th bifurcation point. The scaling constant $\alpha$ is given by ${ }^{3}$ :

$$
\alpha=\lim _{n \rightarrow \infty} \alpha_{n}=\lim _{n \rightarrow \infty} \frac{\left.\left(x_{0}-x_{1}\right)\right|_{a=a_{n}}}{\left.\left(x_{0}-x_{1}\right)\right|_{a=a_{n+1}}}
$$

where $\left.\left(x_{0}-x_{1}\right)\right|_{a=a_{n}}$ is the distance between two subsequent points of a periodic orbit at the bifurcation parameter's value $a_{n}$. In the Feigenbaum scenario the parameter $a_{n}$ for the superstable period- $2 \mathrm{n}$ orbits is used for determining $\alpha$, but if such an orbit does not exist another choice must be made. The definition of these scaling constants implies, besides the existence of the limit $n \rightarrow \infty$, a scaling that follows a power law. As we will see below such constants are not universal in the case of piecewise linear maps.

Another useful method for investigating the dynamics of one dimensional maps is the consideration of symbolic dynamics. For one dimensional maps with one critical point $c$ to each orbit a binary symbolic sequence can be assigned by labeling the touched branch according to

$$
J_{i}=\left\{\begin{array}{lll}
\mathbf{0} & \text { for } & x_{i} \leq c \\
\mathbf{1} & \text { for } & x_{i}>c
\end{array}\right.
$$

For 0,1 also the symbols $\mathrm{L}, \mathrm{R}$ will be used to denote the branches. The symbol sequence of $\left\{J_{i}\left(f^{i}\left(x_{0}\right)\right)\right\}_{i \geq 0}$ is the $f$-itinerary of $x_{0}$. The itinerary of $f(c)$ is known as the kneading sequence $\bar{K}(f)$.

Using the symbol sequence of an orbit a winding number is defined by

$$
r=\lim _{n \rightarrow \infty} \frac{\sum_{i=1}^{\infty} J_{i}}{n}
$$

It is also useful to consider for periodic orbits a reinjection number $N$, counting the number of changes from $L$ to $R$ (or $R$ to $L$ ) within the period, i.e. the number of $\{10\}$

\footnotetext{
${ }^{2}$ For the sake of completeness the values of the two famous Feigenbaum constants for maps with quadratic maxima are noted: $\alpha_{F} \approx 2.502908 \ldots$ and $\delta_{F} \approx 4.669202 \ldots$

${ }^{3} \alpha$ describes in the Feigenbaum scenario the scaling of subsequent fork openings in the period doubling cascade.
} 


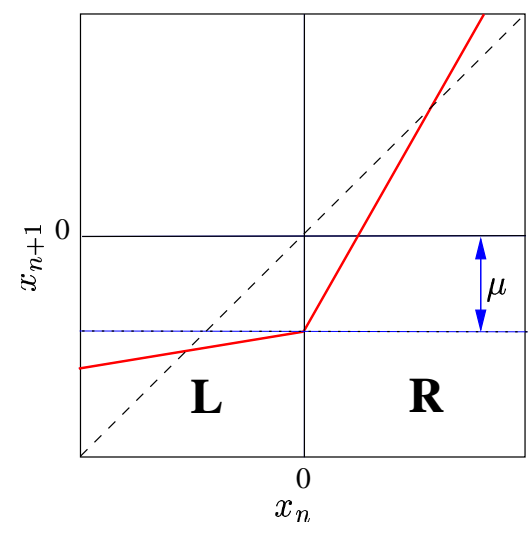

Figure 3.1: A continuous, piecewise smooth one-dimensional map. The bifurcation parameter $\mu$ is indicated. Here is $0<a_{L}<1$ and $1<a_{R}$, such that the stable fixed point in $L$ for $\mu<0$ looses its stability if $\mu$ crosses zero. This corresponds to case 1'a in Fig.3.2.

subsequences within a period ${ }^{4}$.

\subsection{Bifurcations of Continuous Piecewise Linear Maps}

If a one-dimensional piecewise smooth map is continuous but has a derivative which is not continuous at one point, this point is the "border" between two smooth segments of the map. As shown in [7] every one dimensional map with this behavior and a single critical point can be transformed into the following normal form:

$$
f(x)=\left\{\begin{array}{l}
f_{L}(x)=a_{L} x+\mu \text { for } x \leq 0 \\
f_{R}(x)=a_{R} x+\mu \text { for } x>0 .
\end{array}\right.
$$

This normal form is a piecewise linear map with three parameters $\left(a_{L}, a_{R}, \mu\right)$ and one border located at $x=0$. The slopes of the left and the right segment of the map are denoted by $a_{L}$ and $a_{R}$ respectively and are assumed to be finite ${ }^{5}$. Figure 3.1 shows an example of such a map. If we consider $\mu$ as bifurcation parameter an orbit or attractor changes its stability if a fixed point or a periodic point hits the border of a linear segment at $\mu=0$.

The nature of this bifurcation depends only on the values of $a_{L}$ and $a_{R}$ because $\mu \neq 0$ determines only the size of an orbit that exists for all $\mu<0$ and $\mu>0$,

${ }^{4}$ Both winding number and reinjection number are associated with different qualities of an orbit. Consider for instance a periodic orbit with the symbol sequence (1010) and an other with (1100). Both have a winding number $\mathrm{r}=1 / 2$ but the reinjection number of the first orbit is $N=2$ whereas the reinjection number of the second is $N=1$.

${ }^{5}$ Piecewise smooth maps with one side infinite partial derivatives arise e.g. for grazing impact oscillators $[27,34]$ and some laser systems [80]. Border collision bifurcations in maps with such singularities are also called grazing bifurcations. For a discussion see [39]. 


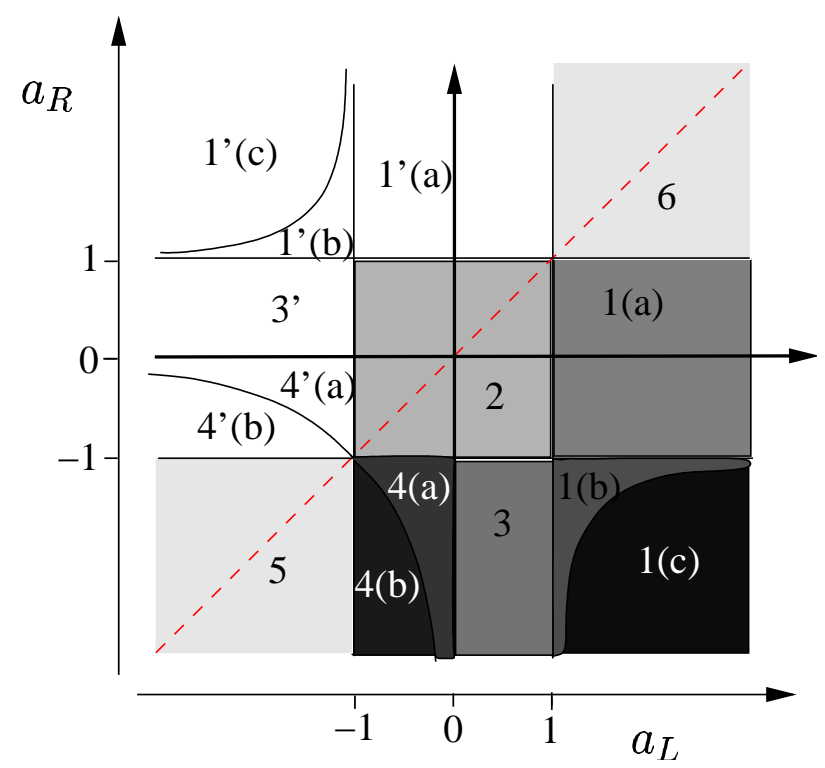

Figure 3.2: Overview of the $a_{L}-a_{R}$ parameter space of continuous piecewise linear maps with two segments. Shown are regions with the same bifurcation phenomena (cf. [8]). The labeling of the regions denotes the cases that are discussed in the text. The primed numbers show regions with the same bifurcation phenomena for an inverse border crossing due to the symmetry indicate by the dashed line.

respectively. If (and only if) the map (3.5) has a stable periodic orbit or even a chaotic attractor for $\mu=1$ then it has it for all $\mu>0$. These orbits scale linear with $\mu$. If $\{x\}$ is a trajectory for $\mu=1$ then the similar trajectory for all other $\mu$ is given by $\{\mu x\}$. The same statements apply to attractors for $-\mu>0$. The study of border collision bifurcations, in other words has to examine the attractors of (3.5) for a given $a_{L}, a_{R}$ at $\mu= \pm 1$.

Furthermore we have a symmetry in the $\left(a_{L}, a_{R}\right)$ parameter space. If a certain kind of bifurcation occurs for $a_{R}<a_{L}$ when $\mu$ is increased through zero, an analogous situation occurs if $a_{L}^{\prime}=a_{R}$ and $a_{R}^{\prime}=a_{L}$ and $\mu$ is reduced through zero. Due to this symmetry we restrict our following discussion of bifurcations of the map (3.5) to the parameter area $a_{R} \leq a_{L}$. For an overview of the below discussed cases refer Fig.3.2.

\subsubsection{Border Collision Pair Bifurcations}

If

$$
a_{L}>1 \text { and } a_{R}<1
$$

then there is no fixed point for $\mu<0$ while for $\mu>0$ two -possibly unstable- fixed points

$$
L^{*}=\mu /\left(1-a_{L}\right) \quad \text { and } \quad \mathrm{R}^{*}=\mu /\left(1-a_{R}\right)
$$




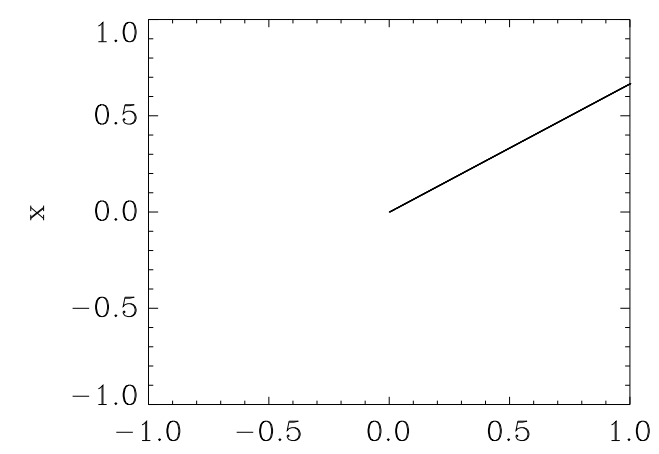

a)

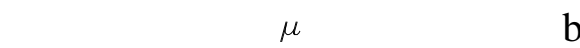

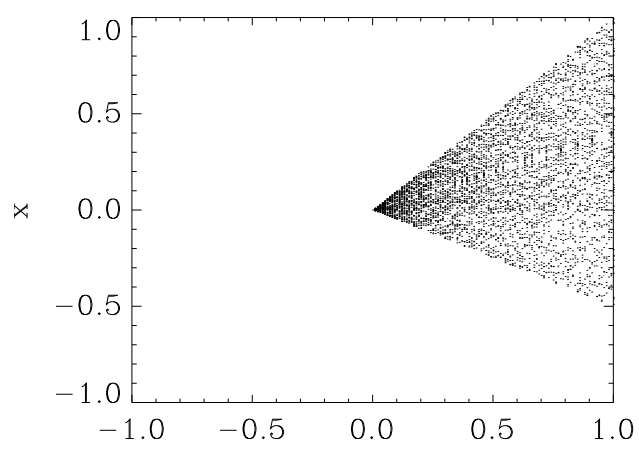

b)

Figure 3.3: Bifurcation diagrams of border collision bifurcations for the map (3.5). a) (Case 1a) with $a_{L}=-0.2, a_{R}=-0.9$ where a unique stable fixed point is born at the bifurcation point $\mu=0$. b) The border collision bifurcation (case $1 b$ ) of the normal form with $a_{L}=1.05, a_{R}=-1.2$. A chaotic attractor is born at the bifurcation point.

in $L$ and $R$ respectively, exist. Because of this emergence of fixed points at the border collision point this bifurcation was named border collision pair bifurcation.

\section{Case 1 a}

If the slope $\left|a_{R}\right|<1$ the fixed point in the right segment is stable for $\mu>0$. Furthermore it is attracting inside the interval $\left(L^{*},+\infty\right)$. Thus we obtain a bifurcation from no attractor $(\mu<0)$ to a unique stable period-1 attractor for $\mu>0$. This scenario is shown in Fig.3.3a.

\section{Case 1 b}

If $a_{L}>1$ and $a_{R}<-1$ both $L^{*}$ and $R^{*}$ are unstable. Thus for $\mu>0$ only a chaotic attractor may exist (see Fig.3.3b and Fig.3.4a for examples). If a chaotic attractor exists the chaos is robust [10].

For most smooth chaotic systems a dense set of periodic windows for any range of parameter values is obtained (for instance in the logistic map). For non-smooth systems by contrast parameter ranges are obtained, where a in neighborhood in parameter space a unique chaotic attractor without any periodic attractors in that neighborhood exists. The term robust chaos was introduced ${ }^{6}$ for this phenomenon in [10].

The parameter range for the existence of a stable chaotic attractor is bounded since no point of an attractor can be located right of $L^{*}$, where every point is mapped to $-\infty$. Therefore a stable attractor exists, as long as

$$
a_{R}>-\frac{a_{L}}{\left(a_{L}-1\right)}
$$

\footnotetext{
${ }^{6}$ It was argued in [10], that this type of chaos may be important in technical applications where a reliable operation under chaos is required (even if a slight fluctuation of parameters are unavoidable).
} 

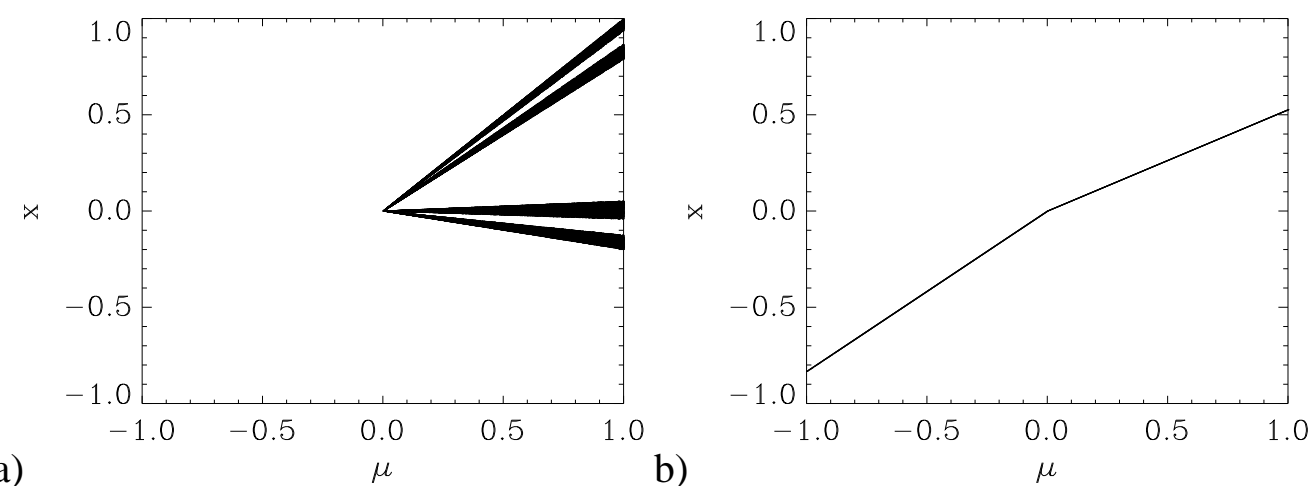

a)

b)

Figure 3.4: Bifurcation diagrams of border collision bifurcations of map (3.5). a) A special situation of case $1 b$ where a four piece chaotic attractor is born at the bifurcation point $\left(a_{L}=1.5, a_{R}=-1.2\right)$. b) An example of case $2\left(a_{L}=-0.2, a_{R}=-0.9\right)$.

Inside this region a special parameter range exists for

$$
a_{L}<\frac{a_{R}}{\left(1-a_{R}^{2}\right)} .
$$

where multiple piece chaotic attractors of skewed tent maps arise for $\mu>0$. The discussion of these possible chaotic attractors is postponed to the following section in the context of case 4 where the borders for an emergence of n-piece chaotic attractors will be reviewed.

\section{Case 1 c}

In the parameter region $a_{L}>1$ and $a_{R}<-a_{L} /\left(a_{L}-1\right)$ neither for $\mu<0$ nor for $\mu>0$ a stable attractor exists.

\subsubsection{Border Crossing Bifurcations}

In all regions of the parameter space that do not belong to the area defined in Eq.3.6, an existing fixed point crosses the border if $\mu$ is varied through zero and changes its stability properties. The border crossing of fixed points may alternatively be interpreted as the existence of a pair of fixed points where one is virtually in the sense that the branch to which it belongs does not exist where this fixed point is located. If $\mu$ crosses zero the virtuality is interchanged between the two fixed points.

\section{Case 2}

If both

$$
\left|a_{R}\right|<1 \text { and }\left|a_{L}\right|<1
$$

there is obviously a stable fixed point $L^{*}$ for $\mu<0$ and $R^{*}$ for $\mu>0$ (Eq.3.7). Thus there is a unique stable period- 1 attractor which changes its path at $\mu=0$, similar to 
a tangent bifurcation in smooth maps. If $a_{L}$ and $a_{R}$ contain an opposite algebraic sign the direction of the path will be also opposite to its former direction after the bifurcation.

\section{Case 3}

The parameter region

$$
0<a_{L}<1 \text { and } a_{R}<-1
$$

shows a variety of different border crossing bifurcations. For $\mu<1$ the map has a stable fixed point at the left branch. But for $\mu>0$ the map becomes a skewed tent map with one segment expanding and one contracting. Due to its importance and its rich dynamical behavior skewed tent maps have attained a considerable interest [74, 80, 79, 16].

The first result that was reported in [74] and [80] using results from [60] deals with the existence and stability of periodic orbits in the skewed tent map family (with $a_{L}<a_{R} /\left(1+a_{R}\right), a_{R}<0$ with respect to the symmetries and the stability result (3.8) reported above).

All stable period-n orbits are of the symbolic form $\left\{10^{n-1}\right\}$. The parameter regions where stable periodic orbits of period $n \geq 2$ appear are determined by the region which ensures the existence of an period-n orbit, given by $a_{R} \leq-\left(1-a_{L}^{n-1}\right) /\left(a_{L}^{n-2}-\right.$ $\left.a_{L}^{n-1}\right)$ and the curve, where the period- $n$ orbit looses its stability, i.e. $a_{R}>-a_{L}^{-(n-1)}$. Therewith the region where a stable period-n orbit exists is given $[74,80]$ by

$$
P_{n}\left(a_{L}, a_{R}\right):-a_{L}^{1-n}<a_{R}<a_{L}\left(1-a_{L}\right)^{-1}\left(1-a_{L}^{1-n}\right) .
$$

With $a_{L}=0.5$ we obtain for instance a period- $n$ attractor for $-2^{n-1} \geq a_{R}>$ $-\left(2^{n-1}-1\right)$. The two curves of the lower and upper boundary of this region intersect in points $O_{n}=\left(a_{L}, a_{R}\right)$, which define the endpoints of the stability regions. This endpoints are located on the $a_{R}<-1$ branch of the hyperbola $a_{R}=a_{L} /\left(1-2 a_{L}\right)$ where the first coordinate of $O_{n}$ is the root of $a_{L}^{n}-2 a_{L}+1=0$ in the interval $(0.5,1)$ [74]. At the right hand side of this hyperbola the region given by (3.10) is an open and nonempty set.

The second result on skewed tent-maps, that was found in [74, 80], is the order of attractors in the parameter regions, where no stable periodic orbits exist. If $a_{R}$ decreases through the stability boundary of $P_{n}, a_{R}=-a_{L} /\left(a_{L}-1\right)$, a 2n-piece chaotic attractor emerges for all $n>2$. This 2 n-piece interval-circle is obtained by iterating the interval $\left(f^{2 n}(\mu), \mu\right)$ under $f$, especially it contains the interval $\left(f^{n}(\mu), f^{3 n}(\mu)\right)$. If $a_{R}$ is further decreased the intervals $\left(f^{2 n}(\mu), \mu\right)$ and $\left.f^{n}(\mu), f^{3 n}(\mu)\right)$ come in contact and merge ${ }^{7}$, forming a $n$-piece chaotic attractor. The bifurcation line where this

\footnotetext{
${ }^{7}$ Band-merging bifurcations are typical bifurcations for unimodal maps and are observed also in smooth maps as for instance the logistic map. Nonetheless, in the piecewise linear map there is no
} 

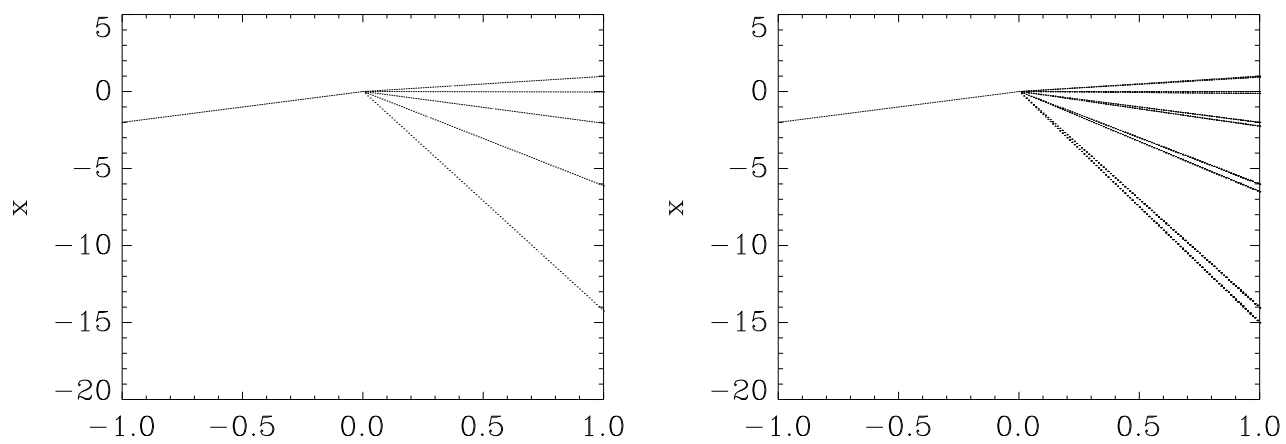

a)

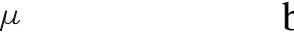

b)
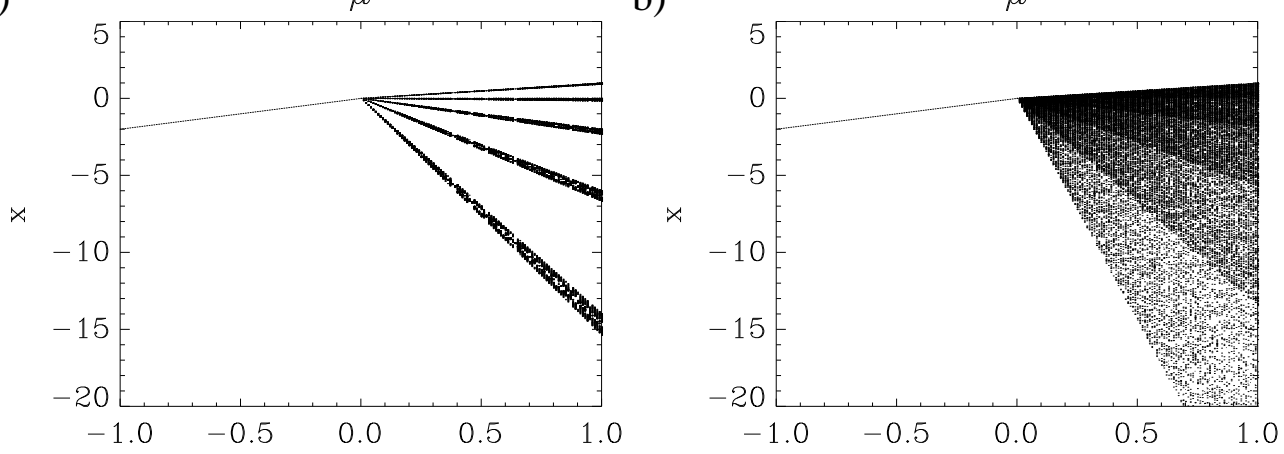

c)

d)

Figure 3.5: Series of border collision bifurcations for the map (3.5) in the parameter area $0<a_{L}<1$, $a_{R}<-1$ (case 3). With $a_{L}=0.5$. Different attractors of the skewed tent-maps appear for $\mu>0$.

a) $a_{R}=15.5$, i.e $\left(a_{L}, a_{R}\right) \in P_{5}$ a period- 1 to period-5 bifurcation occurs for $\mu=0$.

b) $a_{R}=16.05$, i.e $\left(a_{L}, a_{R}\right) \in C 5_{1} 0$ a period-1 to a 10-piece-chaotic attractor bifurcation occurs for $\mu=0$..

c) $a_{R}=16.4$, i.e $\left(a_{L}, a_{R}\right) \in C 5_{5}$ a period-1 to 5-piece-chaotic attractor bifurcation occurs for $\mu=0$.

d) $a_{R}=16.4$, i.e $\left(a_{L}, a_{R}\right) \in C 5_{1}$ a period-1 to one-piece-chaotic attractor bifurcation occurs for $\mu=0$.

merging occurs, is given by:

$$
a_{L}^{2(n-1)} a_{R}^{3}-a_{R}+a_{L}=0 .
$$

Even this n-piece chaotic attractor undergoes a further bifurcation, where the $n$ bands merge and a 1-piece chaotic attractor is born. The bifurcation line was also obtained in [74], given by:

$$
a_{L}^{(n-1)} a_{R}^{2}-a_{R}+a_{L}=0 .
$$

A special bifurcation scenario is connected with the period-2 case. Here attracting chaotic interval cycles of period $2^{m}$ for all $m \in \mathbb{N}$ appear if the parameters are below the region of stable period- 2 orbits. For the parameters $\left(a_{L}, a_{R}\right)$ crossing $(1,-1)$ an interval cycle of period $2^{\infty}$ occurs for $\mu>0$. From this point onwards a inverse period further internal structure inside the chaotic bands in contrast to smooth maps. 
doubling cascade of interval cycles down to a one piece periodic attractor characterizes the dynamic of skewed tent maps.

For this period doubling cascade of interval cycles in [74] two universal scaling constants are derived. $\delta$ can be defined according to (3.1) using $a_{n}=\left(a_{L, n}, a_{R, n}\right)$ at the bifurcation points at any straight line $a_{R}=k\left(a_{R}-1\right)+1$ which passes through $\left(a_{L}, a_{R}\right)=(1,-1)$. It was proven that the limit exists and $\delta=2$. For the scaling in the state space the distance of the merging points $x_{n}$ at the bifurcation point were considered and $\alpha$ was defined, similar to (3.2) by $\alpha=\lim _{n \rightarrow \infty}\left(1-x_{n}\right)\left(1-\left(x_{n+1}\right)\right)$. It appears that the state space scaling not obeys a power law since $\alpha_{n} \sim \alpha_{0} \cdot 2^{n}$ with $\alpha_{0} \simeq \sqrt{2}$.

Thus in a piecewise linear map with one critical point of skewed tent map type the following order of attractor bifurcations occurs:

$$
\begin{aligned}
& (\mathrm{P} 1) \succ P 2 \succ\left(C 2_{2^{m}} \succ C 2_{2^{m-1}} \succ \ldots \succ C 2_{2} \succ C_{I}\right) \\
& \succ P 3 \succ\left(C 3_{6} \succ C 3_{3} \succ C_{I}\right. \\
& \succ P 4 \succ\left(C 4_{8} \succ C 4_{4} \succ C_{I}\right) \ldots \\
& \succ P n \succ\left(C n_{2 n} \succ C n_{n} \succ C_{I}\right) \ldots
\end{aligned}
$$

where the capital letter symbolizes the nature of the attractor (periodic or chaotic), the first superscript denotes the period of the parameter space region it belongs to and the subscript for chaotic attractors denotes the number of chaotic intervals $\left(C_{I}\right.$ means a one piece chaotic attractor). All these attractors emerge, depending on the $a_{L}, a_{R}$ parameters in the border collision bifurcation point in the regions of case 3 and case 1b. See Fig.3.2.2 for examples.

\section{Case 4}

For

$$
-1<a_{L}<0 \text { and } a_{R}<0
$$

every point in $\mathrm{L}$ is mapped to $\mathrm{R}$ and vice versa. Thus all admissible orbits are of $\{10\}^{n}$ $(n \geq 1)$ type. Therefore the dynamics is governed by the second iterate. This second iterate has a stable fixed point at $x^{*}=a_{L}(\mu+1) /\left(1-a_{L} a_{R}\right)$ for $\mu>0$ if $a_{L} a_{R}<1$, and an unstable saddle for $\mu>0$ and $a_{l} a_{R}>1$.

\section{Case 4 a}

Thus we obtain for

$$
-1<a_{L}<0 a_{R}<-1 \text { and } a_{L} a_{R}<1
$$

a period doubling period-1 to period- 2 border collision bifurcation. Note that the linear divergence of the period doubled orbit (Fig.3.6) makes here the visible difference to a period doubling bifurcation which is obtained in smooth maps. 


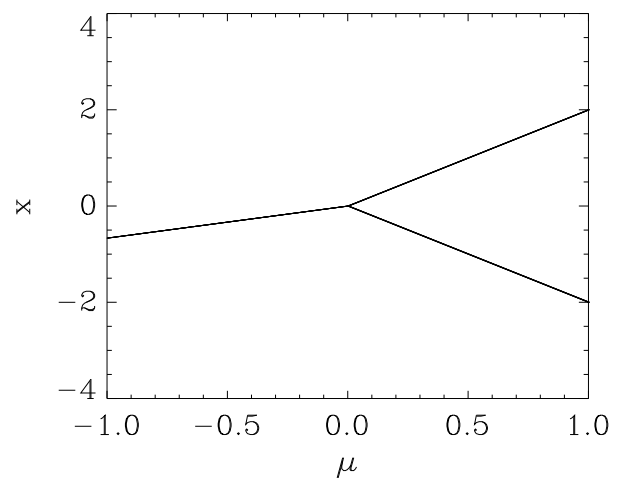

Figure 3.6: Period doubling border collision bifurcation (case 5a) for the map (3.5) with $a_{L}=$ $-0.5, a_{R}=-1.5$. Note that the two branches of the period 2 orbit diverge linear from the bifurcation point and hold a fixed direction thenceforwards. For the usual period doubling bifurcation the diverging branches start perpendicular and the direction of the branches changes continuously behind the bifurcation point.

\section{Case 4 b}

If

$$
-1<a_{L}<0, a_{R}<-1 \text { and } a_{L} a_{R}>1
$$

a bifurcation from period-1 to no attractor appears.

\section{Case 6}

Finally we note that for

$$
a_{L}<-1 \text { and } a_{R}<-1
$$

no attractor for both $\mu<0$ and $\mu>0$ exists.

\subsubsection{Border Collision Bifurcations in Continuous Two Dimensional Maps}

Even in continuous piecewise smooth maps in two dimensions border collision bifurcations take place. Here the border between two segments is a one dimensional manifold. Since this bifurcations were investigated in great detail in recent publications $[7,9,84]$ here only a brief reference should be given, concerning the differences to the one dimensional case and some new phenomena due to the increased dimension of the maps.

A suitable normal form of piecewise linear maps in two dimensions according to [7] 
is given by:

$$
f(x)=\left\{\begin{array}{l}
f_{L}(x)=\left(\begin{array}{ll}
a_{L} & 1 \\
h_{L} & 0
\end{array}\right)\left(\begin{array}{l}
x \\
y
\end{array}\right)+\mu\left(\begin{array}{l}
1 \\
0
\end{array}\right) \text { for } x \leq 0 \\
f_{R}(x)=\left(\begin{array}{ll}
a_{R} & 1 \\
h_{R} & 0
\end{array}\right)\left(\begin{array}{l}
x \\
y
\end{array}\right)+\mu\left(\begin{array}{l}
1 \\
0
\end{array}\right) \text { for } x>0
\end{array}\right.
$$

The fixed points of the left and right branches, which may be virtually ( Sec. 3.2.2) are given by:

$$
\begin{aligned}
L^{*} & =\left(\frac{\mu}{1-a_{L}+h_{L}}, \frac{-h_{L} \mu}{1-a_{L}+h_{L}},\right) \\
R^{*} & =\left(\frac{\mu}{1-a_{R}+h_{R}}, \frac{-h_{R} \mu}{1-a_{R}+h_{R}},\right)
\end{aligned}
$$

Their stability is governed by the eigenvalues $\lambda_{1,2}=\frac{1}{2}\left(h \pm \sqrt{a^{2}-4 h}\right)$. Thus, in two dimensional maps even complex eigenvalues are possible, indicating a spiral fixed point, where the rotation is clockwise with $a>0$ and counterclockwise otherwise.

Since the symmetry with respect to $a_{L}, a_{R}, \mu$ exists for the two dimensional case too, the following considerations are restricted to parameters $a_{L} \leq a_{R}$.

At first the bifurcations for $0<\left|h_{L, R}\right|<1$ are considered. As long as all eigenvalues of the fixed points are real numbers, in principal the same bifurcations as in the 1D case are obtained. Especially the border collision pair bifurcations appear in a similar way, only the conditions for $a_{L, R}$ have to be slightly changed (by replacing 1 by $\left.1+h_{L, R}\right)$ and one has also to consider the values of $h_{L, R}$ to obtain the stability threshold for chaotic attractors (i.e. the line that separates $1 b$ and $1 c$ in Fig.3.2 see [7]). Even here the chaotic attractors are robust.

As long as either $2 \sqrt{h_{L}}<a_{L}<1+h_{L}$ or $2 \sqrt{h_{R}}<a_{R}<1+h_{R}$, or both $a_{L, R}$ are of equal sign and $\left|a_{L, R}\right|<2 \sqrt{h_{L, R}}$ or $0>2 \sqrt{h_{L, R}}>a_{L, R}>1+h_{L, R}$ the border crossing of fixed points leads to unique period 1 orbits for all $\mu \neq 0$ (similar to case 2 in Sec.3.2.2) despite of the fact that the fixed points may be attracting in a spiral way. A rather strange situation, that is not possible in one dimensional maps, occurs if the two fixed points $L^{*}, R^{*}$ are spiral attractors of opposite rotation or a spiral attractor for one branch is combined with a flip saddle for the other branch (despite of the "virtuality" of one of the fixed points). In this case a large number of coexisting attractors may occur, which leads to multiple attractor bifurcations ${ }^{8}$ [43] if $\mu$ crosses zero.

We discuss the possible cases in more detail:

Case m1: multiple attractors both for $\mu<0$ and $\mu>0$ :

\footnotetext{
${ }^{8}$ It was argued in [43] that such multiple attractor bifurcations are a source of an extreme sensitivity to noise, since for a variation of the bifurcation parameter through its critical value in the presence of noise the new orbit of the system may be unpredictable by any means.
} 
If $0<a_{L}<2 \sqrt{h_{L}},-2 \sqrt{h_{R}}<a_{R}<0$ or $0<a_{R}<2 \sqrt{h_{R}},-2 \sqrt{h_{L}}<a_{L}<0$ two spiral attractors with opposite sense of rotation occur. For $-2 \sqrt{h_{L}}<a_{L}<2 \sqrt{h_{L}}$, $-2 \sqrt{h_{R}}>a_{R}>-\left(1+h_{R}\right)$ a spiral attractor and a flip attractor occur. In this case there are multiple attractors both for $\mu<0$ and $\mu>0$ existing, one of them is the fixed point $L^{*}$ or $R^{*}$.

Case m2: multiple attractors for $\mu<0$ :

If $a_{R}<-\left(1+h_{R}\right)$ and $-2 \sqrt{h_{L}}<a_{L}<2 \sqrt{h_{L}}$ then there can be multiple attractors for $\mu<0$. Depending on $a_{L}$ the border collision bifurcation at $\mu=0$ is of type

multiple attractors to no attractor

$\left(-2 \sqrt{h_{L}}<a_{L}<0\right.$ and $\left.1-a_{L} a_{R}+h_{L}+h_{R}+h_{l} h_{R}<0\right)$,

multiple attractors to period-2

$\left(-2 \sqrt{h_{L}}<a_{L}<0\right.$ and $\left.1-a_{L} a_{R}+h_{L}+h_{R}+h_{l} h_{R}>0\right)$, or

multiple attractors to chaotic or periodic attractors

$\left(0<a_{L}<2 \sqrt{h_{L}}\right)$.

In the latter case the occuring attractors for $\mu>0$ are the two dimensional counterparts of the skewed tent-map families attractors discussed as case 3 in 3.2.2.

If both $-1<h_{L, R}<0$ there are no imaginary eigenvalues for the fixed points. Therefore the above discussed multiple attractor phenomenon does not occur and only the border collision types which are known from one dimensional maps are possible. For further details see [7,9].

A special situation is given if either $h_{L}=0$ or $h_{R}=0$. Then the map is two dimensional at one side, but one dimensional at the other side of the border. This situation is studied in some extend in [84]. The authors end up with a collection of catalogues for twenty distinguishable regions in parameter space with different border collision bifurcations for $h_{L}>1, h_{R}=0$ and another twenty regions for $0<h_{L}<1, h_{R}=0$ as well as twelve regions for $-1<h_{L}<0, h_{R}=0$. In the cases with $|h|>1$ inside the regions with basically the same behavior, i.e. the same type of fixed points for $\mu<0$ and $\mu>0$ occurs, depending on the parameters chaotic and periodic attractors. Such chaotic attractors can, due to the special nature of piecewise smooth maps be organized around repellers and need not to be associated with saddle type fixed points [84].

This brief review may already show, that the class of border collision bifurcations is even for the case of only two segments in a continuous map extremely rich and there may be new phenomena in dimensions larger than two. To our knowledge no systematic investigation of such higher dimensional piecewise linear maps was done up to now. 


\subsection{Bifurcations of Piecewise Linear Maps with a Discontinuity}

In the following we give an introduction to phenomena associated with two segment piecewise linear maps containing a discontinuity or, in other words, a jump. Since even a discontinuity point between two segments can be seen as a border the bifurcation of such discontinuous maps are also summarized under the term border collision bifurcations. As a suitable normal form we use the following map, which was proposed recently in [109].

$$
x_{n+1}=\left\{\begin{array}{lll}
a_{L} x_{n}+\mu & \text { for } \quad x_{n} \leq 0 \\
a_{R} x_{n}-(l-\mu) & \text { for } \quad x_{n}>0
\end{array}\right.
$$

This map consist of two linear segments, denoted by $\mathrm{L}$ and $\mathrm{R}$ with slopes $a_{L}$ and $a_{R}$, respectively and a discontinuity at $x=0$. The height of the discontinuous jump at $x=0$ is $l$. We consider the parameter $\mu$ as bifurcation parameter. Hence we normalize $l$ by $l=1$ for $\left|a_{L}\right|<1,\left|a_{R}\right|<1$. For all $l>0$ in this cases a linear scaling for attractors and periodic orbits for $\mu \neq 0$ applies. For slopes $\left|a_{L, R}\right|>1$ a normalization of the discontinuous jump to one is not sufficient. However, even there we assume $l=1$ since a choice has to be made.

As for the continuous case discussed in the foregoing Sec. 3.2 a symmetry in the parameter space with respect to $a_{L}, a_{R}, \mu$ exists. If a certain orbit occurs for $a_{R}<$ $a_{L}, \mu$ the same orbit is an orbit of $a_{L}^{\prime}=a_{R}, a_{R}^{\prime}=a_{L}$ and $\mu^{\prime}=(1-\mu)$. Therefore we shall consider in the following the attractors and bifurcations of map (3.16) in the parameter range $a_{L}>a_{R}$.

\subsubsection{General Properties of Periodic Orbits}

If $\left|a_{L}\right|<1$ and $\left|a_{R}\right|<1$ the jump impedes the existence of fixed points in the map (3.16) if $0<\mu<1$. Thus we conclude that a periodic orbit has to be of period 2 or larger. Every periodic orbit that exists is also stable since $|d f / d x|<1$ for all $x$. We therefore have to find the admissible periodic orbits with respect to $\left(a_{L}, a_{r}, \mu\right)$ to characterize the dynamics of the piecewise linear map with one discontinuity and two segments. As mentioned in [109] the disposition of periodic orbits with respect to $p$ obeys an asymmetric devil's staircase, that may be incomplete in some cases.

The common feature of maps (3.16) with $0<a_{L}<1$ is a channel formed by the left segment of the map and the diagonal $x_{n+1}=x_{n}$ (see Fig.3.12). Once injected from the right into the channel, a trajectory spends a number of iterations inside until it can leave the channel through the draining interval $D_{L}:\left(x_{n} \in\left(f_{L}^{-1}(0), 0\right)\right)$. The injection takes place inside an injection interval $I_{L}$. If $1>a_{R}>0$ even the right segment forms a similar channel.

The value of $\mu$ determines the width of the channel. A change in the channel width by 


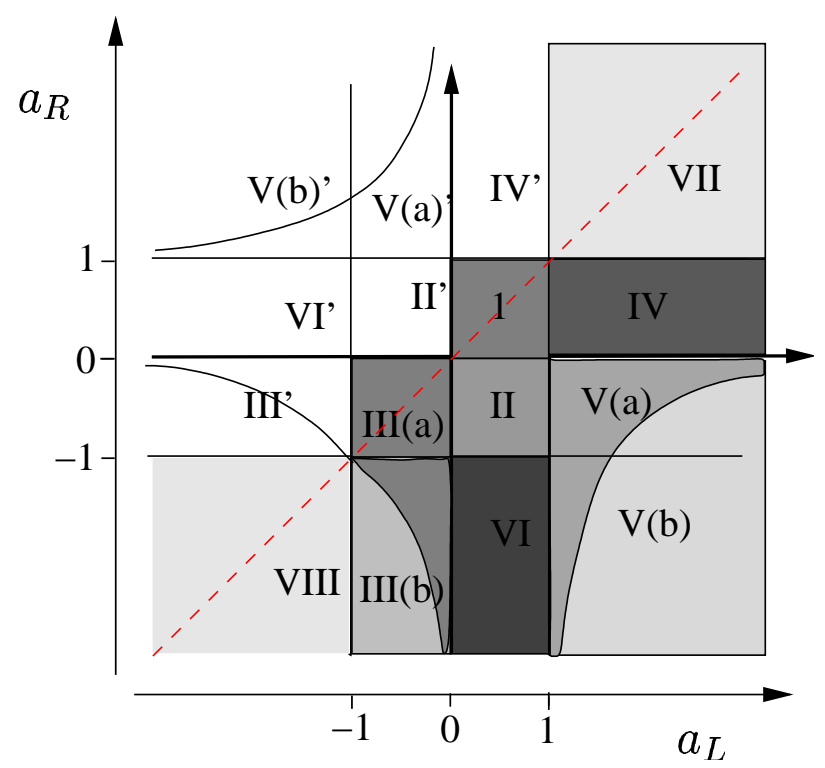

Figure 3.7: Overview of the $a_{L}, a_{R}$ parameter space of piecewise linear maps with two segments and a discontinuity Eq.(3.16), showing regions with the same qualitative bifurcation phenomena. The labeling of the regions are the cases that appear in the text. The primed numbers show regions with the same bifurcation phenomena for an inverse border crossing due to the symmetry indicated by the dashed line.

changing $\mu$ can result in bifurcations if the number of iterations inside the channel is altered.

Now, in principle the conditions for the existence of a periodic orbit with a given symbolic encoding $\left\{J_{i}\right\}$ can be investigated by direct analytic calculus since an orbit has to fulfill the equation

$$
f_{R} \circ \ldots \circ f_{L}\left(x_{1}\right)=x_{1}
$$

with the left hand side ordered according to the symbolic encoding of the orbit and $x_{1}$ is the leftmost point of the orbit. Because this equation is linear with respect to $x_{1}$ its solution is unique. A number of restrictions concerning the points $\left\{x_{i}\right\}$ of the orbit are necessary. Assumed, we have a block of $m$ consecutive $f_{R}$ 's followed by $n$ consecutive $f_{L}$ 's, i.e. an orbit of $\left(0^{n} 1^{m}\right)$ type. Then the set of inequalities

$$
f_{L}^{n-1}\left(x_{1}\right)<0<f_{L}^{n}\left(x_{1}\right), f_{R}^{m-1} f_{L}^{n}\left(x_{1}\right)<0<f_{R}^{m} f_{L}^{n}\left(x_{1}\right)
$$

must be fullfilled. A similar set can be used for every, even more complicated periodic orbit. This set of inequalities gives a sufficient condition for the existence of a periodic orbit of the desired form, which can be written as:

$$
\mu_{\text {min }}\left(\left\{J_{i}\right\}, a_{L}, a_{R}\right)<\mu<\mu_{\max }\left(\left\{J_{i}\right\}, a_{L}, a_{R}\right) .
$$




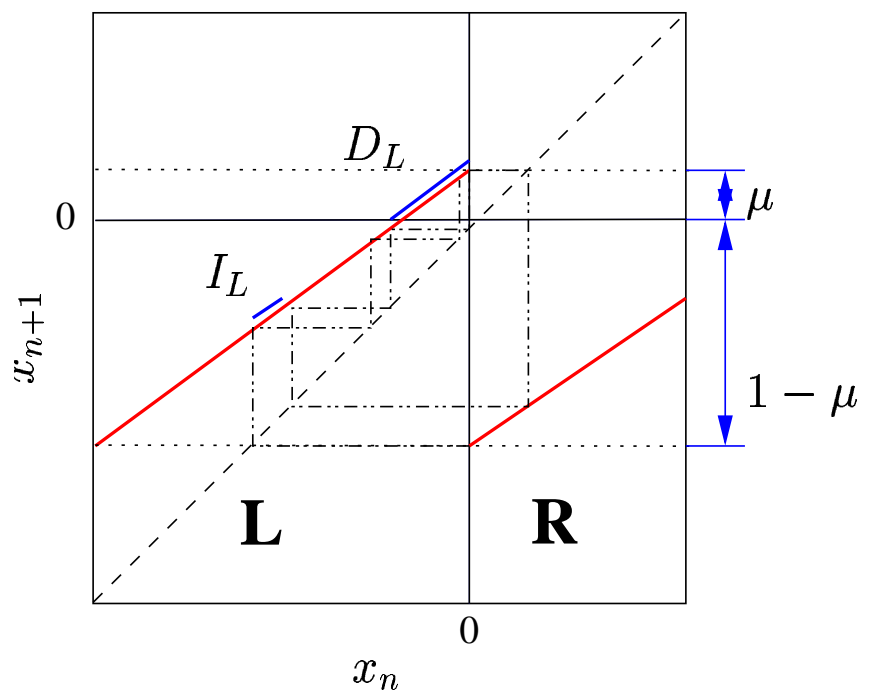

Figure 3.8: Example of a piecewise linear map Eq.(3.16) with a discontinuity at $x=0$. Here the situation $0<a_{L}<1$ and $0<a_{R}<1$ is depicted, which corresponds to case 1 in Fig. 3.7. The injection interval $I_{L}$ and the draining interval $D_{L}$ of the channel formed by the left segment of the map are indicated as well as the bifurcation parameter $\mu$.

However, the stability of this orbit has to be considered separately. For an unique period-2 orbit $\{10\}$ this method leads to

$$
\frac{a_{L}}{1+a_{L}}<\mu<\frac{1}{1+a_{R}} .
$$

This condition for a period-2 holds for all $a_{L}, a_{R}$. For $\left|a_{L}\right|,\left|a_{R}\right|<1$ the stability of the period- 2 orbit is ensured and the interval given by (3.18) includes always $\mu=1 / 2$. Now we shall consider the appearance of periodic orbits with respect to $\left(a_{L}, a_{R}\right)$ in more detail.

\subsubsection{Period Adding Scenarios}

\section{Case I}

In the parameter region

$$
1>a_{L}>a_{R}>0
$$

the most rich variety of periodic orbits can be obtained. At first we consider periodic orbits with reinjection number $N=1$ i.e period $n>2$ orbits of the type $\left\{1^{n-1} 0\right\}$ and $\left\{10^{n-1}\right\}$. The condition for a $\left\{1^{n-1} 0\right\}$ period- $(n+1)$ orbit (winding number $r=$ $n-1 / n$ ) was derived in [109] by:

$$
\frac{1-a_{R}^{n-2}+a_{L} a_{R}^{n-2}\left(1-a_{R}\right)}{1-a_{R}^{n-1}+a_{L} a_{R}^{n-2}\left(1-a_{R}\right)}<\mu<\frac{1-a_{R}^{n-1}}{1-a_{R}^{n}} .
$$




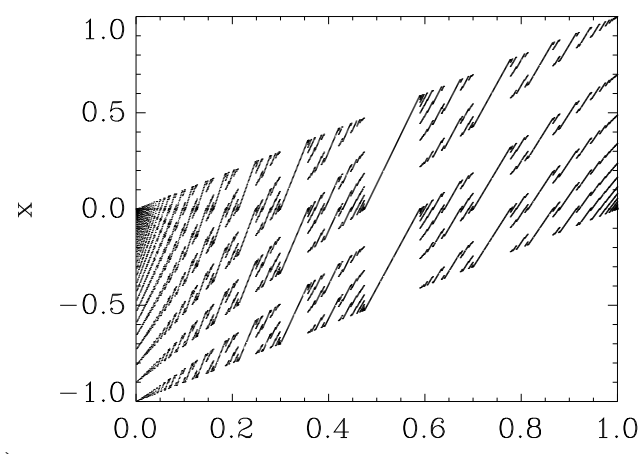

a)

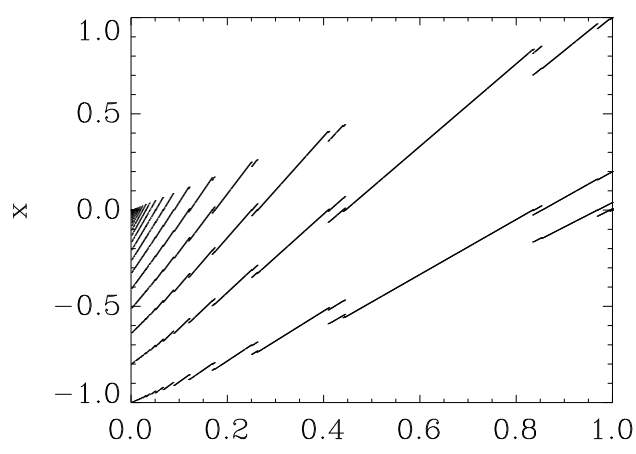

c)

Figure 3.9: Example of period adding scenarios of map Eq.(3.16) in the case 1. a) shows the bifurcation diagram for $0<\mu<1$ and $a_{L}=0.9, a_{R}=0.7$ and $\mathrm{b}$ ) the corresponding devil's staircase of winding numbers defined according to Eq.(3.4). c), and d) depicts the period adding scenario and the winding numbers, respectively, for $a_{L}=0.8, a_{R}=0.2$. Note that for $\mu=0.5$ in all cases a period-2 (10) orbit exists.

In a similar way one obtains for a $\left\{10^{n}\right\}$ period- $(n+1)$ orbit (winding number $r=$ $1 / n+1)$ :

$$
\frac{a_{L}^{n-1}\left(1-a_{L}\right)}{1-a_{L}^{n}}<\mu<\frac{a_{L}^{n-2}\left(1-a_{L}\right)}{1-a_{L}^{n-1}+a_{R} a_{L}^{n-2}\left(1-a_{L}\right)} .
$$

From (3.19) and (3.20) the following order of intervals for any fixed $\left(a_{L}, a_{R}\right)$ is found:

$$
\begin{gathered}
\mu_{\min }\left(10^{n+1}\right)<\mu_{\max }\left(10^{n+1}\right)<\mu_{\min }\left(10^{n}\right)<\mu_{\max }\left(10^{n}\right)<\ldots<\mu_{\max }\left(10^{2}\right)<\mu_{\min }(10)< \\
\mu_{\max }(10)<\mu_{\min }\left(1^{2} 0\right)<\ldots<\mu_{\min }\left(1^{n} 0\right)<\mu_{\max }\left(1^{n} 0\right)<\mu_{\min }\left(1^{n+1} 0\right)<\mu_{\max }\left(1^{n+1} 0\right)
\end{gathered}
$$

Thus, for $\mu_{\max }\left(10^{n+1}\right)<\mu<\mu_{\min }\left(10^{n}\right)$ there is no $n$ such that (3.20) can be fullfilled, therefore orbits of a more complicated structure must exist in these "gaps" between the $\left\{10^{n}\right\}$ orbits.

Inbetween these gaps the draining interval contains subsets of both $f_{L}^{n}\left(I_{L}\right)$ and $f_{L}^{n+1}\left(I_{L}\right)$. The periodic orbits arising in $\mu_{\max }\left(10^{n}\right)<\mu<\mu_{\min }\left(10^{n+1}\right)$ hence are build only from subsequences of $\left(10^{n}\right)$ and $\left(10^{n+1}\right)$ type. By a deeper study it is possible to ob- 

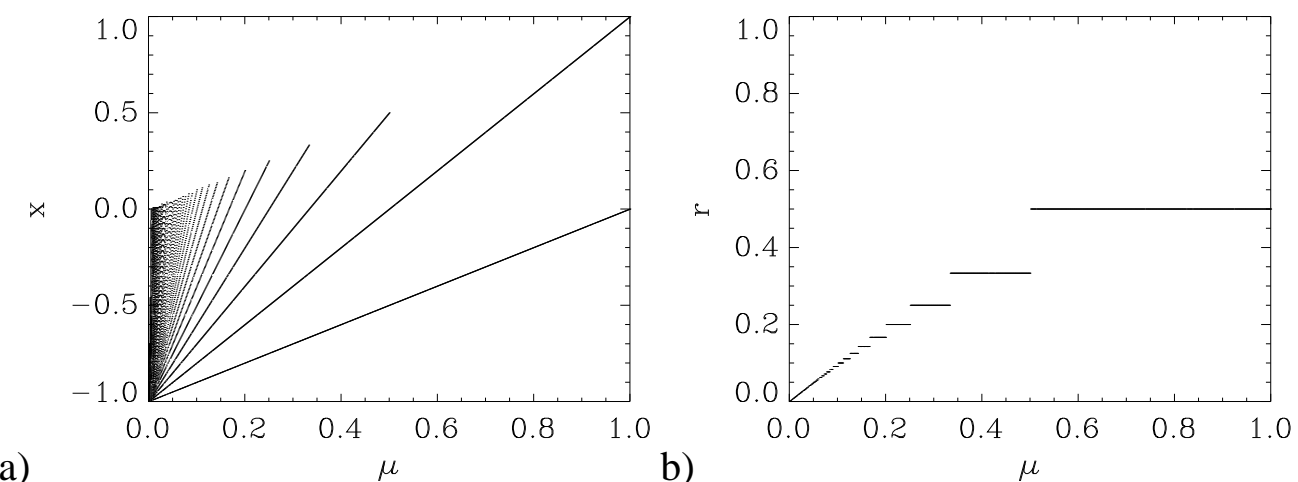

Figure 3.10: Bifurcation diagram of the period increment scenario in the non generic case $a_{L}=$ $1.0, a_{R}=0.0$.

tain a Farey tree like ordering of the existing periodic orbits. This was done for a special case in [99]. However, the structure of this symbolic period adding tree crucially depends on $a_{L}, a_{R}$. Without going into deeper analysis we briefly remark, that not for every reinjection number a orbit exists and basically the symbolic period adding tree is finite. However, higher and higher periodic orbits are obtained in smaller and smaller intervals and and therefore the periodicity varies non- monotonic (Fig.3.9).

\subsubsection{Period Increment Scenarios}

\section{Case II}

In the case where the right segment has a slope of zero or negative slope, i.e.

$$
-1<a_{L} \leq 0 \text { and } 0<a_{R}<1
$$

the situation is lucid compared to the previous considerations.

To begin with, we shall consider the special case $a_{R}=0, a_{L}>0$. Here the injection interval shrinks to one point given by $x=\mu-1$. Obviously all existing orbits, even for $a_{L}>1$ are superstable since the contraction of the right segment is infinite, and all are of the symbolic form $\left(10^{n}\right)$. Using (3.20) it can be determined that $\mu_{\max }\left(10^{n}\right)=\mu_{\min }\left(10^{n+1}\right)$ and thus a period-n orbit occurs ${ }^{9}$ if:

$$
\frac{a_{L}^{n-1}\left(1-a_{L}\right)}{1-a_{L}^{n}}<\mu_{n}<\frac{a_{L}^{n}\left(1-a_{L}\right)}{1-a_{L}^{n}+1} .
$$

Here, the period of the system will be increased at every bifurcation point by one and its order is represented by an arithmetical series. Due to this fact and to make a difference to the period adding scenario discussed above it was suggested in [6] to call this situation a period increment scenario. For $0<a_{L} \leq 1$ this period increment is

\footnotetext{
${ }^{9}$ For $a_{R}=0, a_{L}=1$ a simple geometrical consideration yields: $\frac{1}{n-1}<\mu_{n} \leq \frac{1}{n}$
} 


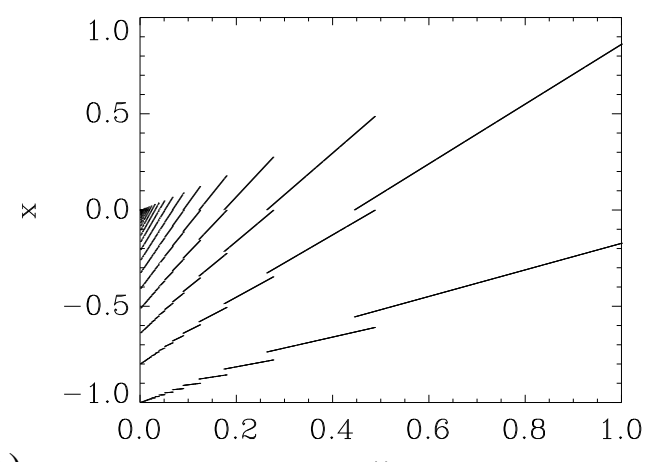

a)

Figure 3.11: Bifurcation diagram (a) and corresponding winding numbers (b) for a period increment scenario (case II). The parameters $a_{L}=-0.8, a_{R}=0.2$ are used.

complete, i.e. all $n \in \mathbb{N}$ appear for $\mu \rightarrow 0$. Thus we can compute scaling constants for this scenario using (3.1) and (3.2). For $\delta$ we derive:

$$
\begin{aligned}
& \delta=\lim _{n \rightarrow \infty} \frac{\mu_{m i n, n-1}-\mu_{m i n, n}}{\mu_{m i n, n}-\mu_{m i n, n+1}}=\lim _{n \rightarrow \infty} \frac{a_{L}-a_{L}^{n}}{1-a_{L}^{n}}=a_{L} \\
& \alpha=\lim _{n \rightarrow \infty} \frac{\left.\left(a_{L}(\mu-1)+2 \mu-1\right)\right|_{\mu_{m i n, n}}}{\left.\left(a_{L}(\mu-1)+2 \mu-1\right)\right|_{\mu_{m i n, n+1}}}=\lim _{n \rightarrow \infty} \frac{a_{L}^{-1}-a_{L}^{n}}{1-a_{L}^{n}}=1
\end{aligned}
$$

For $0>a_{R}>-1$ (and $0<a_{L}<1$ ) even no consecutive 1's in a symbolic orbit are possible, because every point in $\mathrm{L}$ is mapped to $\mathrm{R}$ within the next iteration. By application of the above mentioned method the following condition for the existence of an exclusive $\left\{10^{n}\right\}$ orbit is obtained [109]:

$$
\frac{a_{L}^{n}\left(1-a_{L}\right)}{1-a_{L}^{n+1}+a_{L}^{n} a_{R}\left(1-a_{L}\right)}<\mu<\frac{a_{L}^{n-1}\left(1-a_{L}\right)}{1-a_{L}^{n}}
$$

The borders for the unique $10^{n}$ orbit are, if we note $\mu_{\min }^{*}(n)<\mu^{*}<\mu_{\max }^{*}(n)$, $\mu_{\min }^{*}(n)=\mu_{\max }(n+1)$ and $\mu_{\max }^{*}(n)=\mu_{\min }(n-1)$. This relation is caused by the negative slope of the right segment, which interchanges the upper and lower boundary of the draining interval $D_{L}$ while it is mapped by $f_{r}$ to the injection interval $I_{L}$. Moreover, the existence of a stable $\left\{10^{n}\right\}$ orbit is determined again by (3.20). Thus inside the interval $\mu_{\max }^{*}(n+1)<\mu<\mu_{\min }^{*}(n)$ both stable $\left\{10^{n+1}\right\}$ and $\left\{10^{n}\right\}$ periodic orbits are coexisting. Orbits with injection numbers $N>1$ are not obtained in this case. As a consequence one obtains an incomplete devil's staircase containing only winding numbers that result from orbits $\left\{10^{n}\right\}$ where intervals of unique period $n$ orbits are connected by intervals where the two neighboring periodic orbits are interleaving (see Fig.3.11). 


\section{Case III}

If both the slope of the right and the left segment are negative and contracting

$$
\left(-1<a_{L}<0 \text { and }-1<a_{R}<0\right),
$$

the only possible stable orbit is the period- $2\{10\}$ orbit.

\section{Case III a}

This stable period-2 orbit even remains stable for all $\mu>0$ for $a_{R}<-1,0>a_{L}>-1$ as long as $a_{R}>a_{L}^{-1}$.

\section{Case III b}

If, on the other hand $a_{R}<a_{L}^{-1}$ (with $0>a_{L}>-1$ ) no stable attractor still exist for all $\mu$.

\subsubsection{Discontinuous Maps with Slopes Larger than One}

Now we shall consider the dynamics of the map (3.16) if $\left|a_{L}\right|>1$ or $\left|a_{R}\right|>1$. If the slope of the left segment is larger than one, for every $\mu$ an unstable fixed point at the left branch exists, and if $a_{R}>1$ also an unstable fixed point on the right branch is found. These fixed points are given by:

$$
L^{*}=(\mu) /\left(1-a_{L}\right) \quad \text { and } \quad R^{*}=(\mu-1)\left(1-a_{R}\right)
$$

For the normalized $l=1$ in Eq.(3.16). Every trajectory, that contains a point located left of $L^{*}$ diverges towards $-\infty$ and every trajectory with a point right of $R^{*}$ diverges towards $\infty$ (given that $a_{L}, a_{R}>1$ ).

\section{Case IV}

For

$$
a_{L}>1 \text { and } 0<a_{R}<1
$$

the equations (3.19) and (3.20) for the existence of stable periodic orbits remain true as long as the associated periodic orbit exists. For $a_{L}>1$ the region of existence of any periodic orbit shrinks with increasing $a_{L}$ up to the moment where $\mu_{\min }(n)=\mu_{\max }(n)$. This point is reached with $a_{L}^{n}=a_{R}^{-1}$ for $\left\{10^{n}\right\}$ orbits and for $a_{L}^{-1}=a_{R}^{n}$ for $\left\{1^{n} 0\right\}$ orbits, i.e. just in the point, where the periodic orbit also looses its stability. Even for more complicated periodic orbits, occuring in the period adding scenario a condition of this form can be derived since the devil's staircase obeys a strictly increasing order of occurrence for the winding numbers with increasing $\mu$. Thus for every $a_{L}, a_{R}$ a critical winding number $r_{c}=c /(c+1)$ with $c=\ln \left(a_{R}\right) /\left|\ln \left(a_{L}\right)\right|$ and a critical $\mu_{c}$ exists. If $\mu>\mu_{c}$ a period adding scenario as in case I occurs, leading to stable periodic orbits. For $\mu<\mu_{c}$ all periodic orbits are unstable. Then chaotic attractors 
that may consist of several intervals occur. The stable chaotic attractor disappears if $f_{r}\left(0^{+}\right)<L^{*}$ i.e.

$$
\mu \leq \frac{a_{L}-1}{a_{L}} .
$$

\section{Case V}

A similar situation arises if

$$
a_{L}>1 \text { and } a_{R}<0 .
$$

In this case a period increment scenario with regions of coexisting $\left\{10^{n}\right\}$ and $\left\{10^{n+1}\right\}$ orbits arises as in case II. The regions of coexisting orbits expand for increasing $a_{L}$. If a periodic orbit looses its stability $a_{L}^{n}=a_{R}-1$ chaotic orbits appear. But in regions where a second periodic attractor coexists, that has, due to an other periodicity not lost its stability the chaotic attractor eventually is not obtained. If all coexisting period orbits in a given parameter region are unstable chaotic attractors are formed. Even here interval attractors may occur. This chaotic orbits clearly loose their stability if $f_{R}(\mu)<L^{*}$, i.e

$$
\mu \geq \frac{a_{L}-1}{1+\left(a_{L}-1\right)\left(a_{R}+1\right)}
$$

For

$$
a_{R}<2\left(1-a_{L}\right) /\left(a_{L}-1\right)
$$

the condition (3.27) requires values of $\mu>1$ to obtain a stable chaotic orbit. In consequence no stable chaotic attractor is possible for a map (3.16) with jump size of one. For jumps larger than one this border becomes more and more restrictive, whereas this condition resembles (3.8) for a vanishing jump at $x=0$. The condition for the disappearance of periodic orbits is given by the inequality $x_{1} \leq L^{*}$ where $x_{1}$ is the leftmost point of the periodic orbit.

Because of the coexistence of period-n and period- $(n+1)$ orbits in the associated parameters regions two coexisting basins occur, where one is the basin of a stable periodic orbit of period $\mathrm{n}$ and the other is the basin of a diverging solution.

\section{Case VI}

Now we shall investigate the case

$$
0<a_{L}<1 \text { and } a_{R}<-1 .
$$

At first the appearance of periodic orbits should be considered. Here again the condition (3.24) with respect to $\mu$ for the existence of an unique $\left\{10^{n}\right\}$ orbit applies. As one easily obtains the parameter interval for such a unique period-n orbit shrinks with decreasing $a_{R}$. On the other hand the intervals where two of this orbits coexist grow with decreasing $a_{R}$ and for smaller values this intervals came in contact and create 

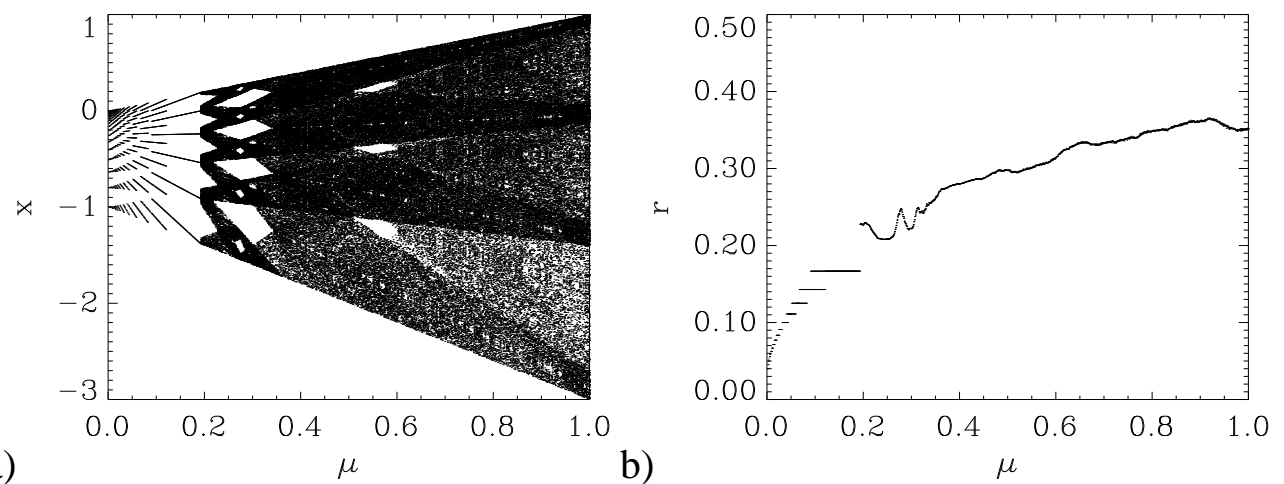

Figure 3.12: Bifurcation diagram and winding numbers for map (3.16) with $a_{L}=0.8, a_{R}=-3.0$, i.e. case VI

intervals where three, or even more periodic orbits can coexist. Inside such intervals orbits of $\left\{10^{n}\right\},\left\{10^{n-1}\right\}, \ldots$ are found.

However, every periodic orbit of $\left\{10^{n}\right\}$ type becomes unstable if $a_{L}^{n} \geq\left|a_{R}^{-1}\right|$.

Let $N$ be the smallest integer with $N>-\ln \left|a_{R}\right| / \ln \left|a_{L}\right|$. Then every periodic orbit $\left\{10^{n}\right\}$ with $n<N$ is unstable, and the critical $\mu_{c}$ is given by the largest $\mu$ for which the stable $10^{N}$ orbit exists, i.e.:

$$
\mu_{c}=\frac{a_{L}^{N-1}\left(1-a_{L}\right)}{1-a_{L}^{N} a_{R} a_{L}^{N-1}\left(1-a_{L}\right)} .
$$

For $\mu>\mu_{c}$ chaotic attractors appear. Those may be consisting of several intervals.

\section{Case VII}

For

$$
a_{L}>1 \text { and } a_{R}>1
$$

we obtain a expanding shift map with generally two different slopes. This map has a stable chaotic attractor if $\mu \leq R^{*}$ and $\mu-l \geq L^{*}$ i.e.:

$$
\frac{a_{L}-1}{a_{L}} \leq \mu \leq \frac{1}{a_{R}}
$$

This requires $a_{L} /\left(a_{L}-1\right) \geq a_{R}$ to obtain a chaotic attractor.

\section{Case VIII}

Finally we remark, that for

$$
-1>a_{L} \text { and }-1>a_{R}
$$

no stable attractor for the map 3.16 exists. 


\section{Attractors of piecewise linear one dimensional maps with one discontinuity and} a positive jump

\begin{tabular}{|cc|}
\hline$a_{L}, \mathrm{a}_{R}$ & attractor \\
\hline$\left|a_{L}\right|<1,\left|a_{R}\right|<1$ & $\begin{array}{c}\text { two coexisting stable fixed points } \\
\text { one in } \mathrm{L}, \text { attracting in } \mathrm{L} \text { and one in } \mathrm{R} \text {, attracting in } \mathrm{R}\end{array}$ \\
\hline$a_{L}>1,\left|a_{R}\right|<1$ & $\begin{array}{c}\text { one stable fixed point in } \mathrm{R} \\
\text { attracting in } \mathrm{R}\end{array}$ \\
& $\begin{array}{c}\text { one stable fixed point in } \mathrm{L} \\
\text { everywhere attracting }\end{array}$ \\
\hline$a_{L} \mid<1, a_{R}<-1$ & no stable attractor \\
$a_{L}>1, a_{R}>1$ & for $\left(1-a_{L}\right)\left(a_{L}\right) \leq \mu \leq-1 / a_{R}$. (Shift map) \\
\hline$a_{L}<-1, a_{R}<-1$ & chaotic attractor \\
\hline
\end{tabular}

Table 3.1: Overview of fixed points and attractors in piecewise linear one dimensional maps with two segments (Eq.3.16). Basically for $l=-1,-1<\mu<0$ no bifurcations with changing $\mu$ take place.

\subsubsection{Positive Jump at the Discontinuity}

For the sake of completeness we have summarized the behavior of the map (3.16) for $-1<\mu<0, l=-1$ in table 3.1. In this case the map jumps at $x=0$ from a value smaller than zero towards a positive one. For the the map in a large fraction of the $\left(a_{L}, a_{R}\right)$ parameter space simply stable fixed points exists, only for both $a_{L}<-1$ and $a_{R}<1$ a Bernoulli type shift map as in case VII is obtained, but now with two negatively sloped segments.

\subsection{Summary}

We have demonstrated, that the bifurcations of piecewise linear maps in one dimension are very distinct to the well known bifurcations of smooth one dimensional maps (as the logistic map, for instance). The bifurcations that are caused by non-smoothness are summarized under the notion border collision bifurcations. Basically the type of bifurcation depends on the slopes of linear segments. For continuous piecewise linear maps with two segments five regions of qualitative distinct bifurcation behavior are found with respect to a symmetry regarding the interchange of slopes (Sec.3.2). If additional a discontinuity in the map is considered, further eight regions of different 
bifurcation behavior are identified (Sec.3.3). The cases, that appear at the borders of the above discussed regions in parameter space are not included here.

Whereas most of the results we have reviewed here appeared in the literature at distinct places in various contexts a comprehensive overview of this class of bifurcations however, is to our knowledge not available yet.

This chapter therefore provides a discussion of such bifurcations that play a major rule in the next chapters of this thesis. The nature of hybrid systems implies nonsmooth flows. Border collision bifurcations are therefore generic in hybrid systems. A research on hybrid systems or other non-smooth dynamical systems may stimulate investigations of border collision bifurcations in dimensions larger than one. Whereas the bifurcations of continuous piecewise linear maps in two dimensions are well understood meanwhile (see Sec.3.2.3), a systematic investigation of discontinuous piecewise linear two dimensional maps was not carried out yet, for instance.

The hybrid systems consisting of tanks and servers, that switch between this tanks are model systems for the appearance of border collision bifurcations. This rich class of bifurcations basically structures the variety of dynamical behaviors in this systems. 


\title{
4 Switched Tank Hybrid Systems
}

\begin{abstract}
In this chapter the dynamics of hybrid systems consisting of servers that switch between tanks according to some logical rules is investigated. In Sec. 4.2 we introduce two examples of such systems, the switched arrival and switched server system including maximum capacities of the tanks.The framework of strange billiards is derived and limiting cases for the dynamics are considered. These limiting cases clarify the close relation between these two models. In Sec. 4.3 the dynamics of systems with three thanks in between the limiting cases will be considered in some detail.

In the second part of this chapter (Sec.4.4) related models are briefly discussed. This includes modified switching rules (Sec.4.4.1), switching thresholds that are applied to only one tank (Sec.4.4.2) and a model that involves two switching servers and has a hybrid Poincaré map (Sec.4.4.3). Finally, in Sec. 4.5 we draw some conclusions.
\end{abstract}

\subsection{Switching Between Tanks}

In the present work we investigate a certain class of hybrid systems consisting of basic units, called tanks in the following, that are driven by discrete events. Although the two tank system (see Sec.2.1.1) is frequently used as standard example in hybrid systems literature, not much is known up to now about the dynamics of such systems, especially if the systems contain more than two tanks and additional switching thresholds. Hence we try to fill this gap and end up with a number of dynamic phenomena (as, for instance, a variety of border collision bifurcations) in these remarkably simple systems.

In the following the tanks $i=1, \ldots, n$ have maximum capacities $b_{i}, i=1,2, \ldots, n$ and can be continuously filled with fluid at rates $\lambda_{i}$ and emptied with rates $\mu_{i}$. The fluid content $x_{i}$ of each tank is controlled by switching the inflow or outflow according to switching rules if a tank produces a discrete point event, which means for instance it is full $\left(x_{i}=b_{i}\right)$ or empty. The complete state in state space for connected systems of the described type contains both, continuous variables $x_{i}$ and a discrete (symbolic) variable $q$ labeling the discrete state of the system (i.e., the on or off state of inflows or outflows). At the discrete event times $t_{m}$ the complete state of the system changes $\left[x\left(t_{m}\right), q\left(t_{m}\right)\right] \mapsto\left[x\left(t_{m}\right), q\left(t_{m}^{+}\right)\right]$(with $x=\left(x_{1}, x_{2}, \ldots, x_{n}\right)$ ). 
Due to their behavior that provides a uniform motion inside simplexes if projected in the continuous part of the state space the eidetic term strange billiard was created [92] to label these systems. As we will see the systems have not much in common with ordinary billiard systems. However, such systems can be studied by means of Poincaré mappings. Chaos can be induced or avoided through different shapes of the boundary.

Almost all previous work [25, 92, 98, 59, 63, 62, 87] emphasized the occurrence of switched tank systems in manufacturing systems (see Chap.5 for a discussion). First models of the described type have been analyzed by Chase et al. [25]. They studied two discretely controlled variable systems which occur as limiting cases for the later discussed three tank switched server and three tank switched arrival system, respectively. The first system was shown to be periodic in contrast to the second, which was shown to be chaotic. A three tank switched arrival system with equal rates for all tanks $\left(\lambda_{1,2,3}=1 / 3\right)$ and upper thresholds was studied in [59] by a graphical method, but no deeper analysis of the dynamics was done. In [98] switched server systems under so called corridor policies were considered and the possibility of chaotic behavior for these systems was concluded. The work of Chase et al. was extended by Schürmann and Hoffmann [92] who derived the invariant measure for the $n$-tank switched arrival system with unrestricted tank capacities. Recently the switched arrival system without upper thresholds was studied in $[63,62]$ in the context of discrete material flows and used in [87] to study the interaction of parallel layers of switched arrival systems, coupled through production losses as a model of manufacturing systems.

\subsection{Switched Arrival and Switched Server Systems}

Consider a system consisting of $n$ parallel tanks, and one server as shown in Fig.4.1. At a time, the server can be attached to one tank, only. This server has either to empty all tanks (Fig. 4.1a), in which case they are assumed to fill themselves continuously. Or the tanks, which are all emptied continuously, will be filled by a single switching server (Fig. 4.1b). We call, according to [25], the first situation a switched server system, and the second, where the input to parallel tanks is delivered by a single server, a switched arrival system.

The discrete (symbolic) variable $q$ labels the discrete state of the system (i.e., the position of the switching server).

The switching policies under consideration are the following. For the switched server 
a)

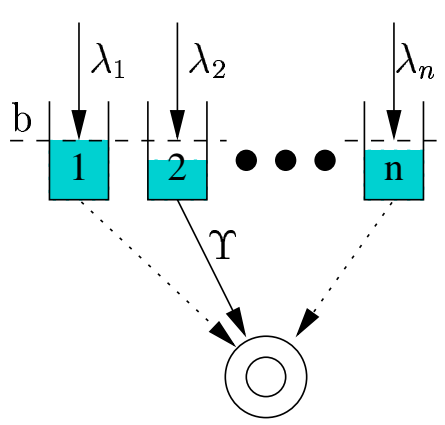

b)

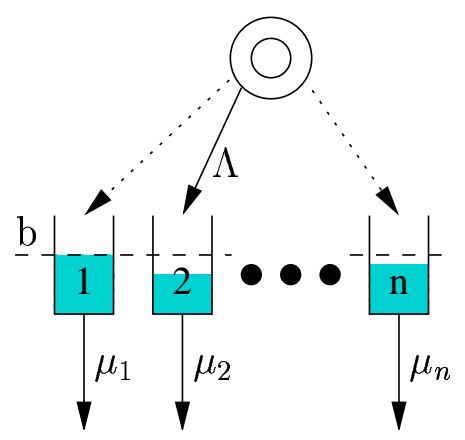

Figure 4.1: Scheme of the $n$-tank switched server system (a) and the $n$-tank switched arrival system.

system:

If a tank is filled to its maximum the server instantaneously starts to serve this tank.

However if the currently served tank becomes empty before the first rule applies, a deterministic function of the current state should be used to determine the next tank that goes in service. We point out one possible rule:

Serve the next tank in cyclic order.

For the switched arrival system the policy is inverse: A tank will be instantaneously served if it becomes empty, and the second switching rule has to be applied if a tank is full before another becomes empty.

If more than one tank is empty or filled in the same moment, we require that the system stops. These events correspond either to a mapping on unstable periodic orbits in the Poincaré maps (Sec.4.2.1 and Sec.4.3) or to discontinuity points of the Poincaré map. If the stop points are critical points (due to the discontinuity) their basin of attraction consists only of the points itself and is therefore of zero measure. If they are unstable fixed points their basin of attraction contains all their preimages. This set is of zero measure with respect to the Lebesgue measure, but can form a dense set. These orbits are, in other words, not typical for the considered hybrid dynamical systems.

If the total inflow into the switched tank systems is smaller or greater than the total outflow the systems will either empty in a finite time, or the total content of the systems grows (up to infinity if there are no upper thresholds) until all tanks reach their upper thresholds simultaneously. In both cases Zeno trajectories occur.

Hence we investigate the special (balanced) dynamical regime, where the total inflow into the system meets the total outflow. To avoid overflow or complete draining of all tanks we require that a balance condition is fullfilled. 
The balance condition for the switched server system is that $\sum_{i} \lambda_{i}=\Upsilon$ and for the switched arrival system that $\sum_{i} \mu_{i}=\Lambda$. Furthermore, the balance condition implies (here) that the total content of the system is constant (fixed by the initial conditions). Without loss of generality we normalize therefore $\Upsilon=1=\sum_{i} \lambda_{i}$ and $\Lambda=1=\sum_{i} \mu_{i}$ and $\sum_{i} x_{i}=1$. For simplicity we choose here the same maximum capacity $b$ for all tanks, $b_{i}=b$. As long as the system stays in the discrete state $q$ (i.e., the server is attached to tank q) the equation of motion for $x$ is simply:

$$
\dot{x}=v_{q}
$$

where $v_{q}=\lambda-e_{q}$ for the switched server system, and $v_{q}=e_{q}-\mu$ for the switched arrival system. Here $x=\left(x_{1}, x_{2}, \ldots, x_{n}\right)$ is the continuous state vector, $\left\{v_{q} \mid q=1, \ldots, n\right\}$ is the set of velocity vectors, $\lambda=\left(\lambda_{1}, \lambda_{2}, \ldots, \lambda_{n}\right), \mu=\left(\mu_{1}, \mu_{2}, \ldots, \mu_{n}\right)$ are constant vectors and $e_{q}$ is the $q$ 'th canonical unit vector in $\mathbb{R}^{n}$.

a)

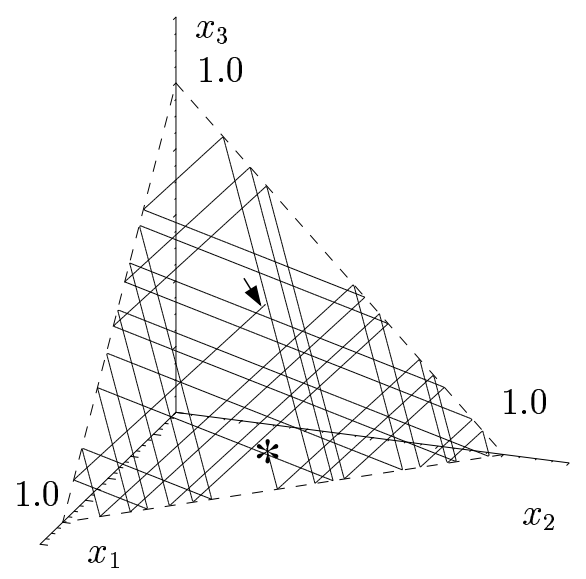

b)
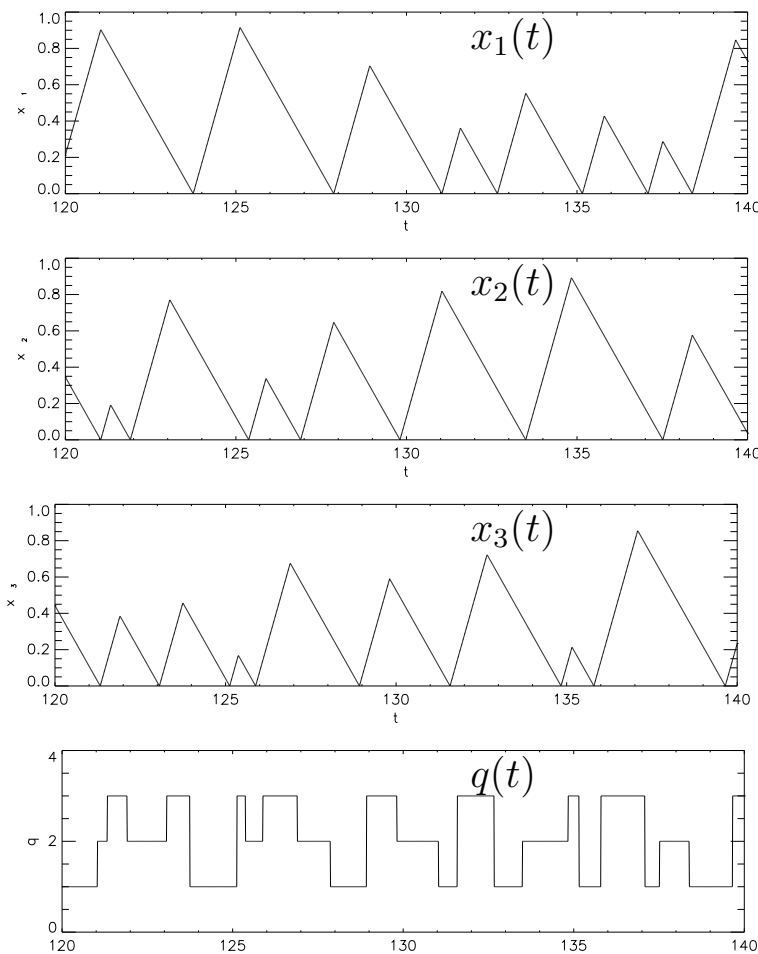

Figure 4.2: Sample trajectory of a three tank switched arrival system with $\lambda_{1,2,3}=1 / 3$ and $b=1$.(a) depicts a trajectory in the continuous part of the state space. The starting point is marked by ( $\searrow$ ) and the end with $(*)$. Samples of the time series of $x_{i}(t)$ and $q(t)$ are given in $\mathrm{b}$ ). 


\subsubsection{Strange Billiards and their Poincaré Maps}

The evolution of the system inside $\mathbb{R}^{n}$ reveals the structure of a strange billiard (see Fig.4.2). The current state moves uniformly and linearly inside the bounded region $S_{n}^{*}(b)=\left\{x \in \mathbb{R}^{n} \mid \sum_{i} x_{i}=1,0 \leq x_{i} \leq b ;\right.$ for $\left.i=1, \ldots, n\right\}$. The evolution changes according to the switching rules if the continuous state $x$ hits the boundary $\delta S_{n}^{*}(b)$ of $S_{n}^{*}(b)$. At this time instant $t_{m}$ the full state of the system changes $\left[x\left(t_{m}\right), q\left(t_{m}\right)\right] \mapsto\left[x\left(t_{m}\right), q\left(t_{m}^{+}\right)\right]$and a new velocity vector is selected from the set $\left\{\left(v_{q}\right) \mid q=1, \ldots, n\right\}$.

This can be interpreted as a strange reflection at the boundary. The balance condition implies that the allowed $v_{q}$ span a hyperplane of $\mathbb{R}^{n}$.

But in contrast to ordinary physical billiards, which are invertible Hamiltonian systems, these systems are not invertible. Furthermore, the angle of incidence in general does not equal the angle of reflection.

As ordinary billiards the systems can be studied by means of Poincaré mappings of the boundary onto itself. These Poincare mappings sample, in other words, the systems continuous state at the switching times.

Consider the Poincaré map $G: \delta S_{n}^{*} \rightarrow \delta S_{n}^{*}$. For the switched arrival as well as for the switched server system two successive hits of the boundary (at $t_{m}$ and $t_{m+1}$ ) are determined uniquely by $x\left(t_{m}\right)$ and we can formally write

$$
x\left(t_{m+1}\right)=G\left(x\left(t_{m}\right)\right)=x\left(t_{m}\right)+v_{q} \Delta t_{m}
$$

where $\Delta t_{m}=t_{m+1}-t_{m}$ is $\Delta t_{m}=\min \left(\left[x_{q}\left(t_{m}\right) /\left(1-\lambda_{q}\right)\right] ;\left[\left(b-x_{i}\left(t_{m}\right)\right) / \lambda_{i}\right]_{i \neq q}\right)$ for the switched server system and $\Delta t_{m}=\min \left(\left[\left(b-x_{q}\left(t_{m}\right)\right) /\left(1-\mu_{q}\right)\right] ;\left[x_{i}\left(t_{m}\right) / \mu_{i}\right]_{i \neq q}\right)$ for the switched arrival system.

\subsubsection{Limiting Cases}

For the normalized switched systems (with $n>2$ ) two limit cases with respect to the shape of $S_{n}^{*}$ exist. For $b=1, S_{n}^{*}$ is the usual $n$-simplex embedded in $\mathbb{R}^{n}$ given as $\bar{S}_{n}=S_{n}^{*}(b=1)=\left\{x \in \mathbb{R}^{n} \mid \sum_{i} x_{i}=1,0 \leq x_{i} \leq 1\right.$; for $\left.i=1, \ldots, n\right\}$. If $b=1 /(n-1), S_{n}^{*}$ is a geometrically similar regular $n$-simplex which is now smaller and inverted, given as $\tilde{S}_{n}=S_{n}^{*}(b=1 /(n-1))=\left\{x \in \mathbb{R}^{n} \mid \sum_{i} x_{i}=1,0 \leq x_{i} \leq\right.$ $1 /(n-1)$; for $i=1, \ldots, n\}$. Figure 4.3 shows an illustration of these two limit cases for $n=3$.

Now we shall examine the properties of $G$ on the boundaries $\delta \bar{S}_{n}$ and $\delta \tilde{S}_{n}$. For the switched server system on $\bar{S}_{n}$ it was shown [25] that G is everywhere contracting. In contrast, for the switched arrival system on $\bar{S}_{n}$ the Poincare map $G$ is chaotic and the invariant measure can be derived by constructing the Frobenius-Perron operator. The 
piecewise constant probability measure invariant under $\mathrm{G}$ is given by

$$
p^{*}\left(\bar{s}_{x_{i}=0, n}\right)=\frac{1}{d} \mu_{i}\left(1-\mu_{i}\right)
$$

with $d=\sum_{i} \mu_{i}\left(1-\mu_{i}\right)$ and $\bar{s}_{x_{i}=0, n}=\left\{x \in \mathbb{R}^{n} \mid x_{i}=0, x \in \bar{S}_{n}\right\}$ denotes the i'th face of $\bar{S}_{n}\left(\delta \bar{S}_{n}=\bigcup_{i=1}^{n} \bar{s}_{x_{i}=0, n}\right)$ [92]. Moreover, the generating partition of the Poincaré map is given by sub-simplexes exactly corresponding to the discrete states of the hybrid system.For $\tilde{S}_{n}$ choose the transformations

$$
\tilde{e}_{q}=\frac{1}{n-1}\left(\mathbb{I}-e_{q}\right)
$$

and

$$
\begin{aligned}
& \tilde{\mu}=(n-1)^{-1}(\mathbb{I}-\lambda) \\
& \tilde{\lambda}=(n-1)^{-1}(\mathbb{I}-\mu)
\end{aligned}
$$

with $\mathbb{I}=\sum_{i=1}^{n} e_{i}$. This constitutes a complete orthonormal system (with mirrored handedness) where the equation of motion and the Poincare map $G$ for the switched server system on $\tilde{S}_{n}$ are (up to the scaling factor $1 /(n-1)$ ) just the ones of the switched arrival system on $\bar{S}_{n}$ and vice versa.

For $b<1 /(n-1)$ the system dynamics is the same as for $b=1 /(n-1)$, restricted to a smaller and smaller n-simplex up to $b=1 / n$ where the simplex vanishes.

a)

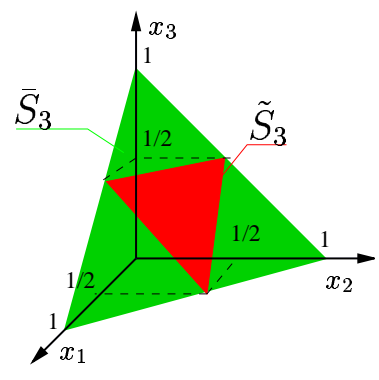

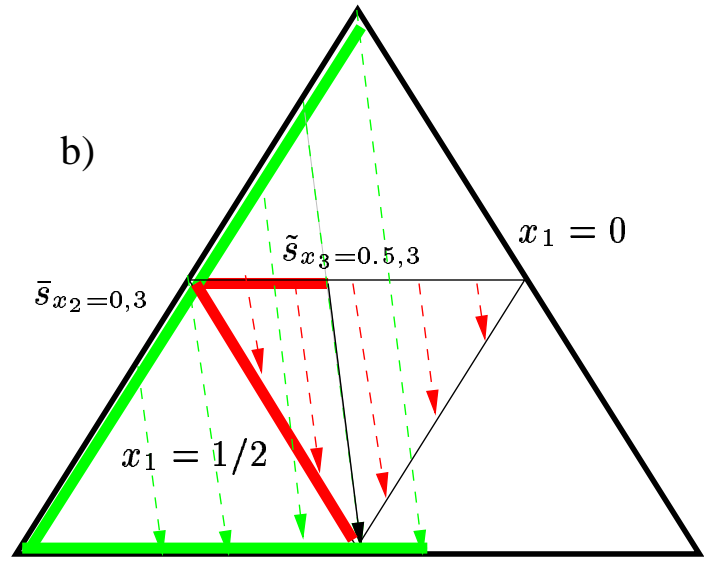

Figure 4.3: Illustration of the continuous part of the state space embedded in $\mathbb{R}^{3}$. (a) The two 3simplexes $\bar{S}_{3}$ (green) and $\tilde{S}_{3}$ (red) that correspond to the limiting cases $b=1$ and $b=1 / 2$, respectively. (b) Contracting and expanding properties of one and the same velocity vector inside $\bar{S}_{3}$ and $\tilde{S}_{3}$, respectively. The green edge of $\bar{S}_{3}$ (corresponding to $\bar{s}_{x_{2}=0,3}$ ) is mapped under $v_{3}$ to a shorter segment at the bottom of $\bar{S}_{3}$, whereas the red segment of the top edge of $\tilde{S}_{3}$ (subset of $\tilde{s}_{x_{3}=1 / 2,3}$ ) is expanded by the same flow. 


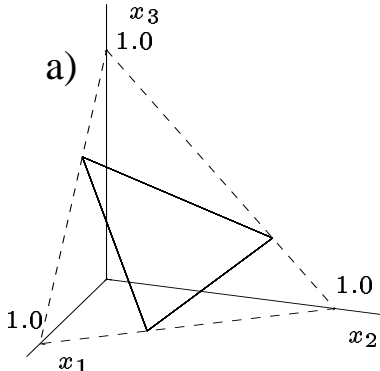

d)

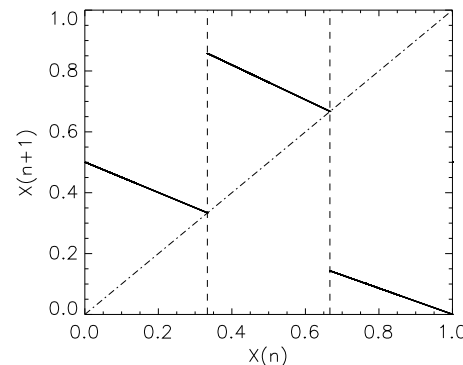

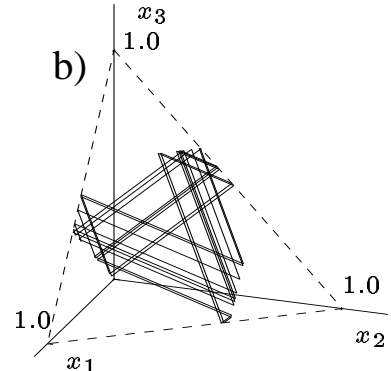

e)

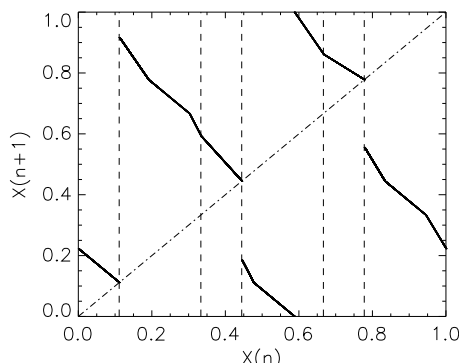

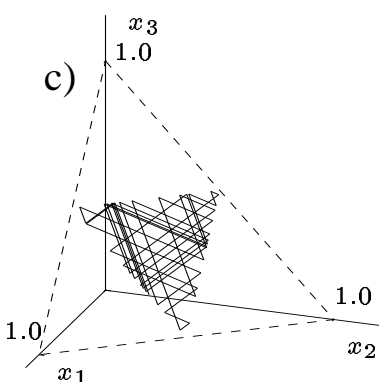

f)

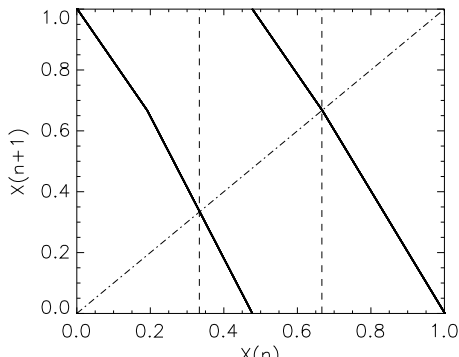

Figure 4.4: Sample trajectories and Poincaré maps of a three tank switched server model, filling rates $\lambda_{1}=0.3, \lambda_{2}=0.4, \lambda_{3}=0.3$. The upper threshold is varied: The first row shows trajectories for a) $b=1.0$, b) $b=0.62$ and c) $b=0.5$.

The lower row depicts the Poincare maps of the system. The main segments (corresponding to faces of $\left.S_{3}^{*}\right)$ are indicated by dashed lines. $X(n)=X(n+1)$ is shown by a dot-dashed line. a) Everywhere contracting Poincaré map with 2 discontinuities for $b=1.0$ where only switchings at the lower threshold take place. b) $b=0.7$, c) $b=0.6$ d) Bernoulli type Poincaré map for $b=0.5$ where only switchings at the upper threshold take place.

\subsection{Dynamics and Bifurcations}

The dynamics of strange billiards depends besides the configuration and the $\lambda$ and $\mu$ parameters also on the shape of the boundary, determined by $b$. In the following we shall discuss the dynamics with respect to $b$ as bifurcation parameter. We restrict our analysis to $n=3$ and consider the switched server system. Applying the results of Sec.4.2.2 the transfer to the switched arrival system is straightforward.

\subsubsection{Properties of Poincaré Maps}

For three tanks the dynamics is restricted to $S_{3}^{*}$ which lies on a two dimensional manifold, and is generally a 6-simplex. The boundary $\delta S_{3}^{*}$ is one dimensional. After suitable changes of coordinates $\left(T: \delta S_{3}^{*} \rightarrow[0,1] ; x \mapsto X\right)$ and rescaling of the parameters the resulting Poincaré maps are piecewise linear maps of the unit interval 
onto itself (Fig. 4.4). This normalized Poincaré maps are given in analytical form in table A.1(see Appendix A). By definition all branches of the Poincaré map are rightcontinuous and $q$ is a function of $X$.

For $b=1$ (where $S_{3}^{*}=\bar{S}_{3}$ is a triangle (see Fig. 4.3a) we obtain an everywhere contracting piecewise linear map with three different branches and three discontinuity points (Fig. 4.4d). For $b=1 / 2$ a Bernoulli type map with six segments of constant slope and two discontinuities arises (Fig. 4.4f). The branches are given by the transformed coordinates of the vertices of $\tilde{S}_{3}$ and their pre-images on $\delta \tilde{S}_{3}$. For the switched server systems in between the limit cases $b=1$ and $b=1 / 2$ we obtain a parameter dependent morphing between an everywhere contracting map and a Bernoulli type map (Fig. 4.4e). The mechanism, typical for strange billiards, is the expansion of linear branches and the creation of new branches of the map with decreasing $b$.

In general, the maps have 6 main segments, associated with the 6 faces of $S_{3}^{*}$, denoted as $s_{x_{1}=0}, s_{x_{3}=b}, s_{x_{2}=0}, s_{x_{1}=b}, s_{x_{3}=0}, s_{x_{2}=b}$ in this sequence. Each of the 6 segments possesses a number of sub segments $g$ (up to three sub segments for $s_{x_{i}=b}$ and up to two for $s_{x_{i}=0}$ ), where the slope of the Poincaré map is constant. Whereas the exact slope ( $a(g)$ with $a<0$ everywhere) is defined by $\left\{\lambda_{i} \mid i=1,2,3\right\}$, some general properties depend only on the main segments (faces of $S_{3}^{*}$ ) to which the sub segment maps. We denote the possibilities for the segments: A segment belonging to $g\left(s_{x_{i}=b} \mapsto s_{x_{i}=0}\right)$ or $g\left(s_{x_{i}=0} \mapsto s_{x_{i}=b}\right)$ has $a=-1$, providing interval exchanges. A segment $g\left(s_{x_{i}=b} \mapsto s_{x_{j}=b}\right)$ has $a<-1$ and segments $g\left(s_{x_{i}=0} \mapsto s_{x_{j}=0}\right)$ contribute contracting branches $(a>-1)$. For segments $g\left(s_{x_{i}=0} \mapsto s_{x_{j}=b}\right)$ the slope is given by $a=-\left(\lambda_{i} / \lambda_{j}\right)$ and the associated branches are contracting, neutrally or expanding depending on $\left\{\lambda_{i}\right\}$. Thus, for the strange billiard expanding properties are closely related to faces where $x_{i}=b$.

The discontinuities of the map are given by the vertices $s_{x_{1}=0} / s_{x_{3}=b}, s_{x_{2}=0} / s_{x_{1}=b}$ and $s_{x_{3}=0} / s_{x_{2}=b}$. By construction a further discontinuity at the pre-image of the point where we sliced $\delta S_{3}^{*}$, (i.e. the vertex $s_{x_{2}=b} / s_{x_{1}=0}$ and $s_{x_{2}=b} / s_{x_{3}=b}$ for $b=0.5$ ) is obtained. It is obvious that not all branches are present for all $b$. Moreover, the properties of some branches depend on $\left\{\lambda_{i}\right\}$. Therefore different types of maps, connected with different admissible orbits specify the dynamics for different $b$. This is crucial for the occurrence of chaotic behavior. A chaotic attractor is governed by more local expansion than contraction during its evolution. Therefore chaotic behavior is only possible if branches with expanding properties exist and are visited sufficiently often. A simple example for the creation of expanding branches and the associated change of the dynamics is depicted in Fig. 4.5a. Here with $\lambda_{i}=1 / 3$ the only expanding segments $g\left(s_{x_{i}=b} \mapsto s_{x_{j}=b}\right)$ appear simultaneously for $b<2 / 3$ (which can be derived from simple geometrical arguments) and in this parameter region also chaotic behavior is obtained. 
a)
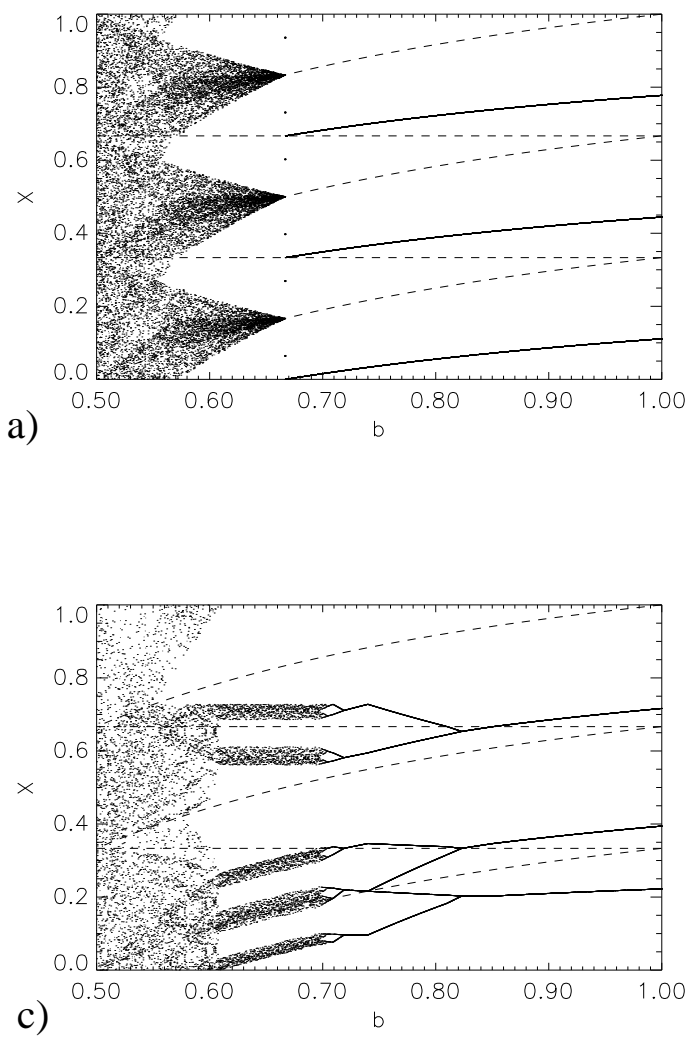

d)
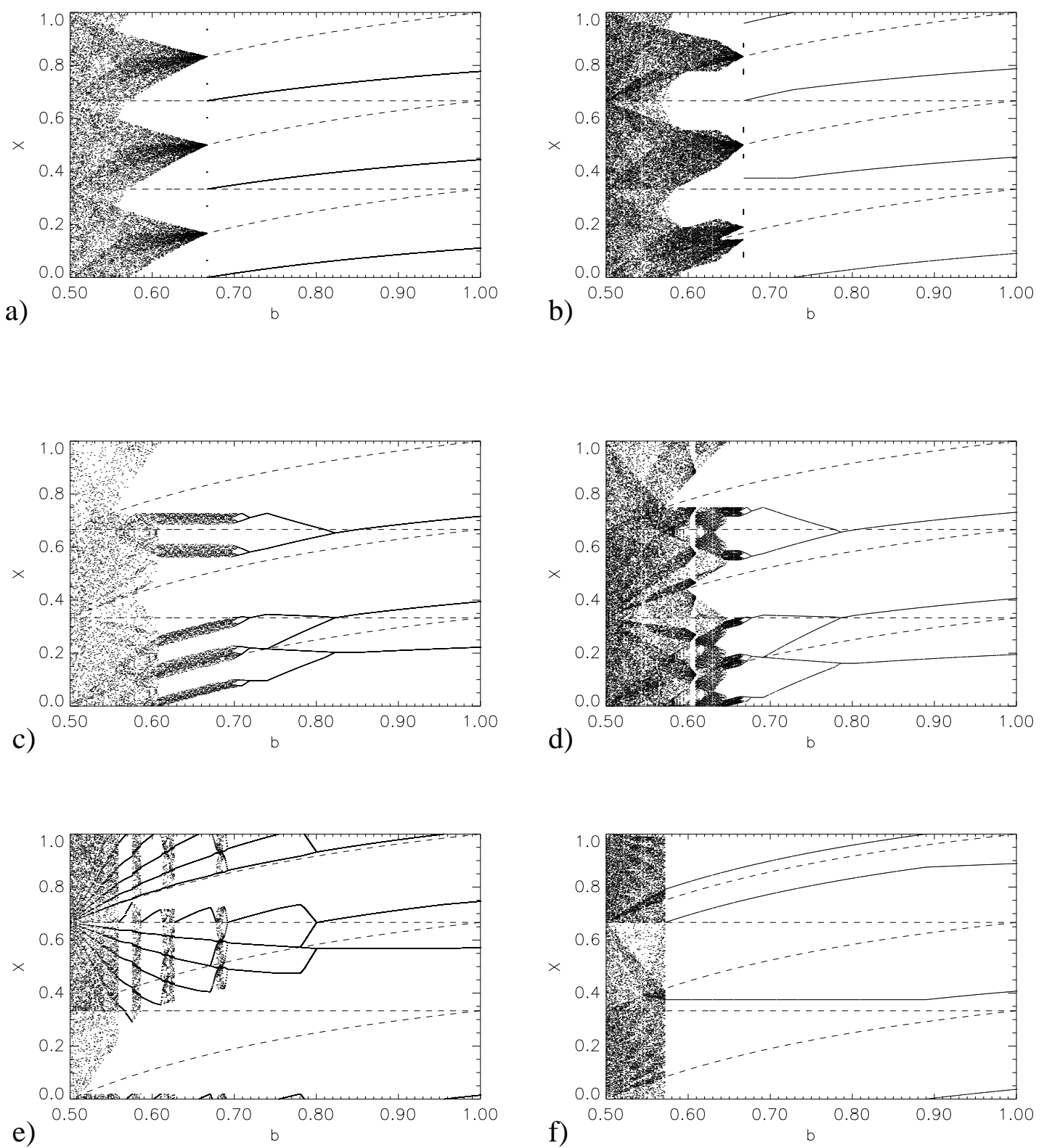

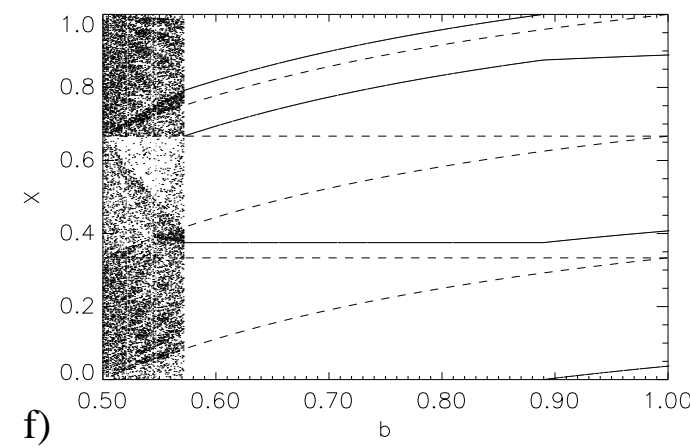

f)

Figure 4.5: Bifurcation diagrams for three tank switched server models showing the normalized boundary coordinate $X$ vs. maximum tank capacity $b$. The main segments are indicated by dashed lines.

a) filling rates $\lambda_{1}=\lambda_{2}=\lambda_{3}=1 / 3$.
b) filling rates $\quad \lambda_{1}=0.3, \quad \lambda_{2}=0.4, \quad \lambda_{3}=0.3$.
c) filling rates $\lambda_{1}=0.49, \quad \lambda_{2}=0.11, \quad \lambda_{3}=0.4$.
d) filling rates $\lambda_{1}=0.45, \quad \lambda_{2}=0.15, \quad \lambda_{3}=0.4$.
e) filling rates $\lambda_{1}=0.15, \quad \lambda_{2}=0.8, \quad \lambda_{3}=0.05$
f) filling rates $\quad \lambda_{1}=0.1, \quad \lambda_{2}=0.6, \quad \lambda_{3}=0.3$.

Note, that all bifurcations in this diagrams are border collision bifurcations, occuring from collisions of periodic points with borders of subsegments of the Poincaré maps. 


\subsubsection{Bifurcations in three tank switched server systems}

In a parameter region where neither new segments appear nor existing segments vanish, the slope and (dis-) continuity of the branches are invariant for changing $b$ but their end points and extension are altered. In this case a bifurcation can occur, if a point of a periodic orbit of the map hits the border of a segment. At this border the map is either continuous or has a discontinuity. In the billiard picture the hit of a segment border corresponds to a reflection point at the boundary, that hits a vertex. In view of the different numbers of segments for different $b$, the number of critical points in the maps and the fact that the segments are relatively small, it is clear that the entire structure of the dynamics of strange billiards can be quite complicated. Figure 4.5 provides some examples of bifurcation diagrams. For some areas in the parameter space orbits with marginal stability may occur due to the fact that expansion and contraction for the orbit cancel each another. This typically requires a certain symmetry for the velocity vectors $v_{q}$.

In general coexisting attractors touching different subsets of $\delta S_{3}^{*}$ are possible.

If a periodic point moves from one segment (slope $a_{L}$ ) on a continuous branch to another with slope $a_{R}$ border collision bifurcations of continuous piecewise linear maps (see Chap.3) can occur. Because all slopes in the Poincaré map are smaller than zero, either both slopes of the segments (in an n-fold Poincaré map for a periodic orbit) are greater than zero (even period) or smaller than zero (odd period). Therefore, as discussed in Sec.3.2, three cases of border-collision bifurcations of continuous maps for periodic orbits can occur here:

1. If $\left|a_{L}\right|<1$ and $\left|a_{R}\right|>1$ a period doubling bifurcation takes place if 3 . does not apply.

2. If $\left|a_{L}\right|<1$ and $\left|a_{R}\right|<1$ there is a unique attractor on both segments, only the path of the fixed point changes.

3. If both slopes are smaller than zero $\left(a_{L}>-1\right.$ and $\left.a_{R}<-1\right)$ and $1-a_{R} a_{L}<0$ the periodic attractor vanishes.

An example of a border collision bifurcation is depicted in Fig. 4.6. For decreasing $b$ a period-3 orbit exists up to the moment where the face of $x_{1}=b$ hits the periodic point on $s_{x_{3}=0}$ ( $\uparrow$ in Fig. 4.6a,c). There the fixed point in the three-fold Poincare map (Fig. 4.6b) crosses from the segment with slope $(-1<a<0)$ to the segment with slope $(-2>a)$ and a period- 6 attractor is born. With decreasing $b$ the reflection points of this attractor move along $\delta S_{3}^{*}$ as shown in Fig. 4.6c. The next border crossing occurs 
a)
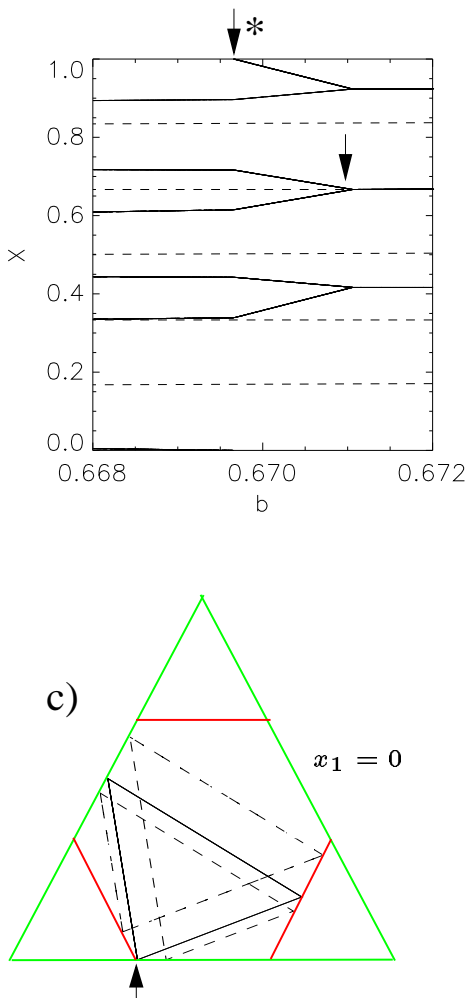

b)
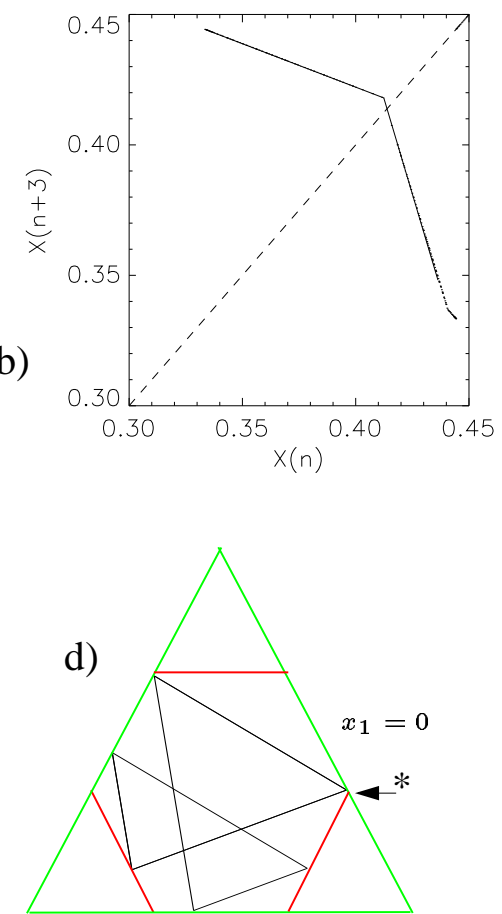

Figure 4.6: a) Bifurcation diagram for a three tank switched server model with filling rates $\lambda_{1}=0.26$, $\lambda_{2}=0.49, \lambda_{3}=0.25$. b) Part of the three-fold Poincaré map in the section $x_{2}=0$ at $\left.b=0.670, \mathrm{c}\right)$ The period- 3 orbit (solid line) at $b=0.672$ before the border collision, and the period- 6 orbit (dashed line) at $b=0.670$ after the bifurcation, d) The modified period 6 orbit at $b=0.668$ after border crossing

when the reflection point of the period- 6 attractor crosses the vertex $s_{x_{2}=b} / s_{x_{1}=0}(\uparrow *$ in Fig. 4.6a,d) but no bifurcation takes place $\left(0<a_{L, R}<1\right.$ in the 6-fold Poincaré map) only the path with respect to $b$ is altered. A second border collision bifurcation (not depicted in Fig. 4.6) will follow when a reflection point of the orbit hits the vertex $s_{x_{2}=0} / s_{x_{3}=b}$. As the example shows it is typical for strange billiards that bifurcations occur on different branches in a rapid sequence if the orbit contains reflection points on faces with $x_{i}=b$.

Whereas the role of border collision bifurcations of continuous maps in the entire scenario is limited, the discontinuities are in some sense essential for the dynamics of the systems. When due to varying $b$ a periodic point hits a point of discontinuity, it is mapped to a completely distinct site. It is well known that critical points caused by a discontinuity can induce a rich variety of dynamical behavior in maps on the unit interval.This includes period adding scenarios and unusual transitions to chaos as al- 
a)

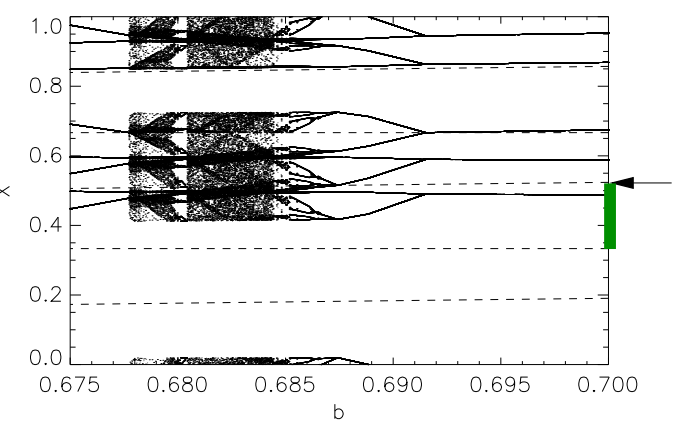

b)
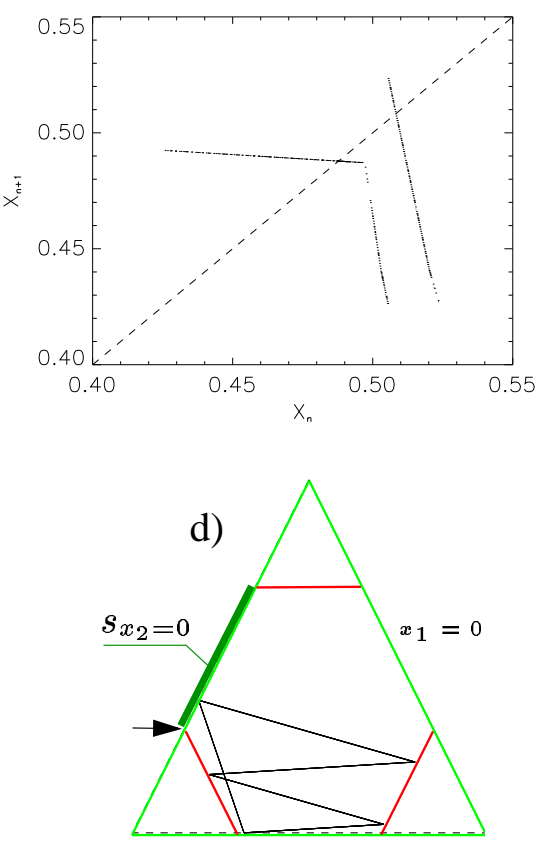

c)
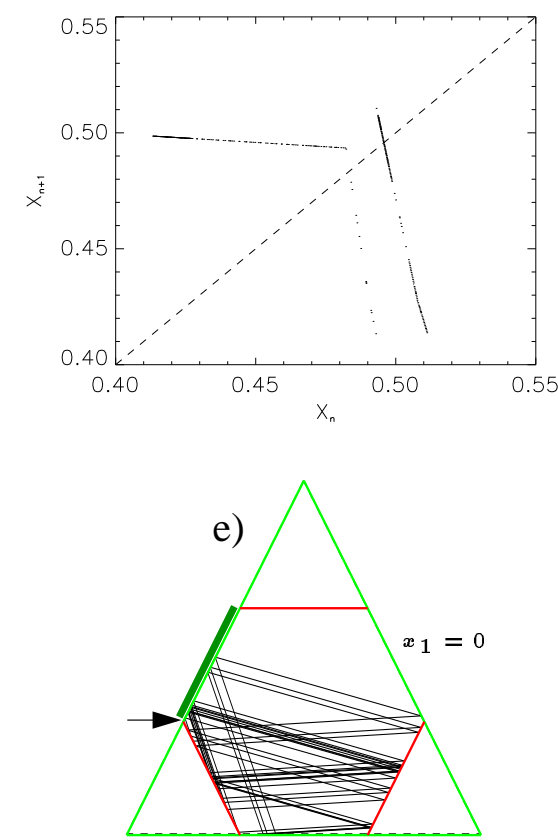

Figure 4.7: a) Bifurcation diagram for a three tank switched server model with filling rates $\lambda_{1}=0.15$, $\lambda_{2}=0.8, \lambda_{3}=0.05$ (compare Fig. $4.5 \mathrm{c}$ ) b) Reduced return map for $s_{x_{2}=0}$ at $b=0.70$. c) Reduced return map for $s_{x_{2}=0}$ at $b=0.682$. d) Stable period-5 orbit for $b=0.7$ given by the stable fixed point at the first segment of the reduced return map. e) Sample trajectory from the chaotic attractor at $b=0.682$.

ready discussed in Sec.3.3.

For a detailed investigation it is often useful to consider return maps for a subset of $X$, preferably of a main segment or face of the 6-simplex onto itself instead of the full Poincaré map. Such reduced return maps contain a number of segments, typically associated with topologically different paths which map (usually within different numbers of iterations) points located on the choosen subset back to this subset.

Figure 4.7 gives an example of a bifurcation scenario involving a discontinuity. The 

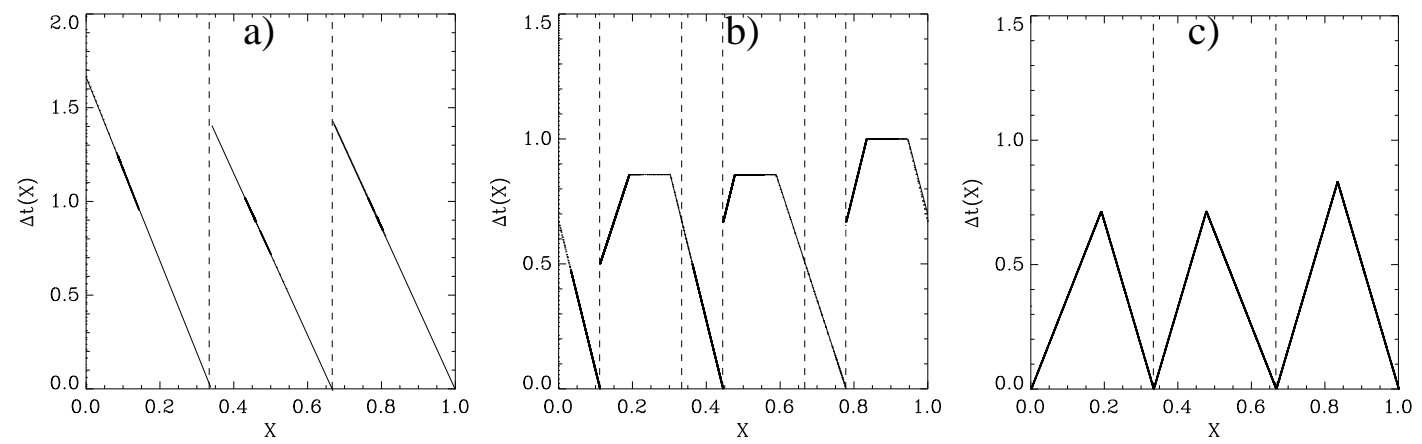

Figure 4.8: Examples of the function $M: X \mapsto \Delta t$ for a three tank switched server system, filling rates $\lambda_{1}=0.3, \lambda_{2}=0.4$ (cf. Fig.4.5). The upper threshold is varied: a) $b=1.0$, b) $b=0.6$ and c) $b=0.5$. The main segments of $X$ (corresponding to faces of $S_{3}^{*}$ ) are indicated by dashed lines.

only relevant discontinuity for the considered family of attractors is the one located at the vertex $s_{x_{2}=0} / s_{x_{1}=b}$ (marked by $\uparrow$ in Fig. $4.7 \mathrm{a}, \mathrm{d}, \mathrm{e}$ ). We obtain the reduced return map $H$ for the face $s_{x_{2}=0}$ (Fig. 4.7b,c). Here, the discontinuity in the reduced return map for $s_{x_{2}=0}$ separates branches where the orbits return to $s_{x_{2}=0}$ within the fifths iteration and those where the orbit returns within the third iteration. For $b=0.7$ a stable period-5 orbit is obtained, indicated by a stable fixed point on the leftmost segment of the reduced return map (Fig. 4.7b,d). After border collision bifurcations for decreasing $b$ all segments of $H$ become involved in the attractor formation.

\subsubsection{Dynamics of Switching Times}

To describe the full dynamics of the hybrid system not only the filling levels $(x)$ of the tanks or their transformed equivalents $X$ (given by iteration of the Poincaré map) at the switching times are required but also the switching times $t_{m}$ or the intervals $\Delta t_{m}$. The interval $\Delta t_{m}$ for given rates and tank capacities in a switched server system is uniquely determined by $x\left(t_{m}\right)$ or $X\left(t_{m}\right)$ and is given by a piecewise linear function $M:[0,1] \rightarrow \mathbb{R}, X(t) \mapsto \Delta t$. Some typical examples of this function are depicted in Fig.4.8 for different thresholds $b$. The function $M=M(X, \lambda, b)$ has segments located at the same locations as these of $G(X, \lambda, b): X\left(t_{m}\right) \mapsto X\left(t_{m+1}\right)$ since the time to the next switching (i.e. the next hit of the boundary $\delta S^{*}$ ) is proportional to the distance of the next boundary segment in the direction of the determined velocity vector. The function $M(X)$ can be determined analytically. The segments of $M(X)$ are of constant slope, determined by $\left\{\lambda_{i}\right\}$ and for segments $m\left(s_{x_{i}=b} \mapsto s_{x_{i}=0}\right)$ and $m\left(s_{x_{i}=0} \mapsto s_{x_{i}=b}\right) M(X)=$ const. The function $M(X)$ is not invertible.

Moreover, a map that considers $\Delta t_{m} \mapsto \Delta t_{m+1}$ is in general not unique (see Fig.4.9 for an example). Only for systems where all (fill) rates are equal and either $b=1$ or $b=1 / 2$ this map is unique and allows a study of the systems dynamics directly from successive inter-switching time intervals $\Delta t_{m}$. 

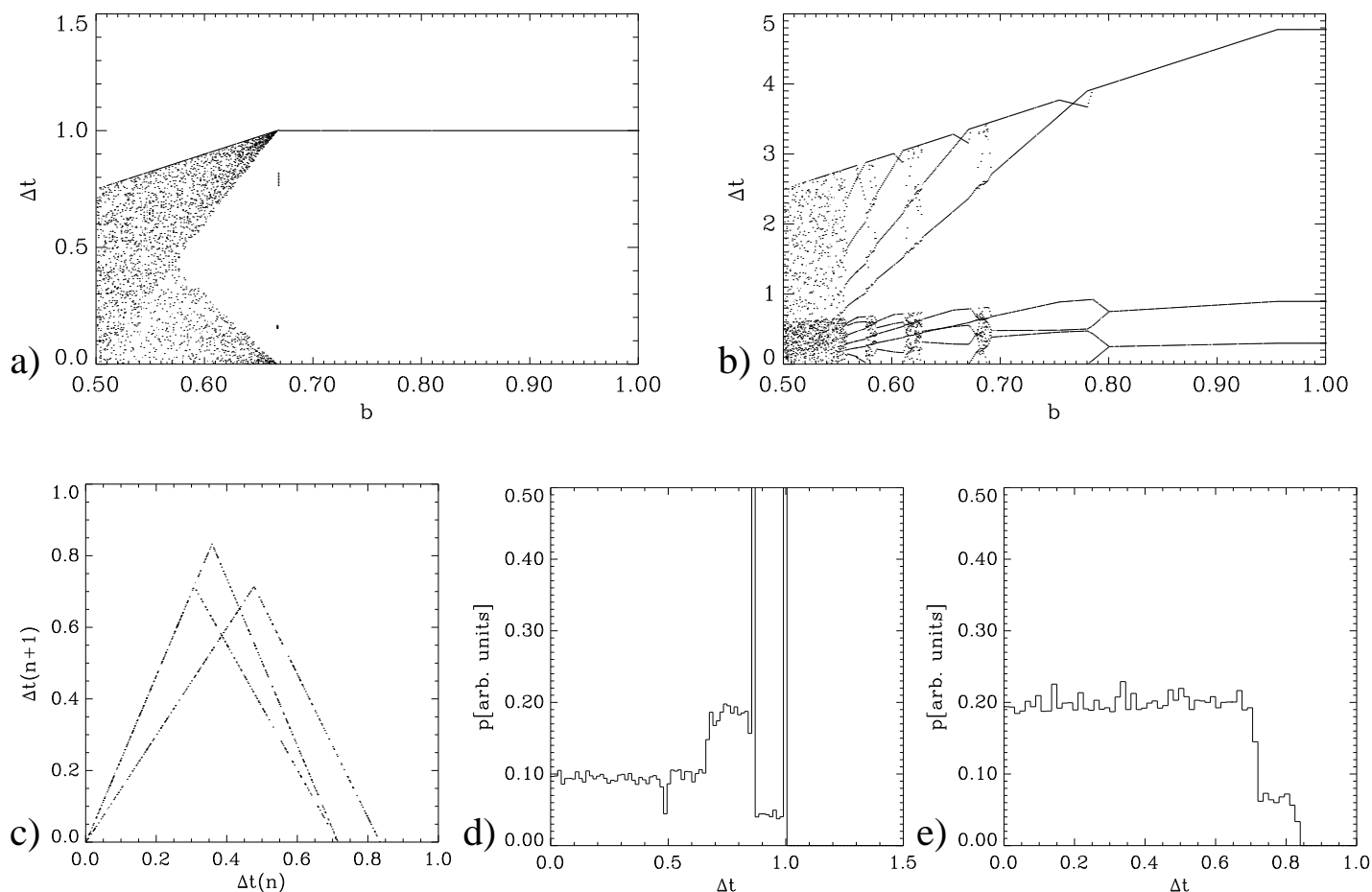

Figure 4.9: Examples of bifurcation diagrams and numerically estimated distributions of interswitching time intervals $\Delta t$. The bifurcation diagrams of $\Delta t$ vs. the maximum tank capacity $b$ for three tank switched server systems in the first row are computed for a): $\lambda_{1,2,3}=1 / 3$ and b) $\lambda_{1}=0.15$, $\lambda_{2}=0.8, \lambda_{3}=0.05$ (cf. Fig.4.5a,e). c) Example for a map $\Delta t_{m+1}$ vs. $\Delta t_{m}$ (computed for $\lambda_{1}=\lambda_{3}=0.3, \lambda_{2}=0.4$ and $\left.b=1 / 2\right) \mathrm{d}$ ),e) histograms of inter-switching times for a three tank switched server system with the parameters a). d) $b=0.6$, e) $b=1 / 2$.

However, a bifurcation diagram of $t$ vs. the maximum tank capacity contains similar information on the system dynamics as a bifurcation diagram of normalized boundary coordinates with two major drawbacks (c.f. Fig.4.9a,b and Fig.4.5a,e). Firstly, the period of $\Delta t$ allows no conclusion on the period of the hybrid system since tanks that obtain equal (filling) rates produce switching time intervals that are equal and do not distinguish the different tanks. Secondly, whole boundary segments of the simplex in the countinuous part of the state space (those that map $m\left(s_{x_{i}=b} \mapsto s_{x_{i}=0}\right)$ ) lead to only one value for $\Delta t_{m}$.

If we consider the series of switching events as deterministic point process, the invariant probability density of inter-switching time intervals $p(\Delta t)$ is of interest. This probability density can be obtained by $p(\Delta t)=M(X) p(X)$ if the invariant probability density $p(X)$ of the Poincare map is known. For periodic orbits of the hybrid system $p\left(\Delta t_{m}\right)$ is a set of some $\delta$ peaks. For intermediate tank capacities, even if the hybrid system is chaotic, some inter-switching times will occur with a high probabil- 
ity due to the horizontal segments in $M(X)$ and generally in some intervals of $\Delta t$, $p\left(\Delta_{t}\right)$ will be zero. Only in the limiting case of $b=1 / 2$ where the hybrid system is chaotic and the Poincaré map has a piecewise constant invariant probability measure even $p\left(\Delta t_{m}\right)$ will be a piecewise constant function. Fig.4.9d,e provides two examples of numerically estimated histograms of $p(\Delta t)$.

\subsubsection{Complexity of Symbolic Dynamics}

For hybrid systems of the discussed type two types of symbolic dynamics can be considered. The first type is the usual symbolic dynamics of the Poincaré maps. Once a generating partition for the Poincaré map is found the symbolic dynamics is a useful tool for analytical studies. On the other hand we have the symbolic dynamics induced by the discrete states of the hybrid system. If we label different states of the hybrid system with symbols, the temporal evolution of the system produces a symbol sequence. Each segment $g$ of the Poincare map, or section of the faces of $\delta S_{3}^{*}$, is uniquely associated with a symbol that equals $q$ for the time interval until the next hit of the boundary in the hybrid system. In general this hybrid system related partition of the map is neither generic nor generating. Only for special cases, as for $b=1 / 2$ in the switched server system, both types of partitions are equivalent and the entropy of the symbol sequence of hybrid system states can be derived directly from the invariant probability measure of the Poincaré map.

\subsection{Further Models}

\subsubsection{Modified Switching Rules}

As we pointed out above, the switching rules for the switched server system and for the switched arrival system (at the lower and upper threshold, respectively) are not predetermined by system requirements. Two further possible choices for the switched server rule at the lower threshold are:

Serve the tank that needs the shortest time to become empty.

Serve the tank that needs the most time to become empty.

Apply rule 4.7 unless tank 3 is empty. In that case serve the tank that needs the shortest time to become empty.

All these rules imply, in contrast to the choice that was made in Sec.4.2 that every boundary section $s_{x_{i}=0}$ contains two distinct subsections, where a switching leadsto 

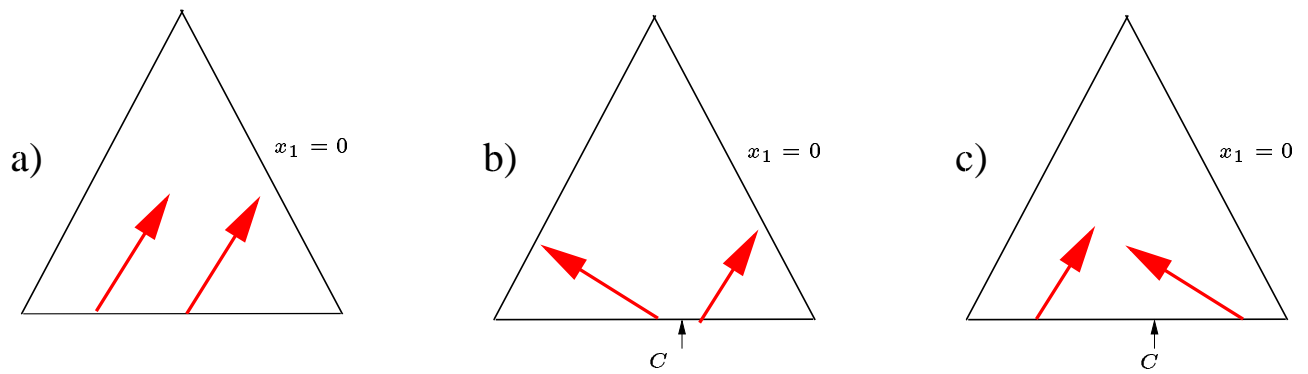

Figure 4.10: Scheme of the vector fields for different switching rules. a) Cyclic switching rule 4.2 b) Switching rule 4.6 and c) rule 4.8 .

a)

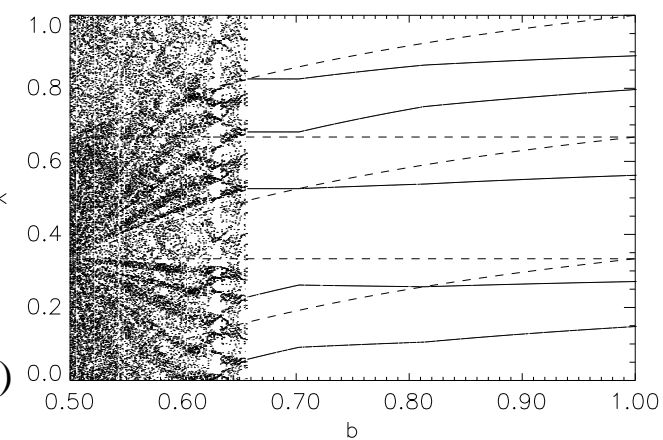

b)

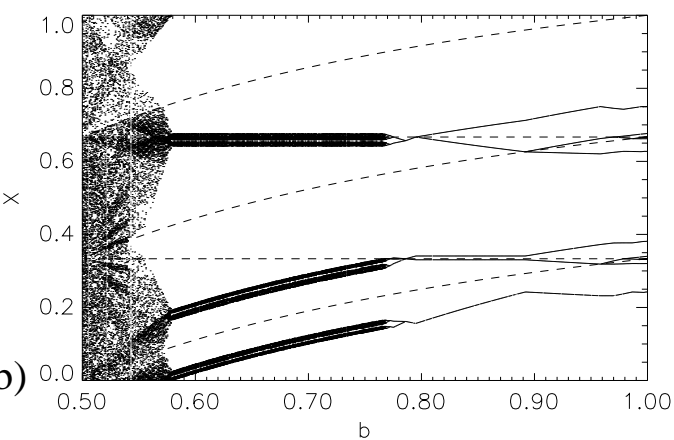

Figure 4.11: Bifurcation diagrams for three tank switched server models. The main segments are indicated by dashed lines a) Filling rates $\lambda_{1}=0.45, \lambda_{2}=0.15, \lambda_{3}=0.40$, switching rule 4.6 is applied b) Same filling rates as a) but switching rule 4.7 is applied. For the same system with rule 4.2 compare Fig.4.5d.

different discrete states $q\left(t_{m}^{+}\right)$and even to different velocity vectors. A sketch of the resulting velocity vectors is depicted in Fig.4.10. The point $C$, where the two subsections meet, is determined by $x_{j}(C)=\left(1-\lambda_{j}\right) /\left(2-\lambda_{j}-\lambda_{k}\right)$ at side $x_{i}=0$ in the full system where $x=\left(x_{i}, x_{j}, x_{k}\right), \lambda=\left(\lambda_{i}, \lambda_{j}, \lambda_{k}\right)$ and $x_{j}(C)=1-x_{k}(C)$.

The main impact of such modified switching rules is an increasing number of critical points (discontinuities) in the Poincaré map. This essentially changes the admissible orbits and makes coexisting orbits more likely. On the other hand the fundamental bifurcation mechanisms are not changed. Figure 4.12 shows two bifurcation diagrams that are computed with the modified switching rules 4.7 and 4.8 .

Figure $4.12 \mathrm{~b}$ provides an example where in a certain parameter region (around $b=0.67$ ) coexisting with a stable period-3 orbit another family of attractors undergoes a period adding scenario (see Sec. 3.3.2). 
a)
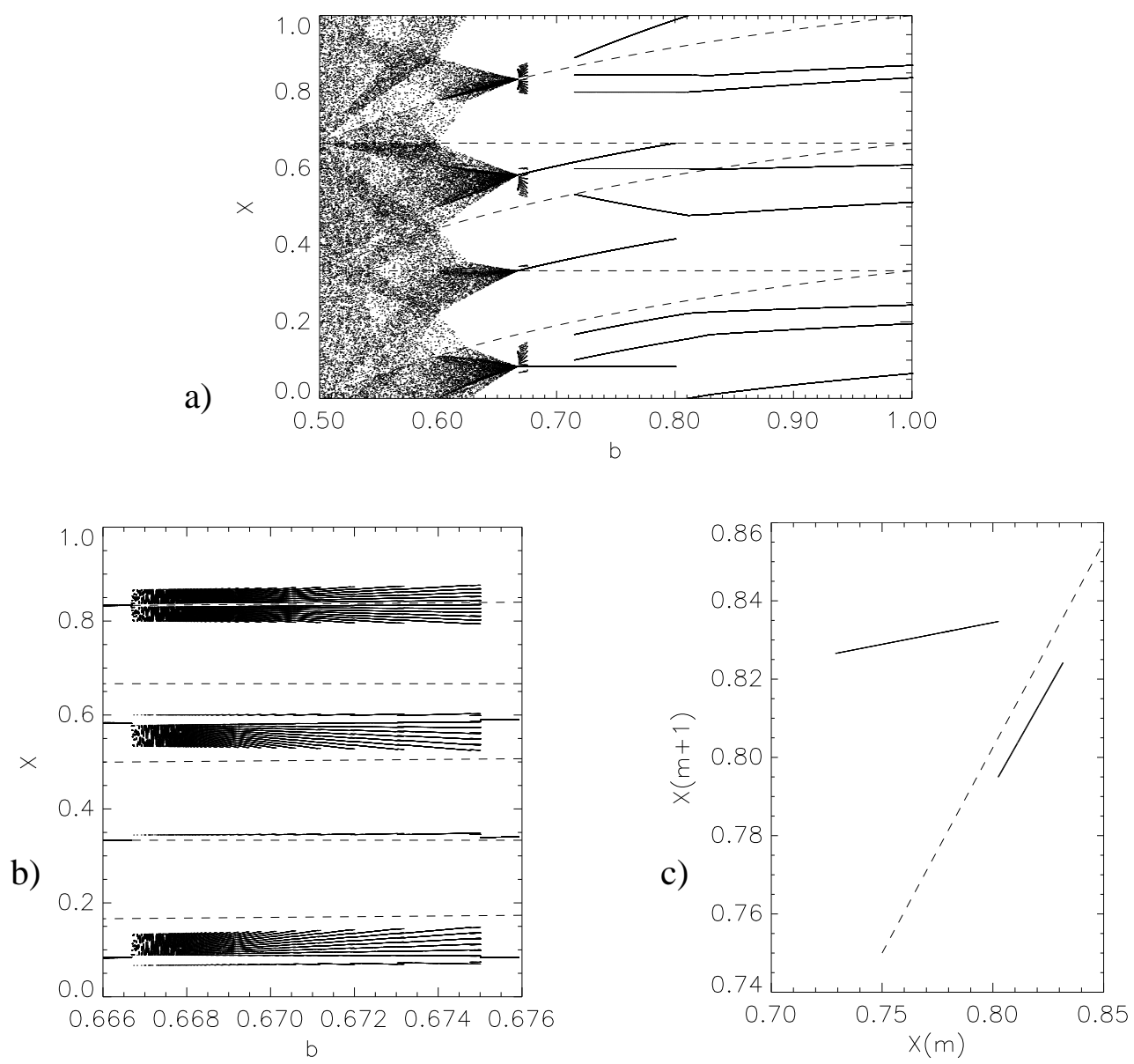

Figure 4.12: Example of a period adding scenario obtained for a switched server system. a) Bifurcation diagram. The main segments are indicated by dashed lines. Filling rates $\lambda_{1}=0.5, \lambda_{2}=0.25, \lambda_{3}=$ 0.25 . Switching rule 4.8 is applied. b) A section of a) showing a family of periodic attractors undergoing a sequence of period adding bifurcations. c) Reduced return map for $s_{x_{3}=0}$ at $b=0.674$, same parameters as b), showing the return map that provides the mechanism of the period adding scenario (see Sec.3.3.2).

\subsubsection{An Asymmetric Billiard}

This subsection provides an example of a three tank switched server system, where only to the first tank an upper threshold $b$ is assigned (Fig. 4.13). In this hybrid system different tanks are emptied with different priorities. Whereas the server stops instantaneously an emptying of tank two or three to serve tank one if its content reaches the threshold $b$, tank two is served only, if tank one was emptied in the foregoing time interval. Tank three finally is only served, if tank two was emptied previously and the content of tank one is below the threshold.

Now consider the dynamics of this asymmetric system. The balance condition is applied and all variables are normalized as in Sec.4.2. Every possible orbit has to strike 

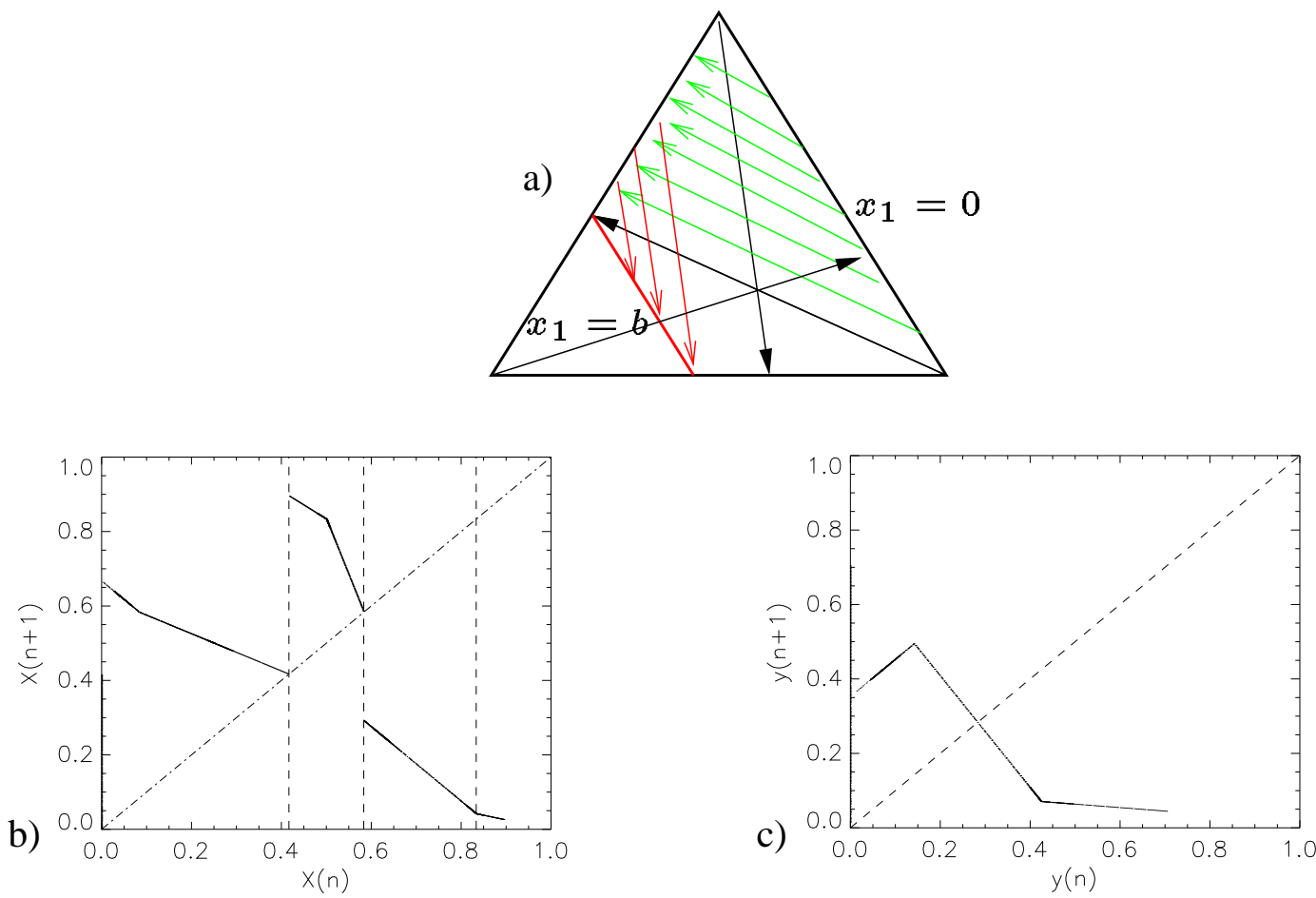

Figure 4.13: a) Illustration of the continuous part of the state space of the switched server system with an upper threshold assigned to the first tank only. b) Poincaré map $G$ of the system for $\lambda_{1}=0.2$, $\lambda_{2}=0.6, \lambda_{3}=0.2$ at $b=0.4$. The main segments (faces of the parallelogram) are indicated by dashed lines. c) The return map $H$ for $s_{x_{1}=0} \rightarrow s_{x_{1}=0}$, rescaled to the unit interval, same parameters as in b).

one point at the face $s_{x_{1}=0}$ within the second or third iteration. Therefore by simple geometrical arguments one may conclude, that there are three ways leading to a mapping of the face $s_{x_{1}=0}$ onto itself. The dynamics of the system can be studied by a family of three segment piecewise linear continuous maps constituting the reduced return map $H: s_{x_{1}=0} \rightarrow s_{x_{1}=0}$. We denote the paths and give the slope $a$ of the associated branches of the reduced return map $H$ :

$g_{1}: s_{x_{1}=0} \stackrel{2}{\rightarrow} s_{x_{1}=b} \stackrel{1}{\rightarrow} s_{x_{1}=0}$

$\Rightarrow a_{1}=1$

$g_{2}: s_{x_{1}=0} \stackrel{2}{\rightarrow} s_{x_{2}=0} \stackrel{3}{\rightarrow} s_{x_{1}=b} \stackrel{1}{\rightarrow} s_{x_{1}=0}$

$\Rightarrow a_{2}=\lambda_{2} /\left(\lambda_{2}-1\right)$

$g_{3}: s_{x_{1}=0} \stackrel{2}{\rightarrow} s_{x_{2}=0} \stackrel{3}{\rightarrow} s_{x_{3}=0} \stackrel{1}{\rightarrow} s_{x_{1}=0}$

$\Rightarrow a_{3}=\lambda_{1} \lambda_{2} \lambda_{3} /\left[\left(\lambda_{1}-1\right)\left(\lambda_{2}-1\right)\left(\lambda_{3}-1\right)\right]$. 

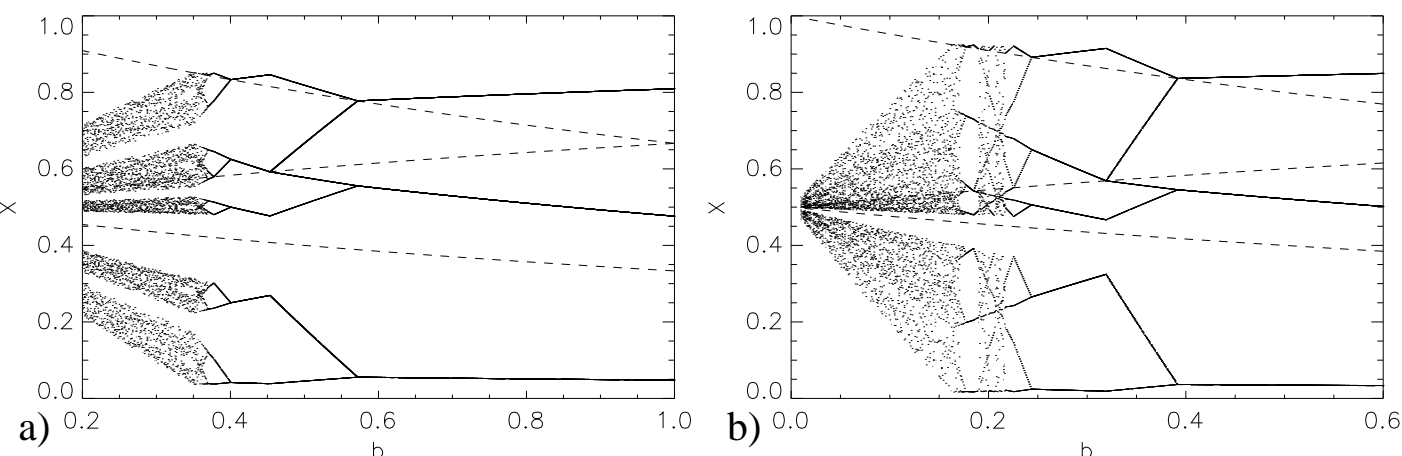

Figure 4.14: Bifurcation diagrams for an asymmetric three tank switched server system showing the normalized boundary coordinate $X$ vs. the upper threshold $b$ at the first tank. The dashed lines indicate the main segments $s_{x_{1}=0}, s_{x_{2}=0}, s_{x_{1}=b}, s_{x_{3}=0}$. The dynamics for the lowest segment in the bifurcation diagrams $s_{x_{1}=0}$ is given by the reduced return map. a) Filling rates $\lambda_{1}=0.2, \lambda_{2}=0.6, \lambda_{3}=0.2$, b) Filling rates $\lambda_{1}=0.1, \lambda_{2}=0.7, \lambda_{3}=0.2$.

The full equation of the reduced return map on the unit interval is:

$$
H\left(y_{n}\right)=y_{n+1}= \begin{cases}a_{1} y_{n}+d_{1} & \text { for } 0 \leq x(n)<c_{1} \\ a_{2} y_{n}+d_{2} & \text { for } \quad c_{1} \leq x(n)<c_{2} \\ a_{3} y_{n}+d_{3} & \text { for } \quad c_{2} \leq x(n)<1\end{cases}
$$

with: $a_{1}, a_{2}, a_{3}$ given above, $c_{1}=1-b\left(1+\lambda_{3} / \lambda_{1}\right), c_{2}=(1-b) / \lambda_{2}-b \lambda_{3} / \lambda_{1}$, $d_{1}=b \lambda_{3}\left[\left(1-\lambda_{1}\right)^{-1}+\lambda_{1}^{-1}\right], d_{2}=1+b \lambda_{3} /\left(1-\lambda_{1}\right)-c_{1} a_{2}-b$ and $d_{3}=\lambda_{3} /\left(1-\lambda_{1}\right)$. The branches $g_{1}$ and $g_{2}$ exist only if $c_{1}>0, c_{2}>0$, respectively. Thus existence and expansion of branches depends on $b$ again. The only expanding segment in the full Poincaré map is $g\left(s_{x_{2}=0} \mapsto s_{x_{1}=b}\right)$ provided that $\lambda_{2}>\lambda_{1}$. For the dynamics this expansion is effective in $a_{2}$ of segment $g_{2}$ in the reduced return map. For $\lambda_{2}<0.5$ a stable fixed point on $g_{3}$ or $g_{2}$ in the reduced return map is obtained, which corresponds to a stable period- 3 orbit in the full system. For $\lambda_{2}=0.5$ a stable period-3 orbit exists as long as $b \geq\left(1-\lambda_{1}\right) /\left(0.5+1.5 \lambda_{3}\right)$. For smaller $b$ a number of marginally stable orbits exist and for some parameter regions also stable higher periodic orbits occur (involving periodic points on $g_{3}$ in the reduced return map). If $\lambda_{2}>0.5$ the first bifurcation at $b=\left(1-\lambda_{1}\right) /\left(\lambda_{2}+\lambda_{3}+\lambda_{2} \lambda_{3}\right)$ is a period doubling border collision bifurcation. For smaller $b$ in this case further bifurcations and chaotic orbits occur. For small $b$, where segment $g_{3}$ is not admissible from $g_{1}$ or $g_{2}$ the dynamics is that of the skewed and shifted tent map consisting of $g_{1}$ and $g_{2}$. In this parameter region depending on $\lambda_{2} /\left(\lambda_{2}-1\right)$ chaotic interval attractors are obtained (see Sec.3.2.2).

Thus for the asymmetrically assigned threshold the possibility of the system to behave chaotically is determined by the value of $\lambda_{2}$. Figure 4.14 provides two examples of bifurcation diagrams for this system.

If the segment $g_{3}$ in the reduced return map is not reachable due to a small $b$ even a special dynamical situation is obtained. Whereas the hybrid system may be chaotic, 
the dynamics of the first, restricted tank remains periodic. It will be repetitively filled up to its maximum capacity and completely emptied afterwards. This behavior is shown in Fig.4.15.

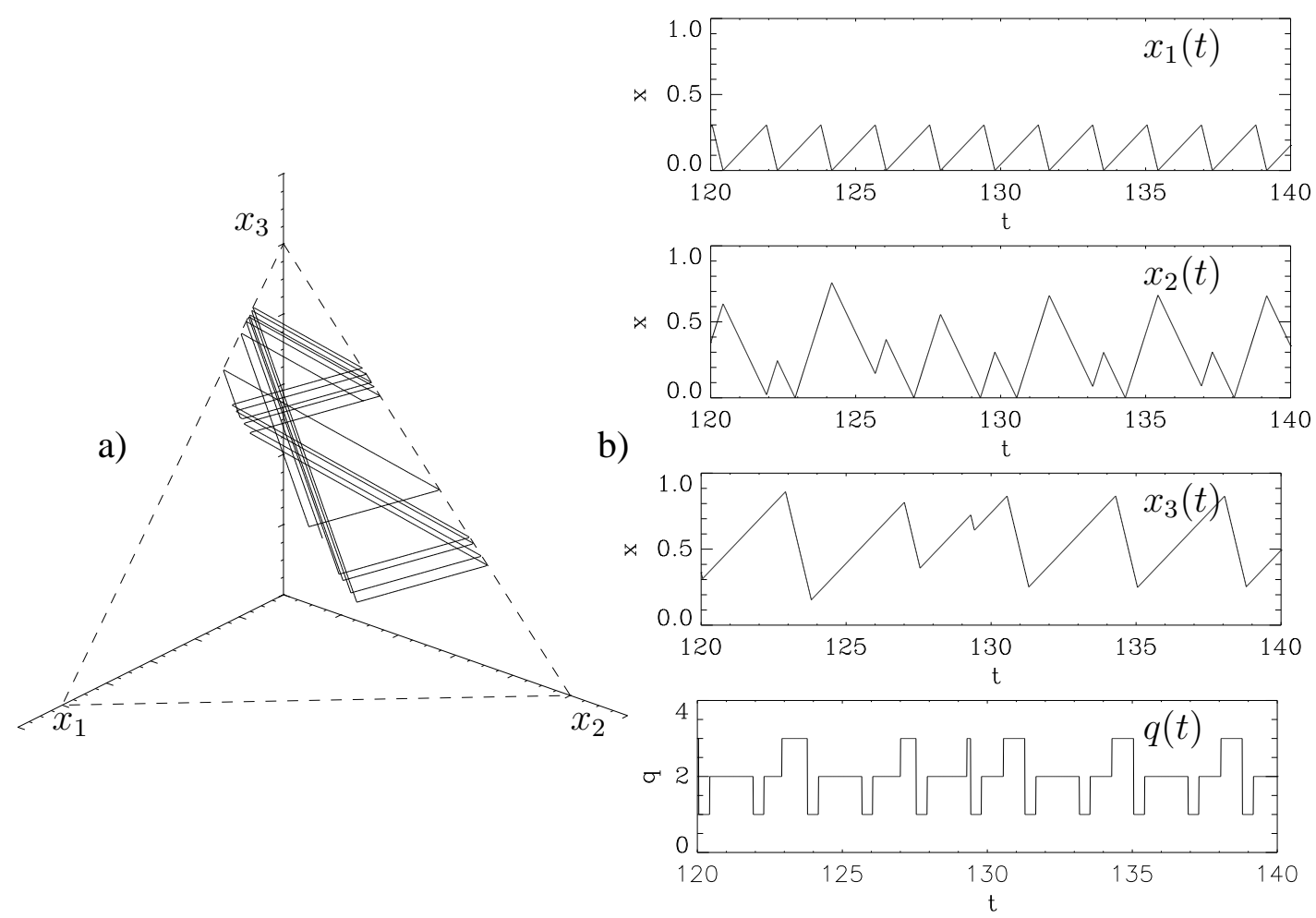

Figure 4.15: Example of the trajectory (a) and time series (b) of a three tank switched arrival system with an upper threshold $\left(b_{1}=0.3\right)$ at the first tank only. Parameters. $\lambda_{1}=0.2, \lambda_{2}=0.6, \lambda_{3}=0.2$. Note that the content of the first (restricted) tank fluctuates periodically whereas the whole system is chaotic (cf. Fig.4.14)

\subsubsection{A Three Tank System with Four Discrete States}

So far we have considered models of switched flow systems which involve one switching server. In the following we give an example of a system that involves two servers where each can be attached to only two tanks but both are connected through a common tank (Fig.4.16). Therewith we wish to emphasize two aspects. Firstly: Connections of switched flow systems can exhibit chaotic behavior even if subsystem are not able to show it (clearly, a two tank switched arrival system is not chaotic and a two tank switched server system also). Secondly: For more complicated systems the successive hits of the boundary may not be determined uniquely from a section of the boundary in the continuous part of the phase space but may depend on the foregoing discrete state $q$, too. So we have to use Poincaré maps with different leaves, or in other 
words: the Poincaré map becomes a hybrid map.

The switching rules are as follows:

Server I switches instantaneously, if one of the tanks A or B is empty to the empty one and begins to fill it up to the next switch.

Server II serves one tank, up to the moment where it is empty and starts then the service of the other one and so on.

We consider different filling $\left(\lambda_{i}\right)$ and emptying rates $\left(\mu_{i}\right)$ for each tank and set $\rho_{i}=$ $\lambda_{i} / \mu_{i}$. The system's dynamic is nontrivial if $\rho_{1}>1$ and $\rho_{3}<1$. Otherwise the server I can never leave tank $\mathrm{A}$, server II stays forever at tank $\mathrm{C}$ and the total content of the system approaches infinity.

We obtain four possible discrete states associated with four possible velocity vectors $\left\{v_{i} \mid i=1,2,3,4\right\}$ for the whole system, named $\mathrm{AB}, \mathrm{AC}, \mathrm{BC}$ and $\mathrm{BB}$, where the first capital names the tank that is filled by server I and the second the one, which is emptied by server II. Figure 4.16 b shows a state transition diagram for these four states with the parts of the boundary, where the transitions occur. The system is balanced if

$$
\rho_{2}=\frac{1-\rho_{3}}{1-\rho_{1}^{-1}}
$$

The fraction of time, where the arrival of server I is placed on A is determined by $\rho_{1}$, as well as the time, where server II serves $\mathrm{C}$ is determined by $\rho_{3}$. Because $\mathrm{B}$ can be filled by server I only during a fraction of time $1-\rho_{1}^{-1}$ and will be cleared in a fraction of size $1-\rho_{3}$ by server II the two flows $\rho_{1}$ and $\rho_{3}$ completely define the relation between $\lambda_{2}$ and $\mu_{2}$. As in the former models, the balance condition implies for this system, that the dynamics is restricted to a hyperplane in the continuous part of the phase space that is spanned by the velocity vectors $\left\{v_{i}\right\}$. With the switching conditions a normalized form of the bounded region can be given as $S^{E}=\left\{x \in \mathbb{R}^{3} \mid \sum_{i} c_{i} x_{i}=1, x_{i} \geq 0\right\}$ where $c_{i}$ are coefficients ${ }^{1}$ (depending on $\left.\left\{\lambda_{i}, \mu_{i} \mid i=1,2,3\right\}\right) \cdot \mathrm{S}^{E}$ is a generally not equilateral triangle.

Even for this system we obtain a Poincaré map by considering the mapping $\delta S^{E} \rightarrow$ $\delta S^{E}$ and a normalization to a map of the unit interval onto itself. Here the main segments of the map are given by the boundary segments of $S^{E}$ i.e. $s_{x_{1}}=0, s_{x_{2}}=0$, $s_{x_{2}}=0$, wher the switching processes of server I and II take place. An example of this map is depicted in Fig.4.17. The Poincaré map for this system is not unique. It is hybrid, because the branch of the map that must be used for an iteration depends on whether the upper path of the state transition diagram (Fig.4.16b) is recently used or

\footnotetext{
${ }^{1}$ We obtain $c_{1}=n\left[\lambda_{1} \lambda_{2}\left(\lambda_{3}-\mu_{3}\right) /\left(\mu_{1}-\lambda_{1}\right)\right], c_{2}=n\left[\lambda_{1}^{2}\left(\lambda_{3}-\mu_{3}\right) /\left(\mu_{1}-\lambda_{1}\right)\right], c_{3}=n\left[\lambda_{1} \lambda_{2}\right]$, where $n$ is a normalization factor such that $1=\left[c_{1}^{2}+c_{2}^{2}+c_{3}^{3}\right]^{1 / 2}$.
} 
a)

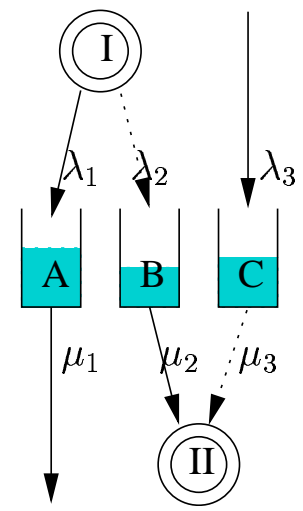

b)

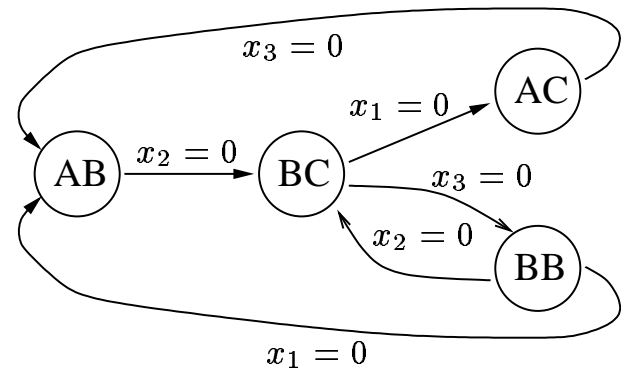

Figure 4.16: Scheme (a) and graph representation of the symbolic transitions (b) for a switched tank system involving two switching servers

the lower one. We denote these paths by the discrete state transitions that are made, i.e $(B C, A C, A B)$ for the upper one. The decision between the paths depends on the subsegment of $s_{x_{2}=0}$ to which the system is mapped in state $A B$. We can simplify the complete Poincaré map by introducing a reduced branch ${ }^{2} H: s_{x_{2}=0} \stackrel{(B C, A C, A B)}{\longrightarrow}$ $s_{x_{2}=0}$, shown by a dashed green line and labeled with $(B C, A C, A B)$ in Fig.4.17, summarizing the mapping under the upper path of discrete state transitions. This reduced branch is crucial for the dynamical behaviour, therefore we give its slope:

$$
a_{(B C, A C, A B)}=\rho_{1} \rho_{3} .
$$

The reduced branch intersects the diagonal $(X(n)=X(n+1))$ if

$$
\rho_{1}^{-1}+\rho_{3}>1 \text {. }
$$

It turns out, that the dynamics of the system depends crucially on the choosen parameters $\left\{\lambda_{i}, \mu_{i}\right\}$. We shall discuss some possibilities.

Case 1: $\rho_{2}>1, \rho 1^{-1}+\rho_{3}>1$

If $\rho_{2}>1$ the content of tank $\mathrm{B}$ grows as long as the system is in state $\mathrm{BB}$. The edge $B B \stackrel{x_{2}=0}{\longrightarrow} B C$ in the state transition diagram (Fig.4.16b) is never reached.

If condition 4.14 is fullfilled, then there exists a stable fixed point for the mapping $H: s_{x_{2}=0} \stackrel{(B C, A C, A B)}{\longrightarrow} s_{x_{2}=0}$, because from Eq.(4.13) and Eq.4.12 with $\rho_{2}>1$ follows that $\left|a_{(B C, A C, A B)}\right|<1$. Thus we obtain a stable periodic orbit of the system in this case. Fig.4.18 shows an example. For the system the state BB is under these conditions a "garden eden" state, a state that can only occur if given as initial condition and

\footnotetext{
${ }^{2}$ With the introduction of this reduced branch the hybrid Poincaré map becomes a unique mapping of the unit interval onto itself.
} 


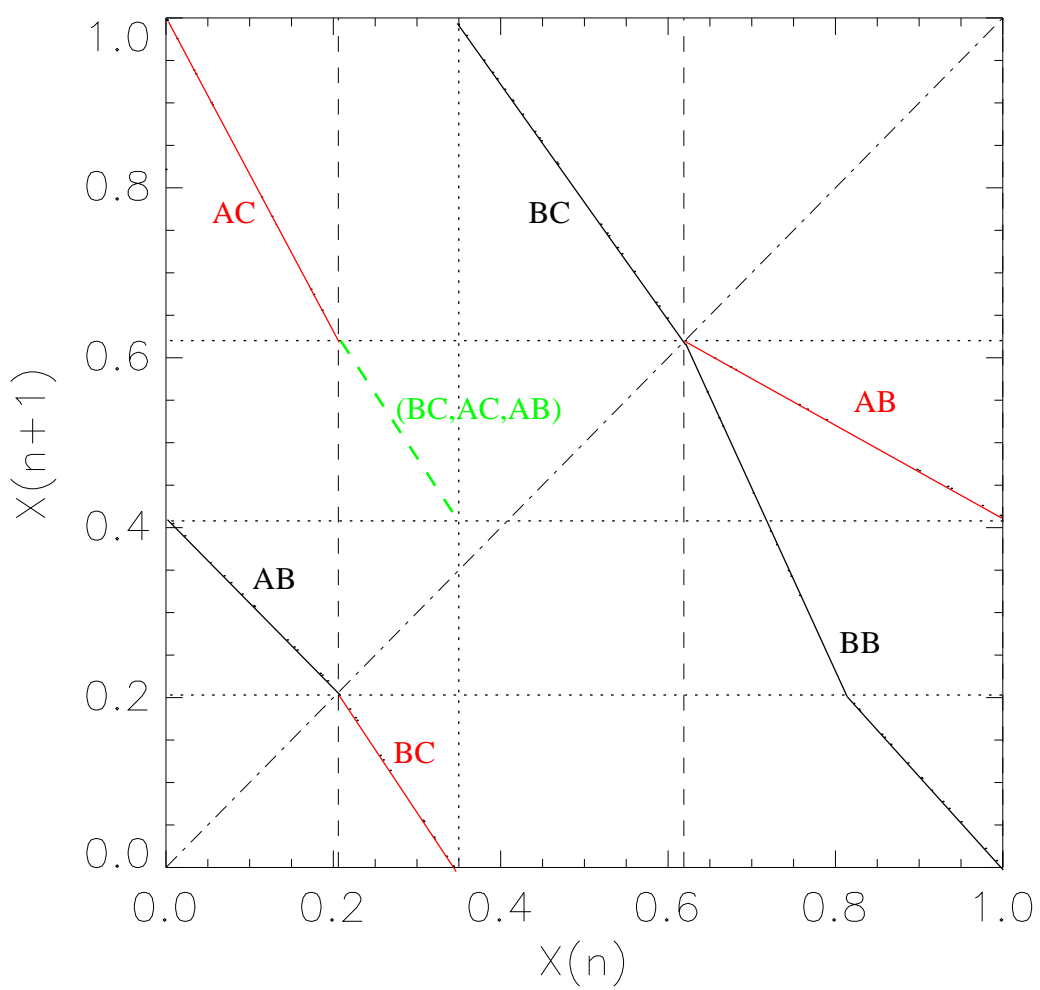

Figure 4.17: Poincaré map for a switched tank system involving two switching servers with $\lambda_{1}=3.0$, $\lambda_{2}=0.75, \lambda_{3}=1.0, \mu_{1}=1.0, \mu_{2}=1.0, \mu_{3}=2.0$. The main segments of the boundary $\left(s_{x_{1}=0}\right.$, $\left.\mathbf{s}_{x_{2}=0}, \mathbf{s}_{x_{3}=0},\right)$ are indicated by dashed lines. The Branches are labeled with the symbolic states for which the transitions occur. The red branches correspond to an symbolic orbit $(\mathrm{BC}, \mathrm{AC}, \mathrm{AB})$ in the transition graph (the upper path in Fig.4.16b). The dashed green branch is the summarized result of this path. For a iteration of this hybrid Poincaré map the transition graph Fig.4.16b must be considered to choose the next branch, where two possibilities are given.

then never reached again.

Case 2: $\rho_{2}=1$

Even if $\rho_{2}=1$ the edge $B B \stackrel{x_{2}=0}{\longrightarrow} B C$ can not occur, moreover in state $\mathrm{BB}$ the content of tank B remains constant as in the state AC. In other words $v_{B B}=-v_{A C}$ and both velocity vectors are parallel to the edge $s_{x_{2}=0}$ of the triangle in the continuous part of the state space. The assumption of equal inflow and outflow for the second tank $\left(\rho_{2}=1\right)$ implies that the relation between in- and outflow for the first tank A meets the relation of out- and inflow for the third tank $\mathrm{C}\left(\rho_{1}=\rho_{3}^{-1}\right)$.

In this case periodic orbits with a symbolic sequence composed of $(B C, A C, A B)$ and $(B C, B B, A B)$ sequences are obtained. A special situation occurs if $\rho_{1}^{-1}=\rho_{3}=1 / 2$. This implies that the velocity vectors $v_{A B}$ and $v_{B C}$ are parallel and of opposite direction in the strange billiard. Thus every orbit $(A B, B C, B B, A B, B C, A C)$ runs the 

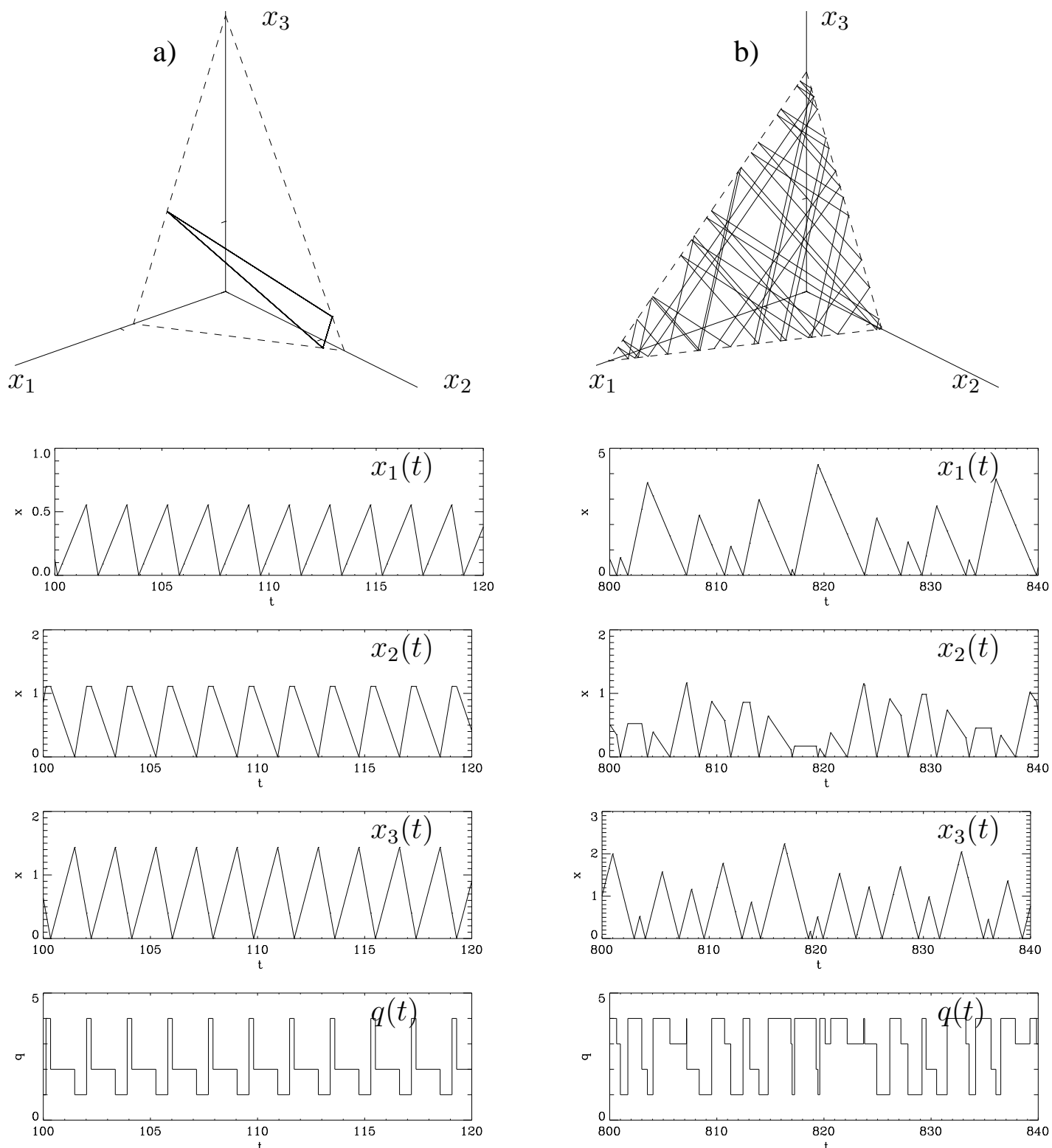

Figure 4.18: Sample of trajectories and time series for the switched tank system with two switching servers, illustrating the $\rho_{2}>1$ case (first column) and the $\rho_{2}<1$ case (second column).(a): $\lambda_{1}=2^{1 / 2}$, $\lambda_{2}=2.0, \lambda_{3}=1.2750 \ldots, \mu_{1}=1.0, \mu_{2}=0.9859 \ldots, \mu_{3}=\pi .,(b): \lambda_{1}=3.0, \lambda_{2}=0.75, \lambda_{3}=1.0$, $\mu_{1}=1.0, \mu_{2}=1.0, \mu_{3}=2.0$.

same way back and forth in the continuous part of the phase space, and all orbits of this form are marginally stable.

Case 3: $\rho_{2}<1$. 
Only if the second tank can become empty even in state BB the full transition diagram of Fig.4.16b applies to the system. In this case the system is chaotic. See Fig.4.18 for an example.

\subsection{Summary}

We have analyzed the dynamics of two basic types of hybrid tank systems with switching servers from the viewpoint and with methods of Nonlinear Dynamics. Due to the continuity of the trajectory at the switching points in the continuous part of the state space their behavior can be characterized as a strange billiard. In Sec.4.2 we have shown that with the introduction of upper thresholds switched arrival and switched server systems, two previously as contrasting examples for the dynamics of switched flow systems described types are limit cases in one and the same system and reciprocal. This seemingly simple relation was not recognized before. For systems with three tanks these limit cases show chaotic and periodic dynamics both in switched server and switched arrival systems.

By sampling the dynamics at the switching points for systems with three tanks one dimensional piecewise linear Poincaré maps were obtained and discussed in detail. When the bifurcation parameter $b$ is varied, linear segments of the Poincaré map appear, disappear and change their extension whereas the slope of the segments is constant. We have demonstrated that the change of extensions and the associated shift of segments leads to bifurcations in the strange billiard. These bifurcation scenarios are built (exclusively) from sequences of several border collision bifurcations. Sometimes these bifurcations arise in a rapid sequence at different segments of the Poincaré map. These scenarios are therefore very different from the usual bifurcation scenarios, obtained for smooth maps on the unit interval. The dynamics here is governed by an interplay of expanding and contracting properties in connection with discontinuities of the Poincaré maps. Typical bifurcation mechanisms are discussed and illustrated with bifurcation diagrams. For a detailed study of bifurcations in certain intervals we used reduced return maps which are often simpler than the full Poincare map. This technique may be useful in other cases too.

For more complicated systems such as those considered in Sec.4.4.3 the successive hits may not be determined uniquely from a section of the boundary in the continuous part of the state space anymore but depend on the foregoing discrete state $q$ too. In this case we obtain hybrid Poincaré maps.

Here we did not consider any higher dimensional system with more than three tanks. Such systems will lead in a natural way to higher dimensional piecewise linear maps with even more complex dynamics. 



\title{
5 Modeling of Manufacturing Systems
}

\begin{abstract}
In this chapter modeling of manufacturing systems as an application of hybrid models is discussed. Specifically we investigate the previously introduced switched server and switched arrival systems for the interdependencies between switching policies, switching thresholds and the logistic performance.

After a general introduction concerning some aspects of modeling manufacturing systems at a logistic level as dynamical systems (Sec.5.1) we introduce some extensions to the switched server and arrival systems to include set up times which are often unavoidable in manufacturing (Sec.5.2). In Sec.5.3 an analysis of the performance of this dynamical systems from a logistical point of view is done. Finally, in Sec.5.4 we present numerical evidence, that the dynamics of discrete deterministic queuing models is similar to the dynamics of hybrid models.

In the context of manufacturing systems our investigations lead not only to the conclusion that for a given layout and policy the dynamics of a system may be affected significantly by restricted buffer sizes, but also shows the complex relation between system parameters, dynamics and performance.
\end{abstract}

\subsection{Manufacturing Systems and Hybrid Models}

The basic unit of most manufacturing systems is a work station containing a server (e.g., a machine) to perform some processing and a buffer, queuing the material before it can be processed. In practice the meaning of servers and material may be very different from system to system. A server may represent a machine or a group of machines (dubbed "job shop" some times) in which case the material are work pieces processed in a factory. Otherwise the "material" may be a group of passengers for a scheduled flight, waiting for an aircraft, or at another level, some aircrafts that have absolved a number of flights, and are waiting for a maintenance phase. Or the material are data packets originating from a computer network, that have to to be scheduled to their destination.

From these randomly picked examples it is obvious that a large number of man made systems, which can be considered even at different levels of coarse-graining (one may think of a hierarchy like machines, job shops, factories, supply chains) fit into the gen- 
eral description.

However, modern manufacturing systems are typically large networks of various production and storage facilities. Their dynamics is governed by complex, system immanent interdependencies involving both deterministic and stochastic elements as well as unforeseen external influences. A basic feature of most manufacturing systems is the existence of policies, controlling the allocation of scarce resources among competing tasks. It is of great importance for operators of such manufacturing systems to know how the policies may influence the time evolution or even the performance and profitability of the manufacturing process. Also knowledge of the dynamics of such processes is urgently needed for planning and control of manufacturing.

Traditionally the investigation of manufacturing systems is addressed in operations research, mainly in the context of a large area called queuing theory. The classical queuing theory focuses on the influence of stochastic arrival and departure processes on the formation of queues, mostly in the language of equilibrium solutions. However, in the framework of queuing theory the evolution of systems in time is hardly to obtain and the distributions of inter-event times in modern manufacturing, which are often restricted and have small variances are difficult to handle in a stochastic formulation. Thus a number of recent investigations came up with the description of manufacturing systems as deterministic dynamical systems to overcome the restrictions of queueing theory and develop a new framework for efficient modeling and analysis of industrial processes (see [22] and references therein).

In the context of dynamical systems rule (policy) dependent switchings between different operation modes (and parameters) are essential nonlinearities, leading to complex behavior in manufacturing systems. To analyze such influences it is reasonable to neglect any stochastic influence and the discreteness of the material flow. This approach leads to dynamical systems consisting of piecewise defined continuous time evolution processes interfaced with some logical or decision making process. These approximations lead in a natural way to hybrid dynamical systems as models of manufacturing systems.

With a hybrid modeling approach we shall not try to account the "complexity" and confusing structure of interactions that arises in a 1:1 model of a large production facility. In particular for investigations of mainly stochastically determined behavior that characterizes production systems which are strongly influenced by humans and sudden random breakdowns of installations queuing theoretical methods are naturally the best choice. But individual automated subsystems are comparatively well defined and seem to be promising candidates for a hybrid modeling approach. 

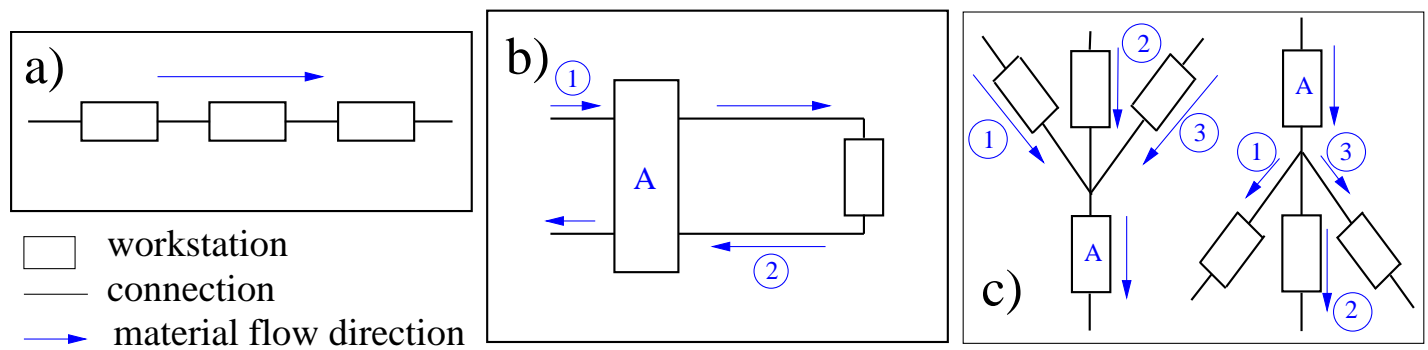

Figure 5.1: Diagrams of basic structures of automated production lines, after [66]. a) Linear, b) Reentrant systems: Server $A$ needs a policy to decide whether stream 1 or stream 2 should be processed. c) m:n junctions, here referred to as switched server and switched arrival systems, station A needs a policy to decide which of the following stations should be filled or which of the foregoing stations should be served.

Such automated production lines are networks, build from very few basic concatenation principles [66]. Fig. 5.1 shows diagrams of tree basic types. Besides pure sequential configurations and reentrant structures also so called $m: n$ junctions where one machine has to serve a number of foregoing workstations or one workstation has to deliver products to some subsequent stations appear. Most of the real world m:n junctions are $2: 1,1: 2$ or $1: 3$ and $3: 1$ catenations [106]. The latter configuration shall be investigated here. Inside a larger production facility these basic configurations may occur in several combinations in the same production network. However, any scientific approach should at first try to understand the dynamical features of such basic configurations.

\subsubsection{A Brief History of Hybrid Modeling for Manufacturing Systems}

One basic idea of hybrid modeling, the approximation of the discrete flow of items by a continuous flow which can be described by a differential equation is not new. It was frequently used as simple example in queuing theory (see [64], vol.II ) as well as in the production engineer's community where it is also known as "funnel approach" [82].

To our knowledge, the first work that used a hybrid modeling approach to study the dynamics of a system that falls in the above outlined class of manufacturing systems appeared in 1991. In [45] a hybrid model for telephone traffic switching systems incorporating two buffers, a switching server and a time delayed feedback loop was analyzed by means of Poincaré map techniques and oscillatory behavior and chaos were found. The paper closes with the programatic words: "More generally, there is a need to view teletraffic systems as dynamical systems whose behavior can substantially influence performance."

The investigation of deterministic models of manufacturing systems received a further 
impulse from the pioneering works of Beaumariage and Kempf [13] who observed in a number of simulations of simple models of reentrant structures a sensitivity to initial conditions and parameter changes that led to the conjecture of chaotic behavior in these systems. In a number of following investigations [41, 42, 55] this conjecture was proofed to be invalid, but it was demonstrated that methods of nonlinear dynamics can be successfully applied to understand the dynamics of manufacturing systems.

$\mathrm{M}$ to $\mathrm{n}$ connections in manufacturing systems in form of switched server and switched arrival systems where investigated in Chase et al. [25] and in [92, 98, 59, 63, 62, 87] (see Chap.4). Whereas all these works were motivated partly by the occurrence of switched server structures in manufacturing systems the consequences of dynamics for the performance were addressed only in $[63,62,87]$ for the switched arrival system with unrestricted buffers.

Another field of manufacturing systems for which hybrid models were successfully considered is the understanding of supply chain dynamics. The problem making the management of supply chains a challenging task is the delay between an incoming order (orientated at the actual demand) and the delivery date. This delay causes usually a mismatch between the delivered amount of goods and the actual demand (which is less or higher than the previous one). Supply chains can be modeled by hybrid systems with timed events, since the orders are made usually at fixed time instants and the delivery will also be done at fixed times. The processing of orders, however, is a continuous process. A basic model also known as the "beer game" was studied by means of a hybrid modeling approach (see [68] and the references therein).

Last but not least the works $[12,11,22]$ on deterministic hybrid models of different forms of the Toyota Sewn Products Management System (TSS) should be mentioned here. In principle the question is addressed how the work of a limited number of workers at a street of (sewing) machines should be organized, if the worker carry their workpieces from machine to machine, usually with different work speeds, since they are more or less skilled. For such systems where the workers are sequenced from slowest to fastest, the term bucket brigade was coined by Bartholdy and Eisenstein [12]. In [11] by hybrid modeling piecewise smooth maps for two and three worker systems ${ }^{1}$ where derived and for a two worker system a fixed point could be identified that corresponds to an optimal production rate.

\subsubsection{Switched Arrival and Server Systems in Manufacturing}

As mentioned above (see Fig.5.1) layout structures where one work unit has to load a number of subsequent units or one unit has to server some foregoing workstations appear as basic concatenation principles in manufacturing. Because the parallel (foregoing or subsequent) work units in general may process different products such connections are generally operated under scheduling policies summarized under the term

${ }^{1}$ Many real life systems operate with only a few workers. 


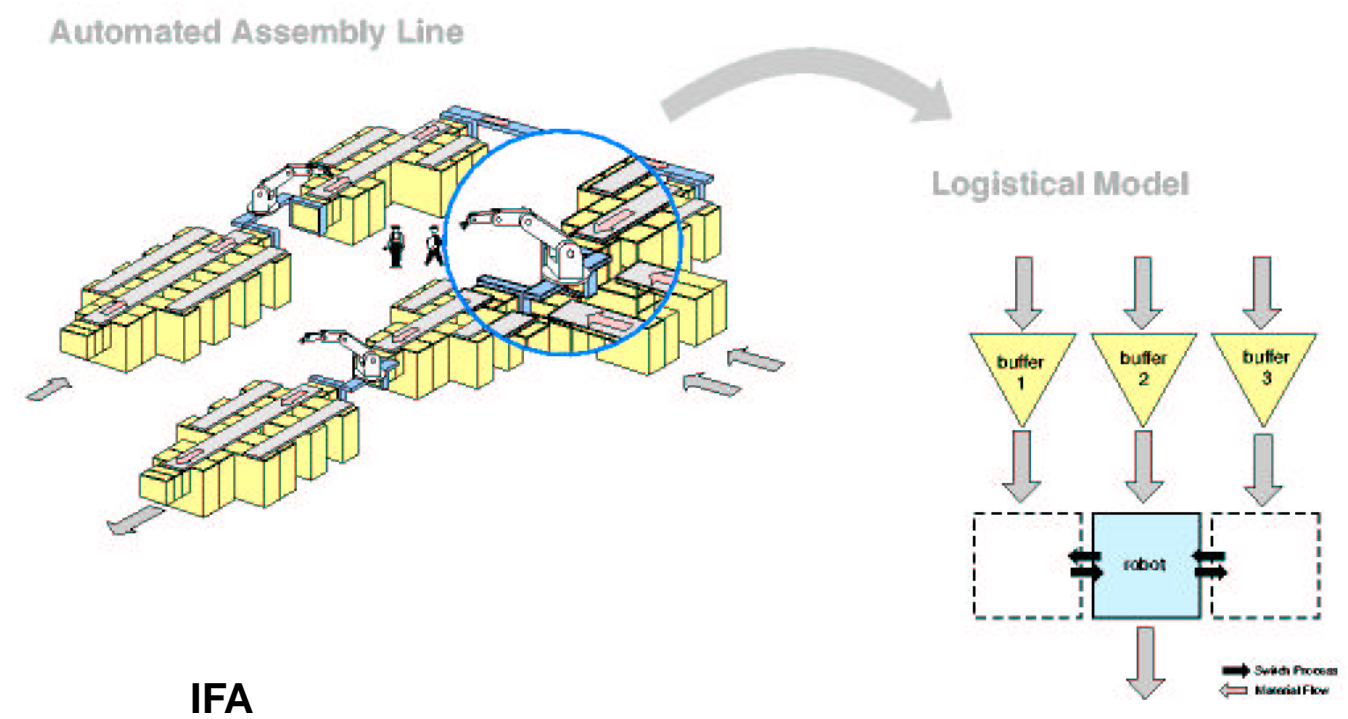

Figure 5.2: Part of a production line for crankshafts as example for a switched arrival system [107].

CAF "clear-a-fraction". That means the server picks one of the parallel buffers and serves it at its full ability until a switch to another buffer is required by any rule. In Chap. 4 we have investigated such configurations as switched arrival systems and switched server systems. In particular the balanced regime was considered, with a total inflow into the systems that meets the total outflow. The balanced state guarantees the normal operation of a manufacturing system and is in industrial engineering assured under CONWIP (Constant work in process) policies. The upper limits applied to the tanks in Chap. 4 are translated here in buffers with a restricted maximum capacity. And buffers in manufacturing are allways of limited capacity.

We shall now review the main results from the investigations in Chap.4 in this context. For switched arrival configurations that are chaotic for large buffer capacities, smaller buffer sizes lead to a more regular (periodic) behavior. In switched server configurations with three (or more) buffers small buffer capacities lead to chaotic behavior. The onset of chaos can be very abrupt (cf. Fig.4.5f).

These results coincide with operational experience aggregated by production engineers [106]. Despite of the fact that basically small buffers are favorite to keep the inventory small and to reduce throughput times, it is empirically well known that in some configurations to small dimensioned buffers cause several problems.

For a given configuration the behavior can be changed qualitatively (from chaotic to regular dynamics for instance) by slight changes of policies (Sec.4.4.1). In view of the parameter dependence of dynamics it depends on the given configuration whether those measures are succesful and which rules should be choosen. 
The switched server system with only one upper threshold Sec.4.4.2 is a model for a manufacturing system with three (or more) priority classes.The first (restricted) buffer is has the highest priority in this model.

\subsection{Manufacturing Systems with Set-up Times}

Usually different buffers (i.e. tanks in hybrid models) contain different types of products. Thus in general a server incurs a "set-up time" $\tau$ (a bounded delay) when changing over to serve a new tank. A set-up is needed to change tools, to make the switching itself or to perform maintenance which would be scheduled if a buffer is not served. During this time interval $\tau$ the server is not able to work at its full ability causing production losses and finally additional costs which are discussed in the following section.

\subsubsection{A Model for Switched Server Systems with Set-up Time}

One way to take into account losses caused by set-up times is to assume a fixed amount of costs for each switching. This implies in some sense that the whole system is stopped for a time $\tau$ and continues after this interval just at the same state it had before the switching took place.

On the other hand it is desirable to improve the switched server (arrival) models by introducing an effective reduction of output in connection with switching events. Therefore one may assume that a server can work during a certain setup time, but with a reduced rate. To conserve the balanced state of the system a simultaneous reduction of the total in- and outflow of the system is necessary.

Recently an additional switching rule ${ }^{2}$ was proposed for the switched arrival system without restricted buffer capacities [87] for this purpose. If restricted buffers or switched server systems are considered, this proposed rule is not feasible.

We consider here switched server systems with three buffers and the following

${ }^{2}$ This rule can be formulated as:

switched arrival systems, no upper threshold:

If a buffer $(i)$ runs empty let the server for a certain time $\tau$ stay filling the "old" buffer, but with a reduced rate $\lambda^{*}=1-\mu_{i}$ which matches the total outflow of the remaining (non-empty) buffers. After $\tau$ switch the filling server to buffer $i$. The filling rate recovers its normal value $\lambda=1$. 
a)
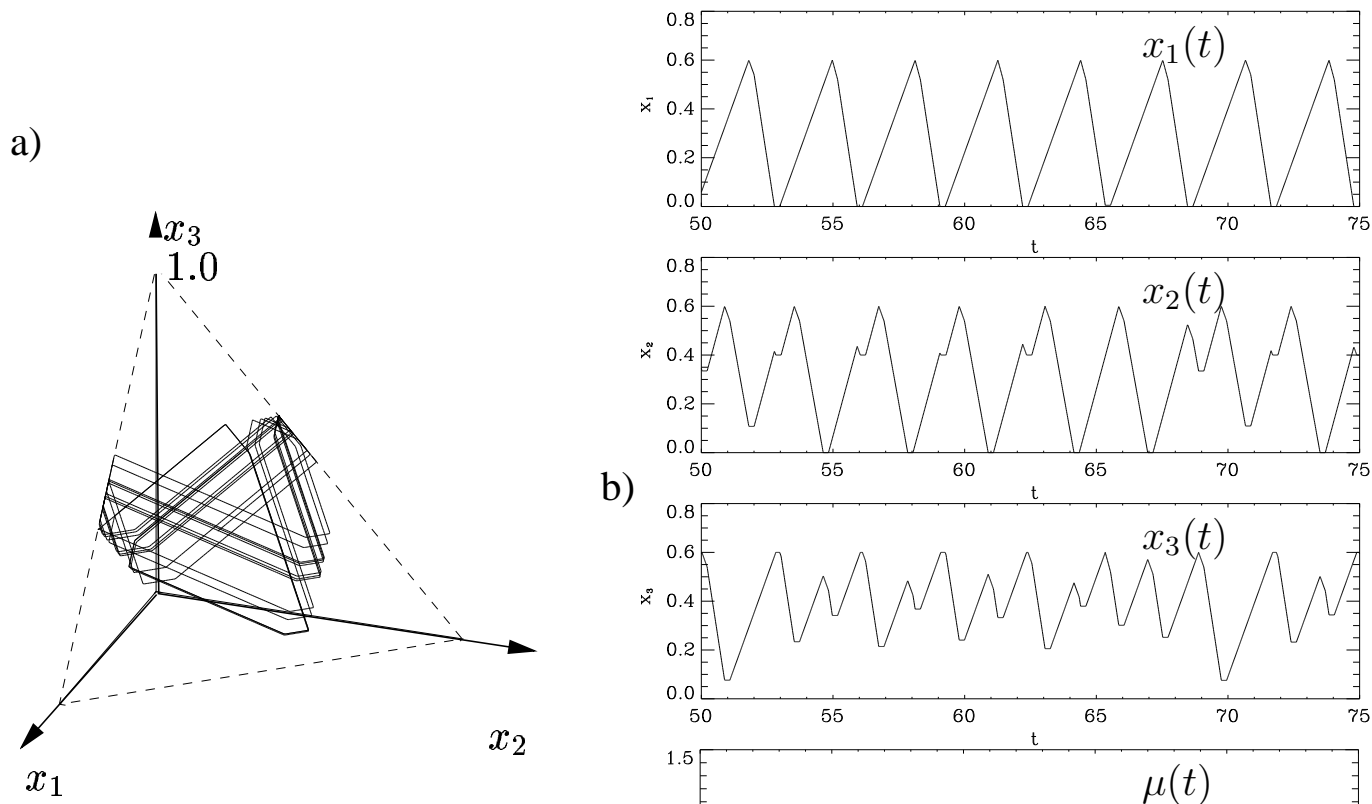

b)
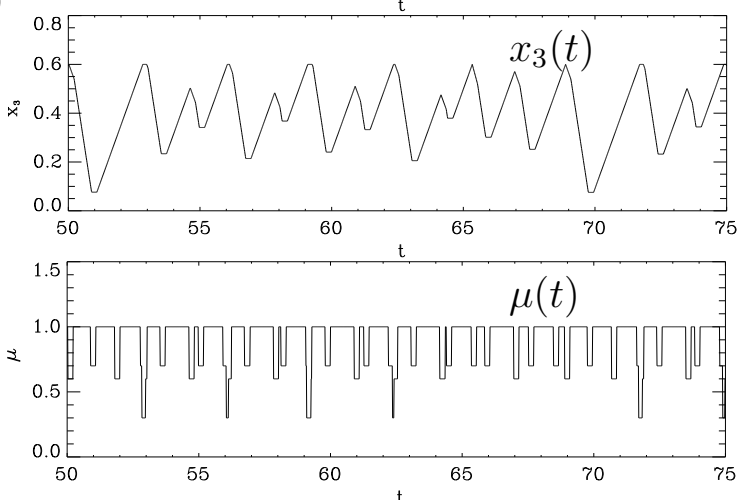

Figure 5.3: Dynamics of a three tank switched server model $\left(\lambda_{1}=0.3, \lambda_{2}=0.4, \lambda_{3}=0.3\right)$ with an additional set-up time $\tau=0.2$. The rule 5.1 is applied. a) Sample trajectory of the tank content at $b=$ 0.6 . b) Short time series of the buffer contents $x_{i}(t)$ and the production rate $\left(\lambda(t)=\sum \lambda_{i}(t)=\mu(t)\right)$ for the system if the maximum tank content is $b=0.6$. Every time a switching is made, the production shrinks.

additional switching rule:

If required, switch the server according to the rules given in Sec.4.

During a time $\tau$ after a switching maintain the just left tank at its previous level, i.e. this tank gets no input for the period of time $\tau$. Also the emptying rate $\mu$ of the server is reduced by the rate that normally applies to this just left tank.

This rule is applicable even for switched arrival systems and more than three buffers. It may be also combined with different switching rules for the lower threshold in switched server systems (Sec.4.4.1). For simplicity we require an identical $\tau$ for all switches. With the introduction of a set-up time timed events (see Sec.2.1.1) are introduced in the hybrid system, which previously was governed by state events only.

Furthermore, the additional rule implies a number of new discrete states for the hybrid system. Besides the states $q=\{i \mid i=1,2,3\}$ for three buffers where the server is attached to buffer $i$ and empties it with rate $\mu_{i}=1$ in the normalized system, 


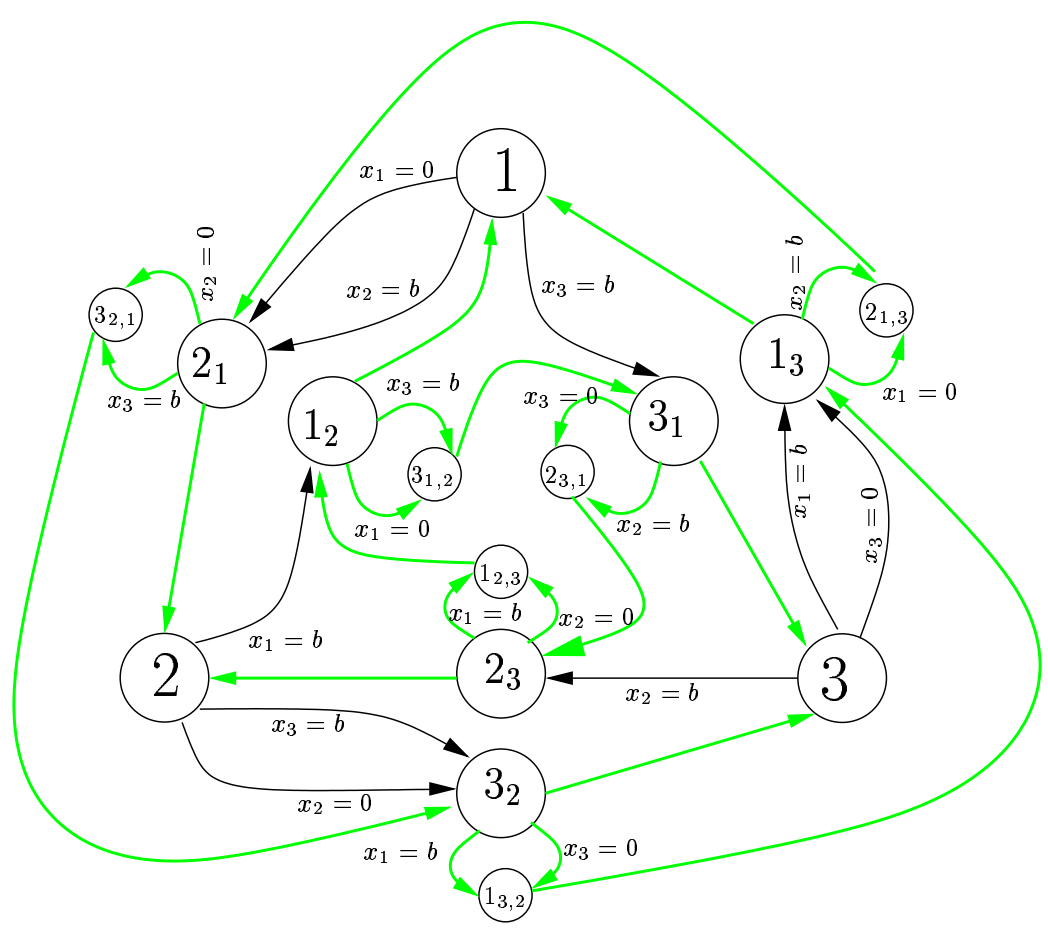

Figure 5.4: State transition diagram of a three tank switched server model with a maximum buffer capacity $b$ and an additional set-up time. The discrete states are labeled as described in the text. Transition edges that are associated with state events are inscribed with this state event. The green edges (without in-scripts) correspond to time event transitions. Note that in the discrete states $i_{j, k}$ (small circles) the continuous state of the system remains constant.

six further discrete states occur where one tank is not filled (during the set-up time following a switching). These states are denoted by $q=\left\{i_{k} \mid i, k=1,2,3: i \neq k\right\}$ where the number $(i)$ gives the buffer to which the server is attached and the subscript $(k)$ denotes the (just left) buffer that is out of filling. In these states the emptying rate, i.e. the output rate of the system is reduced to $\mu_{i_{k}}=1-\lambda_{k}$ and in the continuous part of the phase space the associated velocity vectors are parallel to side $s_{x_{k}=0}$, because the content of buffer $k$ stays constant for the period $\tau$. At the end of the time interval $\tau$ after a switch the discrete state jumps back $\left(i_{k} \mapsto i\right)$, but it may happen that previously the continuous state hits another boundary segment of $S_{3}^{*}(b)$. In this case the server switches, but for the remaining part of the interval $\tau$ now whether the previously left tank nor the recently left tank obtain input and the emptying rate associated with this new discrete state, denoted by $j_{i, k}$, is $\mu_{j_{i, k}}=1-\lambda_{i}-\lambda_{k}=\lambda_{j}$. In other words, only one tank is filled and emptied with the same rate and the continuous state of the system is constant. Thus every switching process in the system is followed by a period of reduced production rates. Figure 5.4 shows the graph of state transitions for the three tank switched server system with set-up times applied according to rule 5.1. 

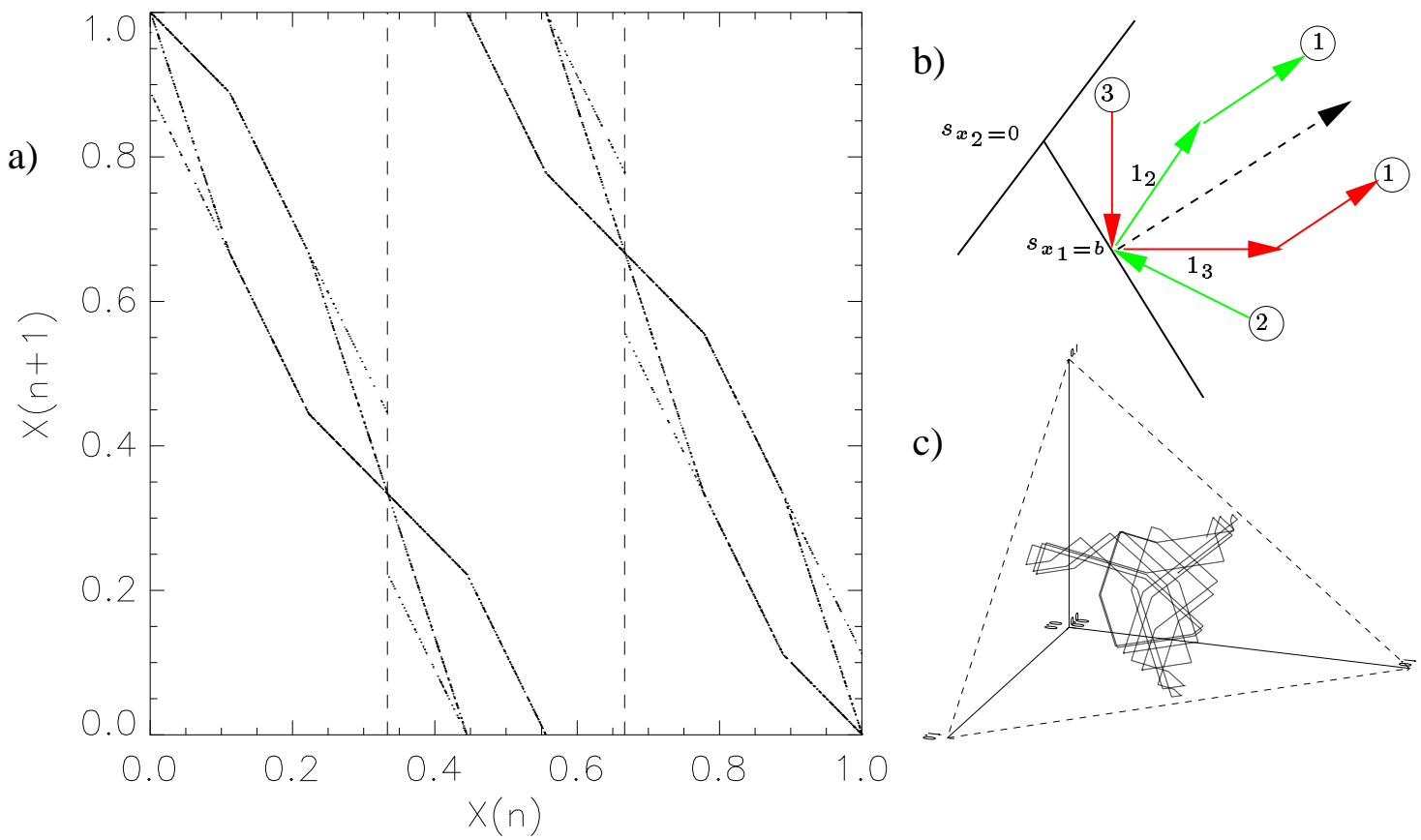

Figure 5.5: Poincaré map (a) of a three tank switched server model $\left(\lambda_{1}=\lambda_{2}=\lambda_{3}=1 / 3\right)$ with an additional set-up time $\tau=0.5$ and a maximum buffer capacity $b=0.5$. The rule 5.1 and a cyclic switching 4.2 at the lower threshold is applied. The main segments (corresponding to faces of $\tilde{S}_{3}$ ) are indicated by dashed lines.b) Different velocity vectors during the set-up time lead to an up and downward shift of segments in the Poincare map, depending on the foregoing discrete state. Without a set-up time both depicted cases will be uniquely mapped under the dashed vector. c) Corresponding sample of the trajectory in the continuous part of the state space.

\subsubsection{Poincaré Maps and Dynamics}

The additional rule implies a number of new discrete states for the hybrid system (Fig.5.4). For three tanks we obtain six additional states where one tank is out of service and six further states ${ }^{3}$ where two tanks simultaneously are out of service. Therefore the introduction of a set-up time with reduced production rate also changes the dynamical behavior (cf. Fig.5.6). Furthermore, the switches between different discrete states occur not only at the boundary $\delta S_{3}^{*}$ and the following discrete state depends on the previous one.

Since the following hit of the boundary $\delta S_{3}^{*}$ in the continuous part of the state space is uniquely determined by the previous discrete state and the boundary coordinate a

\footnotetext{
${ }^{3}$ Since there are only three different emptying rates associated with these six states and the velocity vectors in the continuous part of the state space are zero in all six cases, one may conclude that there are only three or one states, respectively. However, to make the graph representation unique, we need the full number of six discrete states with two buffers out of service.
} 

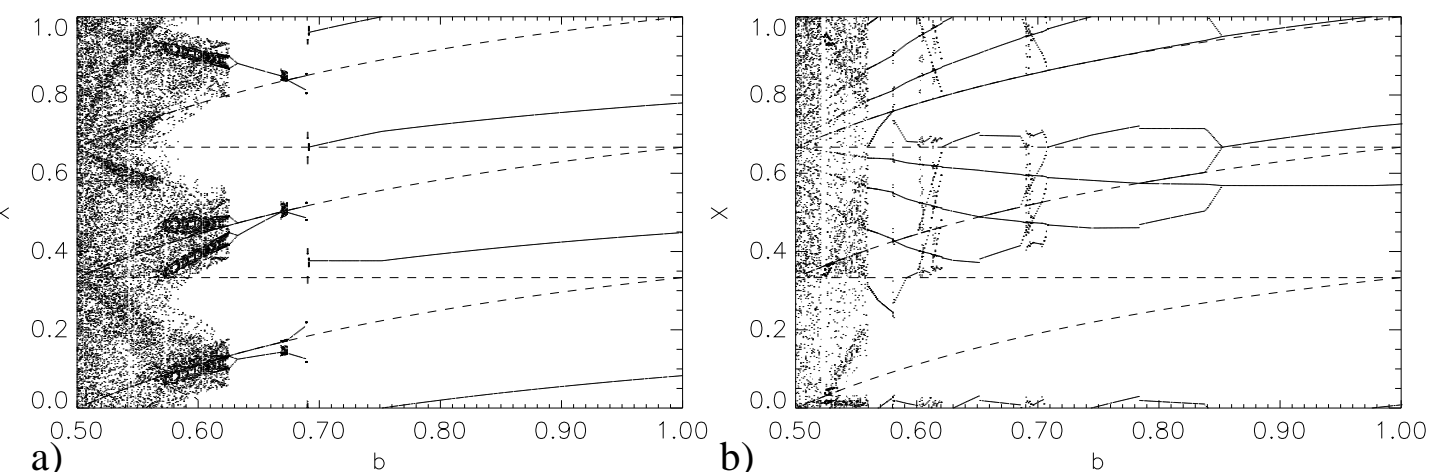

Figure 5.6: Bifurcation diagrams of three tank switched server models with an additional set-up time. The rule 5.1 and a cyclic switching (rule 4.2) at the lower threshold is applied. The main segments (corresponding to faces of $\tilde{S}_{3}$ ) are indicated by dashed lines. a) $\lambda_{1}=0.0, \lambda_{2}=0.4, \lambda_{3}=0.3$, set-up time: $\tau=0.2$. b) $\lambda_{1}=0.15, \lambda_{2}=0.8, \lambda_{3}=0.15$, set-up time: $\tau=0.5$.

-now hybrid- Poincaré map for the mapping $\left(\delta S_{3}^{*}, Q\right) \rightarrow\left(\delta S_{3}^{*}, Q\right)$ can be obtained. In the hybrid Poincaré map for the same normalized boundary coordinate $X \in(0,1)$ two and three separate branches appear, depending on the number of discrete states that can lead to a mapping to this value of $X$. Figure 5.5a gives one example of such a Poincaré map. In middle positions of the faces, that can be reached only by states with all buffers filled continuously, only two branches are present, one for each of the possible discrete states. The slopes of these branches are the same as for the switched server model without set-up times, but they are shifted upwards and downwards now (see Fig.5.5b for an explanatory sketch). Only near vertices the continuous state of the hybrid system may reach a new face of the boundary during the set-up time. In this case also the third possible discrete state appears as a preceding state and a third branch in the hybrid Poincaré map is visible.

The consequences of set-up times for the low periodic orbits (which are typical for large $b$ in the switched server system) are not important because the orbits are only slightly shifted compared to the systems without set-up times. But for smaller $b$ the dynamics is modified. Nevertheless for $b$ approaching the limiting case $b=0.5$ systems with set-up times are chaotic. One may state, that the dynamics of switched server systems with set-up times is similar to the systems without this set-up. Fig.5.6 gives examples of bifurcation diagrams for these systems.

We remark that in the model with set-up times the system can also stay at vertices of $S_{3}^{*}$ during set-up times with states $i_{j, k}$ and then evolve forward during the following $i_{j}$ state. However, because of the hybrid nature of the Poincare map an unique function between the normalized boundary coordinate and the inter-switching time $\Delta t$ as described in Sec.4.3.3 for systems without set-up times does not exist. 
From the above considerations it becomes clear, that even slight changes to a hybrid model (like the introduction of set -up times) may complicate the structure and analysis of the model significantly.Although it results in a similar dynamics. One may argue that a part of these difficulties arises, because we wished to maintain the balanced state during the set-up. But without this requirement a number of other problems is obtained. The modified model with set- up times deserves its attention here mainly due to its natural application for the evaluation of performance of switched server manufacturing systems in the following section.

\subsection{Performance of Manufacturing Systems}

In the foregoing chapter the influence of restricted buffer content and different switching rules on the dynamics of switched server and switched arrival systems was studied. Now we address the question, how different dynamics may affect the performance of manufacturing systems, modeled as hybrid systems.

\subsubsection{Measures of Logistic Performance}

From the viewpoint of production planning and control it is a basic challenge to evaluate the logistic performance of a manufacturing process. The interest in modeling of manufacturing systems concerns not primarily the dynamics, but the estimation of measures of profitability of the manufacturing process in particularly with regard to lead time, due date performance and machinery utilization.

Utilization $(\rho)$ means the ratio of work which is actually done by a production system to its theoretical maximum performance (capacity). The maximum performance of a server is the maximum rate at which it is able to empty (to fill) a buffer, in normalized models given by $\mu=1(\lambda=1)$. Practically the utilization $\rho$ becomes smaller than one if any disturbance reduces the actual production, as for instance during the set-up time. Thus real production systems, where never an absolute lossless production can be guaranteed, always obtain $\rho<1$. However, one of the often contradicting aims of production planning and control is to keep the utilization as high as possible.

The second family of performance measures in production is related to the throughput time $T$, given by the time difference between the entry time of a workpiece and its departure time, and the work in process (WIP, inventory). Whereas a short throughput time saves a quick completion of costumer orders a small level of WIP keeps the capital, bounded in the manufacturing system in form of material, low. The latter variables are connected through Little's Law for the mean values of $T$ (throughput time), $x$ (WIP) and $\lambda$ (arrival rate):

$$
\langle\lambda\rangle=\langle x\rangle\langle T\rangle .
$$


This fundamental equation applies to all systems, regardless if they are stochastic or not [69]. Since these measures are the data usually ascertained in real manufacturing processes we give a number of examples for distributions of throughput times generated by deterministic models. Therewith it should be demonstrated that even pure deterministic systems can show effects that may be similar to stochastic queueing systems, at least at superficially inspection.

In a practical view chaotic behavior, induced by small tank capacities for instance, has two major consequences. On the one hand, the temporal evolution of tank (buffer) contents is more difficult to predict in a chaotic system. On a statistical level this spreads the distributions of inter-switching and throughput times and makes in general the sequence of different products at the departure point irregular. Thus the production planning may be affected. The performance of manufacturing itself is influenced by the number of switchings per time interval for instance which strongly depends on the dynamics.

\subsubsection{The Costs of Switchings}

For models without explicit production losses due to set-up times we study the performance through cost functions. In the context of manufacturing two types of cost functions have to be considered. Usually the set-up of buffer capacities causes costs. For simplicity we assume that these costs are proportional to the maximum buffer capacity and call them buffer cost:

$$
k_{x}=c b
$$

where $c$ is a constant. On the other hand consider the switching cost function:

$$
k_{n}=\lim _{T \rightarrow \infty} \frac{N_{T}}{T} .
$$

where $N_{T}$ is the number of switches in the time interval $T$. Here we assume that every switching of the server causes fixed costs. The dependence of the cost functions on the maximum buffer content $b$ for switched server systems is depicted in Fig. 5.7.

For models designed according to Sec.5.2 the production loss due to switchings can be calculated directly. We consider the production rate:

$$
\rho=\lim _{T \rightarrow \infty} \frac{\int_{0}^{T} d t \mu(t)}{\int_{0}^{T} d t \mu_{0}}
$$

where $\mu(t)=\sum_{i} \lambda_{i}(t)$ is the actual emptying rate while $\mu_{0}=1$ due to the normalization. Fig. 5.8 provides some examples of production rates for switched server systems depending on the maximum buffer capacity $b$. 

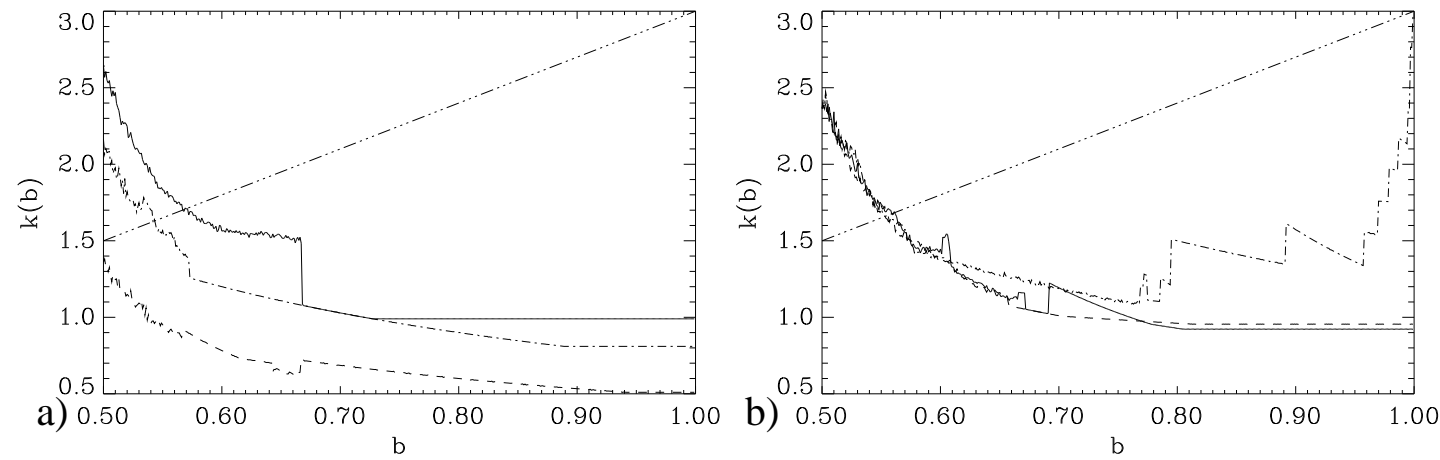

Figure 5.7: Cost functions for three tank switched server systems vs. maximum buffer content $b$. The buffer costs for $c=3$ are given by the three-dot-dashed line. a) The solid curve gives the switching costs for $\lambda_{1}=0.3, \lambda_{1}=0.4, \lambda_{1}=0.3$. The dot dashed curve gives the switching costs for $\lambda_{1}=$ $1, \lambda_{2}=0.8, \lambda_{3}=0.1$ and the dot-dashed curve for $\lambda_{1}=0.1, \lambda_{2}=0.6, \lambda_{3}=0.3$. Compare the bifurcation diagrams given in Fig.4.5.

b) Switching costs for a three tank switched server system $\left(\lambda_{1}=0.45, \lambda_{1}=0.15, \lambda_{1}=0.4\right)$ under different switching rules. Solid line: a cyclic switching rule (rule4.2) is applied, dashed line: rule 4.6 is applied, dot-dashed line: rule 4.7 is applied. Compare the bifurcation diagrams given in Fig. 4.5 and Fig.4.11.
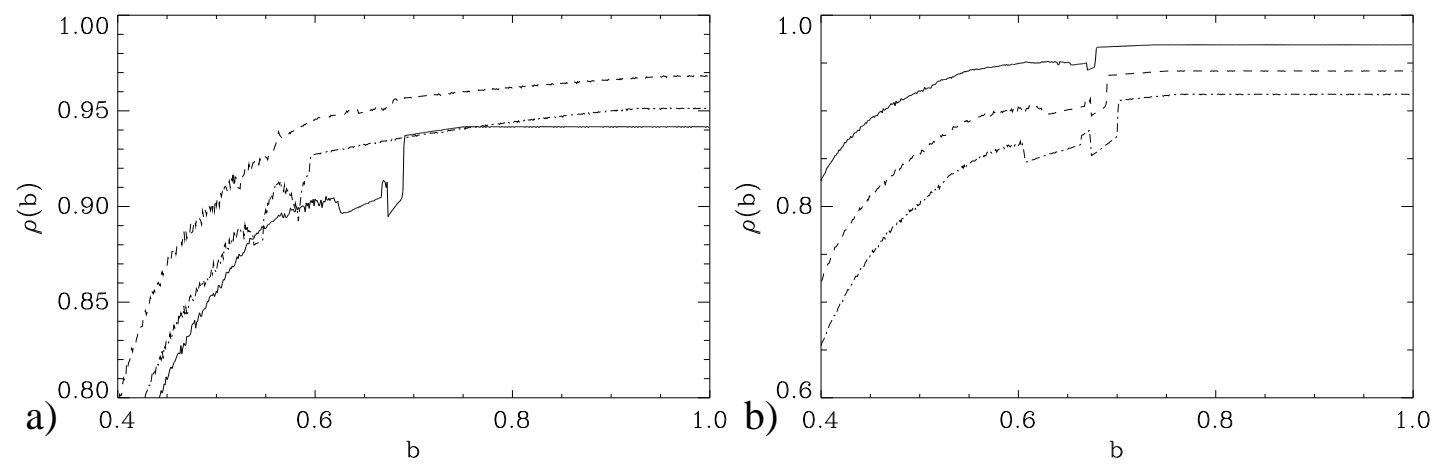

Figure 5.8: Production rate $\rho(b)$ vs. maximum buffer content shows production losses due to set-up times for three tank switched server systems operated with rule 5.1. Note that a lossless production will result in $\rho=1.0$. a) $\rho(b)$ for different parameters and a set-up time $\tau=0.2$. The solid curve gives $\rho(b)$ for $\lambda_{1}=0.3, \lambda_{1}=0.4, \lambda_{1}=0.3$. The dashed curve is for $\lambda_{1}=1, \lambda_{2}=0.8, \lambda_{3}=0.1$ and the dot-dashed curve for $\lambda_{1}=0.1, \lambda_{2}=0.6, \lambda_{3}=0.3$.

b) Production rate $\rho(b)$ for a three tank switched server system with $\lambda_{1}=\lambda_{3}=0.3, \lambda_{2}=0.4$ if different set-up times are applied. Solid curve: $\tau=0.1$, dashed curve: $\tau=0.2$ and dot-dashed curve: $\tau=0.3$.

Every attractor of the system has its own switching costs and production rate, respectively. Therefore discontinuities in the switching cost function and production rate are typical if the system changes the attractor. The switching costs become high (and the production rate shrinks) if the attractor comes close to vertices, because there 
the time interval to the next switching is short. It is obvious in the examples that the chaotic behavior for small $b$ reduce the performance of switched server systems.

Since it is common in the community of industrial engineers to consider "characteristic curves", the curves in Fig.5.8 may be regarded as such. But these curves are characteristics for special deterministic systems with buffers of maximum size $b$ and should be not confused with the $\rho$ vs. $\langle b\rangle$ (here $\langle b\rangle$ is the mean queue length) curves usually applied in production planning [82] that have a similar shape.

\subsubsection{Throughput Times and their Distribution}

The second important measure of logistic performance are throughput time and their distribution. In practical regards it is easy to determine the throughput time of a workpiece since only the time difference of the moment, where the part enters a manufacturing systems and the moment when it departures have to be measured.

For hybrid models with either a continuous arrival process (switched server systems with fixed arrival rates $\lambda_{i}$ ) or a continuous departure process (switched arrival systems with fixed departure rates $\mu_{i}$ ) the throughput time $T$ can be computed directly for all differential amounts of material. In the switched server system ${ }^{4}$ the time $T(t)$ that the material has spent in the buffer up to the moment where it departures is given by:

$$
T(t)=\frac{x_{i}(t)}{\lambda_{i}}
$$

Here $i$ labels the buffer that is actually emptied and $x_{i}(t)$ is its actual filling level. A explanation of this relation is provided by Fig.5.9. Due to the continuous arrival process the entry time is proportional to the actual filling level. The throughput time $T(t)$ thus is a piecewise decreasing function of $t$ in emptying intervals for the specified buffers.

Since in switched server (and switched arrival) systems without set-up times both the initial content $x_{i}$ at the beginning of an emptying interval as well as the duration of the emptying itself are uniquely determined by the normalized boundary coordinate $X$ (see Sec. 4.3) there exists a unique mapping $p(T)=f(T, X)$ where $f(T, X)$ for $X$ fixed is $p(T)=$ const. if $T \in\left(T_{\min }, T_{\max }\right)$ and $p(T)=0$ otherwise. $T_{\min }$ is the throughput time at the end of the associated emptying interval and $T_{\max }$ the throughput

\footnotetext{
${ }^{4}$ In a similar way for the switched arrival systems the throughput time can be determined in the moment, when material enters one of the parallel buffers by :

$$
T(t)=\frac{x_{i}(t)}{\mu_{i}} .
$$

Now $i$ labels the just filled buffer and $x_{i}(t)$ is the actual filling level. The throughput time function now is peace-wise increasing in the intervals of filling.
} 


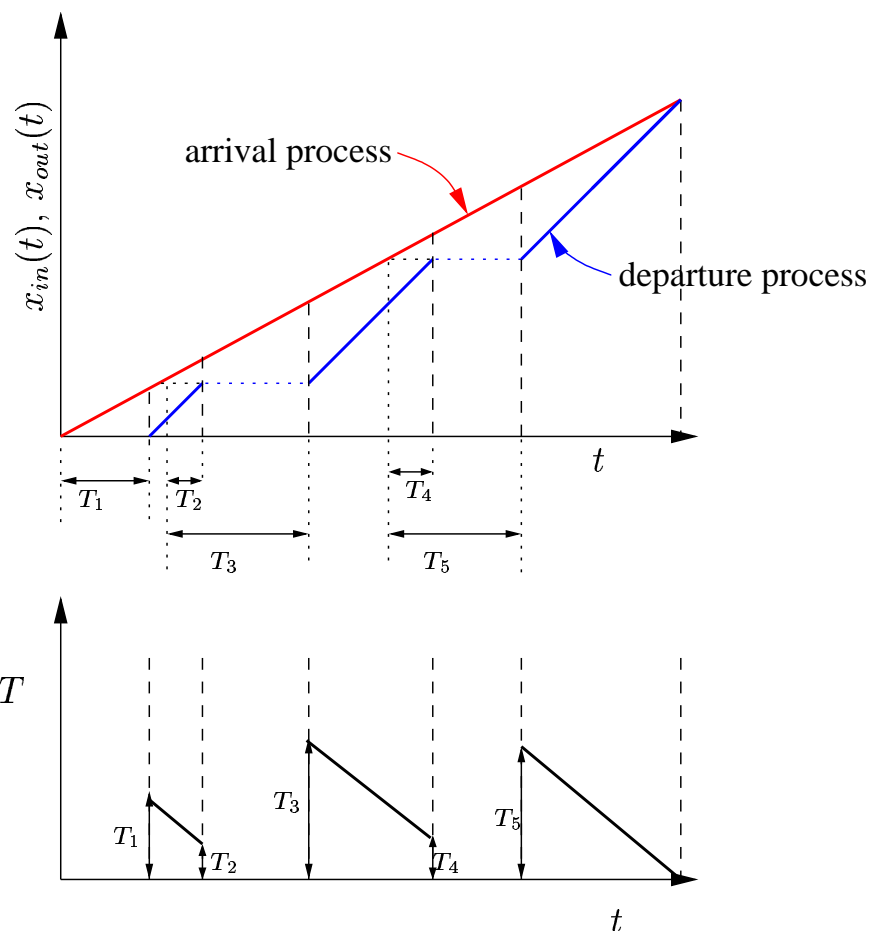

Figure 5.9: On the estimation of throughput times in a hybrid model for switched server systems. The times that the "material" has spent in the buffer is read in the upper part of the figure with separately drawn time evolutions of the cumulative arrivals $\left(x_{i n}\right)$ and departures $\left(x_{\text {out }}\right)$ as function of $t$. The WIP is $x(t)=x_{\text {in }}(t)-x_{\text {out }}(t)$.

time at the beginning of the associated emptying interval. With these relations we can obtain the probability density function of throughput times $p^{*}(T)$ analytically by

$$
p^{*}(T)=n \int_{0}^{1} \mathrm{~d} X p(X) f(T, X)
$$

(where $n$ is a normalizing factor to obtain $1=\int_{0}^{\infty} \mathrm{d} T p(T)$ ) if the invariant probability density $p(X)$ for $X$ is known.

Thus, periodic orbits lead to a piecewise constant probability density functions of throughput times. A piecewise constant invariant density of the orbit, which is obtained in the chaotic limit case for the switched server or switched arrival system, results in a triangular shaped (sawtooth like) probability density function of throughput times (cf. Fig.5.10, first column). If the maximum buffer capacity $b$ in a switched server system is smaller than 0.5 all buffers are never emptied completely. In that case all throughput times are longer than a minimal value larger than zero.

The distinction between throughput time distributions of chaotic and periodic or- 

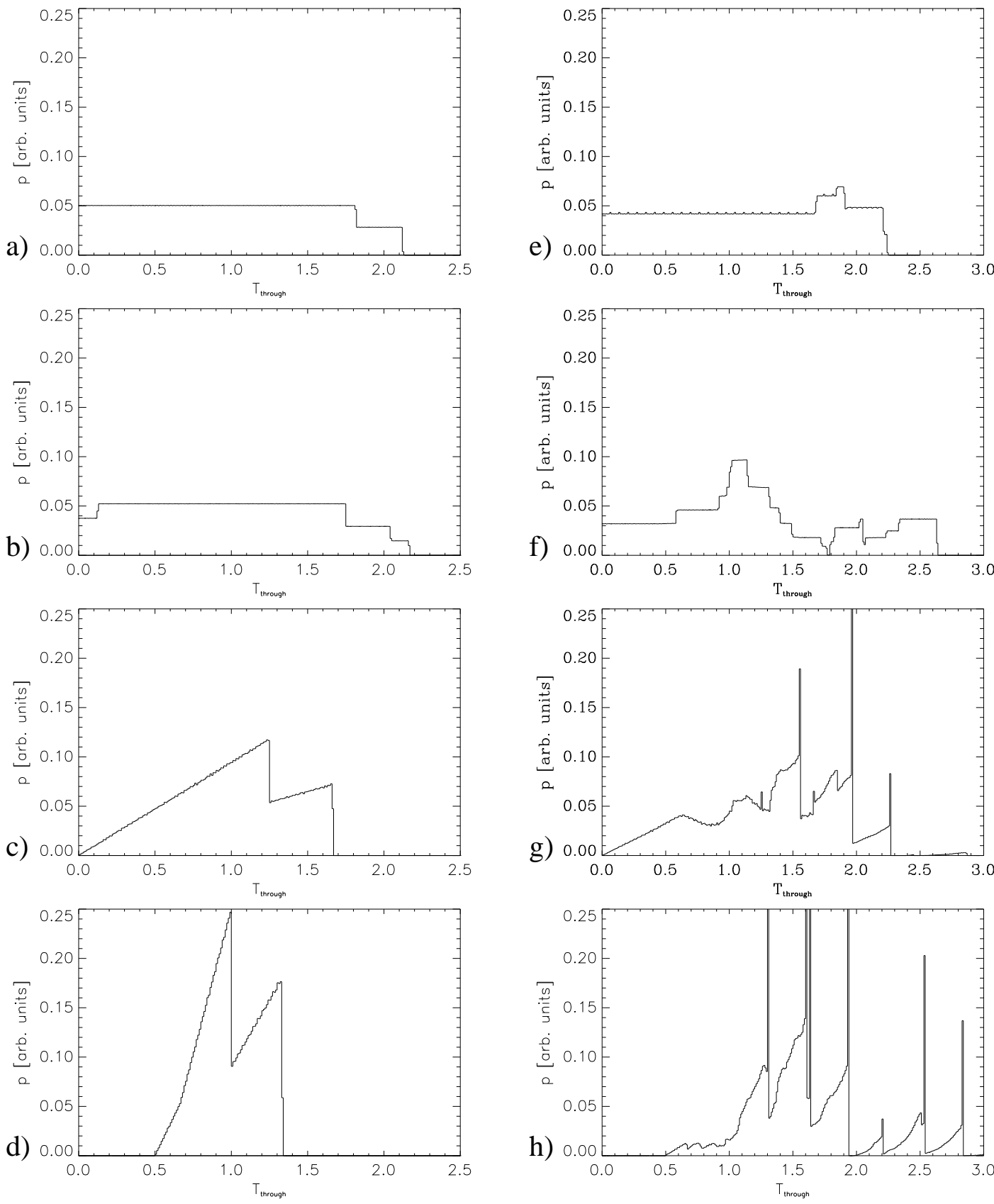

Figure 5.10: Numerically estimated distributions of throughput times in hybrid models for three tank switched server systems, depending on the maximum buffer content for different parameters.

Filling rates: $\lambda_{1}=\lambda_{3}=0.3, \lambda_{2}=0.4$. The first column shows histograms for the system without set-up time, right column histograms for the system with set-up time $\tau=0.3$. The maximum buffer capacity is reduced from top down: a),e) $b=1.0, \mathrm{~b}$ ),f) $b=0.7, \mathrm{c}), \mathrm{g}) b=0.5, \mathrm{~d}), \mathrm{h}$ ) $b=0.4$, 

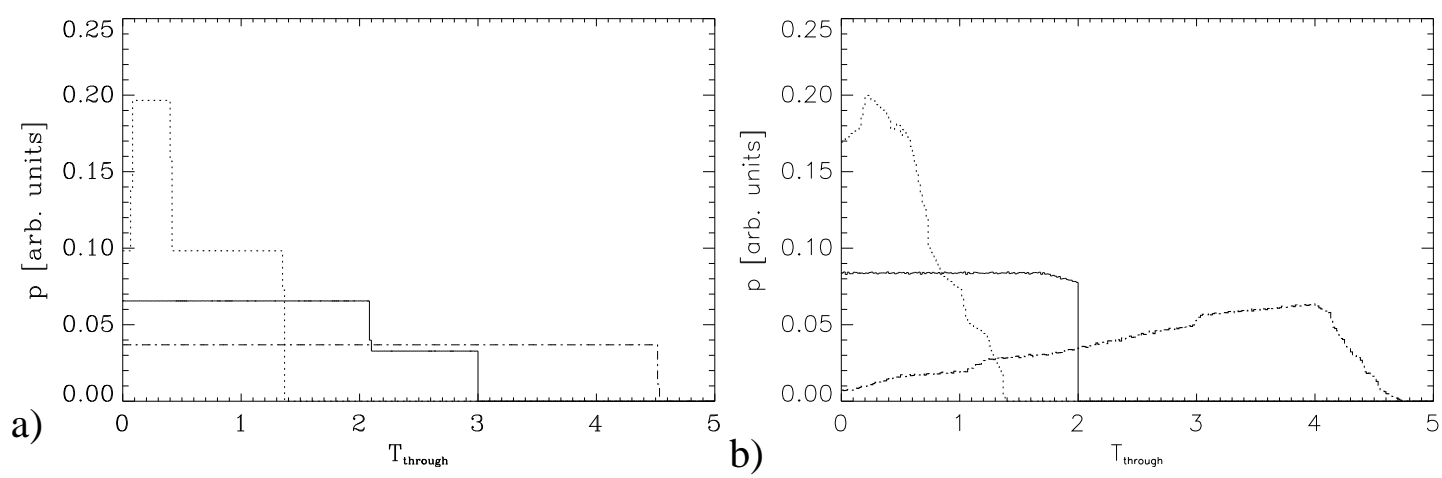

Figure 5.11: Numerically estimated distributions of throughput times in continuous hybrid models for three tank switched server systems, with an upper threshold assigned to the first buffer only.

The solid curve stands for the first (restricted) buffer, the dotted for the second buffer and the dash dotted curve gives the distribution for the third buffer.

a) Filling rates: $\lambda_{1}=\lambda_{3}=0.3, \lambda_{2}=0.4$. The system is not chaotic.

b) Filling rates: $\lambda_{1}=0.1, \lambda_{2}=0.7, \lambda_{3}=0.2$ for $b_{1}=0.2$. The system is chaotic, but the dynamics of the first buffer is periodic.

bits is especially obvious in the histograms shown in Fig.5.11b where the switched server system with an upper threshold at the first buffer only (Sec.4.4.2) was used. Here the throughput time is considered for the different buffers separately. The first buffer exhibits a periodic evolution (of period one) and provides a piecewise constant probability density of throughput times in contrast to the chaotic evolution of the other two buffers.

If systems with a setup time according to Sec.5.2 are considered, the additional time intervals where the filling levels of a buffer are maintained constant must be taken into consideration. In this case an estimation in form of Eq.(5.7) is not possible. A numerical estimation of throughput times shows that the set-up time changes the throughput time distribution compared to the system without set-up (see Fig.5.10). The times during which two of the three buffers are out of service (i.e. the filling level of the remaining buffer is kept constant) lead to exactly the same throughput time for all material that enters and leaves the buffer during this time interval. Therefore some throughput times occur very frequently.

Finally we shall consider the mean throughput time. For switched server systems without set-up times the mean throughput time for the whole system is independent of the parameter $b$ and constant. This follows directly from Little's law (Eq.5.2) because both WIP (total content) and total arrival (departure) rate are constant. For systems with a switching rule according to Sec.5.2 the mean throughput time depends on the utilization and increases for small buffer capacities. 

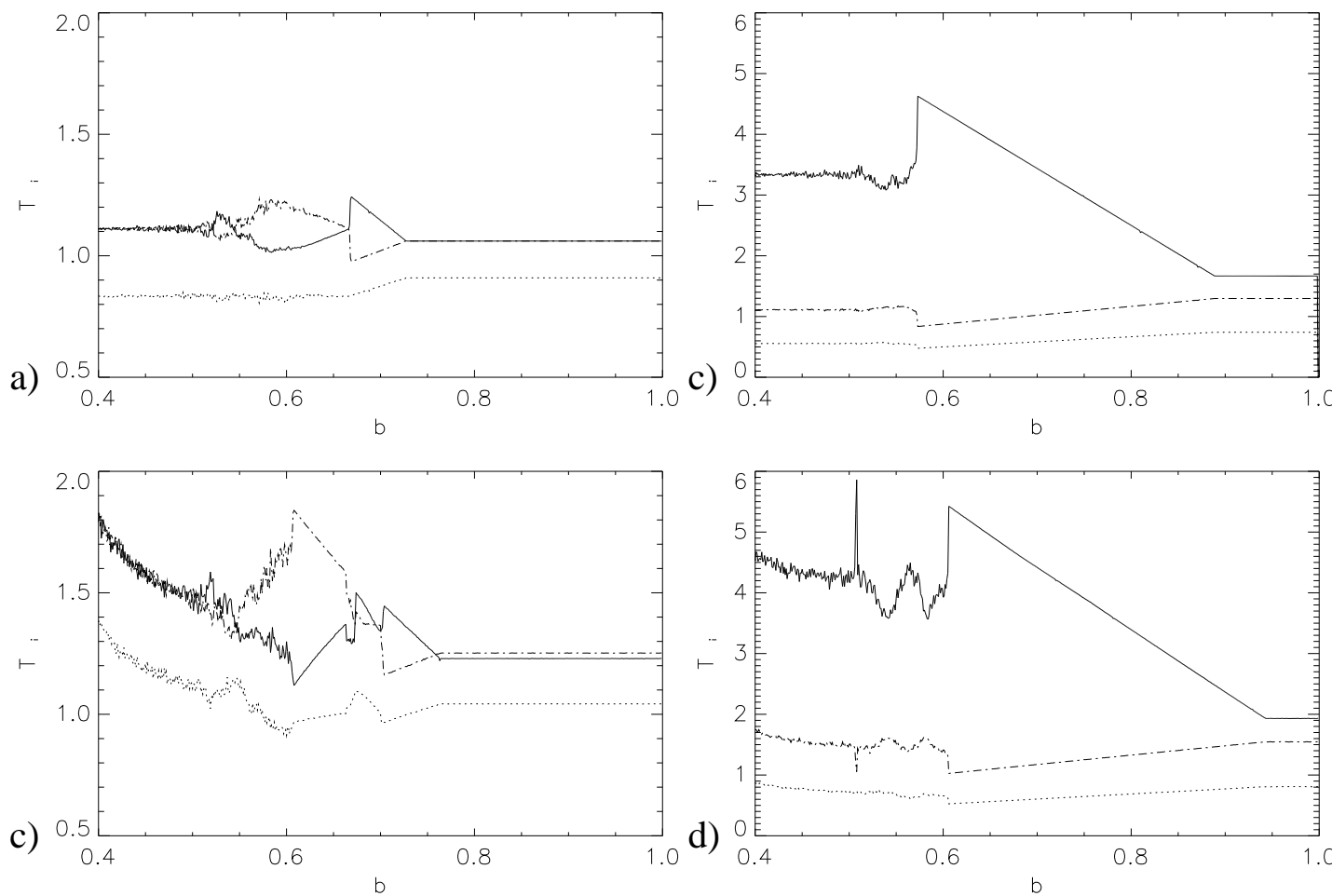

Figure 5.12: Numerical estimation of mean throughput times in a continuous hybrid model for three tank switched server systems. The mean throughput time per buffer vs. maximum buffer content $b$ is shown for different parameters. The solid curves correspond to buffer 1 , the dotted curves to buffer 2 and the dot dashed curves correspond to buffer 3 .

a) $\lambda_{1}=0.3, \lambda_{2}=0.4, \lambda_{2}=0.5$, without any switching time.

b) $\lambda_{1}=0.1, \lambda_{2}=0.6, \lambda_{3}=0.3$, without any switching time.

c) $\lambda_{1}=0.3, \lambda_{2}=0.4, \lambda_{2}=0.3$, a set-up time of $\tau=0.3$ is applied.

d) $\lambda_{1}=0.1, \lambda_{2}=0.6, \lambda_{3}=0.3$, a set-up time of $\tau=0.3$ is applied.

However, the mean throughput times for different buffers may deviate significantly from one another (see Fig.5.12). Moreover, this mean throughput times for individual buffers in the system depend on the attractors of the system and may change with maximum buffer capacity $b$ and or modifyied switching rules (Sec.4.4.1).

\subsubsection{Optimization and Chaos Control}

It is common to reduce buffer costs by lowering $b$. In the switched arrival system this can also prevent chaotic behavior but it induces chaos connected with high switching costs or production losses in the switched server system.

Optimization may therefore address organizational tasks to adjust parameter combinations in a desired range. This can include appropriately chosen switching thresholds or filling rates. Optimization may alternatively consider appropriately chosen switching 
rules for the lower (upper) threshold in the switched server (arrival) system. As we have seen the dynamics is influenced strongly by the switching rule and another rule may lead to stable orbits with lower costs. The advantage of such organizational tasks is, that the system can follow its own dynamics once the parameters are adjusted to the desired values.

Another way may be active control of the dynamics. To optimize both cost functions for the switched server system the maximum buffer content $b$ can be reduced, and then chaos control methods can be applied in the chaotic region to reduce the switching costs.

\subsection{Switched Discrete Deterministic Systems}

The hybrid modeling of manufacturing systems in connection with the strange billiard concept is a powerful tool to evaluate the potential dynamical features of a given topology of manufacturing systems under priority rules and to obtain analytical results in the language of nonlinear dynamics. However, to meet manufacturing reality we have to consider a discrete material flow instead of the fluid modeling presented in the previous sections.

Therefore we now dismiss the approximation of continuous material flows and study the behavior of the previously investigated systems under the assumption of deterministic, but discrete flow of parts. We assume fixed inter-arrival times $(T)$ and inter-departure times $(\Theta)$ for the buffers and servers. The system is balanced if $1 / \Theta=\sum 1 / T_{i}$. The switching rules with respect to the buffer filling levels under consideration should be the same rules as in Sec.4. The main difference between both types of modeling is that a change in the servers position now can occur only at specific times, i.e. the processing of a part is finished. A normalization as with the hybrid models is not feasible in the discrete case. Rather we have to start with an initial buffer content $x_{i} \in \mathbb{N}$ and even discrete thresholds for the buffer capacities. Additionally the initial condition defines the time to the next event inside the intervals, given by the inter-event times. In some sense hybrid models are the limit case of discrete models with infinite total content or infinitesimal inter-event times. Following the terminology of (stochastic) queueing theory such models should be described as deterministic queueing models. In principle the same system of queues, servers and routers as in queueing theory are considered, but instead of stochastic processes the systems are governed by deterministic point processes. 
a)

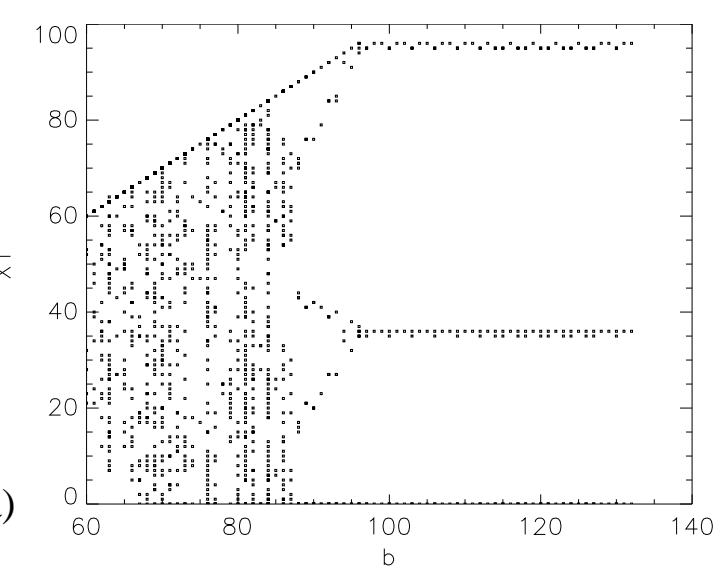

c)
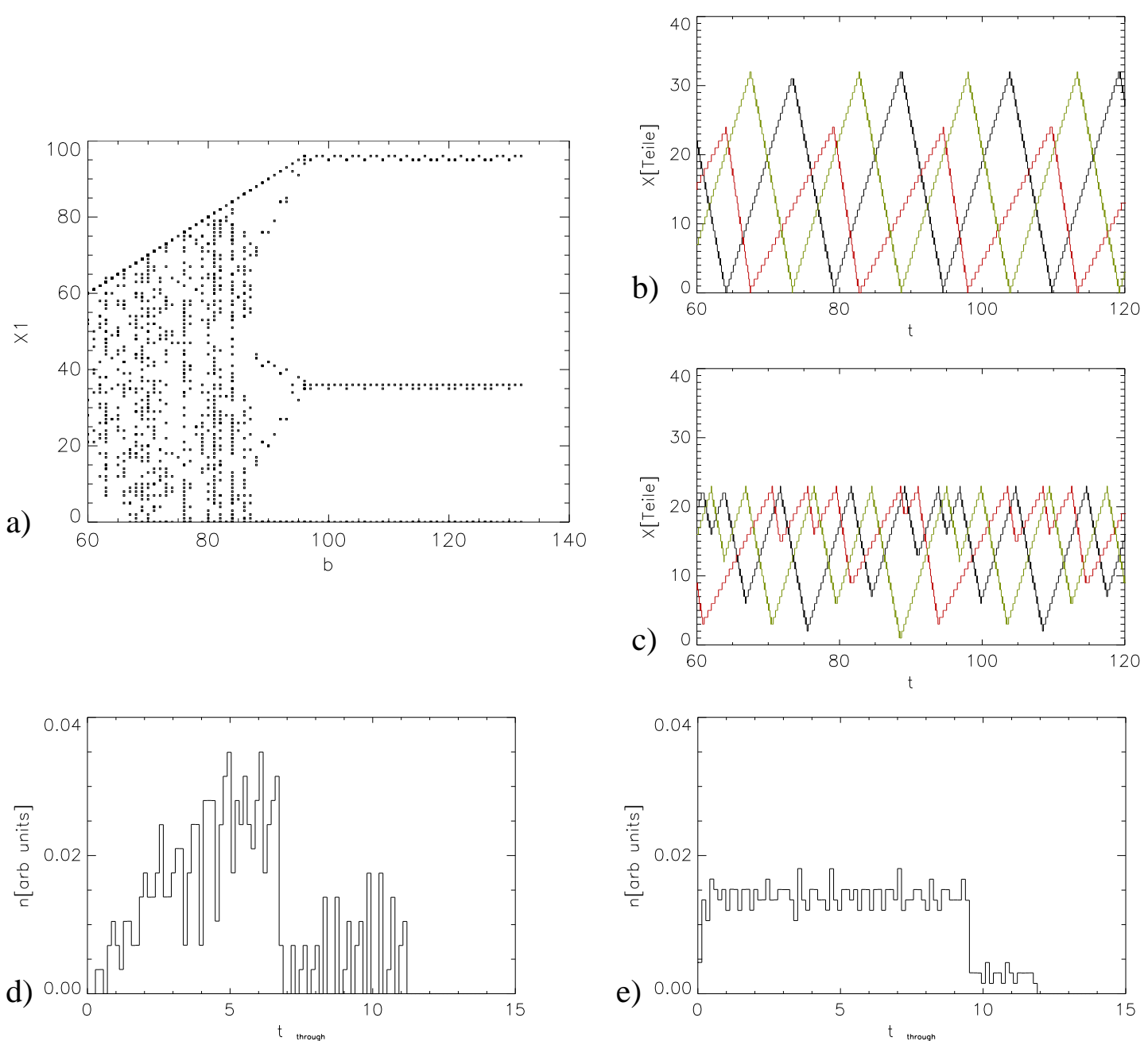

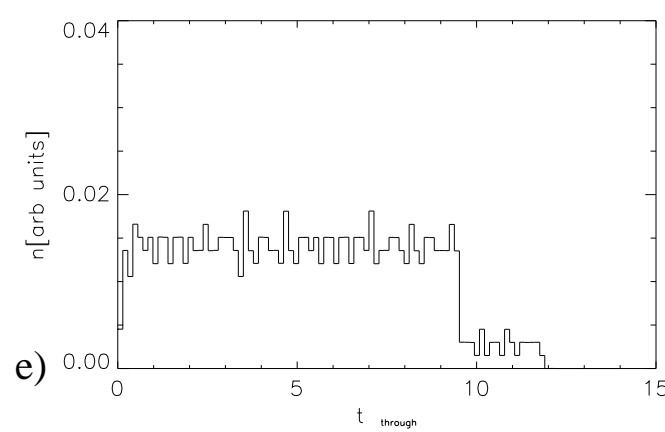

Figure 5.13: Bifurcation like behavior and transition to quasi-chaotic states in a deterministic queuing model of switched server type with three buffers. Parameters: $T_{1}=0.3, T_{2}=0.5, T_{3}=0.3, \Theta=$ 0.11538462 . a) Bifurcation diagram showing the content of the first buffer (measured in parts) at switching times of the server vs. the maximum buffer capacity. Total content: $132 \pm 1$

b),c) Time series of buffer content for a total content of $42 \pm 1$ parts, b) maximum buffer capacity $b=40$, c) maximum buffer capacity $b=23$.

d),e) Histograms of throughput times for the whole system with a total content of $42 \pm 1$ parts for different maximum buffer capacities. d) $b=23$, e) $b=40$.

\subsubsection{Dynamics}

Due to the doubly discrete nature of the system (in ampltude and time) the dynamics of the discrete model is fundamentally distinct to the continuous hybrid model. The discreteness implies a countable, finite number of possible states (filling levels of buffers) and therewith a periodic behavior of the trajectory. Usually a larger number of coexisting orbits in the discrete state space are obtained. Nonetheless the discrete 
model shows in some sense a similar dynamic as the hybrid model even if the transition from the continuous to the discrete material flow fundamentally changes the modeling assumptions in a mathematical framework.

In particular a correspondence of similar coarse grained dynamics in certain parameter regions is obtained. And even in deterministic queuing models bifurcation like phenomena arise (cf. Fig. 5.13a). Small buffer capacities in the switched server system lead to a complicated behavior which may be dubbed quasi-chaotic. For the discrete model it is possible to obtain throughput times for single parts. The distribution of throughput times is determined by the dynamic behavior, and therefore also this logistic parameter depends on the maximum buffer content (Fig. 5.13d,e).

\subsubsection{Small Stochastic Disturbances}

As a further step towards fully realistic modeling of manufacturing we carried out numerical simulations of deterministic queueing models with small stochastic disturbances of inter-event times as they are typical for highly automated manufacturing systems. The results indicate, that such small disturbances mainly lead to slow changes in the total content of the systems. Thus the dynamics is reasonably good approximated by the deterministic queueing models for short time scales. At larger time intervals the system may (because of the varying total content) slowly drift through different dynamics of the pure deterministic model. This leads, for instance, to unexpected chaotic behavior, if the total content of the systems rises through a certain value.

\subsection{Summary}

We have analyzed the dynamical behavior of manufacturing systems governed by the interaction of scheduling policies and limited buffer capacities in the deterministic limit case.

By estimation of cost functions or calculation of production losses due to set-up times it became obvious, that dynamical behavior directly influences the performance of manufacturing systems.

Despite of their relative simplicity the systems investigated here exhibit a complex dynamics, even without irregular influences. Furthermore not only the policies themselves contribute to this behavior, but also the chosen thresholds are crucial where these policies become active. For larger networks of work units with even more rules, thresholds and a huge number of possible discrete states exists. Therefore a highly complex behavior is expected. The transition from a continuous flow to a discrete part flow, which we briefly discussed in Sec.5.2 still remains an open fundamental question. The systems we have introduced here might serve as model systems for the study of such transitions. 
Last but not least the investigations may be seen as a contribution to the discussion on chaos in manufacturing systems $([13,104,67,89,91])$. Whereas in an informal manner the word $\operatorname{chaos}^{5}$ is frequently used by engineers trying to operate manufacturing systems not many sources of a behavior that deserves this term in its scientific sense are identified in manufacturing. The analyse presented here shows, that the intrinsic dynamic of a switched server or switched arrival configurations, depending on the dimension of buffer capacities, can lead to a complex dynamical behavior. This complex behavior is also obtained, if the material flow is discrete. The interdependencies outlined here may be useful to prevent situations in manufacturing where a planning and prediction of production is complicated and the performance of the manufacturing system is reduced.

\footnotetext{
${ }^{5}$ More recently even the term turbulence was used as a metaphor for things going wrong in manufacturing [104][105]. In this setting production engineers try to identify sources of turbulence in manufacturing systems.
} 


\title{
6 Modeling of Front Dynamics in Semiconductor Devices
}

\begin{abstract}
This chapter presents a general hybrid modeling approach for studying front propagation in nonlinear systems with a global constraint. A model of semiconductor superlattices is investigated, where the dynamics of electron accumulation and depletion fronts shows complex spatio temporal patterns.

After a brief introduction into the investigated solid state system (Sec.6.1) a generic hybrid model belonging to the switched arrival class is derived in Sec. 6.2. Then we investigate the dynamics of the model for some cases in more detail.
\end{abstract}

\subsection{Fronts and Pattern Formation in Semiconductor Superlattices and Other Spatio-Temporal Systems}

Moving fronts are the source of complex patterns in very different areas of physics [32, 102], chemistry [61, 76], and biology [93, 78]. While the movement of single fronts is well understood for many systems, not much is known on how front generation and annihilation processes or collisions between fronts influence the possible bifurcation scenarios in systems where fronts of different velocities interact.

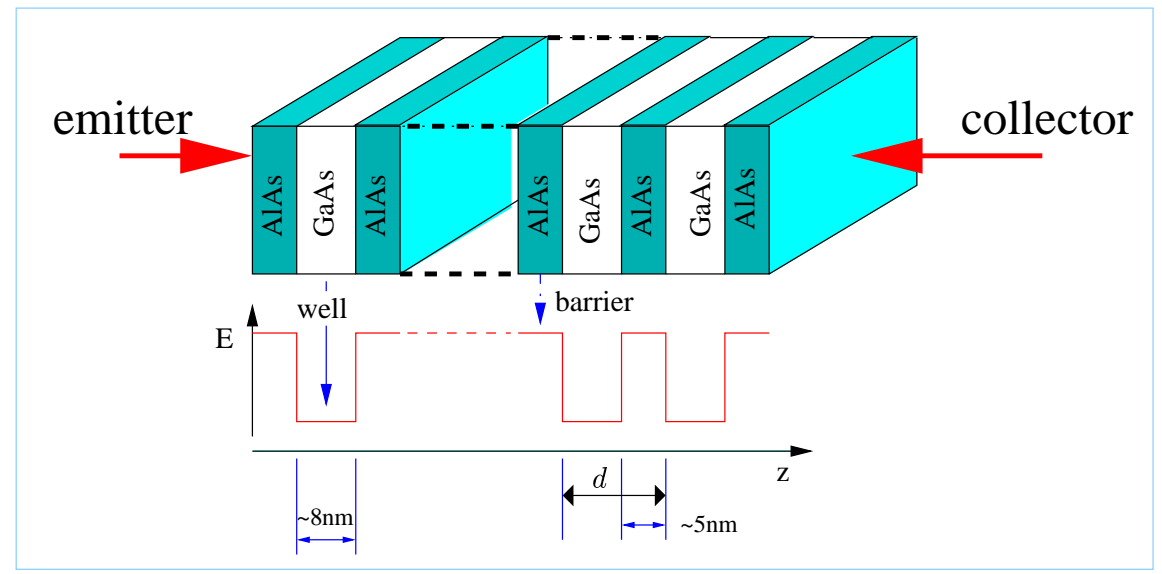

Figure 6.1: Sketch of a typical semiconductor superlattice structure. 
Spatio-temporal pattern formation processes of fronts traveling with different velocities are found, for instance, in semiconductor superlattices. Superlattice structures (cf. Fig.6.1), as suggested in 1970 by Esaki and Tsu [47, 46] are artificial crystal like structures with an adjustable period $d$ that are realized by periodically repeated deposition of alternate layers of different semiconductor materials. The two materials, AlAs and GaAs for instance, possess different band gaps, resulting in discontinuities in both the conduction band and the valence band of the superlattice structure. The conduction band is of interest. Since the conduction band in AlAs is higher than in GaAs the layers of AlAs act as a barrier for electrons, whereas the layers of GaAs represent quantum wells. For superlattices with a large barrier width (weakly coupled superlattices) the structure can be approximated by a series of quantum wells in which the electrons are located. For a finite barrier width the wells become coupled to each other by tunneling processes and the main electrical transport for an applied electric field results from resonant tunneling. This leads to a sequential tunneling model for the electron transport. For details on this approach and other microscopic models see [100].

This model describes the tunneling current density $J_{m \rightarrow m+1}\left(F_{m}, n_{m}, n_{m+1}\right)$ from well $m$ to well $m+1$. It depends only on the electron densities $n_{m}$ and $n_{m+1}$ in the wells and the electric field $F_{m}$ between the wells $[100,3,46]$. The resulting typical $N$ shaped current density vs. electric field characteristic is depicted in Fig.6.2.

In a microscopic simulation the electric fields $F_{m}$ are determined by a discrete version of the Poisson equation:

$$
\varepsilon_{r} \varepsilon_{0}\left(F_{m}-F_{m-1}\right)=e\left(n_{m}-N_{D}\right) \quad \text { for } \quad m=1, \cdots, N
$$

where $N_{D}$ is the doping density and $e<0$ the electron charge. The dynamic evolution of electron densities $n_{m}$ is given by the continuity equation:

$$
e \frac{\mathrm{d} n_{m}}{\mathrm{~d} t}=J_{m-1 \rightarrow m}-J_{m \rightarrow m+1} \quad \text { for } \quad m=1, \cdots, N
$$

In the following a superlattice for a fixed applied voltage $U$ is considered, giving rise to the global constraint

$$
U=\sum_{m=0}^{N} F_{m} d .
$$

Crucial for the formation of patterns are the boundary conditions at the emitter and collector of the superlattice device.

$$
J_{0 \rightarrow 1}=\sigma F_{0} \quad \text { and } \quad J_{N \rightarrow N+1}=\sigma F_{N} \frac{n_{N}}{N_{d}}
$$




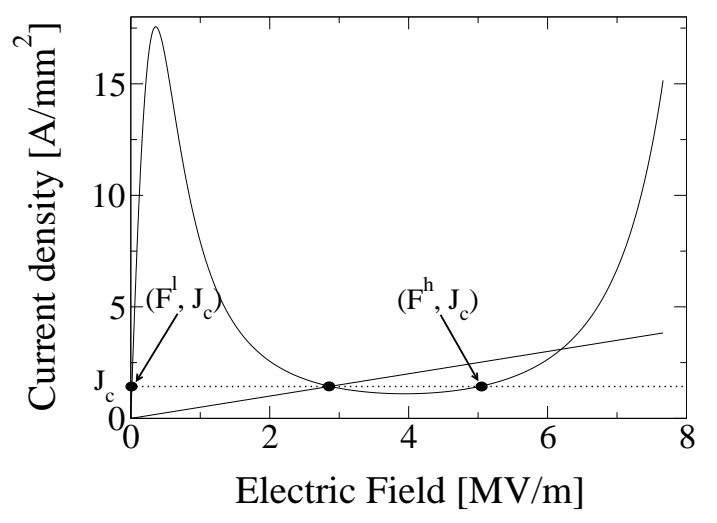

Figure 6.2: $\mathrm{N}$-shaped current density characteristic (current density vs electric field) at the emitter barrier (straight line) and between two neutral wells [3]. The Ohmic conductivity of the emitter is $\sigma=0.5 \Omega^{-1} m^{-1} . J_{c}$ denotes the intersection point of the two characteristics.

where $\sigma$ is the Ohmic conductivity. An N-shaped well-to-well current voltage characteristic with a region of negative differential conductivity has major implications for the behavior of a spatially extended system since it introduces a bistability.

For a given fixed current $J$ a homogeneous electric field for the whole sample is unstable if it falls into the region of negative differential conductivity. This results in the formation of field domains of different local well-to-well electric fields. In the neutral regions of the superlattice, there are two stable points for fixed current $J$, one at a low field $F^{l}(J)$ and one at a high field $F^{h}(J)$ (Fig. 6.2). In the following we make the approximation that both fields do not depend on the current, and $F^{l}=0$ and $F^{h}(J)=F^{h}$. For a transition from a low field domain $F_{m}=0$ to a high field domain $F_{n}=-F^{h}(n>m)$ a negatively charged region (i.e. an electron accumulation front) is required by Poisson's equation. Similarly a transition from a high field domain to a low field domain can be attributed to an electron depletion front. Such fronts can appear only alternating and a high-field domain is located between a leading accumulation and a trailing depletion front. A regime with one electron accumulation and one electron depletion front is called a dipole regime whereas a regime with one depletion and two accumulation fronts or two accumulation fronts and one depletion front is a tripole regime.

For the following considerations it is also helpful to remark, that the velocities of accumulation and depletion front are basically functions of the total current [24, 23, 3]. The relation is depicted in Fig.6.3.

The formation of fronts and field domains due to the negative differential conductivity gives rise to a large menagerie of complex behavior in semiconductor superlattices including self-sustained current oscillations [85], or sawtooth-like currentvoltage characteristics [46, 54, 90]. For a recent review see [100]. While ac driven chaos was already reported some time ago theoretically [21] and experimentally [72], 


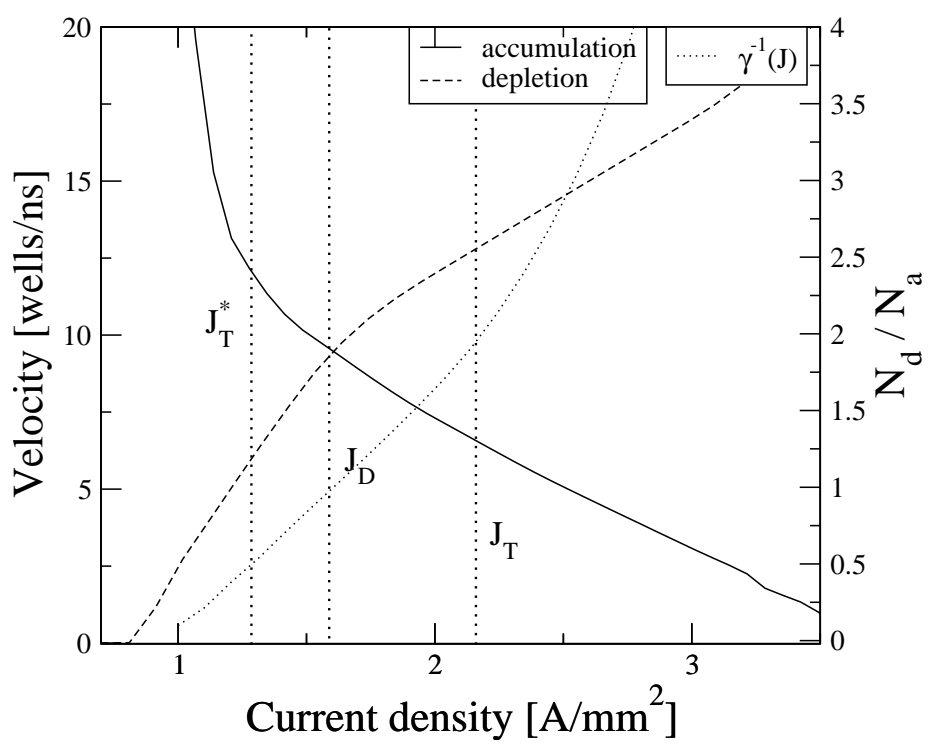

Figure 6.3: Front velocity vs. current density for electron depletion and accumulation fronts [3]. $J_{T}$, $J_{T}^{*}$ denote the currents corresponding to tripole propagation with two accumulation and one depletion front and with two depletion and one accumulation front, respectively. $J_{D}$ is the current corresponding to a dipole propagation with equal velocities for one accumulation and one depletion front. The Ohmic conductivity of the emitter is $\sigma=0.5 \quad \Omega^{-1} m^{-1}$.

autonomous chaos in weakly coupled superlattices was predicted only recently [3]. Depending on the doping density and the contact characteristics the fronts may travel through the lattice with different velocities, annihilate one another and form a complex spatio-temporal pattern under constant voltage conditions.

The main conditions for such behavior are:

1. Boundary conditions that permit multiple stationary states and fronts due to negative differential resistance.

2. An integral over space constraint (e.g.Eq.(6.3)).

These conditions are found in many semiconductor devices [23] and even in molecular electronics $[83,26]$. Patterns which are very similar to the ones we investigate here were found for instance in a spatially continuous semiconductor model describing the impurity impact ionization breakdown in bulk p type Ge [23].

\subsubsection{Benefits of a Generic Model}

The straight forward approach for studying the dynamic behavior of superlattices is to numerically integrate the charge densities $n_{i}(t)$ in each well using a sequential tunneling model. This leads to a system of typically 100 coupled ODEs with well over 15 physically relevant parameters. Over the last decade this method has proven 
to yield quantitative data which could be verified in a large range of experiments [100]. From the dynamical system point of view, it is still highly desirable to find a generic model which captures the essential dynamics and bifurcation scenarios of the full system in only a few dynamical variables and parameters. This is especially important in the case of complex behavior, where the type of complexity should be completely characterized by the generic model.

\subsection{Modeling Front Dynamics}

In the following we motivate a simplified model for the front dynamics, which will lead to a version of the switched arrival hybrid system. Hence a discrete quantum well structure is approximated by a one dimensional continuum.

Let us denote by $a_{i}$ the distance of an electron accumulation front (or more precisely its center of charge) from the emitter. The index $i=1, \ldots, N_{a}$ labels the accumulation fronts depending on their emitter distance, such that $a_{i}<a_{i+1}$ and $N_{a}$ is the number of accumulation fronts. In the same way the positions of depletion fronts are denoted by $d_{i}$ with $i=1, \ldots, N_{d}$, and $N_{d}$ is the number of depletion fronts. Since we only allow for alternating accumulation and depletion fronts, we have $N_{d}-N_{a}=-1,0,1$.

The applied voltage $U$ between emitter and collector defines the total length $L^{h}=$ $U / F^{h}$ of the lattice that is at high field. This imposes a constraint on the fields and at the same time on the front positions by

$$
L^{h}=\sum_{m=0}^{N}-\frac{F_{m}}{F^{h}} \frac{L}{N}=\sum_{i=1}^{N_{d}} d_{i}-\sum_{i=1}^{N_{a}} a_{i} \bmod L .
$$

Here $L$ denotes the total length of the superlattice. The expression $\bmod L$ in (6.5) means that $L$ is to be added as necessary in order for $L_{h}$ to be in the interval $[0, L]$. Note that although $a_{i}$ and $d_{i}$ are continuous variables, Eq. (6.5) is exact even for a discrete lattice with extended fronts, as long as all charge can be uniquely assigned to one front.

By assuming that the front velocities are a function of the total current $[24,5]$ we have $\dot{a}_{i}=v_{a}$ and $\dot{d}_{i}=v_{d}$ for all fronts. Differentiating Eq. (6.5) with respect to time yields

$$
\frac{N_{a}}{v_{d}}=\frac{N_{d}}{v_{a}}
$$

for the relative velocities. We may fix the absolute velocities using $v_{a}+v_{d}=2$ by rescaling time.

In the microscopic model it is found that the front injection at the emitter is governed by a critical current $J_{c}$. For $J<J_{c}$ the region close to the emitter is pinned at a low field [100]. Hence an accumulation front is injected, if the preceeding front is a 
depletion front. Due to the finite width and build-up time of the fronts new fronts can not be injected arbitrarily fast. Phenomenologically we therefore introduce a distance parameter $p^{h}$, and assume that front generation is suppressed while $d_{1}<p^{h}$, where $d_{1}$ is the position of the first depletion front. In the same fashion, a depletion front is injected if $J$ rises above $J_{c}$ and $p^{l}<a_{1}$, where $p^{l}$ is a parameter describing the minimum front distance for depletion front injection.

Since the current $J$ is a monotonous function of $N_{a} / N_{d}$ [3] (cf. Fig.6.3), the parameter $J_{c}$ may be replaced by a new parameter $r_{c}$ and the above condition $J \gtrless J_{c}$ is transformed into $N_{a} / N_{d} \gtrless r_{c}$.

The processes which reduce the number of fronts are collision of two fronts of opposite polarity and the running out of fronts at the emitter. Both processes affect $N_{a} / N_{d}$ and potentially trigger a new front generation at the emitter.

We summarize the front model by the following rules:

(I) Evolve the positions of the fronts linearly according to

$\dot{a}_{i}=\frac{2 N_{d}}{N_{a}+N_{d}} \quad$ for $i=1 \ldots N_{a}$

$\dot{d}_{i}=\frac{2 N_{a}}{N_{a}+N_{d}} \quad$ for $i=1 \ldots N_{d}$

until one of the following rules applies.

(II) If $N_{a} / N_{d}<r_{c}$ and $p_{h}<a_{1}<d_{1}$ then increase $N_{a}$ by one, re-index $a_{i} \rightarrow a_{i+1}$ for all $i$ and set $a_{1}=0$

(III) If $N_{a} / N_{d}>r_{c}$ and $p_{l}<d_{1}<a_{1}$ then increase $N_{d}$ by one, re-index $d_{i} \rightarrow d_{i+1}$ for all $i$ and set $d_{1}=0$

(IV) If $a_{i_{a}}=d_{j_{a}}$ for any $i_{a}, j_{a}$ then decrease $N_{a}$ and $N_{d}$ by one, re-index $a_{i+1} \rightarrow a_{i}$ for $i \geq i_{a}$ and $d_{j+1} \rightarrow d_{j}$ for $j \geq j_{a}$.

(V) If $a_{N_{a}}>L$ decrease $N_{a}$ by one.

(VI) If $d_{N_{d}}>L$ decrease $N_{d}$ by one.

Note that the voltage parameter $L_{h}$ only enters in the starting position for the fronts (see Eq. (6.5)) and that for $r_{c}^{\prime}=r_{c}^{-1}$ the characters of accumulation and depletion 


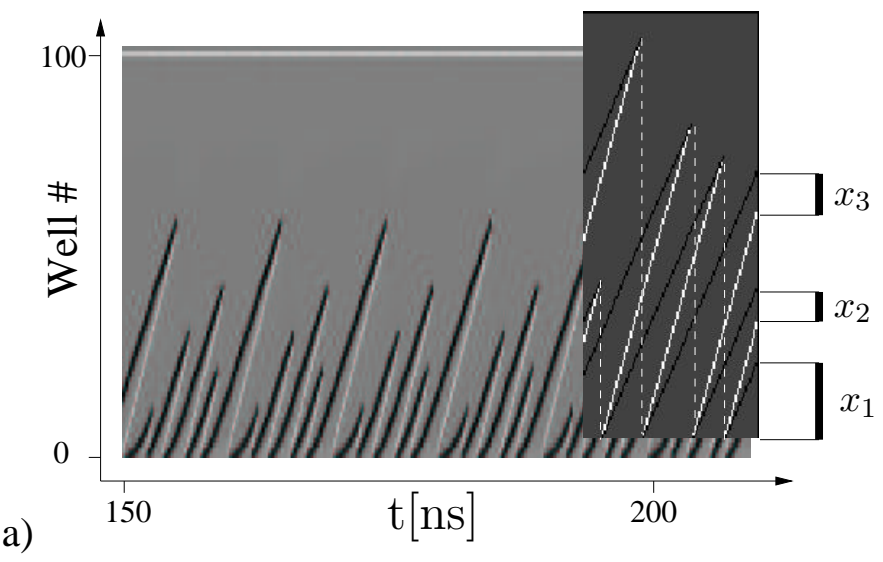

Figure 6.4: Space-time plot of the electron density evolution in a semiconductor superlattice. Electron accumulation and depletion fronts are indicated by light and dark shading, respectively. The full microscopic sequential tunneling model [3] for $n=100$ quantum wells and contact conductivity $\sigma=0.5 \Omega^{-1} m^{-1}$ is used for a bias $U=0.95 \mathrm{~V}$. The inset shows the simplified view of the pattern which is adopted in the hybrid model. The dashed lines denote the switching times $t_{m}$

fronts are interchanged.

In the following we will restrict ourselves to the case $r_{c}<1$ and $p^{l}=0$. All considerations that are made for high-field domains here apply also to low-field domains if $r_{c}>1$. Rule 6.8 does not apply as long as $N_{a}>r_{c} /\left(1-r_{c}\right)$ and $N_{a}$ can only decrease. Consequently $r_{c}$ imposes the following limits on the number of fronts:

$$
N_{a}<\frac{1}{1-r_{c}} \quad N_{d}<\frac{1}{1-r_{c}}+1 \text { for } r_{c}<1
$$

Since the number of high-field domains is given by the number of fronts this implies a maximum number $n$ of high-field domains of:

$$
n=\operatorname{int}\left[\frac{1}{1-r_{c}}+1\right]
$$

where int $[x]$ means the largest integer smaller than $x$. The injection of an accumulation front is immediately followed by the injection of a depletion front (rule 6.9). This detaches a high-field domain from the emitter and opens a new one.

Let us now introduce the length of the high-field domains (see Fig.6.4):

$$
\begin{aligned}
x_{1} & =d_{1} \\
x_{i} & =d_{i}-a_{i-1} \quad \text { for } i=2 \ldots N_{d}
\end{aligned}
$$

Further we require the maximal number of high field domains $n$ for a given $r_{c}$ (6.14). In case no fronts reach the collector, the rules 6.11 and 6.12 do never apply. The case when fronts are able to reach the collector is considered in Sec.6.4. The rules for the front model can be now reformulated in terms of $x_{i}$ by the following rules for a tank 
model:

(1) Filling and emptying rates:

$\dot{x}_{1}=\lambda=\left(N_{d}-1\right) \mu$
$\dot{x}_{i}=-\mu=-\frac{1}{\left(N_{d}-1\right)} \quad$ for $i=2 \ldots N_{d}$

(2) If $x_{i_{a}}=0$ then decrease $N_{d}$ by one, re-index $x_{i+1} \rightarrow x_{i}$ for all $i>i_{a}$ and $x_{i_{a}} \rightarrow x_{\left(n-N_{d}\right)}$.

(3) If $N_{d}<1 /\left(1-r_{c}\right)$ and $x_{1}>p_{h}$ then increase $N_{d}$ by one, re-index $x_{i} \rightarrow x_{i+1}$ for all $i$.

The conservation law (6.5) can be expressed now by

$$
L_{h}=\sum_{k} x_{k}
$$

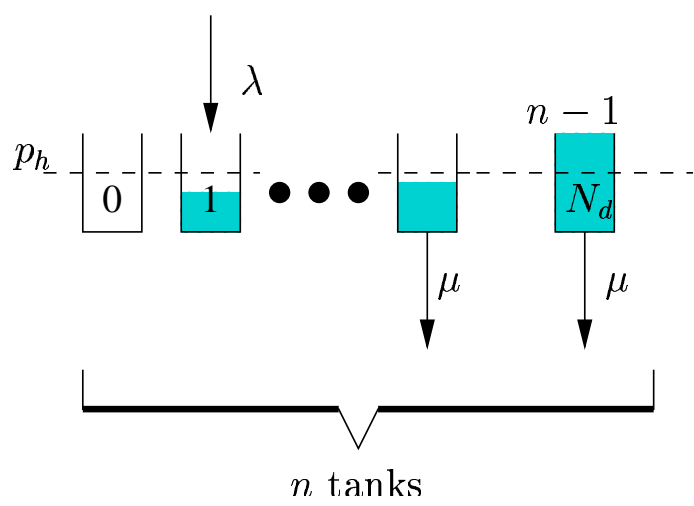

Figure 6.5: Illustration of a typical situation in the tank model with $n$ tanks. The minimum filling level is indicated by $p_{h}$. One tank (indexed by 0 ) is waiting, whereas $N_{d}=n-1$ tanks are emptied or filled and the fronts they are assigned to are present in the superlattice.

It is very illustrative to interpret this model in the language of an $n$-tank switched arrival system (cf. Fig.6.5). A domain may be seen as a tank with fluid content $x_{i}$. Now we have $n$ of such tanks, where one of them is filled at rate $\left(N_{d}-1\right) \mu$, while the others are drained with rate $\mu\left(x_{i} \mid i=1, \ldots, N_{d}\right)$, or waiting for filling $\left(x_{i} \mid i<1\right)$, because the currently filled tank has not reached the minimal filling level $p_{h}$. Apart from the re-indexing procedure and the normalization of the total content to $L_{h}$ (instead 
of one) this configuration is well known from Chap. 4 but here a minimal switching threshold in form of $p_{h}$ is introduced.

In the following we shall investigate the dynamics of this model.

\subsection{Dynamics for Long Superlattices}

In this section we consider the dynamics of the hybrid model under the assumption that no fronts reach the collector. This implies a long superlattice sample. Since no front reaches the collector, the only effect is the annihilation of fronts, which results in the generation of a new dipole at the emitter site. This new dipole contains a depletion front, which opens a new domain at the emitter site growing with a rate $v_{d}$. The previously growing domain will be closed by the accumulation front and shrinks now continuously with the rate $\mu=\left(v_{a}-v_{d}\right)$. If another domain annihilates (i.e. its content reaches zero) the same process repeats.

\subsubsection{Limiting Case of a Vanishing Threshold}

At first we discuss the limiting case $p_{l}=p_{h}=0$. If a tank is empty at time $t_{m}$ $\left(x_{k}\left(t_{m}\right)=0\right)$ the rules 6.18 and rule 6.19 apply instantaneously and the system starts to fill this tank $\left(x_{1}\left(t_{m}^{+}\right)=0\right)$. In this case no indices $i<1$ occur, i.e. no tank has to wait for filling. Apart from the re-indexing procedures we obtain a switched arrival system (Sec.4.2). As shown in [92] the system is chaotic for all $n>2$ and has a constant invariant probability measure.

By sampling the dynamics at the event times $t_{m}$ we obtain the Poincaré map $P$ : $\vec{x}\left(t_{m}\right) \mapsto \vec{x}\left(t_{m+1}\right)$ :

$$
\begin{array}{ll}
\vec{x}\left(t_{m+1}\right) & =\vec{x}\left(t_{m}^{+}\right)+\dot{\vec{x}} \Delta t_{m} \\
\Delta t_{m} & =\min _{j \neq 1}\left(x_{j}\left(t_{m}^{+}\right) / \mu\right)
\end{array}
$$

where $\dot{\vec{x}}=(\lambda,-\mu, \ldots,-\mu)$ is a constant vector and $\Delta t_{m}=t_{m+1}-t_{m}$.

Generally this is an $n$ - dimensional mapping which acts due to the normalization $\sum_{i} x_{i}=1$ on the $n-2$ dimensional boundary of a regular $n$-simplex embedded in $\mathbb{R}^{n}$. The re-indexing procedure can be seen as a rotation of the $n$-simplex which brings the subsimplex $x_{i}=0$ in the position of the $x_{1}=0$ subsimplex. To link the dynamics of the tank model for fronts with the hybrid dynamical systems investigated in the previous chapters we have included some figures (e.g. Fig.6.6), where the well known regular 3-simplex is not rotated and the strange billiard trajectories can be obtained directly.

Since all tanks are indistinguishable with respect to their filling and emptying rates here we can denote the $n-2$ dimensional mapping directly. For $n=3$ the map reduces 


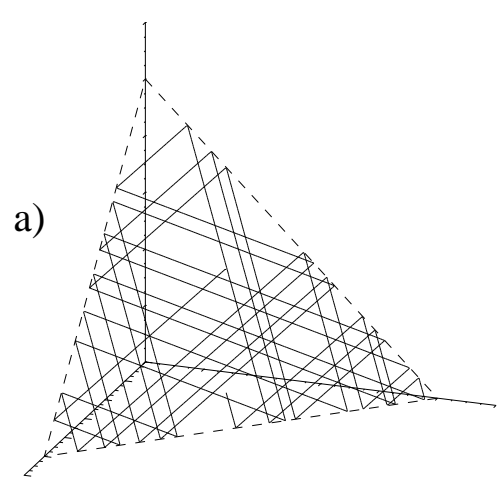

b)

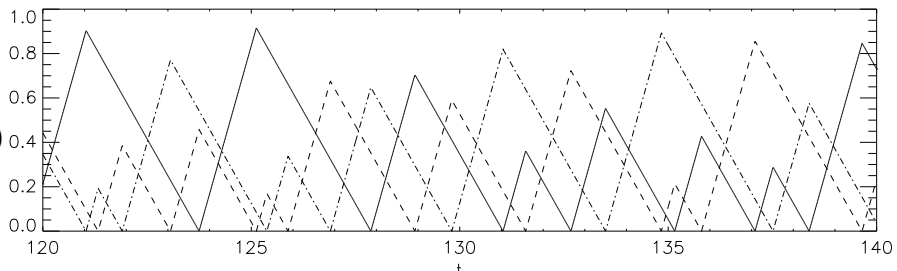

c)

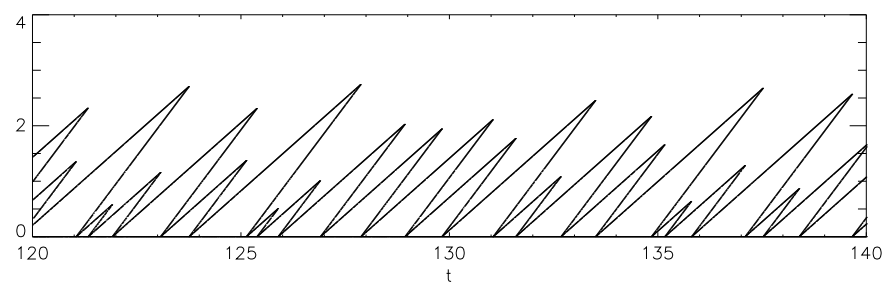

Figure 6.6: Illustration of the basic model for three domains. a) The trajectory of domain sizes moves uniformly inside the 3 -simplex $S_{3}$ embedded in $\mathbb{R}^{3}$. b) Sample evolution of domain contents. The different tanks (domains) are indicated by solid, dashed and dot-dashed lines. c) Associated evolution of fronts on the lattice with normalized velocities.

to a one dimensional map of the interval $\left(0, L_{h}\right)$ onto itself describing successive filling levels of the first tank at $t_{m}$. From the rules we obtain $x_{1}\left(t_{m}^{+}\right)=0, x_{2}\left(t_{m}^{+}\right)=x_{1}\left(t_{m}\right)$, and $x_{3}\left(t_{m}^{+}\right)=L_{h}-x_{1}\left(t_{m}\right)$. If $x_{1}\left(t_{m}\right)<L_{h} / 2$, the tank 2 empties after $\Delta t_{m}=$ $x_{1}\left(t_{m}\right) / \mu$ and we find $x_{1}\left(t_{m+1}\right)=2 x_{1}\left(t_{m}\right)$. Otherwise, if $x_{1}\left(t_{m}\right)>L_{h} / 2$, tank 3 empties after $\Delta t_{m}=\left(L_{h}-x_{1}\left(t_{m}\right)\right) / \mu$ and we find $x_{1}\left(t_{m+1}\right)=2\left(L_{h}-x_{1}\left(t_{m}\right)\right)$. Thus the dynamics is that of the well known tent map:

$$
x_{1}\left(t_{m+1}\right)=L_{h}-\left|2\left(x_{1}\left(t_{m}\right)-L_{h} / 2\right)\right| .
$$

For $n=4$ we consider the filling level of the first tank and the emptiest remaining tank ( $x_{S}$, without loss of generality ) as appropriate variables for the return map. Since either the just filled tank or the tank with content $x_{S}$ is the next empty tank the determination of the filling level at $t=t_{m+1}$ is straightforward. Thus for the construction of the return map only the filling level of the smallest remaining tank at $t=t_{m+1}$ should be determined. Since all filling levels are known we obtain the following map:

$$
\begin{aligned}
& x_{1}\left(t_{m+1}\right)=\min \left\{3 x_{1}\left(t_{m}\right), 3 x_{S}\left(t_{m}\right)\right\} \\
& x_{S}\left(t_{m+1}\right)=\left\{\begin{array}{lll}
x_{S}\left(t_{m}\right)-x_{1}\left(t_{m}\right) & \text { if } & x_{S}\left(t_{m}\right)>x_{1}\left(t_{m}\right) \\
\min \left\{x_{1}\left(t_{m}\right)-x_{S}\left(t_{m}\right), L_{h}-x_{1}\left(t_{m}\right)-2 x_{S}\left(t_{m}\right)\right\} & \text { if } & x_{S}\left(t_{m}\right)<x_{1}\left(t_{m}\right)
\end{array}\right.
\end{aligned}
$$


For all $n>4$ a similar map can be constructed, if $x_{1}\left(t_{m}\right)$ and the $n-3$ smallest remaining tanks are used as variables for the iterated map. For $r_{c} \rightarrow 1$ however the dimension diverges $(n-2) \rightarrow \infty$.

\subsubsection{The Parameter $p_{h}$}

The basic model, considered in the previous allows the opening of a new domain for any size of the previously filled domain. In contrast in semiconductor superlattices the injection of a new dipole at the emitter is suppressed if a depletion front is near the emitter. To include this effect the parameter $p_{h}$ was introduced and assumed that the filling of an empty domain only starts if the previously filled one has a size $x_{i} \geq p_{h}$. If a domain is empty $\left(x_{i}\left(t_{0}\right)=0\right)$ and the currently filled domain $x_{q}\left(t_{0}\right)<p_{h}$ it remains empty until $x_{q}\left(t_{1}\right)=p_{h}$. During this interval only $n^{\prime}=n-1$ domains are present in the system. Due to this fact we have to adjust the empty-rate to $\mu^{*}$ with $\mu_{i}^{*}=1 / n^{\prime}-1$ and the normalized front velocity $\tilde{v}_{d}=1-1 / N^{*}$ for the remaining domains. But if one subsequent tank is empty $x_{i}\left(t_{1}\right)=0 \mid i=1,2$ while $x_{1}\left(t_{1}\right)<x_{\min }$ only rule 6.18 applies and the empty tank is sent to a waiting state (indexed by $i<1$ ). In this state the rates are changed according to rule 6.17. In the strange billiard picture the trajectory moves with a new velocity vector along the boundary $\delta S_{n}$ (see Fig. 6.9 for illustration).

For $n=2$ (i.e. $r_{c} \leq 1 / 2$ ) the minimal switching height has no influence and the pattern is periodic.(If one domain is empty, all current is concentrated in the remaining domain which is over the threshold due to this fact.)

For $n=3$ only one domain can be empty without the currently filled domain is over the threshold. But for $n>3$ we have a possibility to have two or more (up to $n-2$ ) empty domains for which the filling is prevented by the threshold $p_{h}$. That increases the number of discrete states in the hybrid model to $n+(n-2) N$. The corresponding trajectories are restricted to subsimplexes of decreasing dimensions the more tanks are in the waiting state. However, loosely speaking, the system "knows" how many tanks it has due to the parameter $r_{c}$ that is given by physical conditions (i.e. $\sigma, U$, see Sec.6.2).

\subsubsection{Dynamics for Three Domains}

Now we consider the case $p_{l}>0, n=3$. The Poincaré map Eq.(6.22) remains unchanged if $p_{h} / 2 \leq x_{1}\left(t_{m}\right) \leq\left(1-p_{h} / 2\right)$. But if one subsequent tank is empty $\left(x_{i}\left(t_{1}\right)=0 \mid i=1,2\right)$ while $x_{1}\left(t_{1}\right)<p_{h}$ only rule 6.18 applies and the empty tank is sent to a waiting state and the rates are changed according to rule 6.17. Nonetheless, at a later event time $t_{m}$ we get $x_{1}\left(t_{m}\right)=x_{\min }$ and now the filling of the empty tank 
starts. In this case we take $t_{m}$ as sampling time for the map. Thus for $n=3$ we obtain (cf. Fig.6.7a)):

$$
x_{1}\left(t_{m+1}\right)=\max \left\{L_{h}-2\left|x_{1}\left(t_{m}\right)-L_{h} / 2\right|, p_{h}\right\}
$$

The map is depicted in Fig.6.7. Due to the flat segments and the remaining tentmap part we call the map Eq.6.24 flat bottom tent map in the style of [53] where the flat topped tent map appears. For physical reasons the map is not defined for $x<p_{h}$. However, for the long term behavior we may also consider the form given in Eq.(6.24) since every point in $\left(0, p_{h}\right)$ is mapped within one iteration into the invariant set $\left[p_{h}, L_{h}\right)$. For the hybrid model $\Delta t_{m}\left(x_{1}\left(t_{m}\right)\right)$ is a unique function given by:

$$
\begin{aligned}
\Delta t_{m}\left(x_{1}\left(t_{m}\right)\right)= & \max \left\{\frac{\left.L_{h}-2\left|x_{1}\left(t_{m}\right)-L_{h} / 2\right|\right)}{2}\right. \\
& \left.p_{h}-\left(L_{h}-2\left|x_{1}\left(t_{m}\right)-L_{h} / 2\right|\right)+\frac{\left.L_{h}-2\left|x_{1}\left(t_{m}\right)-L_{h} / 2\right|\right)}{2}\right\}
\end{aligned}
$$
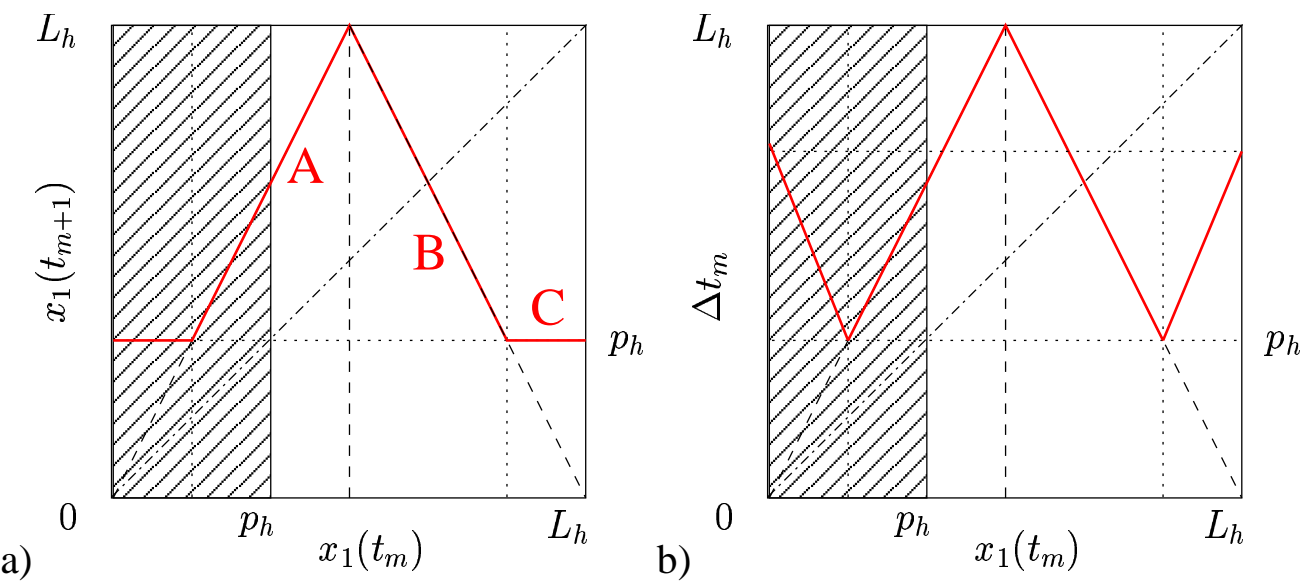

Figure 6.7: Illustration of the return map for three domains. a) The modified tent map (6.24) b) The graph of function Eq.(6.25) giving $\Delta t_{m}\left(x_{1}\left(t_{m}\right)\right)$

The introduction of a minimal switching threshold fundamentally changes the dynamics of the model compared to the chaotic limiting case discussed in Sec.6.3.1. A bifurcation diagram for map 6.24 is given in Fig.6.8. It is convenient to plot this diagram using $x_{1} / p_{h}$ vs. $L_{h} / p h$ for two reasons. On the one hand consider the superlattice. Owing to $L_{h} U$, the axis $L_{h} / p_{h}$ reads the voltage applied to the superlattice (assumed that $p_{h}=$ const. for the superlattice system). Furthermore, $x_{1} / p_{h}$ gives a value proportional to the distance of the leading depletion front in the lattice, i.e. the initial domain content (at the moment where the domain detaches from the emitter) measured on the lattice. On the other hand it is favorable since the bifurcation structure becomes much more visible than in the usual way of plotting bifurcation diagrams (cf. Fig.6.8b). 


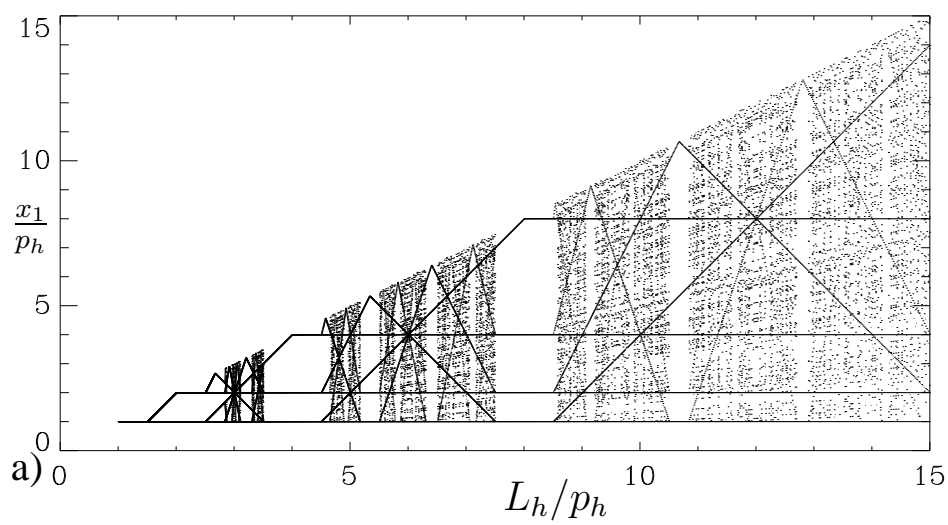

b)
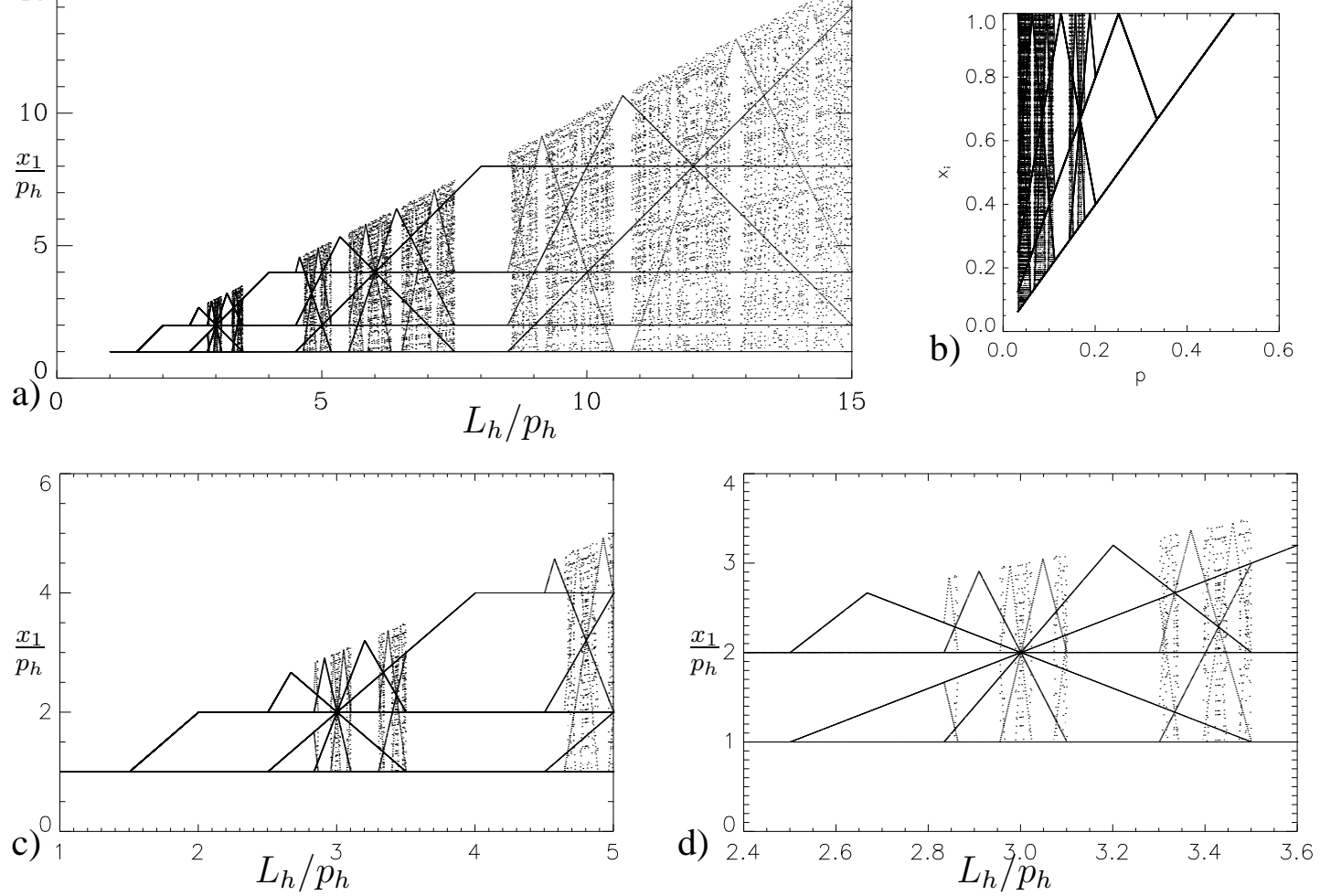

Figure 6.8: Bifurcation diagram of the flat bottom tent map Eq.(6.24) (a). This diagram directly gives the initial domain content on the lattice. b) the bifurcation diagram from a) now plotted in the usual manner with $x=x_{1} / L_{h}$ and $p=p_{h} / L_{h}$, i.e. the bifurcation diagram of the flat bottom tent map as a map of the unit interval onto itself. c),d) enlarged sections of a) for the regions between the period-1 and the period -3 window and between the period- 2 and period- 3 window.

The bifurcation structure of map (6.24) with respect to the bifurcation parameter $p_{h}$ is unusual (Fig.6.8) but has similarities to the flat-topped tent map [53, 101, 95]. Due to the horizontal segments the map cannot show chaotic behavior although it undergoes several bifurcation cascades. A trajectory on the tent map segments would explore the attractor of the tent map and will therefore either fall on one of the flat segments or stay at an unstable periodic orbit of the tent map, which is contained in the remaining part of the tent map. Once arrived at one of the flat segments the trajectory continues on a stable periodic orbit. The dynamics is periodic. By analyzing the $n$ 'th return map of the flat bottom tent map we find, that windows of period $k$ are found inside the intervals $l_{h} / p_{h} \in\left(\frac{2^{k}-1}{4 j-2}, \frac{2^{k}+1}{4 j-2}\right)$ for $j \in I_{k} \subset\left\{j \in \mathbb{N} \mid j \leq, 2^{k-2}-1\right\}$. Thus higher and higher stable periodic orbits are arising in smaller and smaller intervals.

The most obvious feature in Fig.6.8 are the horizontal lines just giving the first, and higher iterated images of the minimal switching height $\left(p_{h}\right)$. Between the windows where with increasing $L_{h} / p_{h}$ orbits of period $k$ and $k+1$ are stable, a point of the 
unstable periodic set of the tent map is reached, which produces the spider like structures with orbits of even periods at one side and odd periods at the other side.

\subsubsection{Obtaining Front Positions}

Now we turn to the front dynamics, driven by the normalized model. The front velocities are given by rule 6.7. If the filling of a domain starts $\left(t=t_{0}\right)$, we set the position of the leading depletion front $d_{i}\left(t_{0}\right)=0$ and the position of the accumulation front that closes the previously filled domain $a_{i}\left(t_{0}\right)=0$.

The evolution of fronts is completely governed by the dynamics of the tank model with respect to the following rule:

Evolve the position of the depletion fronts with velocity $\dot{d}_{i}$ for $i=1, \ldots, N_{d}$ given in rule 6.7 and obtain $a_{i}=d_{i}-L^{h} x_{i}$ for $i=2, \ldots, N_{a}$. If rule 6.18 applies, re-index the front positions in the same manner as $x_{i}$ but do not consider any front position assigned to $x_{i} \mid i<1$. If rule 6.19 applies, re-index the fronts as $x_{i}$ and set $d_{1}=0$.

To compare the results obtained from the hybrid model with the results of a microscopic simulation by means of a sequential tunneling model presented by Amann et al. in [3] we shall consider a transformation to front positions given in units of (quantum) wells. We assume a length of the superlattice of 100 wells as it was used in [3]. To obtain front positions in the superlattice from the normalized model the results have to be rescaled. At first a relation between the normalized total content $\sum_{i} x_{i}=L_{h}$ and the content at the lattice (measured in wells) with respect to voltage $U$ has to be established. We assume that this relation is simply linear and given by:

$$
d_{i}-a_{i-1}=c_{1} U x_{i}
$$

where $c_{1}$ is the scaling constant (in the following we apply: $c_{1}=23[$ wells $/ V]$ ). Further we re-scale the time using the relation between the front velocity $\left(\tilde{v_{d}}\right.$ in wells $\left./ n s\right)$, given in Fig.6.3 and the normalized models front velocity $v_{d}$ by

$$
\tilde{t}=c_{1} U \frac{v_{d}}{\tilde{v}_{d}} t
$$

An adequate approximation is given by the dipole propagation velocity of $V_{d}=V_{a}=$ 10 wells $/ n s$. This value yields $v_{d} / \tilde{v}_{d}=0.1[n s /$ well $]$. Because the relation Eq.(6.6) holds for all models therewith the scaling is done. Figure 6.9 shows an example of the strange billiard like trajectory of the hybrid model and the resulting evolution of domain contents as well as the time evolution of the associated fronts in the superlattice. 


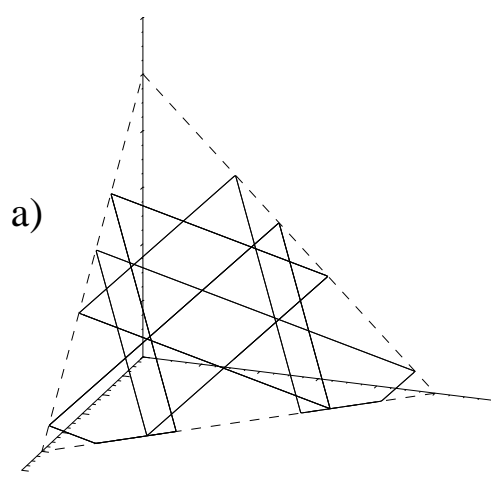

b)

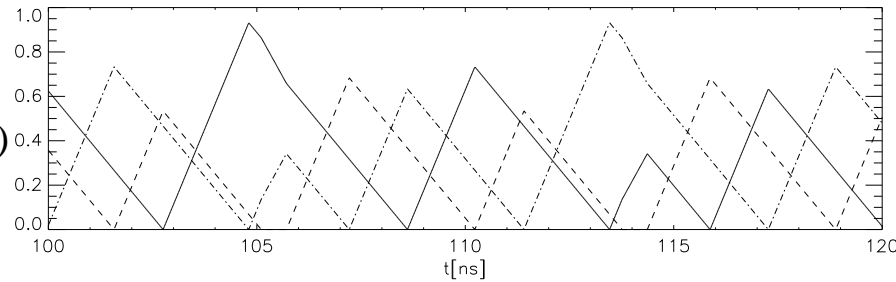

c)

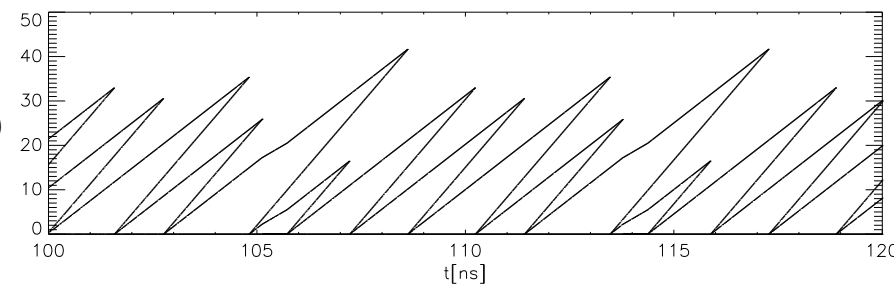

Figure 6.9: Illustration of the basic model including a minimal switching threshold for three domains for $U=0.7 \mathrm{~V}, c_{m}$ in $=5.5$ wells a) The trajectory of domain sizes moves uniformly inside the 3-simplex $S_{3}$ embedded in $\mathbb{R}^{3}$ and if a domain is underneath the threshold along the boundary of $S_{3}$.b) Sample trajectory of domain contents, the time is rescaled by Eq.(6.28). c) Associated evolution of fronts on the lattice, completely rescaled.

We shall briefly discuss the relations between Fig.6.8 and Fig.6.15a that gives a bifurcation diagram of annihilation positions of accumulation and depletion fronts obtained from the hybrid model and rescaled using Eq.(6.27). By rule 6.26 the period of the Poincare map is the period of the pattern observed from the front model. Moreover, the bifurcation diagram of the hybrid model Fig.6.8 indicates the size of the first domain at the moment it is resolved from the emitter. The time $t_{m}$ when this happens is defined by $t_{m}$ of the tank model. Thus the bifurcation diagram Fig.6.8 reveals the structure of Fig.6.15. But the collision position of fronts, although given in principle by $x_{1}\left(t_{m}\right)$, can not be obtained directly in Fig.6.8. During the shrinking of a high-field domain any other waiting tank event may occur, which changes the propagation velocities and therefore the collision position.

\subsubsection{Four and More Domains}

After the above excursion we shall maintain the discussion of the dynamics of the switched arrival tank model given by the rules 6.17-6.19.

For four and more possible domains we obtain a Poincaré map that is hybrid too, due to the likewise possibility that more than one tank stays empty for a wile. For $n=4$ we have only 2 possible discrete states: either none or one tank stays empty $(q=1)$ or two tanks have to wait for filling $(q=2)$. The hybrid Poincaré map can be written as an iterated map of one discrete variable $q$ and two continuous variables $x_{1}, x_{S}$ chosen 
as in Eq.(6.23). For $x_{1}$ in state $q=1$ we can write:

$$
x_{1}\left(t_{m+1}\right)=\max \left\{\min \left\{3 x_{1}\left(t_{m}\right), 3 x_{S}\left(t_{m}\right)\right\}, p_{h}\right\}
$$

The iteration of $x_{S}$ in state $q=1$ depends on the value, obtained for $x_{1}\left(t_{m+1}\right)$. If $x_{1}\left(t_{m+1}\right)>p_{h}$ we have:

$$
x_{S}\left(t_{m+1}\right)=\left\{\begin{array}{lll}
x_{S}\left(t_{m}\right)-x_{1}\left(t_{m}\right) & \text { if } & x_{S}\left(t_{m}\right)>x_{1}\left(t_{m}\right) \\
\min \left\{x_{1}\left(t_{m}\right)-x_{S}\left(t_{m}\right), L_{h}-x_{1}\left(t_{m}\right)-2 x_{S}\left(t_{m}\right)\right\} & \text { if } \quad & x_{S}\left(t_{m}\right)<x_{1}\left(t_{m}\right)
\end{array}\right.
$$

For $x_{1}\left(t_{m+1}\right)=p_{h}$ the additional draining during the time that is needed by the first tank to reach the minimum switching threshold must be considered:

$$
x_{S}\left(t_{m+1}\right)=\left\{\begin{array}{lll}
x_{S}\left(t_{m}\right)-x_{1}\left(t_{m}\right)-\frac{p_{h}-x_{1}}{2} & \text { if } & x_{S}\left(t_{m}\right)>x_{1}\left(t_{m}\right) \\
\min \left\{x_{1}\left(t_{m}\right)-x_{S}\left(t_{m}\right)-\frac{p_{h}-x_{s}}{2},\right. & & \\
\left.L_{h}-x_{1}\left(t_{m}\right)-2 x_{S}\left(t_{m}\right)-\frac{p_{h}-x_{s}}{2}\right\} & \text { if } & x_{S}\left(t_{m}\right)<x_{1}\left(t_{m}\right)
\end{array}\right.
$$

In state $q=1$ the following discrete state is obtained by:

$$
q\left(t_{m}+1\right)=q\left(t_{m}\right) \text { if } X_{S}\left(t_{m+1}\right)>0 \text { and } q\left(t_{m}+1\right)=2 \text { otherwise }
$$

If $q\left(t_{m}+1\right)=2$ we have to reset (see Sec.2.2.1) the continuous state by replacing $x_{S}$ by:

$$
x_{S}\left(t_{m}+1\right)=1-p_{h}
$$

Once arrived in the discrete state $q=2$ (i.e. two waiting tanks during $\left(t_{m}, t_{m+1}\right)$ the iteration of the hybrid Poincaré map is straightforward:

$$
\begin{aligned}
& x_{1}\left(t_{m+1}\right)=p_{h} \\
& x_{S}\left(t_{m+1}\right)=\min \left\{x_{S}\left(t_{m}\right)-\frac{p_{h}}{2}, \frac{p_{h}}{2}\right\} \\
& q\left(t_{m+1}\right)=1
\end{aligned}
$$

Basically this map is most time a two dimensional iterated map (state $q=1$ ) and only sometimes it becomes for some iterations one dimensional $(q=1)$. Since we have to proceed with a two dimensional map after every $q=2$ state we keep the second variable $x_{s}$ even there.

The bifurcation structure of this hybrid map is shown in Fig.6.10. Although the bifurcation structure is much more complicated than for the $n=3$ case the same basic features are obtained: Here we have again the horizontal lines of images of the bifurcation parameter $p_{h}$, and even the spider like structures appear.

For more than four possible domains, in other word for $r_{c} \geq 3 / 4$, the hybrid Poincaré map has a higher dimension and $n-2$ discrete states. The numerics indicates, that even in this case horizontal lines and spider structures are characteristics of the bifurcation 

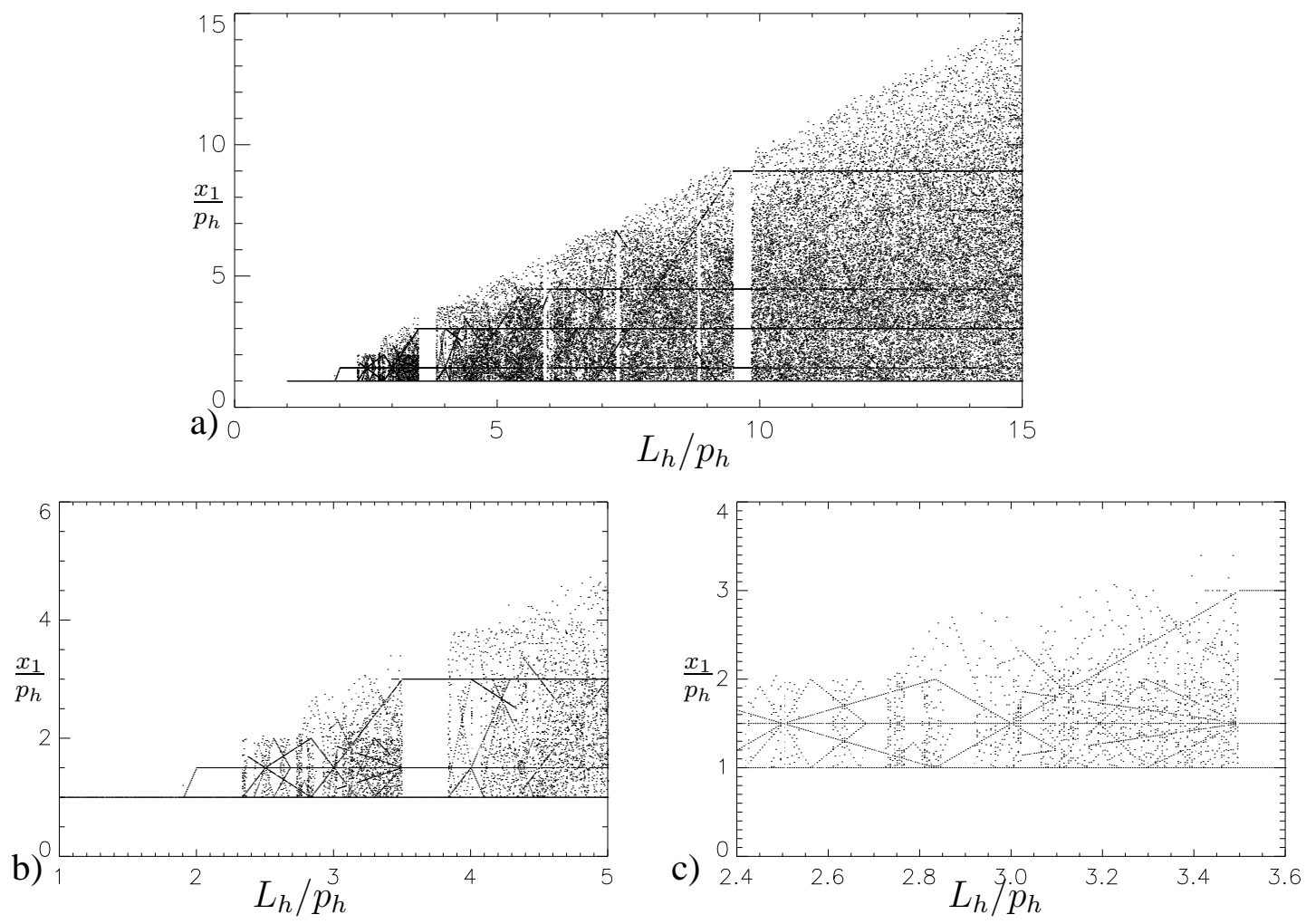

Figure 6.10: Bifurcation diagram of the hybrid map Eq.(6.29)-Eq.(6.34). a) This diagram directly gives the initial domain content on the lattice. b),c) enlarged sections of a).

diagrams. However, we think that it is beyond the scope of this thesis to investigate the bifurcations of more dimensional piecewise linear hybrid maps in detail. Since here not only an example of such a map, but also an application in a highly attracting area of modern semiconductor physics is outlined, we hope to stimulate future research on such bifurcation problems.

\subsection{Dynamics if Domains Can Traverse the Sample}

A second effect that has to be included in the hybrid model is the run out of fronts reaching the collector due to the limited size of the lattice. To decide whether a front has reached the collector or not, we have to include an additional set of variables $\left\{d_{i} \mid i=1, \ldots, N\right\}$ into the model, because $d_{i}$ is not a unique function of $x$ (it depends on the history). Now we deal with a hybrid model that contains exactly as many continuous variables as fronts are present in the superlattice.

If $d_{i}\left(t_{0}\right)=L$ we assume that at first no new dipole is injected at the emitter site. Thus we get, due to $N_{a}=N_{d}$ new front velocities: $v_{d}^{*}=v_{a}^{*}=1$ and therefore the domain, that is at the collector site will be emptied with $\mu^{*}=1$ while for all other domains 


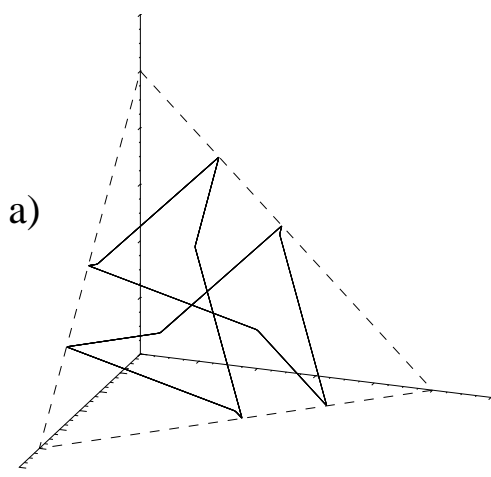

b)

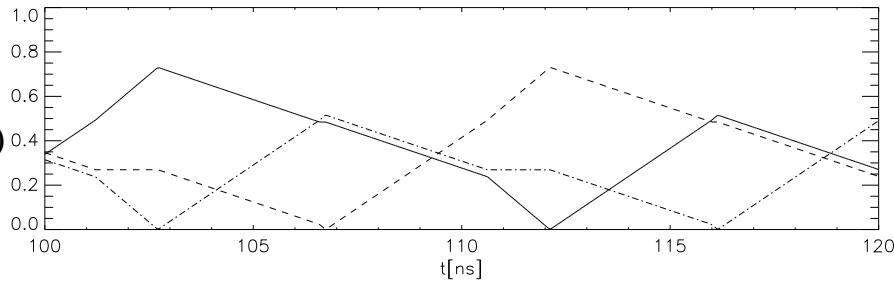

c)

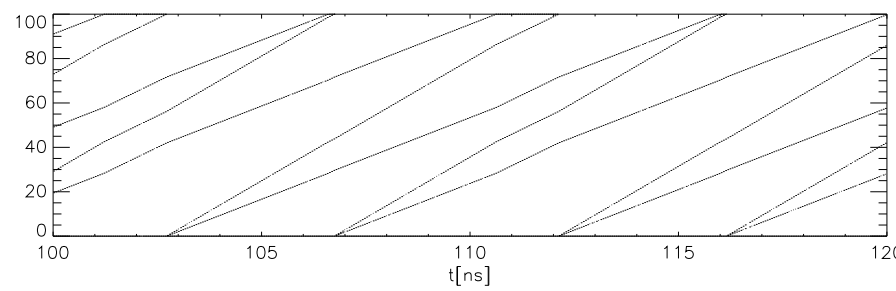

Figure 6.11: Illustration of the model including with a periodic solution for all fronts reaching the collector at $U=2.5 \mathrm{~V}$. a) The trajectory of domain sizes moving inside the 3-simplex $S_{3}$ embedded in $\mathbb{R}^{3}$. b) Sample trajectory of domain contents. c) Associated evolution of fronts on the lattice.

$\mu^{* *}=0$ and the one attached to the emitter will be filled with $\lambda^{*}=\mu^{*}$. With these considerations we have to add one additional discrete state to the model. Finally, if the domain at the collector is empty, a new dipole is injected, and the system turns back to its normal state. During the time the domain at the collector is emptied, no other domain can become empty. Thus the rules prevent a conflicting situation. Although a unreal situation is not avoided: if a new domain at the emitter is opened just before another reaches the collector, we may get an accumulation and depletion front running through the lattice in a small distance.

More formally we represent the mechanism outlined above by including rule 6.12 beside the rules 6.17-6.19 and 6.26 in a complete hybrid model for the front dynamics. With this model various dynamical regimes are numerically obtained. This includes periodic states, where all domains reach the collector (see Fig. 6.11) as well as higly complex patterns (Fig.6.12).
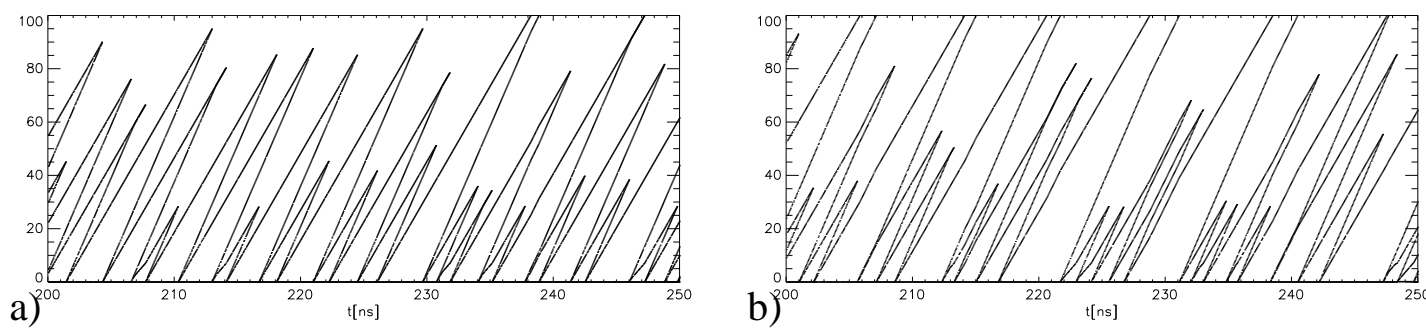

Figure 6.12: By means of the hybrid model numerically obtained patterns of front dynamics with $n=4$ and fronts reaching the collector. A length of the lattice of 100 wells is assumed and the results are rescaled according to Eq.(6.27) and Eq.(6.28) a) $L_{h} / p_{h}=16$ b) $L_{h} / p_{h}=20$ 


\subsection{Comparison of Numerical Results}

To test the validity of the hybrid model for front dynamics in semiconductor superlattices we will now compare some numerical results obtained from the much simpler hybrid model with results of the full microscopic sequential tunneling model given in [3]. At first we consider the spatio-temporal patterns of moving accumulation and depletion front (see Fig.6.5 and Fig.6.14). It turns out, that the results in [3] are obtained for the $n=3$ case (see Sec.6.3.3).

a)

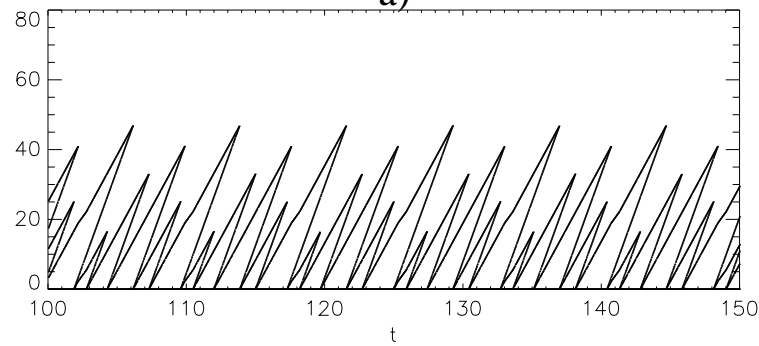

b)

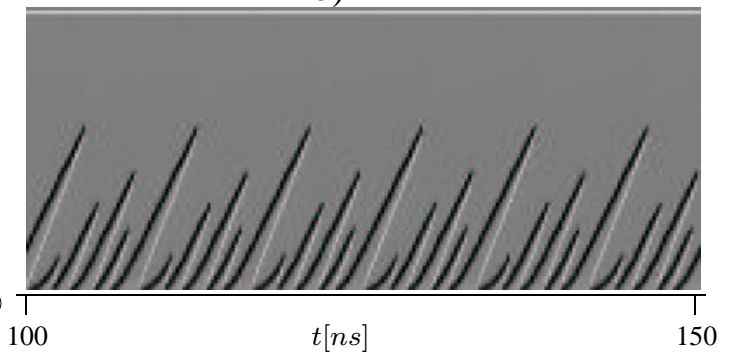

Figure 6.13: Dynamic evolution of front positions , obtained in a period-5 window. from the hybrid model (a) and the microscopic sequential tunneling model(b) [3]. The position is given in wells for a superlattice of 100 wells. a): $n=3, L_{h} / p_{h}=3.2$, rescaled according to Eq.6.27 and Eq.6.28. b) $\sigma=0.5 \Omega^{-1} \mathrm{~m}^{-1}, U=0.95 \mathrm{~V}$.

Generally the patterns for small voltages are reproduced qualitatively satisfactionable with the simplified three tank model including a minimal switching height. Especially the time intervals between successive maxima in the pattern are in good coincidence if the scaling is well adapted (cf. Fig. 6.14). The annihilation positions are not completely reproduced, but a discretisation phenomenon in the sequential tunneling model should be considered, leading to an earlier annihilation if the difference between fronts is smaller than one well. It seems that a drift of the parameter ${ }_{h}$ from higher to lower values with increasing voltage can give better coincidences. Basically the domains obtained from the hybrid model seem to be too large in an arbitrary emitter distance. This coincides with the observation that the dynamics near the emitter site is not well reproduced in the hybrid model. However, we do not expect to reproduce the dynamics of the microscopic model in all details by the hybrid model. Especially the complicated interactions during the detachment of fronts are not considered in the hybrid modeling. But the hybrid model, based on very basic assumptions is, as the examples show able to capture the essential features of the spatio-temporal pattern formation process.

The bifurcation diagram 6.15 shows, that the bifurcation structure for small voltages can be understood from the hybrid model. The simple linear scaling relation which we use here (Eq.(6.27)) is not proper (especially the width of the periodic win- 

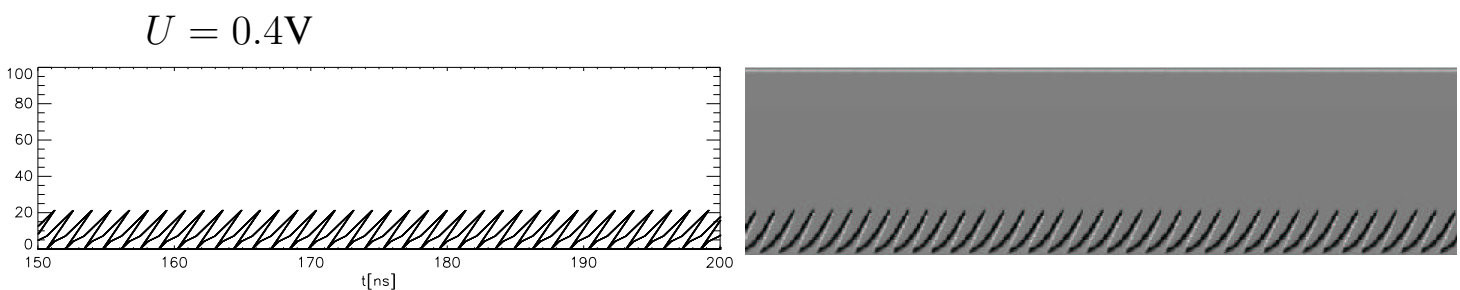

$U=0.7 \mathrm{~V}$
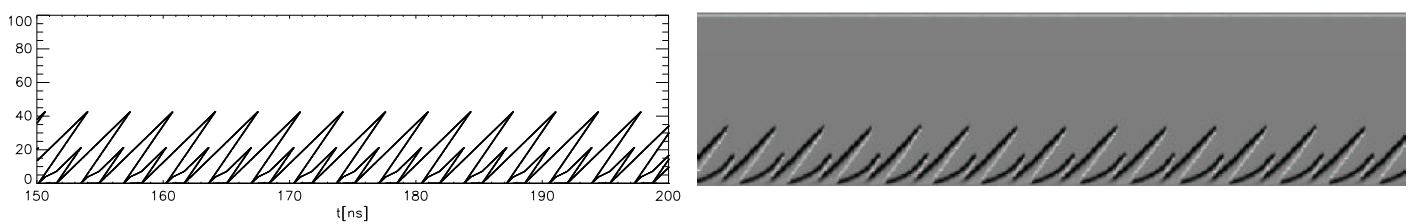

$U=0.82 \mathrm{~V}$
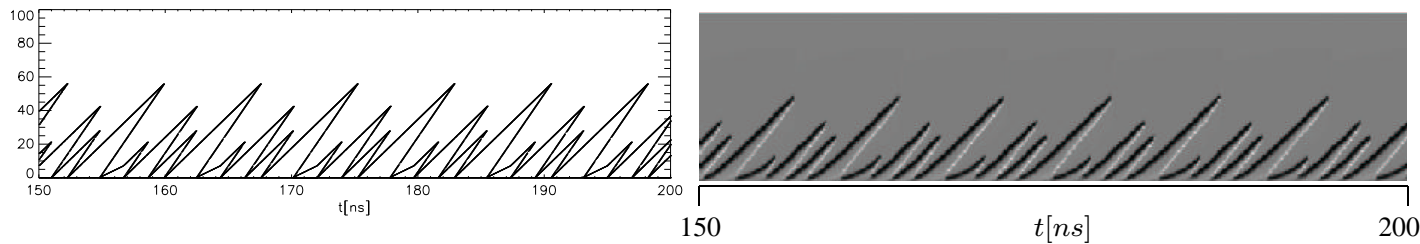

Figure 6.14: Dynamic evolution of front positions for various voltages, obtained from the hybrid model (first column) and the sequential tunneling model [3] at $\sigma=0.5 \Omega^{-1} \mathrm{~m}^{-1}$. The voltages applied to the superlattice are indicated.

dows is too large), and some details, visible in Fig.6.15b are not present in Fig.6.15a. I.e. the falling property of some branches after bifurcations, and the clearly reduced minimal annihilation position inside the second "chaotic" window above $U=1.1 \mathrm{~V}$. A possible explanation is, that the microscopic simulations are carried out with parameters, that give $r_{c} \approx 2 / 3$. The microscopic simulations therefore are near the situation where we have to operate with a $n=4$ tank model [4]. Thus in the regions above the prominent period-3 window sometimes the system simulated with the full microscopic model falls in irregular dynamical regimes by opening a fourth domain that annihilates near the emitter.

We note, that the prominent features of the bifurcation diagram, for instance the horizontal lines, given by the first, and higher iterated images of the minimal filling height are covered completely by the observations of the one-dimensional flat bottom tent map. In particular the spider-like structure at $U \approx 0.9 \mathrm{~V}$ in Fig.6.15 is explained by the nature of the bifurcation structure of the flat bottom tent map Eq.(6.24) (cf. Fig.6.8). 

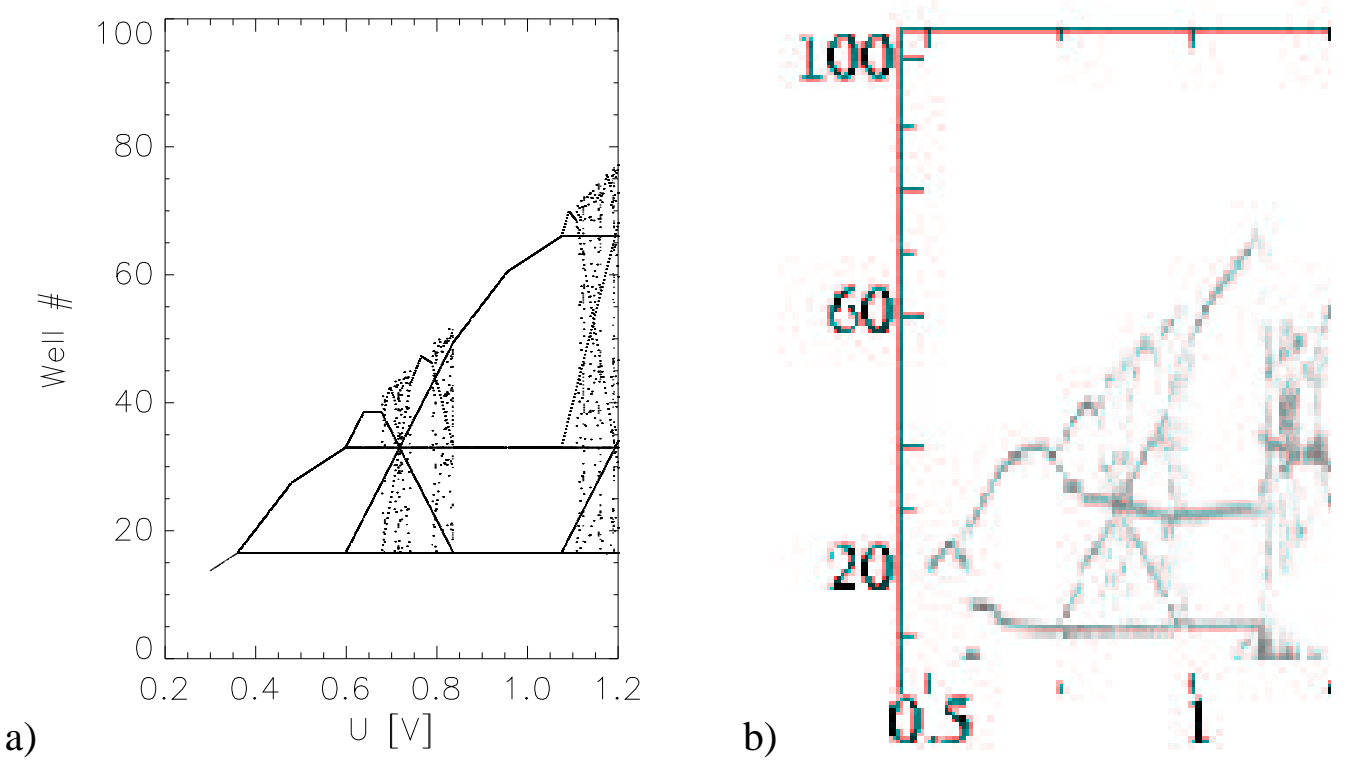

Figure 6.15: Bifurcation diagrams of positions where accumulation and depletion fronts annihilate, obtained (a) from the hybrid model with three tanks (see Sec. 6.3.3) and (b) a simulation by means of a sequential tunneling model [3].

\subsection{Conclusion}

We have introduced hybrid models as simplified models for the front dynamics in semiconductor superlattices. These hybrid models are derived by idealizing the continuous but very fast processes of front generation and annihilation as instantaneous switching processes. The global coupling in the superlattice system in connection with the integral over space constraint allowed a hybrid modeling of the semiconductor system by means of a switched arrival system with $n$ tanks. In this unexpected analogy, the tank filling level corresponds to the widths of the high-field domains in the superlattice, while the position of the server determines which high field domain is connected to the emitter. The number of tanks that have to be included are directly given by a physically meaningful constant $r_{c}$. The switched arrival system includes a minimal switching threshold $p_{h}$ that was motivated by the process of front detachment in the superlattice. This minimal switching threshold leads to a special bifurcation structure that was investigated in particular for $n=3$. For this case a one dimensional map named flat bottom tent map is found, which covers the front dynamics as long as no front reaches the collector completely. This simplified model provides an explanation for the complicated front dynamics in semiconductor superlattices. A bifurcation diagram for the positions of the front collisions (Fig. 6.15) exhibits a striking 
similarity with the corresponding diagram of the full sequential tunneling model [3]. Although we investigated a superlatticemodel in this chapter, the presented methods are very general and are expected to work for other pattern forming systems which provide bistability and a global coupling similar to Eq.(6.3) as well. 


\title{
7 Conclusions and Perspectives
}

\author{
Here the results and insights obtained in the previous chapters are summarized. Some \\ open questions concerning the dynamics of hybrid systems and related topics are \\ outlined and conclusions for future research are drawn.
}

In this thesis new results for the dynamics of hybrid switched tank systems have been presented. By using hybrid switched tank systems as models for manufacturing systems and front dynamics in semiconductor superlattices new insights are obtained into the nature of the dynamics of these systems.

The switched tank systems considered throughout this thesis deserve interest in their own right. On the one hand side they are fundamental models for the dynamics of hybrid systems, because all complexities of the dynamics are produced by the hybrid characteristics of the systems, i.e. the rule dependent switching processes. Furthermore, they are ideal model systems to study border collision bifurcations because these are the only bifurcations that can occur due to the piecewise linear nature of the associated Poincaré maps. On the other hand, the large number of different border collision bifurcations (see Chap.3) causes the rich variety of dynamical behaviors in switched tank systems.

By considering switched server systems and switched arrival systems with upper switching thresholds we found a close relation between these models. We have seen, that a switched server system in a limiting case can show the behavior of a switched arrival system and vice versa (Sec.4.2).

In particular we investigated the dynamics of some close relatives and variants of a three tank switched server system in detail. In Sec.4.3 an upper threshold for all tanks was applied and chosen as bifurcation parameter. The bifurcations of these systems, as of all other systems considered here as well, are exclusively border collision bifurcations. We have discussed the bifurcation mechanisms. We remark that border collision bifurcations in these systems are indeed associated with collisions of the attractor with a simplex boundary, when changing a control parameter. In Sec.4.4.1 we discussed the influence of various switching rules on the dynamics of these systems. A three tank system with an upper threshold applied only to the first tank was considered in Sec.4.4.2. Here the value of one filling rate $\left(\lambda_{2}\right)$ was identified to be crucial for the system's ability to exhibit chaotic behavior. A system that contains two 
servers, one for filling two tanks and another for emptying two tanks, was investigated in Sec.4.4.3. For this system a hybrid Poincaré map was obtained. This is an iterated map, in which the iteration of a continuous variable depends on a discrete state. In the context of manufacturing systems a switched tank model with additional set-up times was introduced (Sec.5.2). Here the Poincare map is also hybrid. Although the system's description contains a higher number of discrete states, the dynamics of these systems, including a set-up time was found to be similar to the dynamics of systems without set-up times.

Whereas in Chap. 4 and Chap. 5 all possible choices of rates were of interest, in Chap.6, where a model for the front dynamics was investigated, all tanks obtained equal filling rates. By using the symmetries that are introduced therewith, modified versions of the tent map could be used for the description of the dynamics. Due to the introduction of minimal switching thresholds, motivated by physical reality, the associated three tank switched server system shows only periodic dynamics. The period, however, can be very high.

From the investigations of a variety of hybrid switched tank systems we conclude, that seemingly slight changes in the model can have major consequences for the dynamical features. Furthermore, the inclusion of conditions, like set-up times, leads in a natural way to hybrid Poincaré maps.

Since the beginnings of nonlinear dynamics the theoretical concepts were applied to problems of interdisciplinary character in all areas of science, including physics, ecology, economics and biology. In this thesis we applied the knowledge, obtained theoretically for a general description of switched arrival systems and switched server systems to investigate systems exhibiting a complex dynamics from two areas of science.

We addressed the dynamics of basic configurations in automated manufacturing systems. Here we have approximated the discrete flow of materials by a continuous process to obtain a hybrid model that describes the dynamics and can be analyzed by analytical means from nonlinear dynamics. The irregularities obtained in such systems are shown to be caused by the intrinsic dynamics of policy guided switching systems. Restricted buffer capacities in switched server systems are identified as a source of chaotic behavior in the hybrid models of switched server configurations. By means of numerical simulation we have seen, that the chaotic behavior and bifurcations, obtained for a continuous approximation of material flows are qualitatively also found for discrete deterministic queuing models. To clarify the interdependencies between systems parameters, dynamics and performance we used measures that are used regularly in operations research and by production engineers. It became obvious, that chaotic behavior not only complicates the planning of production but also reduces effectively the productivity. The question, what countermeasures are able to prevent such drawbacks was briefly discussed. The knowledge and methods for predicting the 
effects of changed parameters or policies are contained in this thesis.

The most unexpected result of this thesis is the application of a switched arrival tank model to describe the dynamics of accumulation and depletion fronts in semiconductor superlattices. By approximating basically continuous processes of front generation and annihilation by instantaneous switching-like processes, it was possible to find a generic description for this spatio-temporal pattern formation process. The global coupling in the superlattice in connection with an integral over space constraint allowed a model, in which the tank filling levels correspond to the widths of high-field domains in the superlattice, while the position of the server determines, which high-field domain is connected to the emitter. With this model the complex pattern formation process and a bifurcation scenario, obtained by means of a simulation of a sequential tunneling model, could be explained and investigated by means of a one dimensional map, called flat bottom tent map. Although we investigated a superlattice model, the applied methods are very general and are expected to work for other pattern forming systems, too. This might be useful in other areas of research, where systems with similar characteristics, i.e. a global coupling and a bistability inducing fronts with different characteristic velocities appear.

From the presented results it becomes obvious, that a hybrid modeling approach can be a highly efficient method to obtain idealized models of dynamical phenomena, describing the main features of the dynamics. Furthermore, at least the models considered here are tractable by analytical means. This is possible due to the piecewise linear nature of the obtained Poincaré maps. For some systems we could make use of reduced return maps summarizing the action of whole paths in a state transition diagram of the hybrid system in one segment of the map to simplify the analysis. The hybrid nature of the systems is reflected by the existence of not only the above mentioned Poincaré map, but also a second map that has to describe the dynamics of inter-switching times of the systems (Sec.4.3.3) and state transition graphs that are a way to determine the possible evolution of discrete states in these hybrid systems.

As we have seen, the investigation of more complicated hybrid systems leads in a natural way to hybrid maps. Since those maps are the most simple hybrid systems a future investigation of hybrid dynamical phenomena may consider such hybrid maps as starting point for a systematic research.

In connection with the dynamics of hybrid systems a number of problems is still unresolved. This ranges from very fundamental questions, as for instance the classification and investigation of border collision bifurcations in more than two dimensions, to a new view on natural systems which obey characteristics as the pattern formation process in superlattices that can be covered by a hybrid modeling approach.

We expect that if the concept of hybrid systems becomes more known among researchers from natural sciences even other systems will be recognized where this notion may lead to new insights. Especially networks of pulse coupled oscillators (integrate-and fire neuron models) [44, 96] are seemingly hybrid. 
A closer investigation of hybrid systems with impulse like transitions of the continuous state may be fruitful. It will be a challenge to reach furthermore a deeper understanding of the implications of mixed occurrence of state and timed events in the same system as we have seen them in the switched server model with set-up times. Up to now we lack a quantitative measure for the chaotic properties hybrid systems. For strange billiard like systems as considered here it may be promising to explore a transfer of methods used for the estimation of Lyapunov exponents in Hamiltonian billiards $[37,36,38]$ to strange billiards.

In this thesis not only models are presented, that reveal some fascinating features, but also an application in a highly attracting area of modern physics was given. We hope that this will stimulate future research in the field of hybrid dynamical systems. 
A Tables of Poincaré Maps 


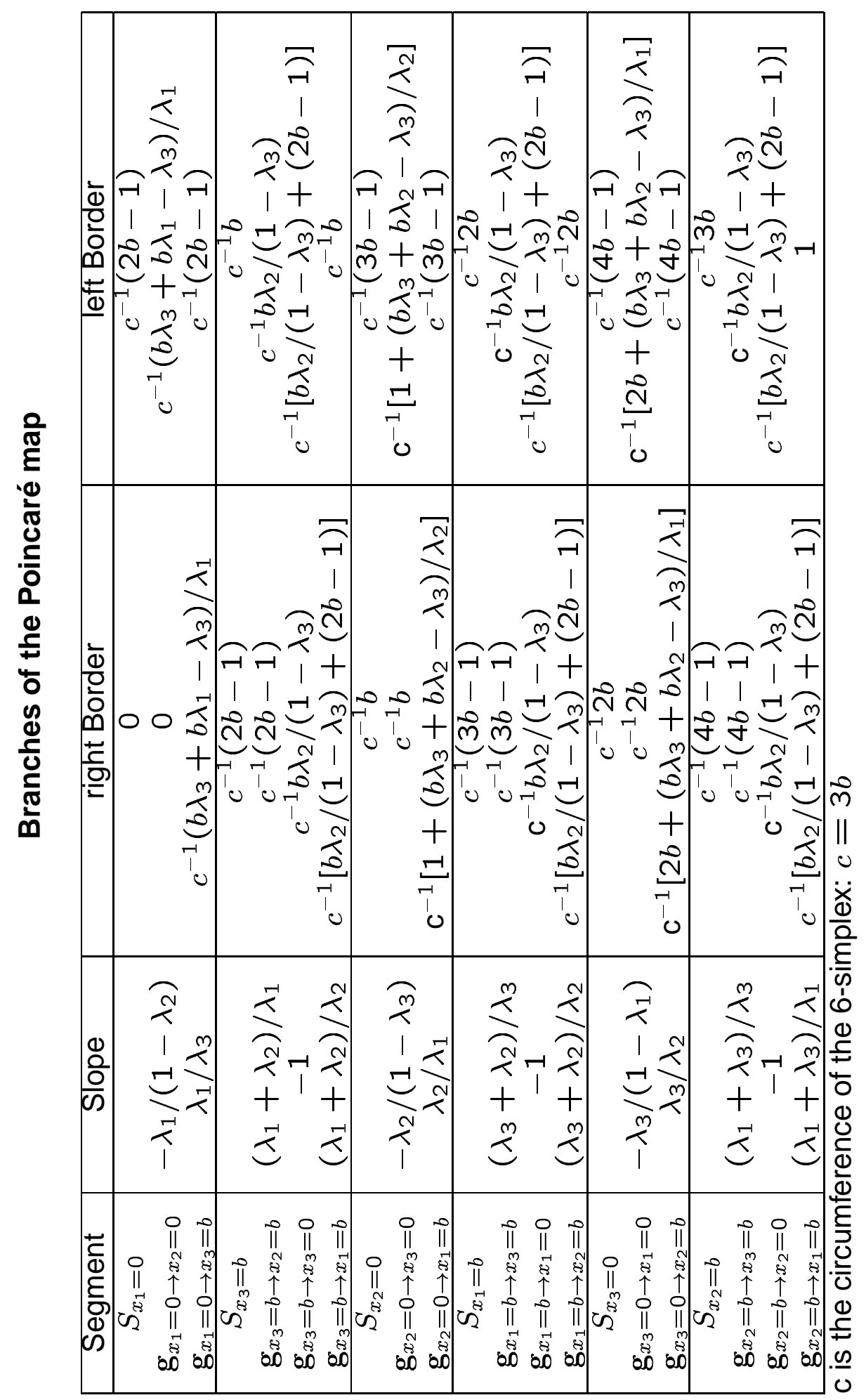

Table A.1: Table of the segments of the Poincare map for the three tank switched server system with a maximum tank capacity $b$ for all tanks. 


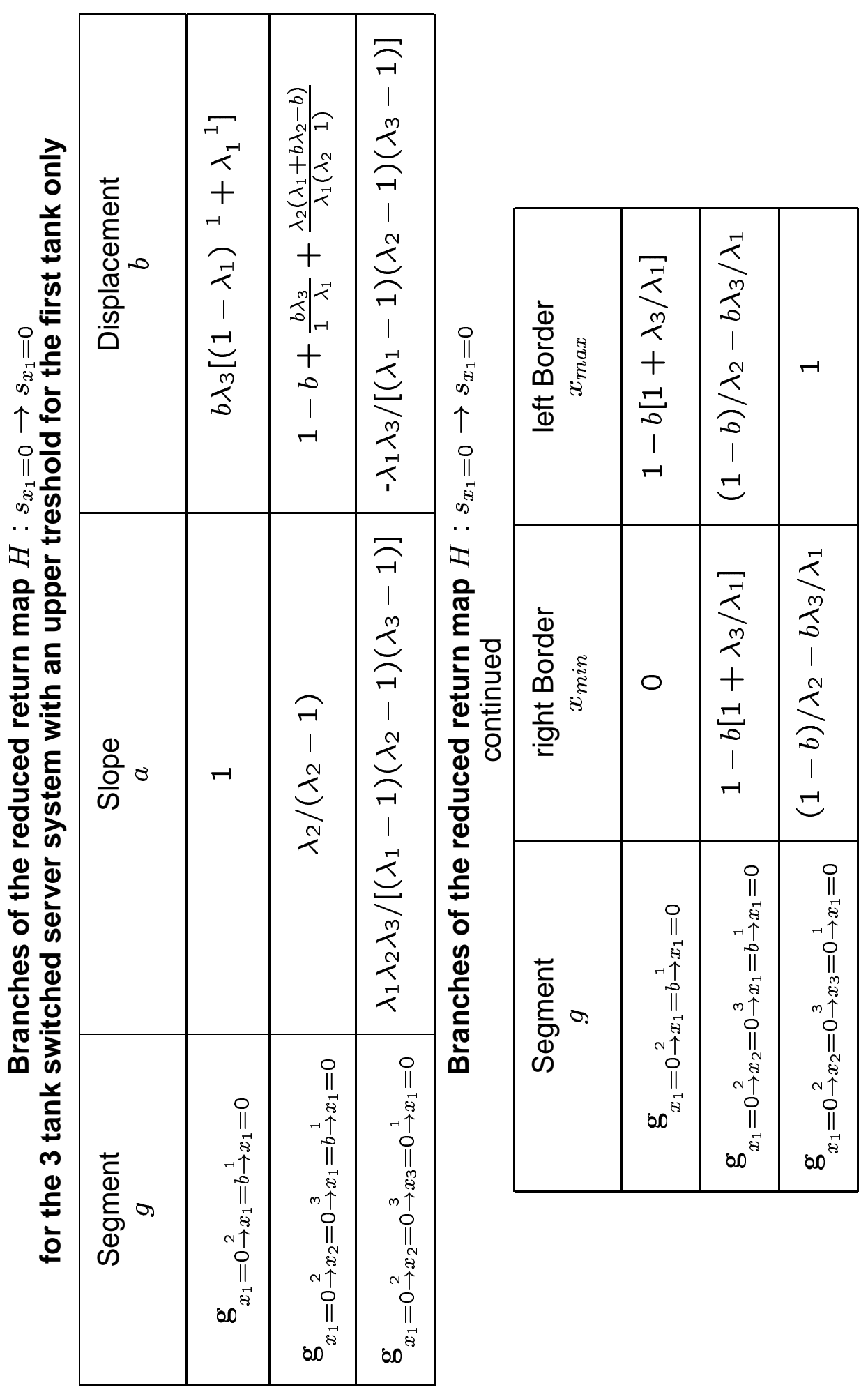

Table A.2: Table of the segments of the reduced Poincaré map for the three tank switched server system with an upper threshold $b$ assingned to the first tank only. 



\section{Bibliography}

[1] R. Alur, C. Belta, F. Ivancic, V. Kumar, M. Mintz, G.J. Pappas, H. Rubin, and J. Schung. Hybrid Modeling and Simulation of biomolecular Networks, pages 19-32. Number 2034 in LNCS. Springer-Verlag, Berlin, Heidelberg, New York, 2001.

[2] R. Alur, C. Belta, V. Kumar, M. Mintz, G.J. Pappas, H. Rubin, and J. Schug. Modeling and analyzing biomolecular networks. Computing in Science Engineering, 4(1):2-13, 2002.

[3] A. Amann, J. Schlesner, A. Wacker, and E. Schöll. Chaotic front dynamics in semiconductor superlattices. Phys. Rev. B, 65:066207, 2002.

[4] A. Amann and J.H. Schlesner. private communication.

[5] A. Amann, A. Wacker, L.L. Bonilla, and E. Schöll. Dynamic scenarios of multistable switching in semiconductor superlattices. Phys. Rev. E, 63:193313, 2002.

[6] V. Avrutin and Schanz M. On the scaling properties of the period increment scenario in dynamical systems. Chaos, Solitons and Fractals, 11:1949-1955, 2000.

[7] S. Banerjee and C. Grebogi. Border collision bifurcations in two dimensional piecewise smooth maps. Phys. Rev. E, 59(4):4052-4061, 1999. see Erratum PRE60(3)3450.

[8] S. Banerjee, M.S. Karthik, G. Yuan, and J.A. Yorke. Bifurcations in onedimensional piecewise smooth maps-theory and applications in switching circuits. IEEE Trans. Circuits and Syst.-I: Fund. Theory and Appl., 47(3):389394, 2000.

[9] S. Banerjee, P. Ranjan, and C. Grebogi. Bifurcation in two-dimensional piecewise smooth maps - theory and applications in switching circuits. IEEE Trans. Ciruits Syst. I: Fundam. Theory Appl., 47:633-643, 2000.

[10] S. Banerjee, J.A. Yorke, and C. Grebogi. Robust chaos. PRL, 80(14):30493052, 1998.

[11] J.J. Bartholdi, L.A. Bunimovich, and D.D. Eisenstein. Dynamics of twoand three worker "bucket brigade" production lines. Operations Research, 47(3):488-491, 1999. 
[12] J.J. Bartholdi and D.D. Eisenstein. A production line that balances itself. $O p$ erations Research, 44(1):21-34, 1996.

[13] T. Beaumariage and K. Kempf. The nature and origin of chaos in manufacturing systems. In 5th IEEE/SEMI Adv. Semicond. Manufacturing Conf., pages 169174, 1994.

[14] C. et al. Belta. Satbility and reachability analysis of a hybrid model of luminescence in the marine bacterium v.fischri. Grasp. Lab., Univ. Pennsylvania, Philadelphia, 2002.

[15] A. Bemporad, G. Ferrari-Trecate, and M. Morari. Observability and controllability of piecewise affine and hybrid systems. IEEE Trans. Autom. Control, 45(10):1864-1876, 2000.

[16] L. Billings and E.M. Bollt. Probability density functions of some skew tent maps. Chaos, Solitons and Fractals, 12:365-376, 2001.

[17] M. Branicky. Studies in Hybrid Systems: Modeling, Analysis and Control. $\mathrm{PhD}$ thesis, Massachusetts Institute of Technology, 1995.

[18] M. Branicky. General hybrid dynamical systems, volume 1066 of LNCS, pages 186-200. Springer Verl., 1996.

[19] M.S. Branicky. Multiple lyapunov functions and other analysis tools for switched and hybrid systems. IEEE Trans. Autom. Control, 43(4):475-482, 1998.

[20] M.S. Branicky, V.S. Borkar, and S.K. Mitter. A unified framework for hybrid control: Backround, model and theory. In Proc. 33rd IEEE Conference on Decision and Control, Lake Buena Vista, 1994.

[21] O.M. Bulashenko and L.L. Bonilla. Chaos in resonant-tunneling superlattices. Phys. Rev. B, 52(11):7849-7852, 1995.

[22] L. Bunimovich. Dynamical systems and operations research: a basic model. Discrete and Continuous Dynamical Systems B, 1(2):209-218, 2001.

[23] I.R. Cantalapiedra, M.J. Bergmann, L.L. Bonilla, and S.W. Teitsworth. Chaotic motion of space charge wave fronts in semiconductors under time independent voltage bias. Phys. Rev. E, 63:056216, 2001.

[24] A. Carpio, L.L. Bonilla, A. Wacker, and E. Schöll. Wave fronts may move upstream in semiconductor superlattices. Phys. Rev.E, 61(5):4866-4876, 2000.

[25] C. Chase, J. Serrano, and P.J. Ramdge. Periodicity and chaos from switched flow systems: Contrasting examples of discretely controlled continuous systems. IEEE Transact. Autom. Contr., 38(1):70-83, 1993.

[26] J. Chen and M.A. Reed. Electronic transport of molecular systems. Chem. Phys., 281:127-145, 2002.

[27] W. Chin, E. Ott, H.E. Nusse, and C. Grebogi. Grazing bifurcations in impact oscillators. Phys. Rev. E, 50(6):4427-4444, 1994. 
[28] B. Christiansen, D.-R. He, S. Habip, M. Bauer, U. Krueger, and W. Martienssen. Phase diagram of a modulated relaxation oscillator with a finite restting time. Phys. Rev. A, 45(12):8450-8456, 1992.

[29] S. Coombes and A.H. Osbaldestin. Period adding bifurcations in a periodically stimulated exitable neural relaxation oscillator. Phys. Rev. E, 62(3):4057-4065, 2000.

[30] J.D. Crawford. Introduction to bifurcation theory. RMP, 63(4):991-1037, 1991.

[31] B. Cristiansen, P. Alstrom, and M.T. Levinsen. Routes to complete phese locking in modulated relaxation oscillators. Phys. Rev. A, 42(4):1891-1900, 1990.

[32] M.C. Cross and P.C. Hohenberg. Pattern formation outside of equilibrium. Rev. Mod. Phys., 65(3):851-1112, 1993.

[33] W. de Melo and S. van Strien. One -dimensional Dynamics. Springer Verl. Berlin, 1993.

[34] J. de Wegner, W. van de Water, and J. Molenar. Grazing impact oscillations. Phys. Rev. E, 62(2):2030-2041, 2000.

[35] R.A. Decarlo, M.S. Branicky, S. Petterson, and B. Lennartson. Perspectives and results on the stability and stabilizity of hybrid systems. Proc. IEEE, 88(7):1069-1082, 2000.

[36] C. Delago, L. Glatz, and H.A. Posch. Lyapunov spectrum of the driven lorentz gas. Phys. Rev. E, 52(5):4817-4825, 1995.

[37] C. Delago and H. Posch. Lyapunov eponents of systems with elastic hard collisions. Phys. Rev. E, 52(3):2401-2406, 1995.

[38] C. Dellago and W.G. Hoover. Are local lyapunov exponents continuous in phase space? Phys. Lett. A, 268:330-334, 2000.

[39] M. di Bernado, C.J. Budd, and A.R. Champneys. Grazing and border-collision in piecewise smooth systems: A unified analytical framework. Phys. Rev. Lett., 86(12):2553-2556, 2001.

[40] M. di Bernardo, M. Feigin, S. Hogan, and M. Homer. Local analysis of cbifurcations and chaos in n-dimensional piecewise smooth systems. Chaos, sSolitons and Fractals, 10(11):1881-1908, 1999.

[41] I. Diaz-Rivera. The dynamics of queues of re-entrant manufacturing systems. $\mathrm{PhD}$ thesis, Arizona State University, 1997.

[42] I. Diaz-Rivera, D. Armbruster, and T. Taylor. Periodic orbits in re-entrant manufacturing systems. to be published, 2002.

[43] M. Dutta, H.E. Nusse, E. Ott, J.A. Yorke, and G. Yuan. Multiple attractor bifurcations: a source of unpredictability in piecewise smooth systems. Pys. Rev. Lett., 83(21):4281-4284, 1999.

[44] U. Ernst, K. Pawelzik, and T. Geisel. Delay induced multistable synchronization of biological oscilators. PRE, 57(2):2150-2162, 1998. 
[45] A. Erramilli and L.J. Forys. Oscillations and chaos in a flow model of a switching system. IEEE Journal on Selected Areas in Communications, 9(2):171-178, 1991.

[46] L. Esaki and L.L. Chang. New transport phenomenon in a semiconductor superlattice. Phys. Rev. Lett., 33(8):495-498, 1974.

[47] L. Esaki and R. Tsu. Superlattice and negative differential conductivity in semiconductors. IBM j. Res. Develop., 14:61, 1970.

[48] J.B. Evans. Structures of discrete event simulation. Ellis Horwood Lim., Chichester, 1988.

[49] M.I. Feigin. Doubling of the oscillation period with c-bifurcations in piecewise continuous systems. PMM [Journ. Appl. Maths. Mech.], 34:861-869 [822830], 1970.

[50] M.I. Feigin. On the generation of sets of subharminic modes in a piecewisecontinuous system. PMM [Jour. Appl. Math. Mech.], 38(5):810-818 [759767], 1974.

[51] M.I. Feigin. On the structure of c-bifurcation boundaries of piecewisecontinuous systems. PMM [Journ. Appl. Math. Mech.], 42(5):820-829 [885895], 1978.

[52] R. Gilmore. Topological analysis of chaotic dynamical systems. Rev. Mod. Phys., 70(4):1456- 1529, 1998.

[53] L. Glass and W. Zeng. Bifurcations in flat-topped maps and the control of cardiac chaos. Int. Journ. Bif. Chaos, 4(4):1061-1067, 1994.

[54] H.T. Grahn, R.J. Haug, W. Müller, and K. Ploog. Electric-field domains in semiconductor superlattices: A novel system for tunneling between $2 \mathrm{~d}$ systems. Phys. Rev. Lett., 67(12):1618-1621, 1991.

[55] D. Hanson, D. Armbruster, and T. Taylor. On the stability of reentrant manufacturing systems. In Beghi et al., editor, Poc. of the 31st MTNS, Padua 1998: Mathematical Theory of Networks and Systems, 1999.

[56] L.H. Hartwell, J.J. Hopfield, S. Leibler, and A.W. Murray. From molecular to modular cell biology. Nature, 402(6761Suppl):C47-52, 1999.

[57] W.P.M.H. Heemels, B. de Schutter, and A. Bemporad. Equivalence of hybrid dynamical models. Automatica, 37(7):1085-1091, 2001.

[58] J.E. Hopfcroft and J.D. Ullman. Introduction to automata theory, languages and computation. Addison Wesley, 1979.

[59] C. Horn and P. J . Ramadge. A. topological analysis of a family of dynamical systems with non-standard chaotich and periodic behaviour. Int. Journ. Control, 67(6):979-996, 1997. SwArr.

[60] S. ITO, S. Tanaka, and H. Nakada. On unimodal transformations and chaos. Tokyo J. Math., 2:241-259, 1979. 
[61] R. Kapral and K. Showalter, editors. Chmical Waves and Patterns. Kluwer Acadamic Publishers, 1995.

[62] I. Katzorke. Modeling and analysis of production systems. PhD thesis, Universität Potsdam, 2002.

[63] I. Katzorke and A. Pikovsky. Chaos and complexity in a simple model of production dynamics. Discrete Dynamics in Nature and Society, 5:179-187, 2000.

[64] L. Kleinrock. Queuing Systems. John Wiley \& Sons, 1975.

[65] D.E. Knuth. The Art Of Computer Programming, Seminumerical Algorithms, volume 2. Addison-Wesley, Reading MA., 1981.

[66] C. Köhrmann. Modellbasierte Verfügbarkeitsanalyse automatischer Montagelinien. PhD thesis, University Hannover, Institute for Production Systems, 2000.

[67] S.R.T. Kumara, H. van Brussel, S.T.S. Bukkapatam, and I. Ham. Non-linear dynamics and chaos in manufacturing - a critical analysis. In CIRP international Seminar on Manufacturing Systems, 2001.

[68] E.R. Larsen, J.W.D. Morecroft, and J.S. Thomson. Complex behaviour in a production distribution model. European Journal of Operational Research, 119:61-74, 1999.

[69] J.D.C. Little. A proof for the queueing formula $l=\lambda w$. Operations Research, 9:383-387, 1960.

[70] O. Lorch, J. Denk, J.F. Seara, M. Buss, F. Freyberger, and G. Schmidt. An emulation enviroment for a vision guided virtual walking machine. In Proc. of the First IEEE-RAS International Conference on Humanoid Robots HUMANOID S2000, Cambridge, MA, 2000.

[71] J. Lunze. Modelling, Analysis and Design of Hybrid Systems, volume 279 of LNCIS, chapter What is a Hybrid System, pages 3-14. Springer Verlag ,Berlin, Heidelberg, 2002.

[72] K.J. Luo, H.T. Gran, K.H. Ploog, and L.L. Bonilla. Explosive bifurcation to chaos in weakly coupled semiconductor superlattices. Phys. Rev. Lett., 81(6):1290-1293, 1998.

[73] J Lygeros, K.H. Johansson, S.N. Simic, J. Zhang, and S. Sastry. Dynamical properties of hybrid automata. IEEE Trans. Autom. Control, 41(1):2-18, 2003.

[74] Y.L. Maistrenko, V.L. Maistrenko, and L.O. Chua. Cycles of chaotic intervals in a time -delayed chua's circuit. Int. J. Bifurcation Chaos Appl. Sci. Eng., 3(6):1557-1572, 1993.

[75] J. Mareczek, M. Buss, and G. Schmidt. Robust global stabilization of the underactuated 2-dof manipulator. Proc. of the IEEE International Conference on Robotics and Automation, Leuven, pages 2640-2645, 1998.

[76] A.S. Mikhailov. Foundations of Synergetics, volume 1. Springer, Berlin, 1994. 
[77] I. Mitrani. Simulation techniques for discrete event systems. Cambridge University Press, 1982.

[78] J. Müller and W. van Saarloos. Morphological instability and dynamics of fronts in bacterial growth models with nonlinear diffusion. Phys. Rev. E, 56:061111, 2002.

[79] H.E. Nusse and J.A. J.A. Yorke. Border-collision bifurcations for piecewise smooth one-dimensional maps. Int. J. Bifurcation Chaos Appl. Sci. Eng., 5(1):189-207, 1995.

[80] H.E. Nusse, E. Ott, and J.A. Yorke. Border collision bifurcations: An explanation for observed bifurcation phenomena. Phys. Rev. E, 49(2):1073-1076, 1994.

[81] H.E. Nusse and J.A. Yorke. Border collision bifurcations including "period two to period three". Physica D, 57:39-57, 1992.

[82] P. Nyhuis and H.-P. Wiendahl. Logistische Kennlinien. Springer Berlin Heidelberg New York, 1999.

[83] P. Orella and F. Claro. A terahertz molecular switch. Phys. Rev. Lett., 90(17):178302, 2003.

[84] S. Parui and S. Banerjee. Border collision bifurcations at the change of state space dimension. Chaos, 12(4):1054-1069, 2002.

[85] M. Patra, G. Schwarz, and E. Schöll. ifurcation analysis of stationary and oscillating domains in semiconductor superlattices with doping fluctuations. Phys. Rev. B, 57(3):1824-1833, 1998 .

[86] P.J. Ramadge and W.M. Wonham. The control of discrete event systems. Proc. IEEE, 77(1):81-98, 1989.

[87] B Rem and D. Armbruster. Control and synchronization in switched arrival systems. Chaos, 2003. to be published.

[88] N.F. Rulkov. Modeling of spiking-bursting neural behavior using twodimensional map. Phys. Rev. E, 65:041922, 2002).

[89] J.P.M. Schmitz, D.A. van Beek, and J.E. Rooda. Chaos in discrete production systems? Journal of Manufacturing Systems, 21(3):236-246, 2002.

[90] E. Schöll. Nonlinear spatio-temporal dynaics and chaos in semiconductors, volume 10 of Nonlinear Sciences Series. Cambridge University Press, Cambridge, 2001.

[91] B. Scholz-Reiter, M. Freitag, A. Schmiederer, and S. Müller. Nichtlineare dynamik und pps. PPS Management, 6(3):34-37, 2001.

[92] T. Schürmann and I. Hoffmann. The entropy of strange billiards inside nsimplexes. J. Phys. A: Math. Gen., 28:5033-5039, 1995.

[93] J.A. Sherratt. Invading wave fronts and their oscillatory wakes are linked by a modulated travelling phase. Physica D, 117:145-166, 1997. 
[94] S.N. Simic, K.H. Johansson, S. Sastry, and J. Lygeros. Towards a geometric theory of hybrid systems. In Hybrid Systems: Computation and Control, volume 1790 of LNCS, pages 421-436. Springer Verl., 2000.

[95] R. Stoop and C. Wagner. Scaling properties of simple limiter control. PRE, 90(15):154101, 2003.

[96] M. Timme, F. Wolf, and T. Geisel. Prevalence of unstable attractors in networks of pulse-coupled oscillators. PRL, 89(15):154105, 2002.

[97] J.E. Tolsma and P.I. Barton. Hidden discontinuities and parametric sensitivity calculations. SIAM J. Sci. Comput., 23(6):1861-1874, 2002.

[98] P. Vakili and G.-X. Yu. Periodic and chaotic dynamics of a switched-server system under corridor policies. IEEE Transactions of Automatic Control, 41(4):584-588, 1996.

[99] G. Wackenhut, V. Avrutin, and M Schanz. On dynamical systems with piecewise defined system functions. In Proceedings of the 2nd International Conference ”Tools for Mathematical Modelling” MATHTOOLS'99, pages 4-20, 1999.

[100] A. Wacker. Semiconductor superlattices: a model system for nonlinear transport. Phys. Rep., 357:1-111, 2002.

[101] C. Wagner and R. Stoop. Renormalization approach to optimal limiter control in 1-d chaotic systems. J. Stat. Phys., 106(1/2):97-107, 2002.

[102] D. Walgraef. Spatio-temporal Pattern Formation. Partially Ordered Systems. Springer Verl., Berlin, Heidelberg, New York, 1997.

[103] J. Wang, X.-L. Ding, B. Hu, B.-H. Wang, J.-S. Mao, and D.-R. He. Characteristics of a piecewise smooth area-preserving map. Phys. Rev. E, 64:026202, 2001.

[104] E. Westkämpfer, H.-H. Wiendahl, G. Pritscow, B. Rempp, and M. Schanz. Turbulenz in der pps - eine analogie. Werkstatttechnik, 90(5):203-207, 2000.

[105] H.-P Wiendahl, J. Worbs, and K. Peters. Turbulente zeiten. ZWF, 97(12):633637, 2002.

[106] J. Worbs and H.-P. Wiendahl. private communication.

[107] J. Worbs, H.P. Wiendahl, and K. Peters. Analysis of the logistical performance of manufacturing processes with methods of non-linear dynamics. In R. Teti, editor, Proc. 3rd CIRP (Intelligent Computation in Manufacturing Engineering) International Seminar, 2002.

[108] H. Ye, A.N. Michel, and L. Hou. Stability theory for hybrid dynamical systems. IEEE Trans. Autom. Control, 43(4):461-474, 1998.

[109] E. Yellin and A. Rabinovich. Properties and features of asymmetric partial devil's staircases deduced from piecewise linear maps. Phys. Rev. E, 67:016202, 2003. 



\section{Acknowledgements}

This thesis is the result of several years of work and influenced by a considerable number of people. All of them I would like to express my gratitude. The most important of them for this work I shall acknowledge in the following. First of all I would like to thank my advisor Prof. Parlitz for his constant support. He made this thesis possible in many ways.

The project that he has started with the Institute for Production Systems in Hannover gave me the chance to learn much about the problems of production engineering. I have to thank our partners, Prof. Wiendahl and Jochen Worbs for many fruitful discussions.

When I started the work on the hybrid switching systems considered here, it was not predictable that an application to supperlattices is possible. So my special thanks are due to Andreas Amann, with whom the ideas of the model for front dynamics were discussed many times. For the possibility to use the calculations of the microskopic model here I have to thank J.H. Schlesner. Prof. Schöll accompanied the work on this model with his constant interest. The financial support of the VW-Foundation (Grant No. I/76 279 - 280) is gratefully acknowledged.

Furthermore I would like to thank Prof. Lauterborn for the perfect working conditions in his institute. My roomates and colleagues Jochen Bröcker, Alexander Hornstein and Immo Wedekind supported me in many ways. Last but not least I thank B. Väth for her patience and support during the hard times when this thesis where written. 



\section{Lebenslauf}

\section{Persönliche Daten}

$\begin{array}{ll}\text { Name } & \text { Karsten Peters } \\ \text { Geburtsdatum } & \text { 30. November } 1972 \\ \text { Geburtsort } & \text { Rostock } \\ \text { Nationalität } & \text { deutsch }\end{array}$

\section{Ausbildung}

1979-1987 Besuch der POS in Rostock

1987 -1991 Besuch der Spezialschule "Albert Einstein" in Rostock

Juli $1991 \quad$ Abitur

1991-1992 Grundwehrdienst

1992-2003 Studium der Physik an der Universität Göttingen

Juli $1994 \quad$ Vordiplom

1998-2000 Diplomarbeit am Dritten Physikalischen Institut der Universität Göttingen, Betreuer: Dr. U.Parlitz

Titel: Untersuchungen zur Dynamik gekoppelter chaotischer Systeme

Mai 2000 Diplom

seit Juni 2000 wissenschaftlicher Mitarbeiter am Dritten Physikalischen Institut 